\title{
Entwicklung eines selbstschneidenden Häckslerkonzeptes zur vollmechanisierten Entbuschung von Offenflächen
}

\author{
Dissertation \\ zur Erlangung des Doktorgrades \\ der Fakultät für Agrarwissenschaften \\ der Georg- August- Universität Göttingen
}

\author{
vorgelegt von \\ Tim Wegener \\ geboren in Tulln/ Österreich
}

Göttingen, Juli 2011 
D 7

Referent: Dr. J.-K. Wegener

Korreferent: Prof. Dr. H. Van den Weghe

Tag der mündlichen Prüfung: 21.Juli 2011 


\section{Inhaltsverzeichnis}

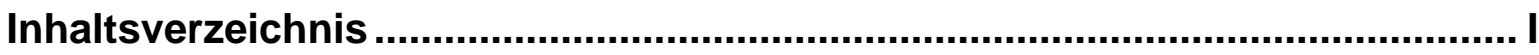

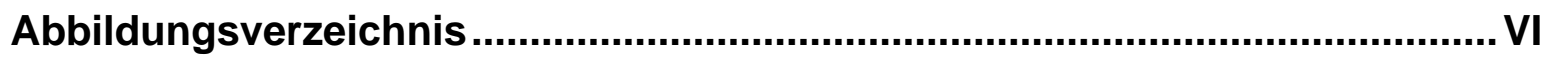

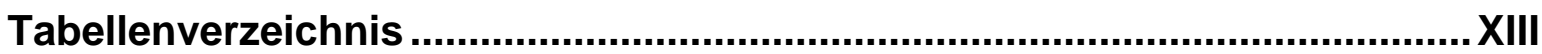

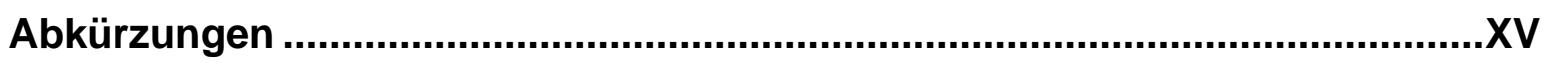

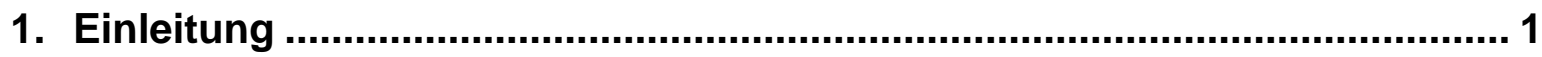

2. Hypothese und Zielstellung ..................................................................... 3

2.1. Das Projekt „Vollmechanisierte Landschaftspflege in

Naturschutz- und FFH- Gebieten“.................................................... 3

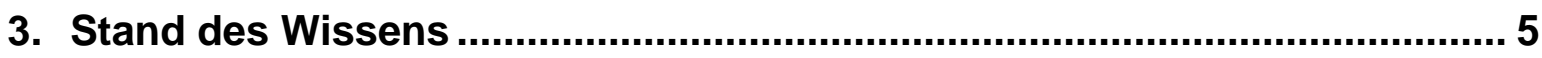

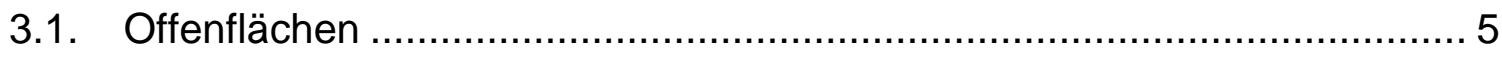

3.1.1. Definition der Offenflächenentbuschung ……................................ 6

3.1.2. Verbuschung von Offenflächen .................................................. 6

3.1.2.1. Verbuschungen in Deutschland .............................................. 9

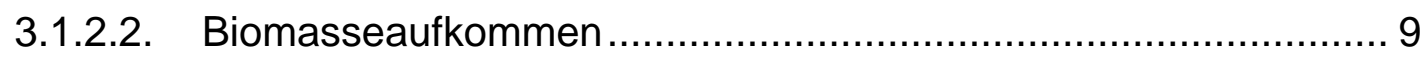

3.1.3. Ziele und Restriktionen bei der Entbuschung von Offenflächenbiotopen ............................................................... 10

3.2. Pflegeverfahren und Techniken zur Entbuschung ............................... 13

3.2.1. Unmechanisierte Verfahren und Techniken ................................... 13

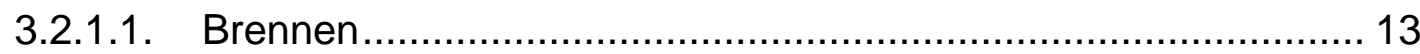

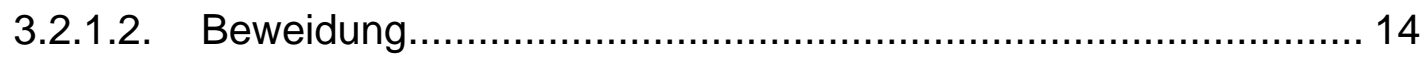

3.2.2. Teilmechanisierte bis vollmechanisierte Verfahren und

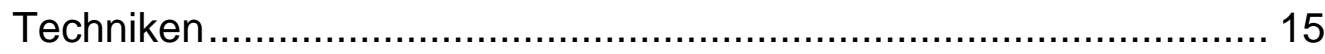

3.2.2.1. Manuelle/ Motormanuelle Entbuschung …................................ 15

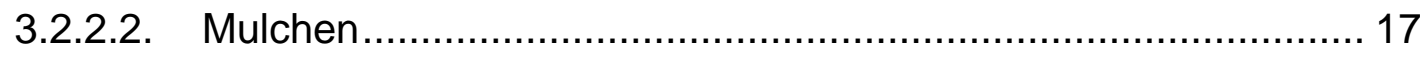

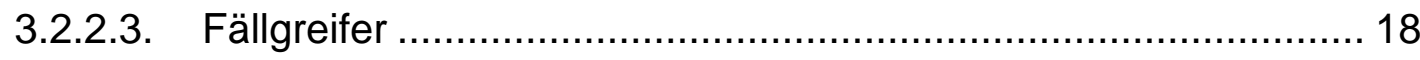




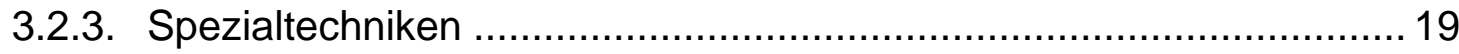

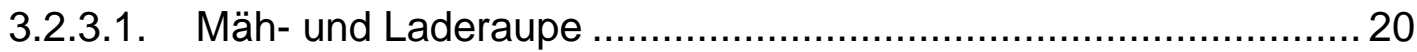

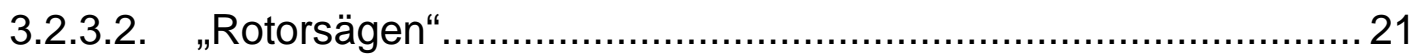

3.2.3.3. Forstmulcher mit Überladefunktion ......................................... 22

3.2.4. Räumung und Bergung des Entbuschungsmaterials......................... 23

3.3. Technikprinzipien der Holzzerkleinerung ………............................. 26

3.4. Qualitätsparameter von Holzhackschnitzeln ........................................ 30

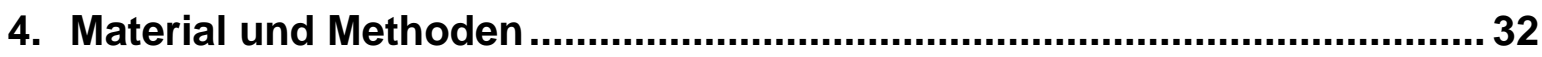

4.1. Vorgehensweise in der Entwicklung …….......................................... 32

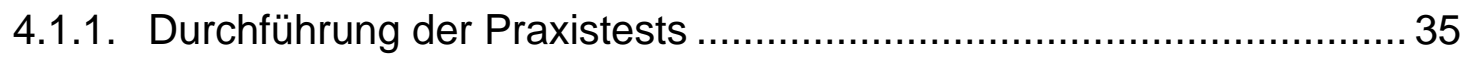

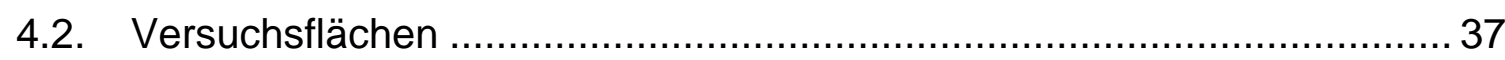

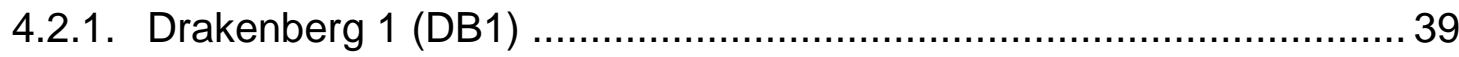

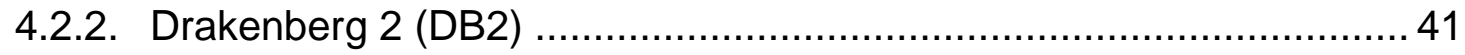

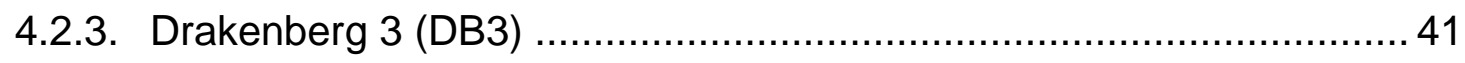

4.2.4. Ausgleichsfläche Roringen (AFR) ............................................ 42

4.3. Häckslerkonzept „Tritucap 2“ ............................................................ 43

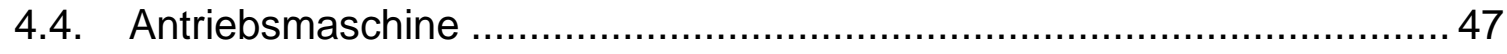

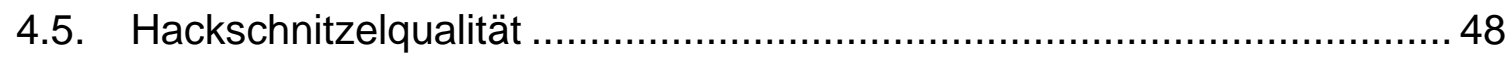

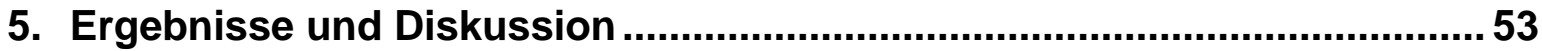

5.1. Eingrenzung des Verfahrens-/ Zielbereiches ........................................ 53

5.2. Anforderungsliste an ein vollmechanisiertes Verfahren zur flächigen Entbuschung von Offenflächen............................................. 59

5.2.1. Naturschutzfachliche Anforderungen ........................................... 59

5.2.2. Technische und technologische Anforderungen ................................ 61

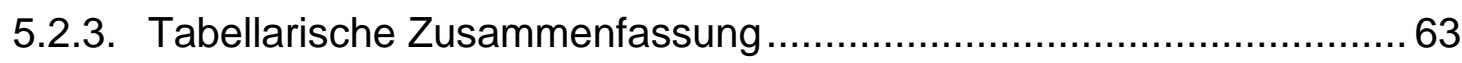

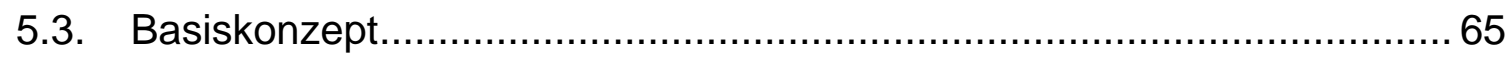


5.3.1. Potenzielle Eignung des selbstschneidenden Häckslerkonzeptes „Tritucap 2“ zur Entbuschung von

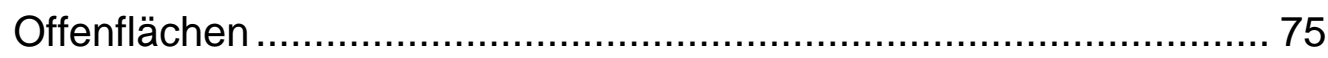

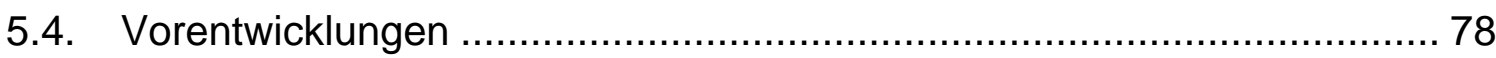

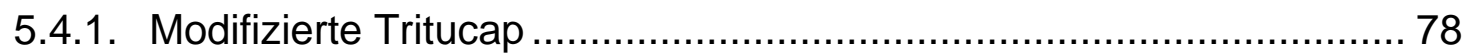

5.4.1.1. Umsetzung des Maschinenkonzeptes Tritucap ......................... 78

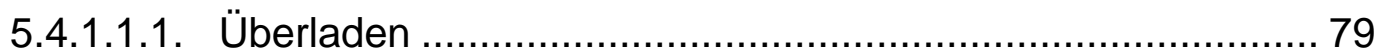

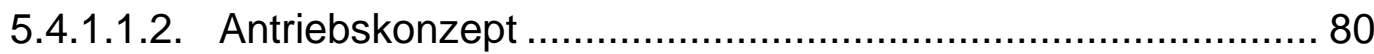

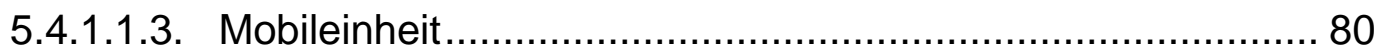

5.4.1.2. Praxiseinsätze der Tritucap .................................................. 81

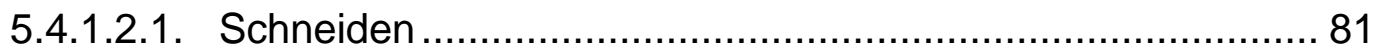

5.4.1.2.2. Aufnehmen, Zuführen und Zerkleinern .............................. 82

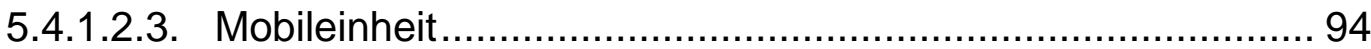

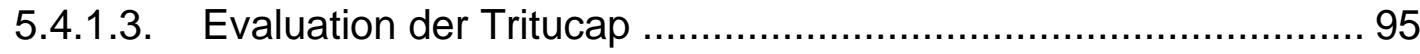

5.4.1.4. Zwischenfazit zur modifizierten Tritucap ................................. 97

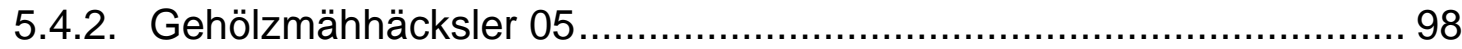

5.4.2.1. Konzeptentwicklung zum GMH $05 \ldots \ldots \ldots \ldots \ldots \ldots \ldots \ldots \ldots \ldots \ldots \ldots \ldots \ldots . . . . . . . . \ldots 8$

5.4.2.2. Umsetzung des Maschinenkonzeptes GMH 05...................... 102

5.4.2.2.1. Schneiden .................................................................. 103

5.4.2.2.2. Aufnehmen und Zuführen ............................................. 105

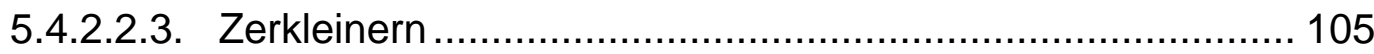

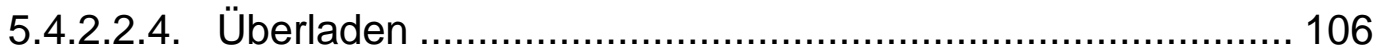

5.4.2.2.5. Antriebskonzept .......................................................... 107

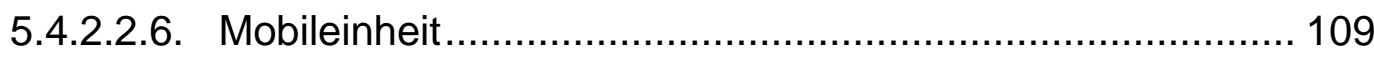

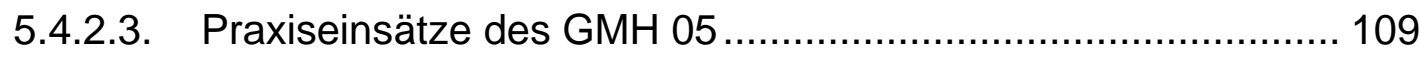

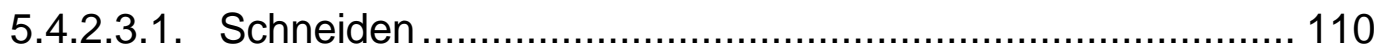


5.4.2.3.2. Aufnehmen und Zuführen............................................. 114

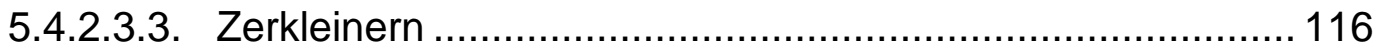

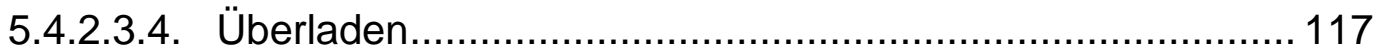

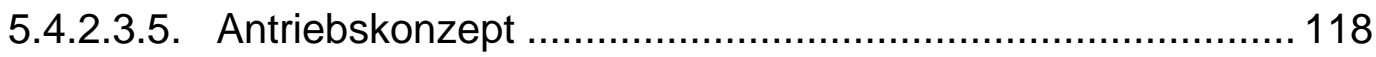

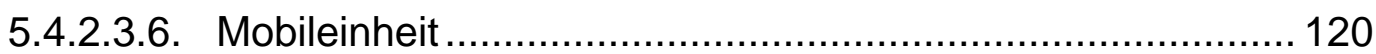

5.4.2.3.7. Hackschnitzelqualität ..................................................... 121

5.4.2.4. Evaluation des GMH 05 .................................................... 123

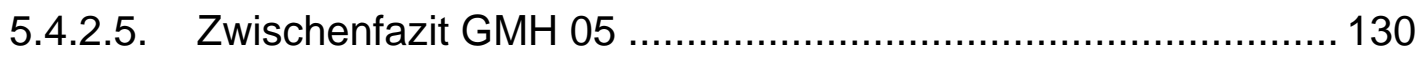

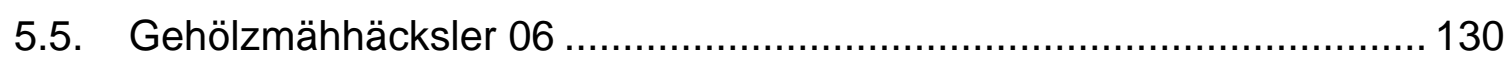

5.5.1. Konzeptentwicklung zum GMH 06........................................... 130

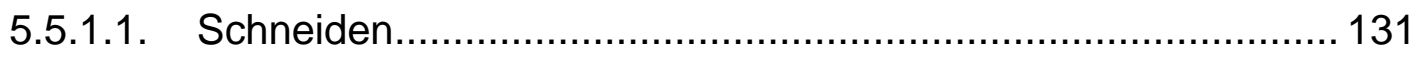

5.5.1.2. Aufnehmen und Fördern..................................................... 131

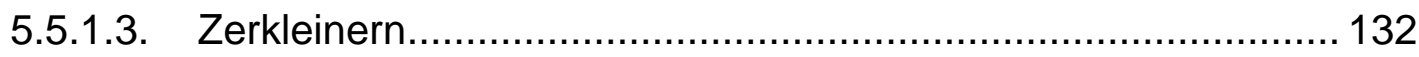

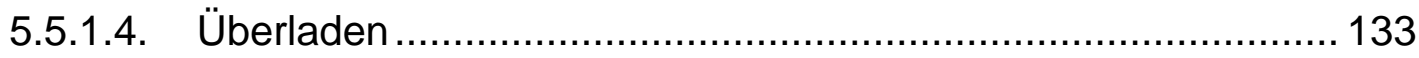

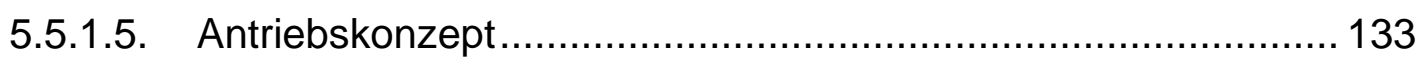

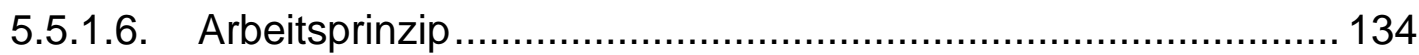

5.5.2. Umsetzung des Maschinenkonzeptes GMH 06........................... 135

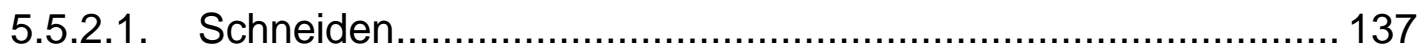

5.5.2.2. Aufnehmen und Zuführen ................................................... 138

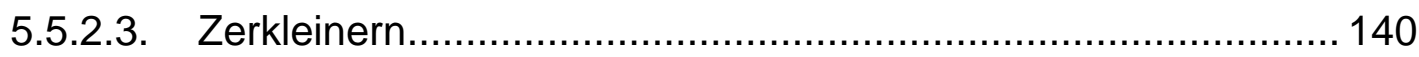

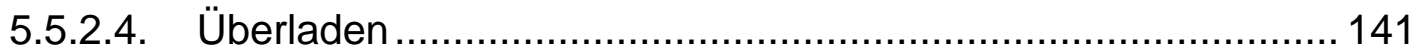

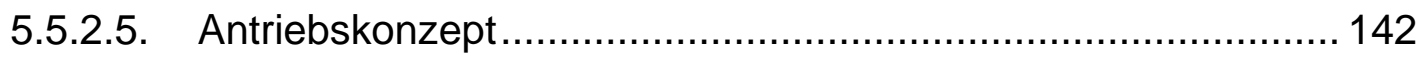

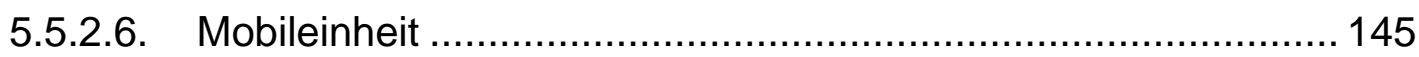

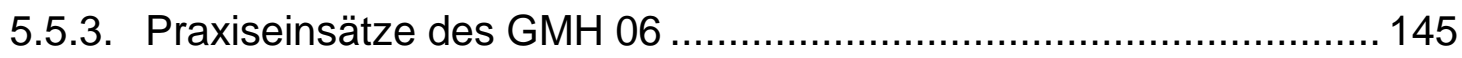

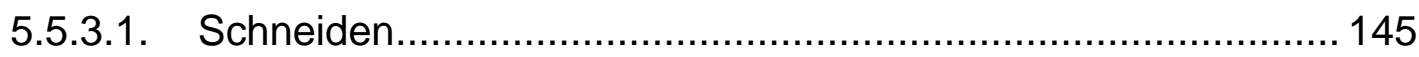

5.5.3.2. Aufnehmen und Fördern....................................................... 148 


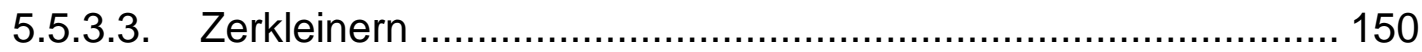

5.5.3.4. Überladen............................................................... 150

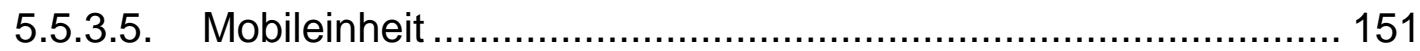

5.5.3.6. Hackschnitzelqualität.......................................... 151

5.5.4. Evaluation des GMH 06................................................... 157

5.6. Einordnung des Maschinenkonzeptes $\mathrm{GMHO6}$ in die Verfahren zur vollmechanisierten Beseitigung von Verbuschungen auf Offenflächen ........................................... 164

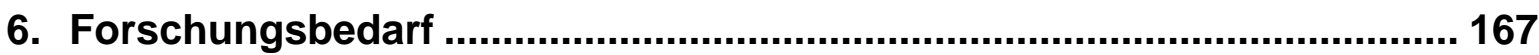

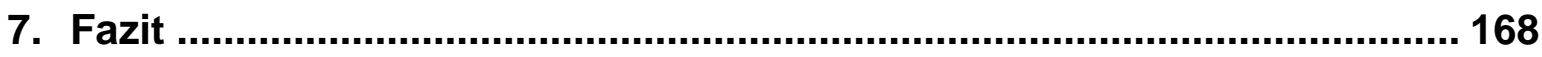

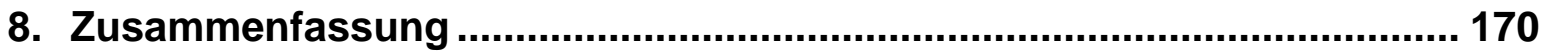

Literaturverzeichnis .................................................................................... 171

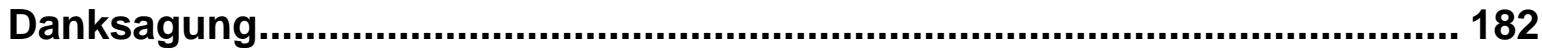

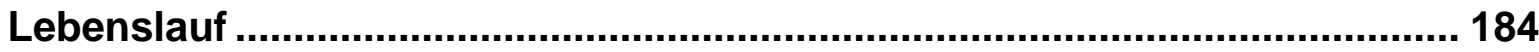




\section{Abbildungsverzeichnis}

Abb. 1: Schematische Darstellung der Gehölzsukzession über die Zeit (mit angedeuteter Störung des Prozesses) (GEYER \& DoLEK, 2011) ................ 7

Abb. 2: Wirkdreieck der Verknüpfung von Pflegeaufgabe, Standort und Pflegefläche bei der Auswahl der Art des Pflegeverfahrens (nach KROMER \& RELOE (1991), verändert)

Abb. 3: Mäh- und Laderaupe des Herstellers „mera Rabeler“; Im Besitz des Landschaftspflegeunternehmens Meyer-Luhdorf, Winsen/ Luhe (MEYER-LUdORF, 2009)

Abb. 4: Kompaktlader beim Fällen von Büschen in Namibia (links) mit robust ausgeführter Rotorsäge (rechts) (LEINONEN, 2007).

Abb. 5: Gängige Technikprinzipien zur Zerkleinerung von Holz (BRUSCHE, 1983, verändert) 26

Abb. 6: Entstehung von Hackschnitzeln im Trommelhacker (MAIER, 1983)

Abb. 7: Einzugsprinzip des Schneckenhackers, links: schematisch nach BRUSCHE (1983); rechts: Spanbildung bei der Zerkleinerung

Abb. 8: Bezeichnung von Komponenten und Geometrien am Schneckenhacker; B) dreigängiger Rotor (Quelle: Wegener, J.-K., Göttingen)

Abb. 9: Bezeichnungen von Komponenten und Geometrien am dreigängigen (rot, blau, gelb) Schneckenhacker und der Schneckenschneide (Quelle: Wegener, J.- K., Göttingen) 29

Abb. 10: Ablaufschema des Entwicklungsprozesses zur Konzeptentwicklung der vollmechanisierten Landschaftspflege 33

Abb. 11: Lage der Versuchsflächen im FFH- Gebiet „Göttinger Wald“; gelb = Ausgleichsfläche Roringen (AFR); rot umrandet = Kartenausschnitt Abb. 12 
Abb. 12: Karten- und Luftbildausschnitt zur Lage der Versuchsflächen auf dem Drakenberg; rot = Drakenberg 1 (DB1); grün = Drakenberg 2 (DB2); blau = Drakenberg 3 (DB3); Maßstab: 1:13000 (MEINEKE, 2006; verändert).

Abb. 13: Lage der Versuchsfläche „Ausgleichsfläche Roringen“ (AFR, gelb) im Südosten von Roringen bei Göttingen; Maßstab 1:10000; (Quelle: GoOgLEMAPS, 2011) 38

Abb. 14: Stark entwickelter Weißdornbusch (sehr dick, freistehend) auf der Versuchsfläche Drakenberg1 (DB1); Verzweigung des Stocks direkt über der Bodenoberfläche zu 11 Trieben (Zweimeter- Maß im Vordergrund liegend)

Abb. 15: Mähhäcksler „Tritucap 2“ am Einsatzort in Amazonien (BLock, 2004)

Abb. 16: Aufbau der Tritucap in der Grundform ohne

Überladeeinrichtung (skizziert). 46

Abb. 17: Schematische Darstellung des Siebvorganges (CEN/TS

15149-1, 2005); 1: Materialzuführung; 2: Zunehmende Lochdurchmesser; 3: Richtung des Materialflusses 52

Abb. 18: Technisch bearbeitbare Wurzelhalsdurchmesser von verschiedenen Anwendungsverfahren zur Entbuschung von Offenflächen (Betrachtungsbereich bis $30 \mathrm{~cm}$ ) 54

Abb. 19: Bearbeitbare Wurzelhalsdurchmesser von verschiedenen Anwendungsverfahren zur Entbuschung von Offenflächen (Betrachtungsbereich bis $30 \mathrm{~cm}$ ); grau/weiß : Verfahrensbereich mit Anwendungseinschränkung; rot schraffiert: Bereich eingeschränkter Technikverfügbarkeit(ca. 3- $5 \mathrm{~cm}$ ) .57 
Abb. 20: Bearbeitbare Wurzelhalsdurchmesser von verschiedenen Anwendungsverfahren zur Entbuschung von Offenflächen; Betrachtungsbereich bis $30 \mathrm{~cm}$; grau/weiß: Verfahrensbereich mit Anwendungseinschränkung; rot schraffiert: Bereich eingeschränkter Technikverfügbarkeit (ca. 3- $5 \mathrm{~cm}$ ); grüner Bereich: Anwendungsbereich des zu entwickelnden Maschinenkonzeptes (ca. $<1-10 \mathrm{~cm}$ und darüber) 58

Abb. 21: Transportvolumen der gleichen Menge Holz in Abhängigkeit von der Beschaffenheit (Quelle: Timperi; A.; Finnland) 68

Abb. 22: MULAG Heckenschneidkopf HSK 1200 bei der AutobahnMittelstreifenpflege mit gleichzeitiger Absaugung des Häckselmaterials (MULAG FAHRZEUGWERK HEINZ WöSSNER, 2006)

Abb. 23: Selbstschneidendes Häckslersystem „Tritucap 2“ bei der Flächenbereitung im Rahmen der nichtbrennenden Mulchwirtschaft in Amazonien (Quelle: Block) 76

Abb. 24: Verteilung der Hackschnitzelqualitäten auf die definierten Größenklassen, Bearbeitungsmaschine: Tritucap 2; Bezugsgröße: Masse der Gesamtprobe (BLOCK, 2004)

Abb. 25: Modifizierte Tritucap mit Überladeeinrichtung und Frontladercontainer bei vollmechanisierter Entbuschung 80

Abb. 26: Spitzbügel an Tritucap mit Überladeeinrichtung

Abb. 27: Ideal geklemmter Trieb im Häckselspalt der Tritucap bei der Zerkleinerung; 1. tangentialer Transport zum Häckselspalt; 2. rotoraxialer Einzug zur Zerkleinerung; 3. Einzug der Triebachse annähernd parallel zum Pfeil bei waagerechtem Einzug der Zerkleinerung 83

Abb. 28: Beobachtete Tendenz zum waagerechten Einzug der Triebe beim Maschinenkonzept der Tritucap in Abhängigkeit von Vegetationsbedingungen (Breite entspricht Größe der Tendenz) 85 
Abb. 29: Typische Wuchsformen von Gehölzen; links: buschartig; rechts: baumartig

Abb. 30: Verstopfung des Häckselspaltes durch verstärkt waagerecht eingezogenes Material dünneren Durchmessers

Abb. 31: Geöffnete Wartungsklappe am ersten Beschleuniger der

Tritucap nach Blockade durch dünnes und überlanges

Gehölzmaterial nach waagerechtem Triebeinzug durch die Häckselrotoren

Abb. 32: Rechter Häckselrotor der modifizierten Tritucap 3 mit insgesamt acht Auswerfern pro Häckselrotor

Abb. 33: Linker Häckselrotor der modifizierten Tritucap 3 mit

Auswerfermantel

Abb. 34: Umbau eines Häckselrotors an der Tritucap: Verlängerung der

Schneidwindungen und Integration von drei Auswerfern je

Schneidwindung ohne Überstand über die Schneide; Anpassung

der Seitenwand und des Häckselspaltes.

Abb. 35: Auf Seitenwand der Tritucap installierte Förderschnecke

(hydrostatisch angetrieben)

Abb. 36: Einseitig montierter Förderstern auf Tritucap zur Unterstützung der Zuführung der Triebe zum Häckselspalt.

Abb. 37: Blockade des Häckselrotors mit verlängerten Windungen

durch den gleichzeitigen Einzug einer mehrtriebigen Hundsrose

(Blick von oben in den Häckselspalt).

Abb. 38: Feldhäcksler mit Erntevorsatz für Kurzumtriebsplantagen

(Quelle: Wippermann, Hamburg)

Abb. 39: Konstruktionsprinzip des Gehölzmähhäckslers 05 (GMH05);

Richtung der Blockpfeile entspricht der Drehrichtung................................102

Abb. 40: Gehölzmähhäcksler 05 im Entbuschungseinsatz ................................103

Abb. 41: Sägeblattbalken des Gehölzmähhäckslers 05 mit

Förderzylindern auf jeweils äußerem Sägeblatt 
Abb. 42: Blick in die rechte Häckseleinheit des Gehölzmähhäckslers 05 zwischen dem Zuführwalzenpaar hindurch 106

Abb. 43: Antriebskonzept des Gehölzmähhäckslers 05 (schematisch) 107

Abb. 44: Ansicht des Gesamtmaschinensystems zur vollmechanisierten Entbuschung mit dem Gehölzmähhäcksler 05 109

Abb. 45: Schnittfläche eines mit dem Sägeblattbalken des Gehölzmähhäckslers 05 durchtrennten Weidentriebs 110

Abb. 46: Hohe Bestandesdichte und vermehrt dickere Weißdornbüsche behindern waagerechte Ausrichtung der Gehölze mit dem Gehölzmähhäcksler 05

Abb. 47: Gehölzmähhäcksler 05 bei der Mähernte im dicht mit Eschen verbuschten Bereich auf der Ausgleichsfläche in Roringen

Abb. 48: Diagonal bzw. quer zur Zuführrichtung liegende Gehölztriebe als Störung vor den Aufnahmeaggregaten des Gehölzmähhäckslers 05

Abb. 49: Durch zu tiefe Förderung der Zuführwalzen hervorgerufene Auftrennung von Trieben längs zur Triebachse..... 115

Abb. 50: Mit gezahnten Förderelementen ausgestatteter Förderzylinder des Gehölzmähhäckslers 05

Abb. 51: Schäden im Antriebsstrang des Gehölzmähhäckslers 05 durch schädliche Lastwechsel und Lastspitzen

Abb. 52: Ergebnisse der Siebanalyse nach CEN/TS 15149-1 einer Hackschnitzelmischprobe produziert mit dem Gehölzmähhäcksler 05

Abb. 53: Blick in den Zuführtrichter eines manuell beschickbaren Schneckenhäckslers bei der Zerkleinerung; weißer Pfeil: Drehrichtung der Häckselschnecke; roter Pfeil: behinderte Zuführrichtung durch Gehölztriebe während der Verarbeitung; schwarzer Pfeil: weitere Zuführung möglich 133 
Abb. 54: Arbeitsprinzip des Gehölzmähhäckslers 06 bei der vollmechanisierten Entbuschung.... 135

Abb. 55: Gehölzmähhäcksler 06 im Frühjahr 2007 nach der Fertigstellung

Abb. 56: Konstruktionszeichnung des Gehölzmähhäckslers 06 (vereinfachte Darstellung mit einer Förderschnecke, ohne Förderwalze, Auswurfturm und Drückbügel)

Abb. 57: Blick in den Arbeitsbereich des Gehölzmähhäckslers 06 mit Zuführschnecken (rot), Scharnierbandboden (blau) und Förderzylindern (silber) 138

Abb. 58: Blick auf die Schneid- und Zuführkomponenten des Gehölzmähhäckslers 06. Über dem Übergang von Sägeblattbalken und Scharnierbandboden (blau) ist eine hydraulisch angetriebene Förderwalze installiert. 139

Abb. 59: Blick in den Häckselspalt des Gehölzmähhäckslers 06 141

Abb. 60: Hydraulische Eigenversorgung des Gehölzmähhäckslers 06 mit a) Keilriemenantrieb; b) Dreifachpumpe; c) Öltank (100 Liter) mit Ölfiltern; d) Ölmotor des Sägeblattantriebs 143

Abb. 61: Antriebskonzept des Gehölzmähhäckslers 06 (skizziert, dabei sind Ölkreisläufe nur angedeutet und nicht detailgetreu dargestellt); mit:

Abb. 62: Gehölzmähhäcksler 06 bei Ersteinsätzen in Birkensukzession am Waldrand 146

Abb. 63: Abbruch der vollmechanisierten Entbuschung mit dem

Gehölzmähhäcksler 06 durch eine zu hohe Bestandesdichte in buschartiger Vegetation mittleren Wurzelhalsdurchmessers $(6-8 \mathrm{~cm}$ WHD) 148

Abb. 64: Gehölzmähhäcksler 06 nach Austausch der Förderschnecken gegen glatte Seitenwände zur Materialkonzentration zum Häckselaggregat 
Abb. 65: In den Einsatzversuchen mit dem Gehölzmähhäckslers 06 produzierte Hackschnitzel

Abb. 66: Ergebnisse der Häufigkeit der Hackgutgrößen (kumuliert) aus einer Mischprobe von Entbuschungsmaterial; Material hergestellt bei Versuchseinsätzen mit Gehölzmähhäcksler 06 (Siebanalyse nach CEN/TS 15149-1)

Abb. 67: Häufigkeit von Hackgutgrößen (dargestellt in Massenprozent kumuliert) in Hackgutproben, hergestellt mit dem Gehölzmähhäcksler 06 bei manueller Beschickung zur Zerkleinerung; eingetragen sind die obere und untere Anteilsgrenze (kumuliert) von Hackschnitzelgrößenklassierungen der gängigen Norm CEN/TS 14691 (Siebanalyse nach CEN/TS 15149-1) 155

Abb. 68: Häufigkeit von Hackgutgrößen (dargestellt in Massenprozent kumuliert) in Hackgutproben verschiedener Sortierungsmischungen, hergestellt mit dem Gehölzmähhäcksler 06 bei manueller Beschickung bei der Zerkleinerung; eingetragen sind die obere und untere Anteilsgrenze (kumuliert) von Hackschnitzelgrößenklassierungen der gängigen Norm CEN/TS 14691 (Siebanalyse nach CEN/TS 15149-1) 156

Abb. 69: Tendenzen der Änderung des Entbuschungsvermögens des GMH 06 bei der Entbuschung von Offenflächen in Abhängigkeit von der Ausprägung von Vegetationsparametern; Pfeilbreite gleich Höhe des Entbuschungsvermögens 158

Abb. 70: Blick auf die Versuchsfläche Drakenberg 2 (DB2) vor (links, spätes Frühjahr 2007) und nach der Entbuschung mit dem Gehölzmähhäcksler 06 (Winter 2007) 


\section{Tabellenverzeichnis}

Tab. 1: Einteilungsklassen der Verbuschungen nach Stammform, Wurzelhalsdurchmesser, Bestandesdichte und Bestandesbreite mit ihren Ausprägungen 36

Tab. 2: Zusammenfassende Darstellung der potenziellen Erfüllbarkeit ausgewählter Ziele bestehender Entbuschungsverfahren der Offenflächenpflege sowie deren einschränkende Verfahrenseigenschaften + : gegeben; - : nicht gegeben; + - : je Verfahrensausführung bzw. Verbuschungsstruktur; 55

Tab. 3: Naturschutzfachliche Anforderungen zur Entwicklung eines vollmechanisiertes Häckslerkonzeptes zur Entbuschung von Offenflächen

Tab. 4: Technische und technologische Anforderungen zur Entwicklung eines vollmechanisiertes Häckslerkonzeptes zur Entbuschung von Offenflächen 65

Tab. 5: Einordnung der Siebanalyse einer Mischprobe des Häckselmaterials des Gehölzmähhäckslers 05 nach CEN/ TS 15149; Kastenlänge unter Größenbereichen entspricht deren Summe; gestrichelter Kasten entspricht der Einordnung; n.a. $=$ nicht aufgenommen. 122

Tab. 6: Erfüllung bzw. potenzielle Erfüllbarkeit naturschutzfachlicher Anforderungen nach Kenntnisstand der Vorentwicklung; $v$ : erfüllt; $x$ : nicht erfüllt (+/- : positive/negative Tendenz zur Erfüllbarkeit); ? : bisher nicht sicher abschätzbar 128

Tab. 7: Erfüllung bzw. potenzielle Erfüllbarkeit technischer und technologischer Anforderungen nach Kenntnisstand der Vorentwicklung; $v$ : erfüllt; $\mathrm{x}$ : nicht erfüllt (+/- : positive/negative Tendenz zur Erfüllbarkeit); ? : bisher nicht sicher abschätzbar 
Tab. 8: Siebanalyse der Hackschnitzel (nach CEN/TS 15149-1) einer manuell zugeführten Zerkleinerung bestimmter WurzelhalsDurchmesserklassen mit dem Gehölzmähhäcksler 06; dargestellt als Massenanteile der Siebfraktionen in Prozent; WD = Weißdorn 154

Tab. 9: Erfüllung bzw. potenzielle Erfüllbarkeit naturschutzfachlicher Anforderungen anhand der Ergebnisse zum GMH 06; v : erfüllt; x : nicht erfüllt (+/- : positive/negative Tendenz zur Erfüllbarkeit); ? : bisher nicht sicher abschätzbar

Tab. 10: Erfüllung bzw. potenzielle Erfüllbarkeit technischer und technologischer Anforderungen anhand der Ergebnisse zum GMH 06; v : erfüllt; $x$ : nicht erfüllt (+/- : positive/negative Tendenz zur Erfüllbarkeit); ? : bisher nicht sicher abschätzbar 


\section{Abkürzungen}

a

AFR

$\mathrm{BHD}$

BNatSchG

DB

FM

$\mathrm{GMH}$

ha

$\mathrm{kW}$

$\mathrm{t}$

TM

$\mathrm{U} / \mathrm{min}$

WHD
Jahr

Ausgleichsfläche Roringen

Brusthöhendurchmesser [cm]

Bundesnaturschutzgesetz

Drakenberg

Frischmasse [g; kg; t]

Gehölzmähhäcksler

Hektar $\left(=10.000 \mathrm{~m}^{2}\right)$

Einheit der Leistung in Kilowatt

metrische Tonne $(=1000 \mathrm{~kg})$

Trockenmasse [g; kg; t]

Einheit der Drehzahl in Umdrehungen pro Minute

Wurzelhalsdurchmesser [cm] 



\section{Einleitung}

Der Aufwuchs von Pioniergehölzen ist beim Erhalt und der Pflege von landschaftlich und biologisch wertvollen Offenflächenbiotopen eines der Kernprobleme in Mitteleuropa. Schützenswerte Offenflächenbiotope, wie Heiden und Kalkmagerrasen, und sogar ganze Landschaften, die durch das Offenland geprägt werden, können bei voranschreitender Sukzession durch die zunehmende Etablierung von Büschen und Bäumen in ihrer Existenz gefährdet oder gar vernichtet werden. Man spricht von der "Verbrachung“ oder treffender der „Verbuschung“. In der Regel führt die Zunahme von Gehölzen auf Offenlandbiotopen zu einer Abnahme der Biodiversität der dort speziell angepassten Flora und Fauna sowie der Verschiebung des für das Offenland typischen Artenspektrums. Vor allem die an trockene und lichtreiche Bedingungen angepassten Arten schwinden mit steigender Gehölzdichte und führen zu einer Degradation der Biotope (BöHMER, 1994; ROTTER, 2006).

Eine wichtige Maßnahme zur Pflege und zum Erhalt von schützenswerten Offenflächen sowie auch allein zur Bewirtschaftbarkeit in der extensiven Nutzung stellt die wiederkehrende Zurückdrängung und Beseitigung der aufstrebenden Pioniergehölze dar (RATHS ET AL., 1995; MüNZEL \& SCHUMACHER, 1993). Handelt es sich um das Entfernen von Gehölzen auf einer vorherigen Freifläche, spricht man von der „Entbuschung“ oder „Entkusselung“. Hierzu finden verschiedene Verfahren und Techniken Anwendung, welche je nach naturschutzfachlichem Ziel, Verfügbarkeit und ökonomischer sowie technischer Durchführbarkeit eingesetzt werden.

Nicht nur aus naturschutzfachlicher Sicht wird bei der Entbuschung eine Abfuhr des Entbuschungsmaterials von der Fläche gefordert. Seit einigen Jahren wird durch eine steigende Rohstoffnachfrage verstärkt die Nutzung des Gehölzmaterials von Entbuschungsmaßnahmen propagiert, um ein bisher nur wenig genutztes Dendromassepotenzial zu erschließen. Speziell bei der flächigen Entbuschung mit gleichzeitiger Materialabfuhr wird jedoch ein gewisses Defizit an verfügbarer Technik gesehen, um innerhalb naturschutzfachlicher Anforderungen Verbuschungen effizient von 
Offenflächen beseitigen zu können. Kern dieser Arbeit ist es ein entsprechendes Verfahren zu entwickeln und dieses in der Praxis einzusetzen. 


\section{Hypothese und Zielstellung}

Nach einer ersten Einschätzung der naturschutzfachlichen und technischen Anforderungen des Einsatzbereiches kann ein möglicher technischer Lösungsansatz zur vollmechanisierten Entbuschung von Offenflächen in der modifizierten Anwendung eines speziellen selbstschneidenden Häckslersystems vermutet werden. Dieses wird an der Abteilung Agrartechnik der Georg- August- Universität Göttingen im Rahmen eines vormals durchgeführten Projektes entwickelt. Es könnte zumindest als Vorlage zur Entwicklung eines neuen selbstschneidenden Häckslerkonzeptes dienen, um die Handlungsmöglichkeiten bei der Entbuschung von Offenflächen zu erweitern und die Basis für ein neues vollmechanisiertes Pflegeverfahren zu stellen.

Ziel dieser Arbeit ist es, durch die Entwicklung eines angepassten selbstschneidenden Häckslerkonzeptes eine technische Grundlage für ein Verfahren zur vollmechanisierten Entbuschung zu schaffen. Dieses soll ein Defizit an Technikverfügbarkeit zur Entbuschung von Offenflächen ausgleichen können. Dabei gilt es, die Funktion des Maschinenkonzeptes sicher zu stellen und in der Verfahrensentwicklung naturschutzfachliche Belange sowie auch eine gleichzeitige Materialbergung zu berücksichtigen. Das Konzept wird stufenweise in einem Entwicklungsprozess von einer Planungsphase über den Bau und den Einsatz von drei Prototypen entwickelt. Die Prototypen werden während der Entwicklung als Funktionsmuster in der praktischen Anwendung getestet.

\subsection{Das Projekt „Vollmechanisierte Landschaftspflege in Naturschutz- und FFH- Gebieten“}

Die Entwicklung des selbstschneidenden Häckslerkonzeptes zur Entbuschung von Offenflächen wird im Rahmen des interdisziplinären Verbundprojektes „Vollmechanisierte Landschaftspflege in Naturschutz- und FFH- Gebieten" durchgeführt, welches von der Deutschen Bundesstiftung Umwelt (DBU) finanziell gefördert wird (Projekt- Nummer 22629-34/0). Die vorliegende Arbeit stellt den technischen Teil des Forschungsprojektes dar, 
welcher von der Abteilung Agrartechnik der Georg-August- Universität Göttingen in Zusammenarbeit mit einem Maschinenbauunternehmen sowie einem land- und forstwirtschaftlichen Lohnunternehmer bearbeitet wird. Die naturschutzfachliche Begleitforschung wird wissenschaftlich von einem weiteren Hochschulinstitut sowie einem umweltbiologischem Ingenieurbüro begleitet. Fachlich sind zudem als genehmigende Stellen die für die Versuchsflächen zuständigen Behörden eingebunden. Das Projekt wird im Zeitraum von 2005 bis 2008 durchgeführt. 


\section{Stand des Wissens}

\subsection{Offenflächen}

Eine Offenfläche oder auch das Offenland stellt einen Raum dar, der nicht durch Gehölzvegetation geprägt ist. Eine sonnenliebende und meist speziell an die Bedingungen des Offenlandes angepasste Flora und Fauna dominiert die Artenzusammensetzung (BURKART, 2006). Neben natürlichen Offenflächen wie Mooren, Steppen oder Karste sind es vor allem die durch den Einfluss des Menschen entstandenen baum- und straucharmen Flächen, zu denen Heiden, Weiden und Wiesen aber auch die Ackerflächen zählen.

Im Laufe der Bewirtschaftung haben sich vor allem auf aus heutiger Sicht extensiv bearbeiteten Weideflächen spezielle Biotope herausgebildet, die erhaltens- und schützenswert sind. Die entstandenen Biotoptypen können entsprechend der ursprünglichen oder aktuellen Bewirtschaftungsform sowie auch der vorherrschenden Gelände- und Klimabedingungen sehr vielfältig sein. Als Beispiel soll an dieser Stelle nur der Magerrasen genannt werden, der nach VON DRACHENFELS (2004) vom Haupttyp in Untergruppen, wie z.B. Borstgras- Magerrasen, Sand- Magerrasen und Kalk- Magerrasen, eingestuft werden kann. Die Untergruppen lassen sich wiederum weiter auf den vorliegenden Biotoptyp spezifizieren. So kann der Kalk- Magerrasen in einer nächsten Stufe in Typischer Kalk-Magerrasen, Saumartenreicher KalkMagerrasen, Kalkmagerrasen-Pionierstadium und Blaugras-Kalkrasen eingeordnet werden.

Um den Fortbestand wertvoller Offenlandbiotope bzw. - landschaften zu sichern und einem weiteren Verlust begegnen zu können, sind eine Vielzahl dieser Offenflächen unter nationale sowie auch internationale Schutzbestimmungen gestellt worden. Hierzu zählen einerseits die einzelnen Naturschutzgesetze der Länder, aber auch z.B. die FFH- Richtlinie der EU (Flora- Fauna- Habitat- Richtlinie), die den unter Schutz gestellten Flächen einen gesetzlich gesicherten Pflege- und Erhaltungsanspruch garantieren. Entsprechende Managementpläne für die Flächen enthalten die notwendigen Erhaltungs- und, wenn notwenig, Wiederherstellungsmaßnahmen. Dazu 
zählen unter anderem auch Entbuschungsmaßnahmen, um den Offenflächencharakter einer Fläche wiederherzustellen.

\subsubsection{Definition der Offenflächenentbuschung}

Wie eingangs beschrieben wird die Beseitigung von Gehölzen auf vorherigen Freiflächen als „Entbuschen“, „Entkusseln“ oder „Freistellen“ bezeichnet. Dieser Vorgang ist jedoch nicht mit einem Kahlschlag, einer Heckenbeseitigung oder auch nicht mit dem sogenannten „Auf-den-Stocksetzen“ einer Heckenstruktur zu verwechseln. Vielmehr geht es um das Entfernen von Gehölzen aus der Strauchvegetation im Rahmen der natürlichen Gehölzsukzession, welche aus Zwergsträuchern, Sträuchern aber auch jungen Bäumen bestehen kann. Ihr Horizont im Bereich der Strauch- und Baumschicht bewegt sich im ungefähren Bereich von $60 \mathrm{~cm}$ bis $5 \mathrm{~m}$. Dabei werden die Gehölze nicht gerodet, sondern bodennah abgetrennt. Die Wurzeln und Triebstümpfe verbleiben auf der Fläche (JEDICKE, 1996; RAHMANN, 2000). Entbuschungsmaßnahmen werden zur Landschafts- und Biotoppflege, aber auch zum Erhalt der Flächenproduktivität, wie in der extensiven Grünlandnutzung, anhand verschiedener Verfahren und Techniken durchgeführt.

\subsubsection{Verbuschung von Offenflächen}

Bei dem Vorgang der Etablierung von Gehölzen auf einer gehölzarmen oder gehölzfreien Fläche spricht man allgemein von der „Verbuschung“. Sie ist Teil der natürlichen Vegetationsdynamik (der Sukzession), welche vereinfacht ausgedrückt, einen fortschreitenden Veränderungsprozess der Lebensgemeinschaften in einem Biotop darstellt, der durch veränderte Umweltbedingungen ausgelöst wird und einem neuen Gleichgewicht zustrebt (eine nähere Darstellung der unterschiedlichen Definitionsweisen ist z.B. in der Arbeit von FELINKS (2000) sehr umfassend dargestellt worden). Nach SCHREIBER (2000) führt z.B. die Nutzungsaufgabe auf potenziell waldfähigen

Standorten in Mitteleuropa über verschiedene Verbuschungs- und Vorwaldstufen zum Wald (Abb. 1). 


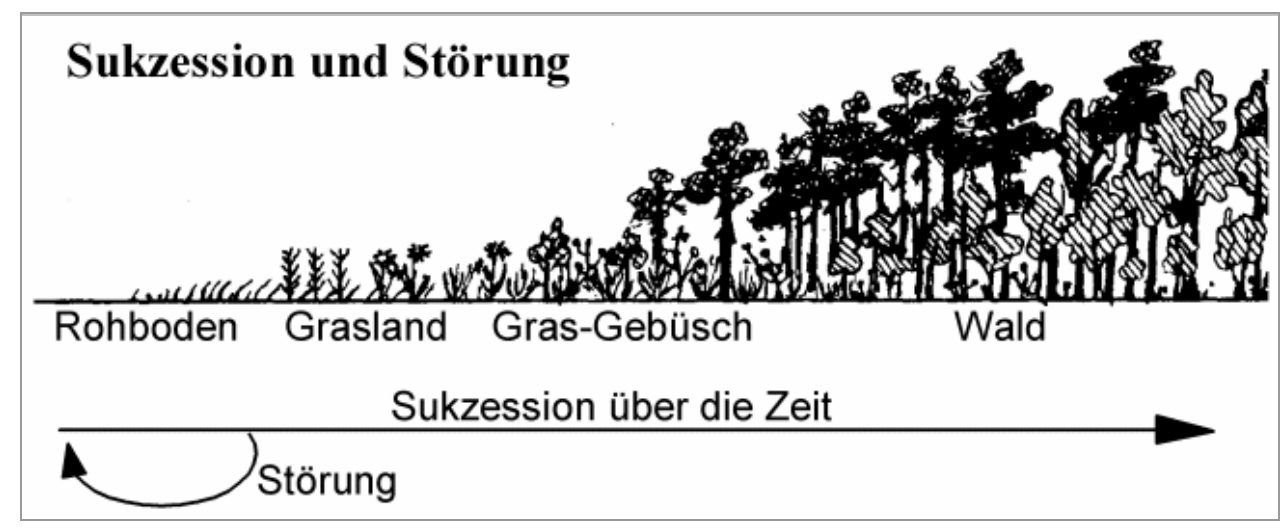

Abb. 1: Schematische Darstellung der Gehölzsukzession über die Zeit (mit angedeuteter Störung des Prozesses) (GEYER \& DOLEK, 2011)

Im Raum Göttingen sind es vor allem die Pionierbaumarten Esche (Fraxinus spec.), Birke (Betula spec.), Hartriegel (Cornus spec.), Weißdorn (Crataegus spec.), Schwarzdorn (Prunus spec.) und Rose (Rosa spec.), welche die Flächen erschließen (Dillenburger, 2005). Aber auch Pappel (Populus spec.), Weide (Sallix spec.), Robinie (Robinia spec.), Erle (Alnus spec.), Kiefer (Pinus spec.) und Hasel (Corylus spec.) werden beispielsweise immer wieder im Rahmen von Verbuschungen genannt. Dabei kann es sein, dass partiell oder über eine gesamte Fläche Arten dominieren, aber auch Mischungen in verschiedensten Ausprägungen möglich sind (PERINGER, 2008; MünZEL \& SCHUMACHER, 1993).

Die Ausbreitung der Gehölze über eine Offenfläche erfolgt oft von bestehenden Gehölzen vom Rand der Fläche oder von Gehölzinseln auf der Fläche aus. Diese sogenannten „Ammenpflanzen“ konnten sich etablieren und sorgen für eine generative oder auch vegetative Gehölzvermehrung. Besonders ist hier der Schwarzdorn (die Schlehe) hervorzuheben, welcher sich mittels seiner Samen, aber auch polykormon über Wurzelsprosse, verbreiten kann. Schwarzdorn zeigt deshalb ein typisches „Hineinwachsen“ in die Fläche von etablierten Ammenpflanzen aus, auch wenn sie vor der Samenreife geschnitten werden (BÖHMER, 1994; KoLLMANN \& STAUB, 1995).

Da die Sukzession im Hinblick auf die Gehölzentwicklung einen zeitlich und räumlich voranschreitenden Prozess darstellt, ist grundsätzlich bei Verbuschungen von sehr heterogen entwickelten Gehölzbeständen 
auszugehen. Das Alter und das Stadium der Gehölzsukzession geben hier die zu erwartende Größenentwicklungen der Gehölze vor. Dabei ist auf den erstbesiedelten Stellen die Altersstruktur am höchsten. Durch fortwährenden Neuaufwuchs liegen in der Regel Alters- und entsprechende Größenstrukturen der Gehölze vom Beginn der Verbuschung bis zum Betrachtungszeitraum vor, was je nach Sukzessionsstadium vom Gehölzanflug (aufkeimende Gehölze in der Etablierung) bis zu Vorwaldstadien reichen kann. Im Verlauf der Sukzession ist einerseits mit einem zunehmenden maximalen Wurzelhalsdurchmesser (WHD) sowie auch mit einer Zunahme des mittleren WHD auszugehen. Bereiche, auf denen sich Gehölze etablieren, sind in der Regel durch hohe Triebzahlen pro Quadratmeter und geringe WHD geprägt. Mit voranschreitender Größe und zunehmendem Standraumanspruch setzt eine Ausdünnung (self- thinning) ein, wodurch die Triebzahlen eine Reduktion erfahren. Der Bodenbedeckungsgrad durch die Baumkronen (in Prozent zur Bodenfläche angegeben) nimmt dabei gewöhnlich nicht ab, sondern wird durch eine geringere Triebzahl erreicht (Peringer, 2008). PerInger (2008) kann z.B. bei seinen Sukzessionsuntersuchungen von Schwarzerlen auf einem 210 Quadratmeter großen Sukzessionsstreifen, welcher repräsentativ den Übergang vom Offenland zum Vorwald darstellt, die Anzahl von 431 Erlen bestimmen. Davon sind $50 \%$ kleiner als $50 \mathrm{~cm}$ und die maximal aufgenommene Wuchshöhe beträgt $650 \mathrm{~cm}$. Durchmesser und Größe nahmen dabei in Richtung zum Vorwald im Durchschnitt kontinuierlich zu, während die Triebzahl pro Flächeneinheit abnimmt.

Der Sukzessionsprozess kann zusätzlich durch das Eindringen neuer Gehölzarten geprägt sein, wodurch bestehende Arten wieder verdrängt werden. Dieses kann die Artenzusammensetzung und zudem auch die Strukturen der Gehölzvegetation sehr vielfältig werden lassen. Die vorher dominierende Flora und Fauna des Offenlandes wird bei diesem Prozess in der Regel zunehmend zurück gedrängt (BöHMER \& BENDER, 2000). 


\subsubsection{Verbuschungen in Deutschland}

Auch in Deutschland hat der Anteil von Verbuschung betroffener Fläche nennenswerte Ausmaße angenommen. Man geht allein von ungefähr 15000 ha Magerrasen aus, die durch die Verbuschung unmittelbar bedroht sind (UNIVERSITÄT KASSEL, 2005). In den neuen Bundesländern erstrecken sich große Gebiete ehemaliger Truppenübungsplätze von mehreren zehntausend Hektar die durch eine mittlerweile fehlende Nutzung zunehmend der Gehölzsukzession unterliegen und ihren Offenflächencharakter verlieren (DEUTSCHER RAT FÜR LANDESPFLEGE, 1993; BRUNK ET AL., 2004).

\subsubsection{Biomasseaufkommen}

Durch den Gehölzaufwuchs entsteht im Rahmen der Verbuschungen durchaus auch ein beachtliches Biomassepotenzial. Mit zunehmender Altersstruktur und steigendem Verbuschungsgrad steigen die Gehölzmassen pro Flächeneinheit stark an, so dass bei der Entbuschung von Offenflächen beachtliche Mengen an Gehölzmaterial anfallen können. RAHMANN (2006) kann beispielsweise auf einer gleichmäßig verbuschten Magerrasenfläche mit einem Bodenbedeckungsgrad von $50 \%$ einen Gehölzaufwuchs von $5,2 t_{F M} /$ ha feststellen. Nach drei Jahren hat sich dieser auf 9,3t $\mathrm{t}_{\mathrm{FM}} /$ ha erhöht. Bei einer vorher vollständig entbuschten Teilfläche kann dazu nach drei Jahren ein Wiederbewuchs an Gehölzbiomasse von 9,9 $t_{\mathrm{FM}} /$ ha ermittelt werden. Durch den Frischmassebezug und fehlende Wassergehaltsangaben sind die Zahlen jedoch leider nur schwer vergleichbar.

In einem 94 ha großen FFH- Gebiet im Unteren Saaletal kann dazu im Rahmen einer Potentialstudie die Biomassevorräte, die bei der Entbuschung an Gehölzmaterial aufkommen würden, mit durchschnittlich 10,2 $\mathrm{t}_{\mathrm{TM}} /$ ha bestimmt werden. Die Bestandesstrukturen sind dabei sehr heterogen und es besteht eine erhebliche Variation der lokalen Biomasseaufkommen zwischen und innerhalb der einzelnen Flächenteile (MANN \& BÖHMER, 2008).

Genaue Abschätzungen zum Biomasseaufkommen und der Verbuschungsstruktur gestalten sich durch die sehr inhomogenen Bestandesdichten und Altersstrukturen auf den Flächen grundsätzlich als 
schwierig und sind nur sehr aufwendig zu ermitteln. Daten zu Verbuschungsstrukturen sind insgesamt nur wenige in der Literatur vorhanden, weil sich bisher noch keine wirtschaftliche Nutzbarkeit dieser Flächen abgezeichnet hatte. Zudem sind die bekannten aufgrund unterschiedlicher Bestimmungsmethoden nur schwer vergleichbar und spiegeln dabei oftmals nur ein unzureichendes Bild wider. Aktuell sind Forschungsprojekte initiiert worden, um geeignete Verfahren und Techniken zu entwickeln, um das Potential und die Art der natürlichen Biomasse genauer abschätzen zu können (z.B. „Energieholz und Biodiversität“ der NATURSTIFTUNG DAVID, WWW.NATURSTIFTUNG.DE ). Dieses trägt neben der Pflege auch dem gestiegenen Interesse an einer wirtschaftlichen Nutzung dieses Biomassepotentials Rechnung, da gestiegene Rohstoff- und Energiepreise zunehmend auch das Landschaftspflegeholz attraktiv werden lassen.

\subsubsection{Ziele und Restriktionen bei der Entbuschung von Offenflächenbiotopen}

Grundsätzlich wird als Ziel der Entbuschung von Offenflächenbiotopen eine Störung oder Beseitigung der Gehölzsukzession angestrebt, um Offenflächenbedingungen wiederherzustellen oder zu erhalten. Diese sind Voraussetzung für eine günstige Entwicklung von Flora und Fauna des Offenlandes bzw. zur Wiederherstellung eines vormals bestehenden Offenflächenbiotopzustandes. Die Wiederherstellung eines gehölzfreien Zustandes ermöglicht es dabei anschließend auch, dass angepasste Erhaltungsmaßnahmen, wie die Mahd oder eine extensive Beweidung, erst durchführbar werden. Die Entbuschung ist dabei Idealerweise so durchzuführen, dass die zu erhaltenen Flora und Fauna nicht geschädigt wird (DillenbuRger, 2005).

Eine Entbuschung beinhaltet neben dem reinen Schneiden und Abtrennen der Gehölze auch die Beseitigung des Schnittmaterials von der Fläche (Jedicke, 1996; Kollmann \& Staub, 1995; MünZel \& Schumacher, 1993; RAHMANN, 2000). Die Abfuhr des Materials sollte vor allem bei hohem Mengenaufkommen aus naturschutzfachlicher Sicht aus zweierlei Gründen 
erfolgen. Einerseits sollen Mulch- oder Reisigauflagen vermieden werden, da sie „erstickend“ auf die darunter befindliche schützenswerte Vegetation wirken. Der Lichteinfall wird behindert und es kann sich bei der Rotte des Materials ein Bodenparameter- und Feuchtigkeitsmilieu bilden, was für die Fläche untypisch wäre (z.B. pH- Wert, lokale Nährstoffakkumulation, längere Feuchtphasen). Andererseits wird durch die Abfuhr auch ein gewisser Aushagerungseffekt erzielt. Bisher ist keine Literatur bekannt, in der der Nährstoffentzug speziell durch Entbuschungsmaßnahmen untersucht wurde. Das Holz selbst ist insgesamt eher nährstoffarm, jedoch enthält die Rinde und das Reisig, welches bei dieser Art der "Vollbaum- Nutzung“ auch abgefahren wird, ein Vielfaches der im Holz gespeicherten Nährstoffmenge. Die höchsten Gehalte sind in den Blättern/ Nadeln gespeichert. WITTKOPF (2005) konnte für Nadel- und Laubbäume eine überschlägige relative Verteilung der Nährstoffe von 1 Teil im Derbholz (mit Rinde) zu 3 Teilen in Reisig/ Ästen $(<7 \mathrm{~cm})$ zu 9 Teilen im Laub/ Nadeln ermitteln. Der höhere Anteil in Reisig und Ästen zum Derbholz ist inm nach auf den höheren Rindenanteil zurückzuführen. Dieser Anteil könnte im Entbuschungsmaterial prinzipiell auch höher einzuschätzen sein, da bei Entbuschungen ein höherer Anteil an relativ dünnem Gehölzmaterial und Büsche mit hohem Reisiganteil zu erwarten sind.

Von technologischer Seite ist ein Abräumen der Fläche notwendig, um weitere Pflegemaßnahmen durch aufliegendes Reisig nicht zu behindern. Dabei ist das Interesse an dem Gehölzmaterial aus Entbuschungsmaßnahmen in den letzten Jahren durch die verstärkte Diskussion der Nutzung vorhandener Ressourcen stark gestiegen. Vor allem durch die Knappheit an Holzrohstoffen im Jahr 2007 und die damit stark gestiegenen Holzpreise ist das Biomassepotential von Landschaftspflegeholz weiter in das Blickfeld von Politik, Verwertern und Akteuren gerückt. Wurde noch vor wenigen Jahren oft von der Notwendigkeit zur kostenträchtigen „Entsorgung“ des Gehölzmaterials aus der Offenflächenpflege berichtet (RAHMANN, 2000), ist es nun eher möglich das Material einer sinnvollen Verwertung zuzuführen. Es wird mittlerweile auch berichtet, dass durch die Vermarktung Pflegekosten zu einem gewissen Teil kompensiert oder gar 
gedeckt werden können. Technisch und wirtschaftlich leichter zu bergendes Gehölzmaterial findet dabei natürlich bevorzugt Verwendung (NATURSTIFTUNG DAVID, 2010).

Die Wahl eines geeigneten Verfahrens zur Durchführung von Entbuschungsmaßnahmen auf Offenflächenstandorten stellt oftmals schon eine erste Hürde in der Praxis dar. Neben der Erfüllung naturschutzfachlicher Bedingungen zur Erreichung des Pflegeziels muss die Technik/ Technologie auch den vielfältigen Anforderungen des Standortes und der zu bearbeitenden Gehölze genügen. Hier sind allgemein z.B. die Befahrbarkeit der Fläche, zu bearbeitende Gehölzdurchmesser, besondere Anforderungen an den Schutz der Krautschicht sowie zusätzlich flächenspezifische Anforderungen zu nennen, welche in ihrer Kombination eine Vielzahl an Verfahrensmöglichkeiten ausschließen können. Nach Kromer \& Reloe (1991) spannen die Pflegeaufgabe (das Pflegeziel), der Standort und die Pflegefläche ein Wirkdreieck auf, in dessen Zentrum das zu wählende Verfahren steht .

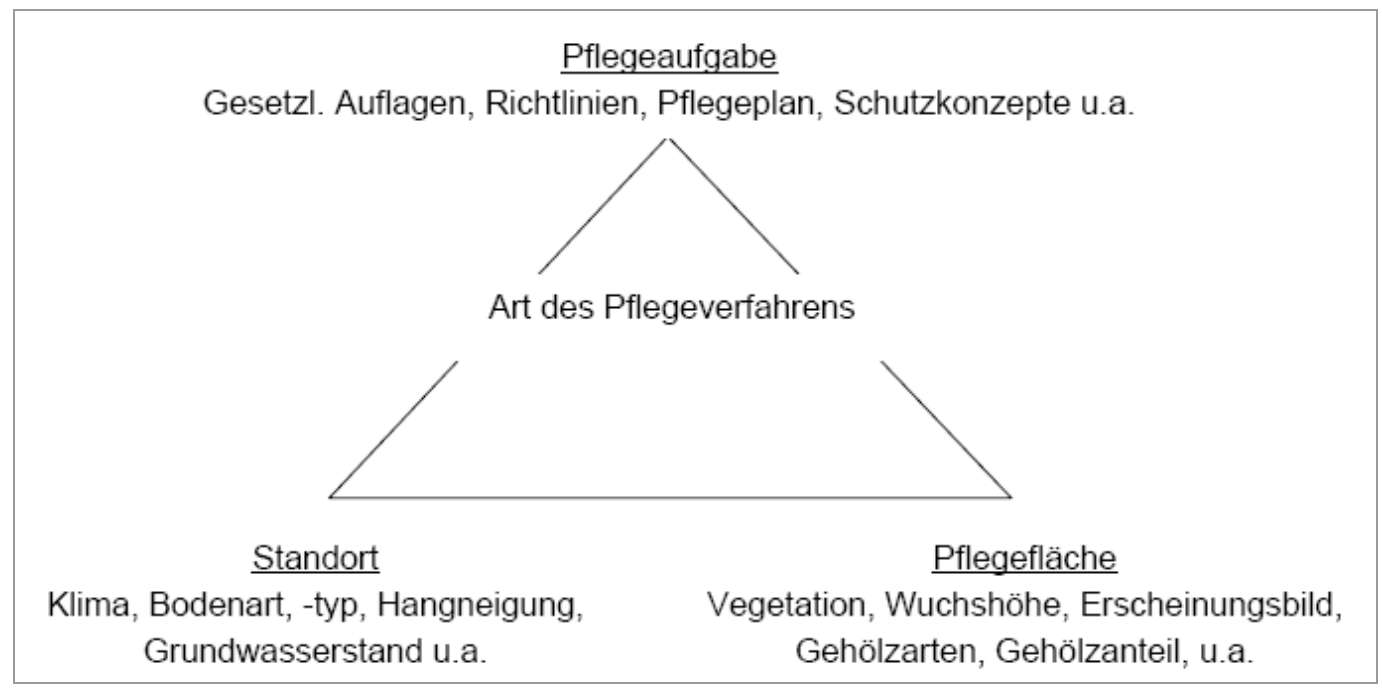

Abb. 2: Wirkdreieck der Verknüpfung von Pflegeaufgabe, Standort und Pflegefläche bei der Auswahl der Art des

Pflegeverfahrens (nach KROMER \& RELOE (1991), verändert)

Sind Verfahren bis zu diesem Punkt der Wahl geeignet, entscheiden letztlich auch die soziale und ergonomische Verträglichkeit, die Verfügbarkeit sowie die zu erwartenden Kosten über deren Vorzüglichkeit. Aufgrund dieser 
Komplexität ist es deshalb auch grundsätzlich nicht möglich, ein allgemein vorzügliches Entbuschungsverfahren zu benennen. Vielmehr muss für jeden Pflegeeinsatz abgewogen werden, welches Verfahren zum Einsatz kommen könnte, damit die angestrebten Pflegeziele unter Berücksichtigung technischer, naturschutzfachlicher, wirtschaftlicher sowie letztlich auch sozialer Gesichtspunkte weitestgehend erreicht werden können. Nicht selten müssen Kompromisse oder Verfahrenskombinationen eingegangen werden (JEDICKE, 1996).

\subsection{Pflegeverfahren und Techniken zur Entbuschung}

Um das Entwicklungsziel dieser Arbeit einordnen und bewerten zu können, werden im Folgenden die allgemein angewandten und in der Praxis etablierten Pflegeverfahren und Pflegetechniken sowie einige Spezialtechniken zur Entbuschung bzw. Offenhaltung dargestellt. Sie geben einen Überblick über den derzeitigen Stand der möglichen Aktivitäten des Naturschutzes und der Landschaftspflege zur Entbuschung von Offenlandbiotopen. Dabei sollen unter anderem auch die Einsatzgrenzen und die Schwächen der vorhandenen Landschaftspflegetechniken bzw. - verfahren bei der Entbuschung herausgestellt werden, um die Anforderungen des Arbeitsbereiches besser einschätzen zu können.

\subsubsection{Unmechanisierte Verfahren und Techniken}

Als „unmechanisiert" sollen an dieser Stelle entsprechende Verfahren und Techniken genannt werden, bei welchen die Mechanisierungen bei der Durchführung der Entbuschungsmaßnahme nicht im Vordergrund stehen. Die Verfahren selbst werden durch mechanische und technische Hilfsmittel unterstützt, sind jedoch nicht Kern der Maßnahme.

\subsubsection{Brennen}

Das eigentliche Ziel des kontrollierten Brennens ist ein „kaltes Mitwindfeuer", durch welches die oben aufliegende Spreu verbrannt wird, ohne die sich darunter befindliche Grasnarbe zu zerstören. Um die zu schützende Flora und Fauna weitestgehend $\mathrm{zu}$ schonen, wird das Brennen nach einer 
Kälteperiode vor der nächsten Vegetationsperiode eingesetzt. Diese so genannten „Winterfeuer" haben einen weit geringeren negativen Einfluss auf die sich in der Winterruhe befindlichen Tiere und Pflanzen als die "Sommerfeuer" während der Vegetationsphase. Die schonende Art des Brennens übt durch die geringe Hitzeentwicklung aber auch nur einen geringen Einfluss auf die unerwünschte Gehölzvegetation aus. Die entstehende Hitze kann zwar aufkeimende Gehölze weniger Zentimeter Wurzelhalsdurchmesser (WHD) schädigen, so dass sie verkümmern bzw. absterben, jedoch ist die Wirkung auf etablierte Bäume und Büsche nur gering. Das Verfahren ist somit im Bezug auf Gehölze eher geeignet einen Offenzustand zu erhalten als wiederherstellend bzw. entbuschend zu wirken (SCHREIBER ET AL., 2000).

In der Literatur sind je nach Standortbedingungen und Struktur der Brandflächen notwendige Aufwendungen zum Brennen von 42 bis $380 €$ / ha angegeben (PROChNow \& Schlauderer, 2002; MülleR \& Schaltegger, 2004). Eine direkte Leistung, die den Kosten gegenüber gestellt werden kann, entsteht nicht.

Das Abbrennen von Flächen ist nicht unumstritten. So ist in wissenschaftlichen Untersuchungen festgestellt worden, dass während der Maßnahme Nährstoffausträge in die Luft sowie auch verstärkt nach der Maßnahme Nährstoffausträge durch eine beschleunigte Mineralisation in den Unterboden entstehen können (FALK ET AL., 2004). Diese haben zwar einen gewünschten, aushagernden Effekt auf die Pflegefläche, sind jedoch bezüglich dem Schutz von Grundwasser und Luft bedenklich. (WANNER ET AL., 2004).

\subsubsection{Beweidung}

Zur Offenhaltung von Kulturlandschaften stellt die extensive Beweidung mit Haustierrassen ein Verfahren dar, welches sich am ehesten an die ursprüngliche Nutzung anlehnt.

Zur Entbuschungsleistung der Beweidung sind in der Literatur stark voneinander abweichende Resultate zu finden. Sie ergeben sich aus 
unterschiedlichen Standortfaktoren der vorliegenden Vegetation und der eingesetzten Nutztierarten. Allgemein wird Schafen und insbesondere Ziegen eine bessere Verbissleistung zugesprochen, weshalb sie zur Zurückdrängung von Gehölzen eher geeignet scheinen. Durch das „scharfe“ Verbeißen der Triebe und Blätter von Bäumen und Büschen sowie auch der Rinde werden diese nachhaltig geschädigt und können sogar absterben. Eine ausreichende Verbissleistung zur erfolgreichen Einschränkung des Gehölzaufwuchses ist jedoch nur durch eine entsprechend starke Beweidungsintensität zu erreichen, die nur bei hohen Besatzdichten und längeren Beweidungsintervallen gewährleistet werden kann. Eine Aushagerung der Flächen ist je nach Verfahrensanwendung, wie z.B. der Hütebeweidung, eingeschränkt möglich. In der Regel sind bei dem Verfahren der Beweidung Vor- bzw. Nacharbeiten notwendig, um einen ausreichenden Entbuschungseffekt erreichen zu können, was zu zusätzlichen Aufwendungen (Entbuschungsmaßnahmen) führt. Langfristig kann eine biotopverträgliche Beweidung nur eine Verbuschung unterdrückende bzw. Entbuschungsintervall verlängernde Maßnahme darstellen, da sie vornehmlich sich etablierende Gehölze weniger Zentimeter WHD sowie junge Triebe nachhaltig schädigen (BRUNK ET AL., 2004; RAHMANN, 2000; JEDICKE, 1996).

\subsubsection{Teilmechanisierte bis vollmechanisierte Verfahren und Techniken}

Die im Folgenden beschriebenen Techniken zur Entbuschung stellen den üblichen Technikeinsatz der Pflege dar. Neben der allgemeinen Eignung der Maschinen und Geräte ist ihre Vorzüglichkeit auch in der weiten Verbreitung in der Land- und Forstwirtschaft und der daraus resultierenden hohen Verfügbarkeit begründet.

\subsubsection{Manuelle/ Motormanuelle Entbuschung}

Die Entbuschung von Offenflächen mit handgeführten Geräten erfolgt vornehmlich auf nicht befahrbaren Flächen oder aus Gründen nicht vorhandener Alternativverfahren, da ihr Investitionsbedarf relativ gering ist. 
Sie ist jedoch auch verbunden mit einer geringen Flächenleistung sowie einer hohen körperlichen Belastung und Unfallgefahr. Für die Behandlung größerer Flächen ist dieses Verfahren nicht geeignet (DILLENBURGER, 2005).

Unterschieden werden kann zwischen den manuellen Geräten, wie z.B. Äxten, Heppen, Sägen und Astscheren sowie den motormanuellen, den Motorsägen und Motorfreischneidgeräten. Bei ersteren werden Büsche und Bäume mit der Hand bodennah abgeschlagen oder abgesägt, wobei sich der Einsatz durch die hohe körperliche Belastung auf geringere Gehölzstärken $(<7 \mathrm{~cm})$ beschränkt. Es wird dabei nicht das technische Umgangsvermögen bzw. eine Ausbildung wie bei den motormanuellen Geräten benötigt. Deren Einsatz sollte eher Fachkräften vorbehalten sein. Daneben zeichnet sich gerade bei mit der Flächenpflege beauftragten öffentlichen Einrichtungen (Naturschutzbehörden, Forstämtern) ein zunehmender Mangel an diesen ausgebildeten Arbeitskräften ab (LEVIN, 2004).

Mit Freischneidern können je nach Schneideinrichtung Gehölzstärken von bis zu $12 \mathrm{~cm}$ Durchmesser (Kreissägeblatt) gefällt werden. Beim Verwenden von Dickichtmessern am Freischneider können Stammdurchmesser bis $1,5 \mathrm{~cm}$, bei vorsichtiger und geschickter Handhabung auch bis $6 \mathrm{~cm}$ abgetrennt werden. Freischneider werden vornehmlich bei Gehölzanflug und dünnem Buschwerk eingesetzt, da sie bei hohen Stammzahlen pro Flächeneinheit und geringen Stammdurchmessern eine größere Flächenleistung ermöglichen.

Mit Motorsägen können auch Verbuschungen bzw. Bäume größeren Durchmessers abgetrennt werden. Hier ist im Prinzip kaum eine Durchmesserbegrenzung vorhanden. Aufgrund der gebückten Körperhaltung sollten sie jedoch auf größere Stammdurchmesser bzw. niedrigere Stammzahlen pro Flächeneinheit sowie auf das anschließende Zerkleinern der Gehölze beschränkt bleiben. In sensiblen sowie nicht oder schlecht befahrbaren Gebieten bietet sich der Einsatz der manuellen bzw. motormanuellen Entbuschung an, da auch bei schwierigen Standortbedingungen sehr selektiv und sehr schonend gearbeitet werden kann. Einen großen Aufwand stellt bei händischen Entbuschungsverfahren 
das Abräumen und Konzentrieren des Entbuschungsmaterials dar (JEDICKE, 1996).

Die Kosten der manuellen bzw. motormanuellen Entbuschung sind stark durch die Standortbedingungen sowie den Grad der Verbuschung bestimmt, so dass sich starke Schwankungen pro Flächeneinheit ergeben. Den Hauptanteil der Kosten nehmen dabei die Lohnkosten ein. Je nach Verbuschungsgrad und Schwierigkeit des Geländes sowie der Art der Materialräumung können Kosten von bis zu $10.000 € /$ ha entstehen. Kann das Entbuschungsmaterial verwertet werden, ist eine entsprechende Reduktion der Kosten möglich (PROCHNOW \& SCHLAUDERER, 2002).

\subsubsection{Mulchen}

\section{Landwirtschaftliche Mulchgeräte}

Mit landwirtschaftlichen Mulchgeräten an Traktoren können befahrbare Offenstandorte wirkungsvoll und vollmechanisiert gepflegt werden. Prinzipiell sind die Geräte nur zur Zerkleinerung von Kraut- und Grasvegetation konzipiert, die nach dem Zerkleinerungsprozess auf der Fläche breit verstreut wird, wo sie verrottet (JEDICKE, 1996).

Mit Mulchgeräten lassen sich je nach eingesetztem Mulchwerkzeug auch Gehölzaufwüchse bis zu einem Wurzelhalsdurchmesser von $3 \mathrm{~cm}$ Abschlagen und Zerkleinern, wodurch vor allem Initialstadien der Verbuschung effektiv zurückgedrängt werden können. Da das Mulchmaterial nicht kostspielig entsorgt werden muss, wie es z.B. bei der Mahd aufgrund einer fehlenden Verwertbarkeit nicht unüblich ist, wird das Verfahren häufig auch als alleinige Pflegemaßnahme angewandt. Eine Aushagerung der Flächen ist durch den Verbleib des Materials auf der Fläche dann jedoch nicht möglich, was entsprechend der Pflegeziele naturschutzfachlich abgewogen werden muss (SCHREIBER ET AL., 2000). Durch einen Saugeffekt der Werkzeuge und eine ungünstige Maschineneinstellung zum Boden können schädigende Wirkungen auf die Flora und vor allem die Fauna der Krautschicht entstehen (LÖBBERT, 2001). 
Ein Grund für den verstärkten Einsatz des Mulchverfahrens ist die weite Verbreitung derartiger Maschinen in der Landwirtschaft. So sind es oftmals Landwirte, die die Mulcharbeiten ausführen und damit die Auslastung der vorhandenen Geräte erhöhen.

\section{Forstwirtschaftliche Mulchgeräte}

Einen weiteren Typ von Mulchgerät stellen die schweren Forstmulchgeräte dar, die auch Gehölze mit Wurzelhalsdurchmessern über $20 \mathrm{~cm}$ abschlagen und zerkleinern können. Sie arbeiten nach dem gleichen Prinzip wie die landwirtschaftlichen Mulchgeräte, wobei ihr Aufbau jedoch weitaus massiver und robuster ist.

Die Arbeitsweise beim Forstmulchereinsatz besteht in der Regel aus zwei Schritten- einem Fäll- und einem Zerkleinerungsvorgang. Vor allem in diesem zweiten Arbeitsschritt arbeiten die Forstmulchgeräte sehr nah an oder gar im Oberboden, wodurch das zerkleinerte Holzmaterial auch in den Boden eingearbeitet und mit ihm vermischt werden kann (BLOCK, 2004).

In sensiblen Gebieten führen die auftretenden Beschädigungen zu nicht tolerierbaren Eingriffen in die Krautschicht und der dort befindlichen Flora Fauna. Bei stark degenerierter Krautschicht kann er jedoch ein praktikables Mittel sein, um Ruderalstandorte zu erstellen (DILLENBURGER, 2005).

Eine Möglichkeit zur Materialaufnahme und somit zur Aushagerung der Standorte bzw. späteren Nutzung des Materials besteht bei konventionellen Forstmulchgeräten aufgrund des Arbeitsprinzips ebenso wenig wie bei den landwirtschaftlichen Ausführungen.

\subsubsection{Fällgreifer}

Durch die zunehmende Verwertung des Gehölzaufwuchses in den letzten Jahren haben sich Fällgreifertechniken etablieren können. An Auslegerkränen von Baggern, Forsterntemaschinen, Schleppern oder Rückewagen können Fällgreifer montiert werden, mit denen Bäume und auch Büsche gefasst und mit scheren- bzw. klingenartigen Werkzeugen abgetrennt werden können. Je nach System können Stammdurchmesser von bis zu $30 \mathrm{~cm}$ bearbeitet werden. Dabei haben sie keine entastende Funktion. 
Innerhalb der Kranauslage ist es möglich auch nicht befahrbare Flächen zu erreichen und dort Gehölze zu entfernen. Die abgetrennten Gehölze können anschließend auf Haufen abgelegt werden, von wo sie anschließend geräumt werden können (STÜBIG, 2000; AfFENZELLER \& STAMPFER, 2007).

Aufgrund des Arbeitsprinzips „Fassen- Fällen- Ablegen“ ist die Verwendung von Fällgreifersystemen grundsätzlich erst ab einem gewissen Stammdurchmesser sinnvoll, da jeder Stamm bzw. Wurzelstock einzeln bearbeitet werden muss. Die Verwendung von Fällgreifern bei hoher Triebzahl pro Quadratmeter und überwiegend dünnen Trieben kann somit nicht wirtschaftlich realisiert werden, da die Flächenleistung bezüglich beider Faktoren überproportional abnimmt (Stück-Masse-Gesetz) und die spezifischen Verfahrenskosten stark ansteigen. Aussagen von Praktikern zur Folge sollte der überwiegende Teil der zu kappenden Gehölze einen mittleren Wurzelhalsdurchmesser von über $10 \mathrm{~cm}$ besitzen (HEIKE, 2008). Technisch sind grundsätzlich auch Durchmesser bis auf wenige Zentimeter bearbeitbar, deren Anzahl sollte aber weitmöglich gering sein. So genannte Fäller-Bündler Systeme sind speziell für die Beerntung von dünneren Stammdurchmessern konzipiert worden. Sie sind eine Sonderbauform der Fällgreifer mit höhere Arbeitsleistung, die bis zu sechs oder mehr Triebe sammeln können, bevor diese abgelegt werden müssen. Arbeitszeitstudien und Erfahrungen aus dem Forstbereich geben hierzu einen Durchmesser im Bereich von 5- $10 \mathrm{~cm}$ an, in denen der Einsatz bei entsprechend höheren Triebzahlen pro Quadratmeter arbeitswirtschaftlich noch zu rechtfertigen sein soll. Grundsätzlich lassen sich Fällgreifer durch diese Voraussetzungen nur auf Flächen sinnvoll einsetzen, deren Gehölzsukzession schon stark vorangeschritten ist und ein entsprechend hoher Anteil an größeren Stammdurchmessern vorliegt (Lit.: GRIMM, 1980; KüPPERS, 1997; STÜBIG, 2000; GABriel, 2003; AfFenzeller \& Stampfer, 2007; Cremer ET AL., 2007)

\subsubsection{Spezialtechniken}

Neben den oben beschriebenen, allgemein verbreiteten Entbuschungstechniken kommen auch speziellere bzw. unkonventionellere zum Einsatz. Sie wurden meist für gesonderte Einsatzzwecke der 
Landschaftspflege entwickelt und konnten sich bisher nicht verbreitet durchsetzen. Oft existieren nur wenige Exemplare. Sie sind deshalb nicht flächendeckend verfügbar und könnten höchstens überregional zur Verfügung stehen.

\subsubsection{Mäh- und Laderaupe}

Auf Basis von den von Skipisten bekannten Raupenfahrzeugen („PistenBullys“) wurden verschiedenartige Aufbauten zur Landschaftspflege entwickelt (z.B. Firma mera-Rabeler). Die Raupenfahrzeuge erlauben trotz hoher Beladungsgewichte durch ihre großzügige Kettenauflagefläche geringe spezifische Bodendrücke ab $0,036 \mathrm{~kg} / \mathrm{cm}^{2}$ bis $0,170 \mathrm{~kg} / \mathrm{cm}^{2}$ und werden mit einer Antriebsleistung von bis zu 346 kW ausgestattet. Die zusätzlich hohe Steigleistungs- und Schräglagentauglichkeit ermöglicht ihnen in schwierigem und vor allem auch nassem Gelände vielerlei Vorzüge bei Landschaftspflegearbeiten hinsichtlich Bodenschonung und Leistungsfähigkeit. Aufgrund des verhältnismäßig großen Bauraums hinter der Kabine können Aufbauten zur Materialbergung angebracht werden.

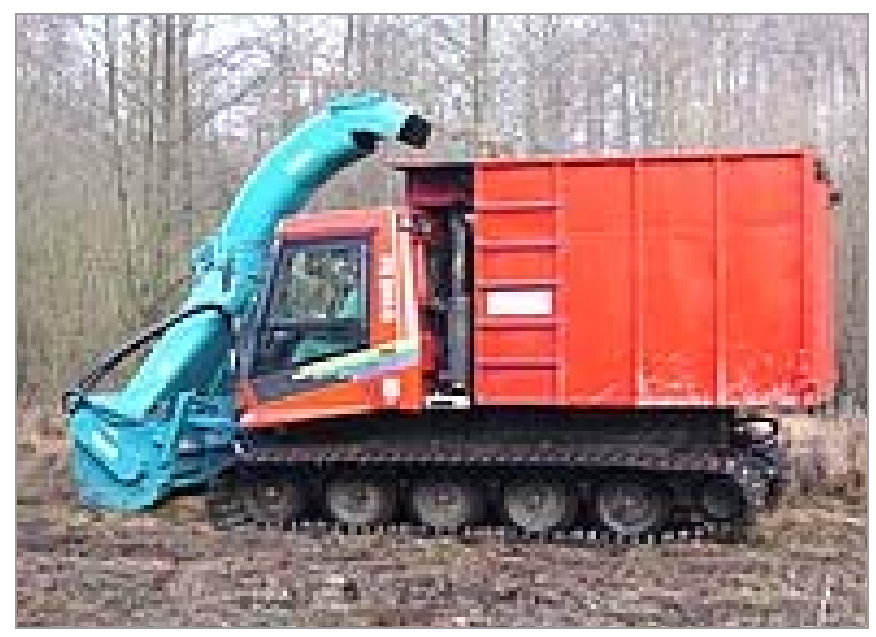

Abb. 3: Mäh- und Laderaupe des Herstellers „mera Rabeler“; Im Besitz des Landschaftspflegeunternehmens Meyer-Luhdorf, Winsen/ Luhe (MEYER-LUDORF, 2009)

Die in Abb. 3 dargestellte Gerätekombination ist z.B. zur Entbuschung von Offenflächen entwickelt worden. Sie besteht aus einem im Frontanbau an ein Raupenfahrzeug aufgehängtem Wurfschlegelmäher mit Auswurfkrümmer und einem hinter der Kabine aufgesattelten Kippcontainer. Die Maschine 
arbeitet auf einer Breite von $2 \mathrm{~m}$ und mit dem Schlegelgerät können Bäume und Büsche bis zu einer Stärke von $8 \mathrm{~cm}$ Durchmesser in einem Arbeitsgang entfernt und das Material gleichzeitig von der Fläche geräumt werden. Das durch die Schlegel abgetrennte und grob zerkleinerte Material wird dabei über die Kabine hinweg in den Container befördert und kann an einem Sammelplatz entladen werden. Durch die gleichzeitige Aufnahme von nassem Gras- und Krautmaterial sowie der unspezifischen Zerkleinerung ist eine spätere thermische Verwertung des Häckselgutes nur schwer zu realisieren. Die große und hinsichtlich der Bodenschonung vorteilhafte Auflagefläche der Ketten birgt für den Praxiseinsatz ein zusätzliches Problem. Der Transport der Maschine zum Einsatzort kann nur sehr bedingt per Achse erfolgen und erfordert aufgrund der Breite der Maschinen von über 2,55 m grundsätzlich den Einsatz von speziellen Transportfahrzeugen mit den entsprechenden Genehmigungen (RABELER, 2008).

\subsubsection{2. „Rotorsägen“}

Rotorsägen bestehen aus einem waagerecht angeordneten Sägeblatt an Auslegern von Baggern oder Kränen sowie auch an der Drei- Punkt- Aufhängung von Traktoren, mit dem die Gehölze bodennah abgetrennt werden. Je nach Ausführung können Gehölze mit einem Stammdurchmesser von bis zu $20 \mathrm{~cm}$ gefällt werden. Zur Beseitigung müssen die Gehölze in einem weiteren Arbeitsschritt von der Fläche geräumt werden, da sie in dem Vorgang ausschließlich gefällt werden und danach in der Regel unkontrolliert zu Boden fallen. Durch die begrenzten Arbeitsbreiten der Sägeblätter von maximal $1,5 \mathrm{~m}$ sind zur großflächigen Entbuschung enorme Aufwendungen zum Schwenken der Ausleger erforderlich, weshalb dieses Verfahren auch aufgrund der geringen Flächenleistungen nur bedingt geeignet ist (GRIMM, 1980).

Eine nennenswerte Variante der Rotorsägen stellt eine massive Ausführung dar, deren $4 \mathrm{~cm}$ starke und $1 \mathrm{~m}$ Durchmesser messende Rotorscheibe mit Hartmetallmeißeln bestückt ist. Die Rotorsäge wird an einen Kompaktlader oder in die Drei- Punkt- Hydraulik eines Traktors eingehängt und schlägt die Gehölze zerfasernd und bodennah ab. Neben der Robustheit der Technik 
kann ein derartiger Trennvorgang bei der Entbuschung vorteilhaft sein, da der zerfasernde Schnitt die Wurzelstöcke der Gehölze stärker schädigen und die Wiederaustriebskraft herabsetzen kann (LEINONEN, 2007).

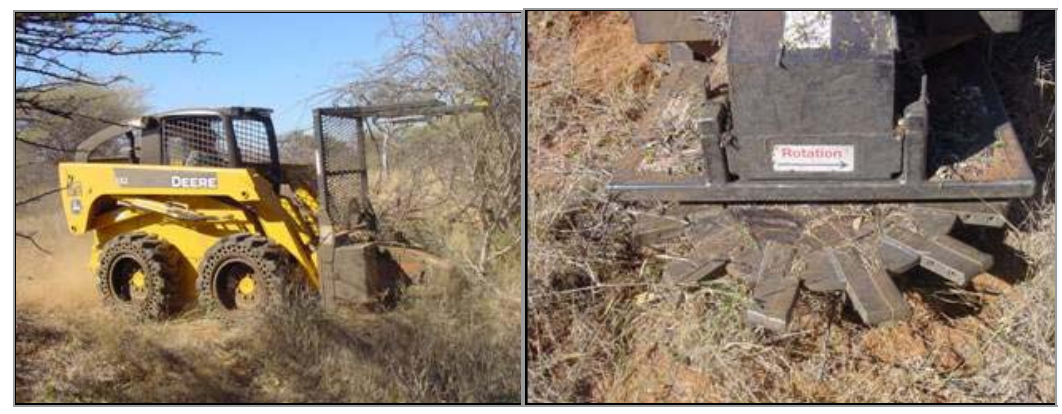

Abb. 4: Kompaktlader beim Fällen von Büschen in Namibia (links) mit robust ausgeführter Rotorsäge (rechts) (LEINONEN, 2007)

Ein offensichtlicher Nachteil bei einer derartigen Ausführung ist, dass die Wurzelstöcke einzeln angefahren werden müssen. Durch die vielen Drehund Fahrvorgänge wären vor allem in sensiblen Gebieten übermäßige Schädigungen des Oberbodens zu befürchten.

Bei Untersuchungen im südafrikanischen Buschland werden Fälleistungen von bis $2,5 \mathrm{ha} / \mathrm{h}$ angegeben (LEINONEN, 2007). Leider fehlen detailliertere Angaben zur Pflanzenanzahl sowie zur Alters- und Gehölzstruktur beim Einsatz dieses Gerätes, so dass die Leistungsangabe nur bedingt zu beurteilen ist.

\subsubsection{Forstmulcher mit Überladefunktion}

Eine neue Technik zur Entbuschung stellt der Forstmulcher mit Überladefunktion dar. $\mathrm{Er}$ ist derzeitig nur als Prototyp bzw. Vorserienmaschine erhältlich, so dass bisher keine belastbaren Erfahrungen bzw. wissenschaftlichen Untersuchungen hinsichtlich der Einsatzfähigkeit solch eines Systems in der Landschaftspflege vorliegen. Das Arbeitsprinzip gleicht den konventionellen Forstmulchern (siehe Kap. 3.2.2.2), jedoch sind diese mit einer Überladefunktion ausgestattet, mit der das geschredderte Material verladen werden kann. Der Vorteil dieser Technik ist sicherlich in seiner Robustheit und in seinem breiten Einsatzspektrum hinsichtlich der Gehölzstrukturen zu finden. Der Einsatz birgt jedoch auch die Nachteile des 
Mulchersystems bezüglich des Krautschichteingriffs und der unspezifischen Zerkleinerung. Beim Mulcher wird das Material nur grob zerschlagen bzw. zerschreddert und die Aufnahme von Boden- und Gras- bzw. Krautmaterial kann nur bedingt verhindert werden. Das resultierende Material des Überlademulchereinsatzes wird nur einer minderwertigen Verwendung zugeführt werden können, es sei denn, dass es durch Siebung etc. aufgewertet wird. Grundsätzlich ist eine Materialabfuhr jedoch möglich. Das Maschinengewicht Z.B. des AHWI- Überlademulchers ist mit $5340 \mathrm{~kg}$ als hoch einzustufen, vor allem da noch das Gewicht eines entsprechendes Antriebsfahrzeuges mit einer empfohlenen Leistung von 175 bis $295 \mathrm{~kW}$ hinzukommt (SCHMIDT, 2008; AHWI MASCHINENBAU GMBH, 2008).

\subsubsection{Räumung und Bergung des Entbuschungsmaterials}

Gehölzmaterial, welches nicht gleichzeitig geborgen oder derart aufgearbeitet wird, dass es bzw. dessen Residualmasse auf der Fläche verbleibt, wie es z.B. beim Brennen oder beim Mulchen der Fall ist, muss von der Fläche beseitigt werden. Dieses erfolgt in weiteren vom Fällvorgang getrennten Arbeitsschritten. Man spricht dann aufgrund der Entkoppelung vom Fällvorgang von mehrphasigen Arbeitsverfahren.

\section{Räumung}

Die Räumung der Gehölze dient allein dem Zweck die Krautschicht der entbuschten Fläche freizulegen und weiter folgende Pflegemaßnahmen nicht zu behindern oder zu erschweren. Eine Verwertung ist dann in der Regel aufgrund der Materialbeschaffenheit, der fehlenden Bergungsmöglichkeit, eines fehlenden Verwertungsweges oder aus ökonomischen Gründen nicht möglich. Gängige Praxis ist es, das Material zu Haufen aufzuschichten, wo es je nach Gehölzaufkommen sowie auch nach naturschutzfachlicher Abwägung entweder als Rottehaufen auf der Fläche verbleibt oder dort verbrannt wird (JEDICKE, 1996). Das Konzentrieren des Materials erfolgt entsprechend der Einsatzmöglichkeiten (wie Befahrbarkeit, Technikverfügbarkeit) per Hand oder mit technischen Mitteln (z.B. Traktor mit Frontlader- oder Drei- Punkt- Reisiggabel, Forstladewagen mit Kran). 
Da aus der ausschließlichen Räumung kein verwertbares Produkt entsteht, können die Kosten nicht durch einen entsprechenden Gegenwert der Maßnahme aufgefangen oder minimiert werden (JEDICKE, 1996).

\section{Bergung und Verwertung}

Bei der Bergung des Materials werden die Gehölze aufgearbeitet oder natürlich belassen von der Fläche abgefahren und einer Verwertung bzw. Weiterverarbeitung zugeführt. Die Aufarbeitung erfolgt aufgrund der im Vergleich zu Waldholz schlechteren Materialbeschaffenheit üblicherweise zu Holzhackschnitzel- oder Schreddermaterial, welche z.B. zu Heizzwecken oder zur Kompostierung genutzt werden können. Untersuchungen zur Materialqualität ergaben je nach Ausgangsmaterial durchaus auch eine Vergleichbarkeit mit Waldrestholz (WIEGMANN, 2007; CREMER ET AL., 2007; DEUTSCHER VERBAND FÜR LANDSCHAFTSPFLEGE E.V., 2008).

Die Bergung beginnt analog der Räumung entweder mit der Abfuhr des Materials zu einem zentraleren Verarbeitungsplatz oder mit der direkten Verarbeitung des Gehölzmaterials auf der Fläche. Auf der Fläche werden meist kleine Häckselgeräte mit Eigen- bzw. Traktorantrieb eingesetzt. Sie haben relativ geringe Produktionsleistungen. Die Beschickung erfolgt meist per Hand oder auch mit Forstkränen. Der Abtransport der produzierten Hackschnitzel erfolgt zumeist mit kleineren Transportfahrzeugen, vor allem, wenn die Befahrbarkeit der Flächen aus Bodenschutzgründen und die Überladehöhe der eingesetzten Häcksler begrenzt sind (JEDICKE, 1996).

In selteneren Fällen werden Großmaschinen wie spezielle Selbstfahrer mit einem Häcksleraufbau mit Zwischenbunker aus dem Forstbereich zum direkten Häckseln auf den Flächen eingesetzt. Diese sind zwar an schwierige Geländeverhältnisse und schonende Arbeitsweise angepasst, jedoch im Betrieb aufgrund einer hohen Leistungsfähigkeit recht kostenintensiv. Ihr Einsatz lässt sich erst bei entsprechend großer Gesamtmenge und entsprechend konzentriertem Gehölzmaterial arbeitswirtschaftlich sinnvoll durchführen. Arbeitszeitstudien aus dem Forstbereich gehen je nach Logistik von einem Brusthöhendurchmesser (BHD) von ca. 10-15 cm aus, welcher im Durchschnitt auf der Fläche vorliegen sollte, damit eine Kostendeckung 
des Verfahrens erreicht werden kann. Wird das Gehölzmaterial unbearbeitet von der Fläche zu einem geeigneten Verarbeitungsplatz transportiert, ist es möglich auch entsprechend größere Häckselaggregate einzusetzen, die das dort konzentrierte Gehölzmaterial stationär zerkleinern (FELLER ET AL., 1998; CREMER, 2008; WitTKOPF ET AL., 2003).

Eine Zwischenlagerung des Gehölzmaterials abseits der Fläche ermöglicht es, dass das in der winterlichen Pflegeperiode geschlagene Material über eine gewisse Zeit vorgetrocknet werden kann, bevor es zerkleinert wird. Die so verminderten Feuchtgehalte führen zu einem gesteigerten Heizwert und einer verbesserten Lagerstabilität, was derart produzierte Landschaftspflegehackschnitzel aufwerten kann (BAUER, 2005). Sind geeignete Plätze zur Zwischenlagerung nicht vorhanden, kann das Gehölzmaterial auch unbearbeitet zum Ort der Verwertung transportiert und dort verarbeitet werden. Das größere Transportvolumen von unzerkleinertem Buschmaterial sowie der größere Umschlagaufwand sind jedoch sehr kostenintensiv, weshalb die Zerkleinerung und Aufarbeitung vor dem Transport grundsätzlich vorgezogen wird (JEDICKE, 1996). 


\subsection{Technikprinzipien der Holzzerkleinerung}

Zur Zerkleinerung von Holz werden schneidende und reißende Verfahren eingesetzt. Die reißenden, sogenannte Schredderverfahren, sollen hier nicht betrachtet werden, da ihr spezifischer Leistungsbedarf um das 5- 10- fache höher ist als der der schneidenden Verfahren (FIRUS \& BELTER, 1998) und sie durch ihren massiven Aufbau für den mobilen Einsatz eher ungeeignet sind.

Bei den schneidenden Häckselverfahren haben sich drei Maschinenprinzipien etabliert: der Trommelhacker, der Scheibenradhacker und der Schneckenhacker. Ihr Arbeitsprinzip ist in Abb. 5 schematisch abgebildet.

\begin{tabular}{|l|l|l|}
\hline Hockerbouart & Messerzohl & Einzugort \\
\hline
\end{tabular}

Abb. 5: Gängige Technikprinzipien zur Zerkleinerung von Holz (BRUSCHE, 1983, verändert)

Die Zerkleinerung erfolgt bei allen durch rotierende Messer, welche um eine gelagerte Achse eine Kreisbahn vollziehen, in die das zu zerkleinernde Holz idealerweise annähernd quer zur Faserrichtung eingebracht wird. Beim Auftreffen der Messer auf das Holz lassen die Messer durch die Schnittbewegung eine definierte Schnittfläche durch den Holzquerschnitt entstehen. Dabei bewirken Schubkräfte ein Aufspleißen in Faserrichtung, so das einzelne Späne zur Faserrichtung abgelöst werden (FIRUS \& BELTER, 1995A; MAIER, 1983). 


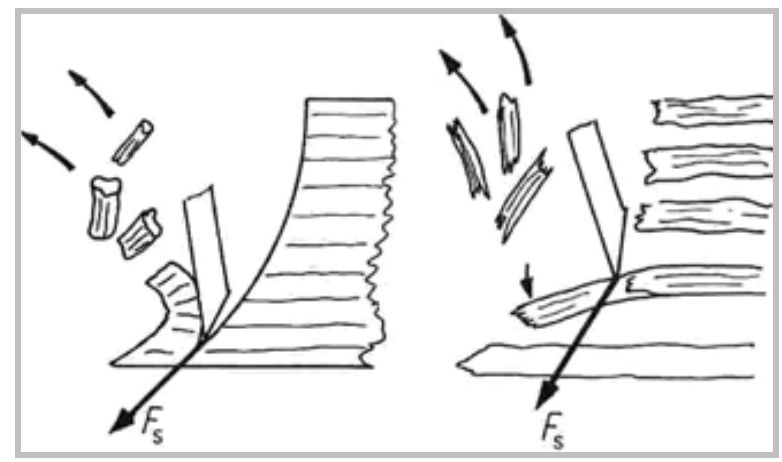

Abb. 6: Entstehung von Hackschnitzeln im Trommelhacker (MAIER, 1983)

Trommelhacker arbeiten dabei mit tangential an einem Rotor umlaufenden Messern. Dabei wird das Hackgut im rechten Winkel zur Trommelachse zugeführt. Trommelhacker lassen im Verhältnis zur Einzugsgroße eine relativ kleine Hacktrommel zu und ihr Aufbau ist kompakt. Die Hackschnitzelgröße wird bei innen über die Messerzahl am Umfang, der Rotordrehzahl sowie der Zuführgeschwindigkeit gesteuert. Dabei ist zur Herstellung gleichmäßiger Hackschnitzellängen eine kontinuierlich gleiche Zuführgeschwindigkeit des zu zerkleinernden Materials zu garantieren. Vor allem bei der Zerkleinerung von Reisig sollten sie mit einer gesteuerten Zuführung versehen sein, da ein idealer Einzug bzw. eine ideale Zerkleinerung nicht sicher gestellt werden kann (DENKINGER, 2004).

Bei Scheibenradhacker sind die Zerkleinerungsmesser auf einer zentrisch rotierenden Scheibe angeordnet, auf der sie mehr oder weniger radial zur Achse ausgerichtet sind. Das Material zur Zerkleinerung kann im rechten Winkel zur Scheibenachse oder auch abweichend dazu zugeführt werden, wodurch ein Selbsteinzugseffekt ausgelöst werden kann. Das Material wird im Zuführprozess gegen die Hackscheibe gepresst und durch die aus der Scheibe stehende Weite der Messer, lässt sich die Hackschnitzellänge einstellen. Dabei muss jedoch auch die entsprechende Zuführgeschwindigkeit garantiert werden. Da zum Zentrum der Scheibe die Messergeschwindigkeit abnimmt, kann nur auf dem äußeren Drittel der Scheibe zerkleinert werden. Aus diesem Grund sind Scheibenradhacker baulich verhältnismäßig groß und müssen aus Stabilitätsgründen bezüglich der Hackscheibe verhältnismäßig schwer ausgeführt werden. Um vor allem 
bei Reisigmaterial eine definierte Zerkleinerung zu erreichen, sollten nah zum Messer Zuführaggregate installiert sein. Scheibenradhacker neigen bei Reisig zu einem unkontrollierten Einziehen des Materials bei dem vor allem dünne Äste nur geknickt und nicht geschnitten werden (HAKKILA, 1989; BRUSCHE, 1983; JONES, 1997).

Der Schneckenhäcksler oder auch Schneckenhacker zerkleinert das Holz mit einer konischen Häckselschnecke, welche mit einer geschärften Schneide bestückt ist. Diese verläuft gewindeartig um den Rotorkörper. Die Zuführung erfolgt bei Standardschneckenhackern axial zur Rotorachse (Abb. 7).

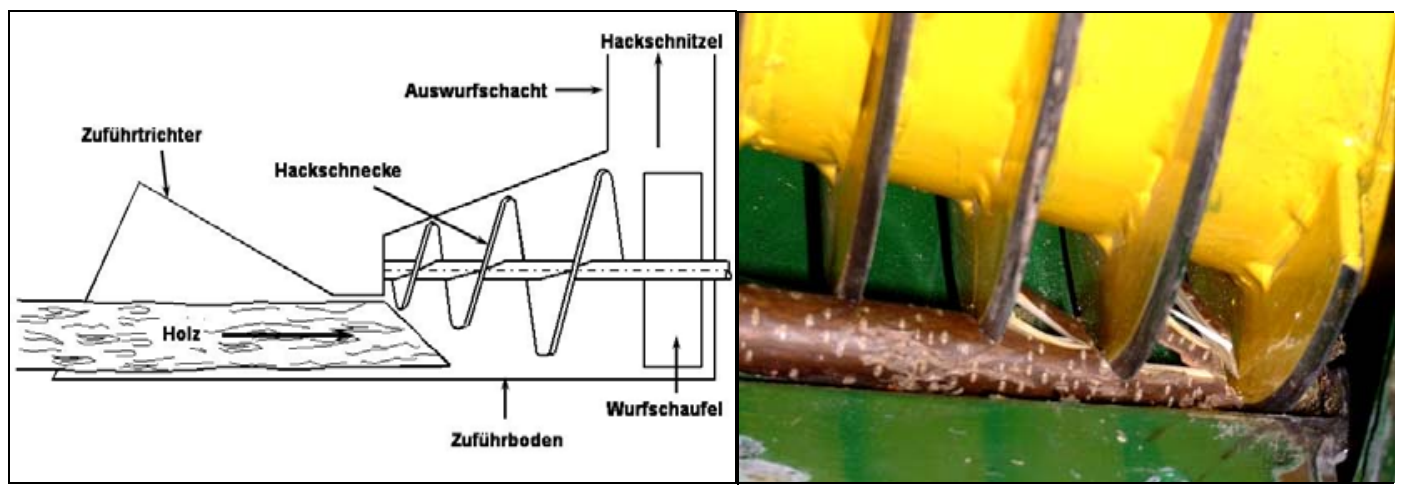

Abb. 7: Einzugsprinzip des Schneckenhackers, links: schematisch nach BRUSCHE (1983); rechts: Spanbildung bei der Zerkleinerung

Beim Auftreffen des Holzes auf der Schneide schneidet diese durch den größer werdenden Konus in das Holz ein. Durch die Schraubwirkung wird das Holz fortwährend weiter eingezogen und durch den großer werden Konuskörper dringt die Schneide tiefer in das Holz ein. Dabei schert sie Hackstücke zwischen den Schneiden aus dem Holz heraus. Beim Eindringen in das Holz wirken radiale Kräfte der Häckselschnecke auf den Zuführboden und tangentiale Kräfte durch die Drehbewegung der Schnecke. Um ein Mitdrehen des Holzes zu verhindern ist bei einem Schneckenhacker ein Widerlager in der Zuführöffnung angebracht. Dieses bildet in der Zuführöffnung den sogenannten „Häckselspalt". Schneckenhacker sind aufgrund ihres Zerkleinerungsprinzips selbsteinziehend und benötigen in der Regel keine Zuführaggragate für den Zerkleinerungsprozess. 
Zur Beschreibung einzelner Komponenten und Geometrien am Häckselrotor lassen sich Begrifflichkeiten aus der Schraubentheorie heranziehen (Abb. 8; Abb. 9).

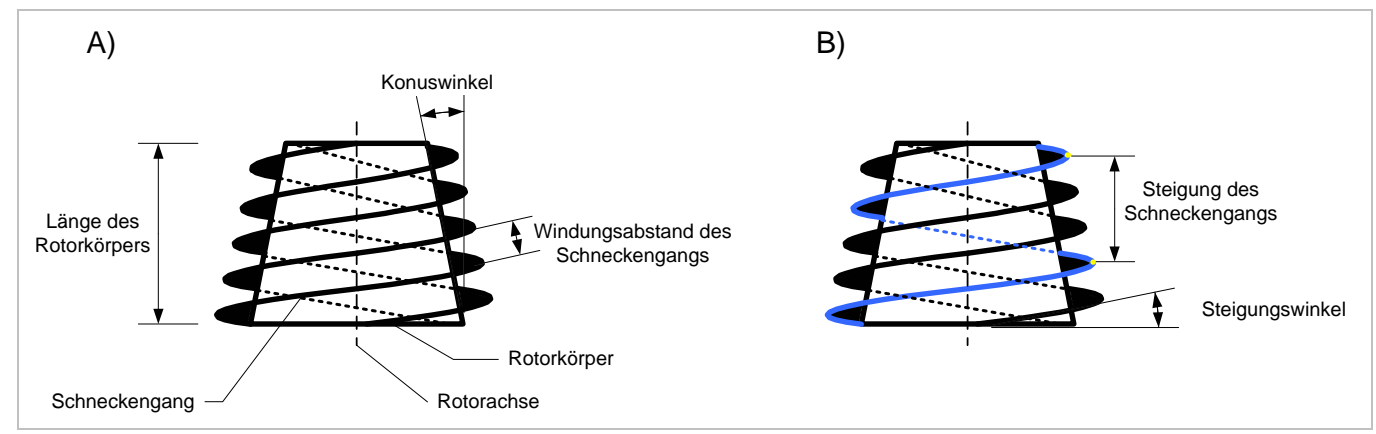

Abb. 8: Bezeichnung von Komponenten und Geometrien am Schneckenhacker; B) dreigängiger Rotor (Quelle: Wegener, J.-K., Göttingen)

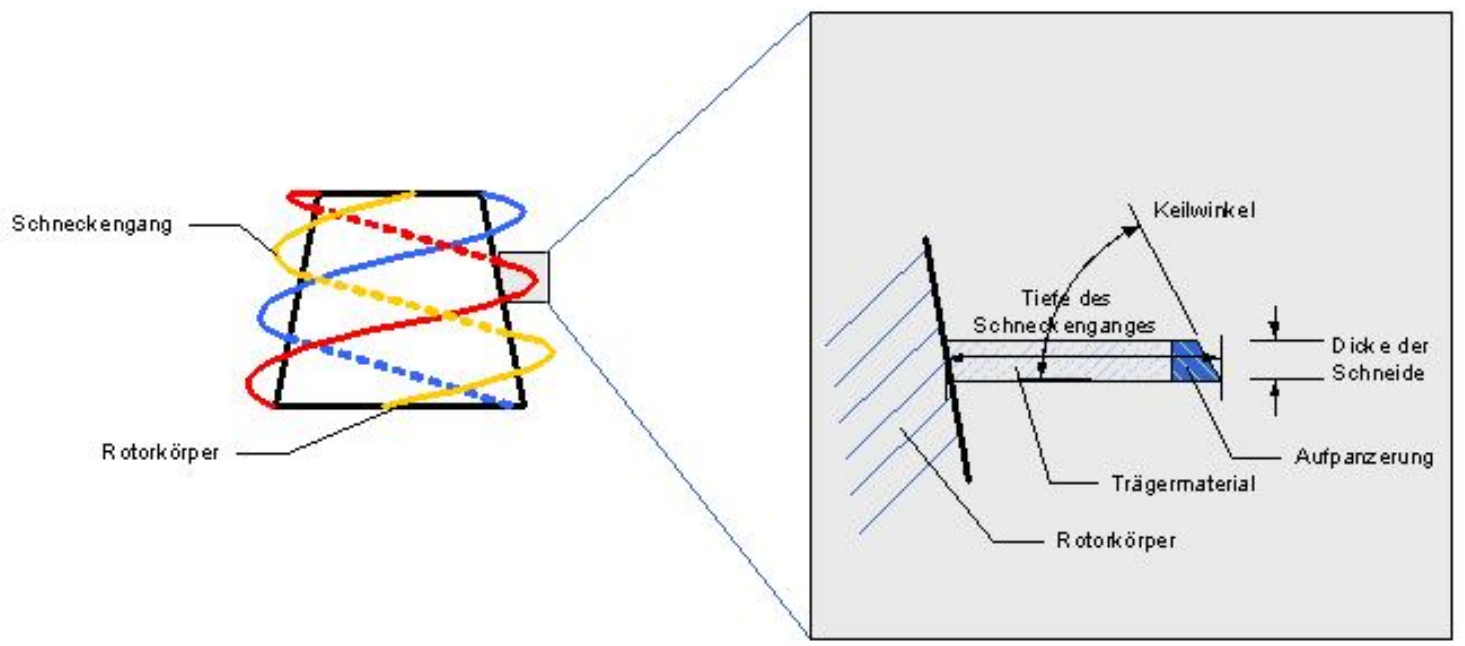

Abb. 9: Bezeichnungen von Komponenten und Geometrien am dreigängigen (rot, blau, gelb) Schneckenhacker und der Schneckenschneide (Quelle: Wegener, J.- K., Göttingen)

Die Steigung der Windung(en) gibt bei entsprechend der Drehzahl des Rotors die Einzugsgeschwindigkeit und der Windungsabstand die Hackstückgröße vor. Die theoretische Einzugsgeschwindigkeit folgt dabei der allgemeinen Formel aus der Schraubentheorie: 


$$
v_{E t}=n * P_{h}
$$

Gleichung 1: Berechnung der theoretischen Einzugsgeschwindigkeit $\left(v_{\mathrm{Et}}\right.$ in $\left.\mathrm{cm} / \mathrm{s}\right)$ von Schneckenhackern mit: $n=$ Drehzahl $(U / \mathrm{s}) ; P_{h}=$ Steigung $(\mathrm{cm} / U)$

Wie BRUSCHE (1983) und NURMI (1986) in ihren Arbeiten schon feststellen, ist die Einzugsgeschwindigkeit sowie auch die Hackstückgröße um einen gewissen Faktor geringer. Dieser kann jedoch von ihnen nicht quantifiziert werden, sondern nur in Versuchen zu bestimmten Häckselschnecken ermittelt werden. Mit Schneckenhackern lassen sich Hackschnitzel bis zu 150 mm Länge herstellen.

\subsection{Qualitätsparameter von Holzhackschnitzeln}

Holzhackschnitzel stellen eine Aufarbeitungsform von Holzrohstoffen dar. Ziel ist es, aus inhomogenen Ausgangsstoffen ein relativ homogenes Schüttgut zu erzeugen, wodurch es für Umschlags- und Verwertungsprozesse durch die dabei homogenisierten Eigenschaften eine Aufwertung erfährt. Für die Qualität von Holzhackschnitzeln werden Parameter im Bezug auf ihre äußere Form und ihre Inhaltsstoffe herangezogen. Diese sind in verschiedenen Standardisierungen, meist entsprechend der späteren Verwendung des Häckselgutes, festgelegt worden. Auf den industriellen Anforderungsbereich an die Qualität von Holzhackschnitzeln soll in diesem Fall nicht eingegangen werden, da der Ausgangsrohstoff Landschaftspflegeholz nur eine verminderte Eignung zur industriellen Nutzung zeigt (CREMER ET AL., 2007).

Ein weit verbreiteter Verwendungsbereich von Holzhackschnitzeln ist als Biomassebrennstoff zu finden. Holzhackschnitzel sind für diesen Anwendungsbereich in der europäischen Biobrennstoffnorm CEN/TS 151491, 2005 klassifiziert und deren Eigenschaften quantifiziert worden. Die Norm stellt somit die Basis für die Marktfähigkeit von Holzhackschnitzeln entsprechend der dort aufgeführten Qualitätsparameter Hackstückgrößenverteilung, Wassergehalt, Aschegehalt und Stickstoffgehalt. 
Informativ können dazu auch noch der Chlorgehalt, die Schüttdichte und der Heizwert herangezogen werden.

Grundsätzlich stellen auch die Maßverhältnisse der äußeren Form der Hackschnitzel selbst ein Qualitätskriterium dar, welches jedoch in der Normierung nicht erfasst wird, wie HASCHKE (2000) in seinen Untersuchungen feststellt. Dieses wird allgemein nicht über die üblichen Bestimmungsverfahren der Siebung in den gültigen Biobrennstoffnormen abgedeckt und führt bei der Bestimmung prinzipiell auch zu Abweichungen von den realen Abmaßen der Holzhackschnitzel. Auch SCHOLZ \& IDLER (2005) bestätigen dieses, da die äußere Aufarbeitungsgüte hinsichtlich Rauhigkeit und Zerfaserungsgrad einen Einfluss auf die Lagerstabilität von Holzhackschnitzeln aufweisen. Des Weiteren sei der Grad der zulässigen Sporenbelastung normativ nicht erfasst, obwohl dieser stark mit Abbauprozessen im Häckselgut in Verbindung steht, die eine Verwertbarkeit einschränken können. 


\section{Material und Methoden}

\subsection{Vorgehensweise in der Entwicklung}

Die Entwicklung des vollmechanisierten Häckslerkonzeptes zur Entbuschung von Offenflächen erfolgt in Rahmen eines zeitlich aufeinander folgenden Entwicklungsprozesses. Die einzelnen Phasen und Schritte bauen entsprechend ihrer Ergebnisse aufeinander auf. Vereinzelt treten Rückkopplungen aufgrund von Neubewertungen oder auch Entwicklungsschleifen (z.B. bei Überarbeitungen) auf. Die Arbeiten zur Produktentwicklung von ENGELN (2011) und von LINDEMANN (2005) stellen bei der Erstellung und Bearbeitung des Entwicklungsprozesses eine grundlegende Orientierungshilfe dar. Die dortigen Entwicklungsmuster sind jedoch, ähnlich wie auch bei PONN \& LINDEMANN (2008), stark an industriellen Betriebs- und Entwicklungsstrukturen einer Produktentwicklung ausgerichtet und beziehen z.B. auch das Controlling und das Marketing während des Gesamtprozesses ein. Da diese eher betriebliche Betrachtungsweise nicht Kern dieser Arbeit war, können die Prozessstrukturen auf die technisch/ technologische Entwicklung reduziert werden.

In Abb. 10 ist das Schema des hier durchgeführten Entwicklungsprozesses aufgezeigt. Es teilt sich in vier Phasen: in eine Vorbereitungs-, zwei Vorentwicklungs- und eine Entwicklungsphase. Letztlich schließt der Prozess mit einem Fazit zum entwickelten Häckslerkonzept, an welches sich entsprechend des Ergebnisses weiterführende Entwicklungsschritte oder Prozesse der Produktentwicklung anschließen könnten. Diese sind aber nicht Gegenstand dieser Arbeit. In industriellen Produktentwicklungsprozessen würden z.B. weitere Schritte zur Produktverifizierung, zur Serienfertigung und zur Produktimplementierung folgen. 


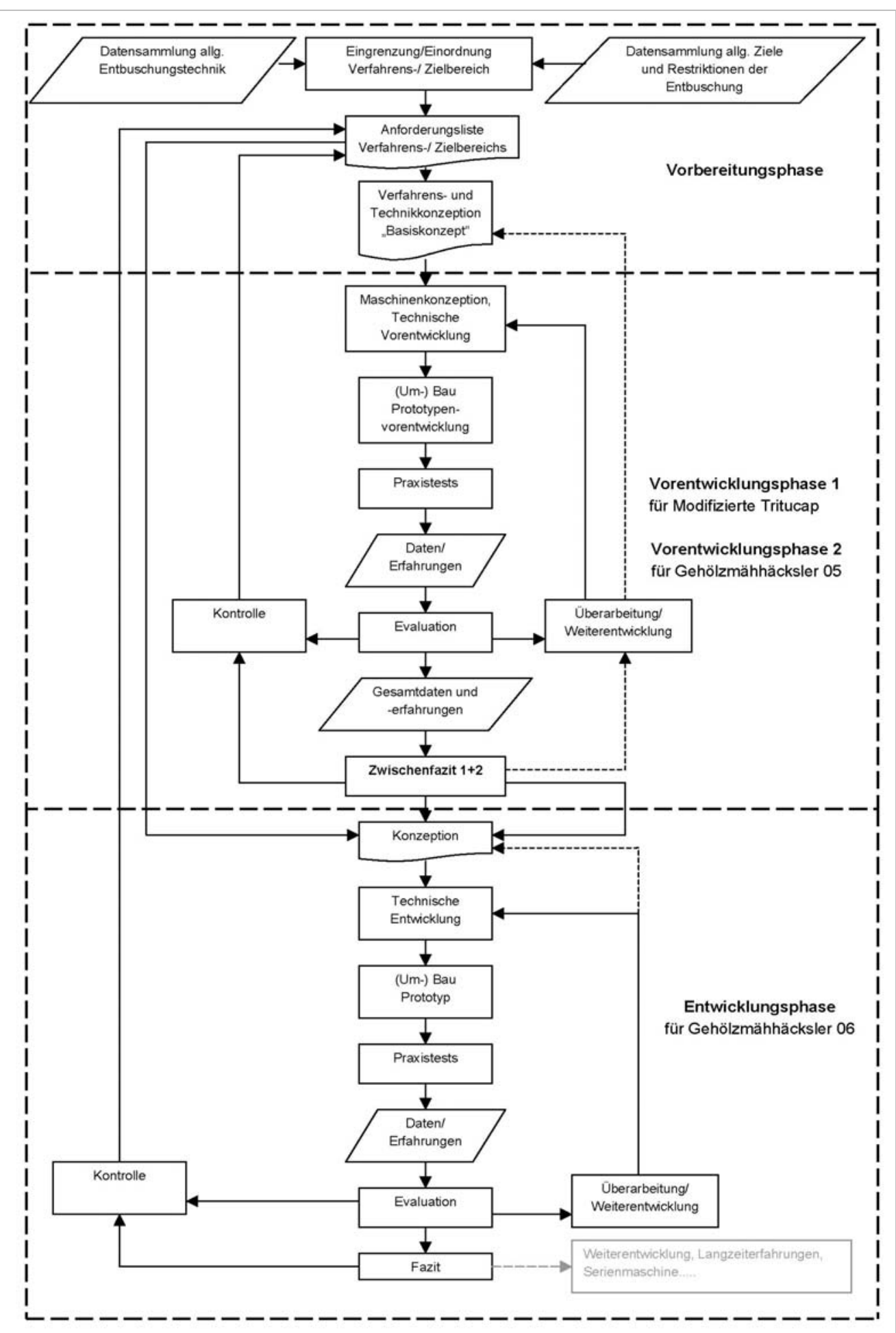

Abb. 10: Ablaufschema des Entwicklungsprozesses zur Konzeptentwicklung der vollmechanisierten Landschaftspflege

Die Vorbereitungsphase dient im weitesten Sinne der Verfahrensentwicklung, auf der die Entwicklung des Maschinenkonzeptes aufbaut. Zu Beginn wird der Verfahrens-/ Zielbereich der Entwicklung definiert, in dem anschließend 
die Anforderungsliste zur Entwicklung erstellt wird. Die Anforderungsliste beruht neben eigenen Überlegungen auf fachspezifischer Literatur zur Entbuschung von Offenflächen sowie den Aussagen von Akteuren der Landschaftspflege. In dieser Phase werden die verfahrenstechnischen und auch naturschutzfachlichen Anforderungen definiert, welche ein vollmechanisiertes Maschinenkonzept letztlich leisten muss. Anhand dieser wird ein Basiskonzept entwickelt. Das Basiskonzept stellt die Verfahrensausrichtung dar, in die technologische Lösungsansätze eingearbeitet werden. Diese werden anschließend in den Vorentwicklungsphasen in erste Maschinenkonzepte umgesetzt. Nach der praktischen Prüfung der Konzepte werden die gewonnen Ergebnisse anhand relevanter Punkte der Anforderungsliste in einer Evaluation diskutiert und in die weiteren Konzeptentwicklungsschritte integriert. Den Abschluss dieses Entwicklungsprozesses bildet die Funktionsprüfung und die Bewertung des entwickelten Maschinenkonzeptes einschließlich einer Betrachtung auf Verfahrensebene.

Da die Funktion einer derart komplexen Maschinenanwendung mit einer Vielzahl von Wechselwirkungen der Technik mit der Vegetation einhergeht, kann in dieser Arbeit zur Konzeptfindung kein Weg über ausschließlich quantifizierbare Ergebnisse gegangen werden.

Der Weg dieser Untersuchungen besteht deshalb vornehmlich darin, qualitative Aussagen zur Einsatzfähigkeit bzw. Eignung von technischen Komponenten und deren Zusammenwirken untereinander bei der Entbuschung zu ermitteln.

Zur qualitativen Beurteilung werden die Einsatzerfahrungen hinsichtlich Arbeitsverhalten, Bedienbarkeit, Art und Umfang von Störungen und Schäden im Bezug auf die bearbeitete Vegetation dokumentiert. Da die funktionelle Beurteilungen des Maschinenkonzeptes an vielfache Bedingungen geknüpft ist, deren Zusammenwirken sich messtechnisch auch aufgrund der Einsatzbedingungen nur schwer erfassen lässt, können deshalb im Rahmen des Entwicklungsprozesses vielfach nur beobachtete Wirkzusammenhänge unter Einbezug dabei aufgenommener Kennwerte 
einer objektiven Beurteilungen und Diskussion unterworfen werden. In Einzelfällen sind nähere messtechnische Untersuchung möglich, die jedoch aufgrund ihrer einmaligen Notwendigkeit nicht als Untersuchungsmuster im gesamten Entwicklungsprozess durchgeführt werden.

\subsubsection{Durchführung der Praxistests}

Bei der Durchführung der Praxistests werden gezielt verschiedenste Verbuschungsbedingungen auf den Flächen angefahren und bearbeitet. Dabei wird allgemein zwischen den Faktoren Stammform, verschiedenen Wurzelhalsdurchmessern (WHD) sowie verschiedenen Bestandesdichten und -breiten unterschieden. Eine Einteilung der Verbuschungen erfolgt in Klassen nach der folgenden Tabelle (Tab. 1). Dabei wird versucht Büsche und Bäume in einer größtmöglichen Kombination dieser Faktorklassen zu bearbeiten, um ein umfangreiches Bild von der Einsatzfähigkeit der eingesetzten Technik zu bekommen. Zudem wird in der allgemeinen Dokumentation sowie im Fortlauf dieser Arbeit diese Einteilung verwendet, um den Zustand der Verbuschung bei einzelnen Testphasen zu beschreiben. 
Tab. 1: Einteilungsklassen der Verbuschungen nach Stammform, Wurzelhalsdurchmesser, Bestandesdichte und Bestandesbreite mit ihren Ausprägungen

\begin{tabular}{|c|c|c|c|c|c|c|c|}
\hline $\begin{array}{l}\text { Stamm- } \\
\text { form }\end{array}$ & & $\begin{array}{l}\text { Wurzel- } \\
\text { hals- } \varnothing\end{array}$ & & $\begin{array}{l}\text { Bestandes- } \\
\text { dichte }\end{array}$ & & $\begin{array}{l}\text { Bestandes- } \\
\text { breite }\end{array}$ & \\
\hline Klasse & $\begin{array}{c}\text { Ausprägung } \\
\text { Gehölz }\end{array}$ & Klasse & $\begin{array}{c}\text { Ausprägung } \\
\text { Gehölz }\end{array}$ & Klasse & $\begin{array}{c}\text { Ausprägung } \\
\text { zw. Gehölzen }\end{array}$ & Klasse & $\begin{array}{c}\text { Ausprägung } \\
\text { Bestand }\end{array}$ \\
\hline baumartig & $\begin{array}{c}\text { ein } \\
\text { ausgeprägter } \\
\text { Trieb/ } \\
\text { Wurzelstock }\end{array}$ & $\begin{array}{l}\text { sehr } \\
\text { dünn }\end{array}$ & bis $2 \mathrm{~cm}$ & freistehend & Abstand >Höhe & klein & $\begin{array}{c}\text { <Bearbeitungs } \\
\text {-breite }\end{array}$ \\
\hline \multirow[t]{4}{*}{ buschartig } & $\begin{array}{c}\text { mehrere } \\
\text { Triebe/ } \\
\text { Wurzelstock, } \\
\text { ab Boden }\end{array}$ & dünn & $>2-6 \mathrm{~cm}$ & engstehend & $\begin{array}{c}\text { Abstand < Höhe } \\
\text { und nicht } \\
\text { verwachsen }\end{array}$ & $\begin{array}{l}\text { einseitig } \\
\text { geschlossen }\end{array}$ & $\begin{array}{l}\text { >Bearbeitungs } \\
\text {-breite, jedoch } \\
\text { einseitig } \\
\text { Bestandes- } \\
\text { rand }\end{array}$ \\
\hline & & mittel & $>6-8 \mathrm{~cm}$ & verwachsen & $\begin{array}{c}\text { Äste/ Triebe } \\
\text { überschneiden } \\
\text { sich }\end{array}$ & geschlossen & $\begin{array}{l}\text { >Bearbeitungs } \\
\text {-breite }\end{array}$ \\
\hline & & dick & $>8-12 \mathrm{~cm}$ & & & & \\
\hline & & sehr dick & $>12 \mathrm{~cm}$ & & & & \\
\hline
\end{tabular}

Direkte Auswirkungen des Einsatzes auf Flora und Fauna sowie auf den Boden werden je nach erkennbarem Auftreten dokumentiert und nach den Einsätzen vorerst visuell aufgenommen.

Für erste wissenschaftliche Untersuchung der Einsatzauswirkungen werden bei den Praxiseinsätzen den beteiligten Projektpartnern entsprechende Parzellen von Verbuschungen frei gestellt. Die im Projektzeitraum ermittelten Ergebnisse werden durch einen ständigen Erfahrungsaustausch übermittelt und können so schon in die Evaluation bzw. Entwicklung der Maschinentechnik einfließen. Die Ergebnisse werden auch zu abschließenden Bewertung des entwickelten Verfahrenskonzeptes herangezogen. 


\subsection{Versuchsflächen}

Zur Durchführung der Praxisversuche werden dem Projekt im Raum Göttingen (Niedersachsen) mehrere Versuchsflächen zur Verfügung gestellt. Das Gebiet ist Teil des Leine- Weser- Berglandes (Niedersächsischen Bergland) in den deutschen Mittelgebirgsgebieten Südniedersachsens. Die Versuchsflächen liegen fast ausschließlich im FFH- Gebiet "Göttinger Wald“ (EU- Kennzahl 4325-301; landesinterne Nummer 138) und sind vielseitig durch Verbuschung gekennzeichnet. Geologisch sind sie dem Oberen Muschelkalk zuzuordnen und der Boden besteht aus steinig- tonigem Kalkstein- Verwitterungsboden. Die mittlere Jahrestemperatur beträgt in diesem Gebiet $7,5-8^{\circ} \mathrm{C}$, die Höhe ca. $330 \mathrm{~m}$ ü. NN. und die Jahresniederschlagsmenge $650-700 \mathrm{~mm}$. Lediglich eine verbuschte Kompensationsfläche aus Ausgleichs- und Ersatzmaßnahmen, welche für Versuche genutzt wird, liegt in direkter Randlage des FFH- Gebietes.

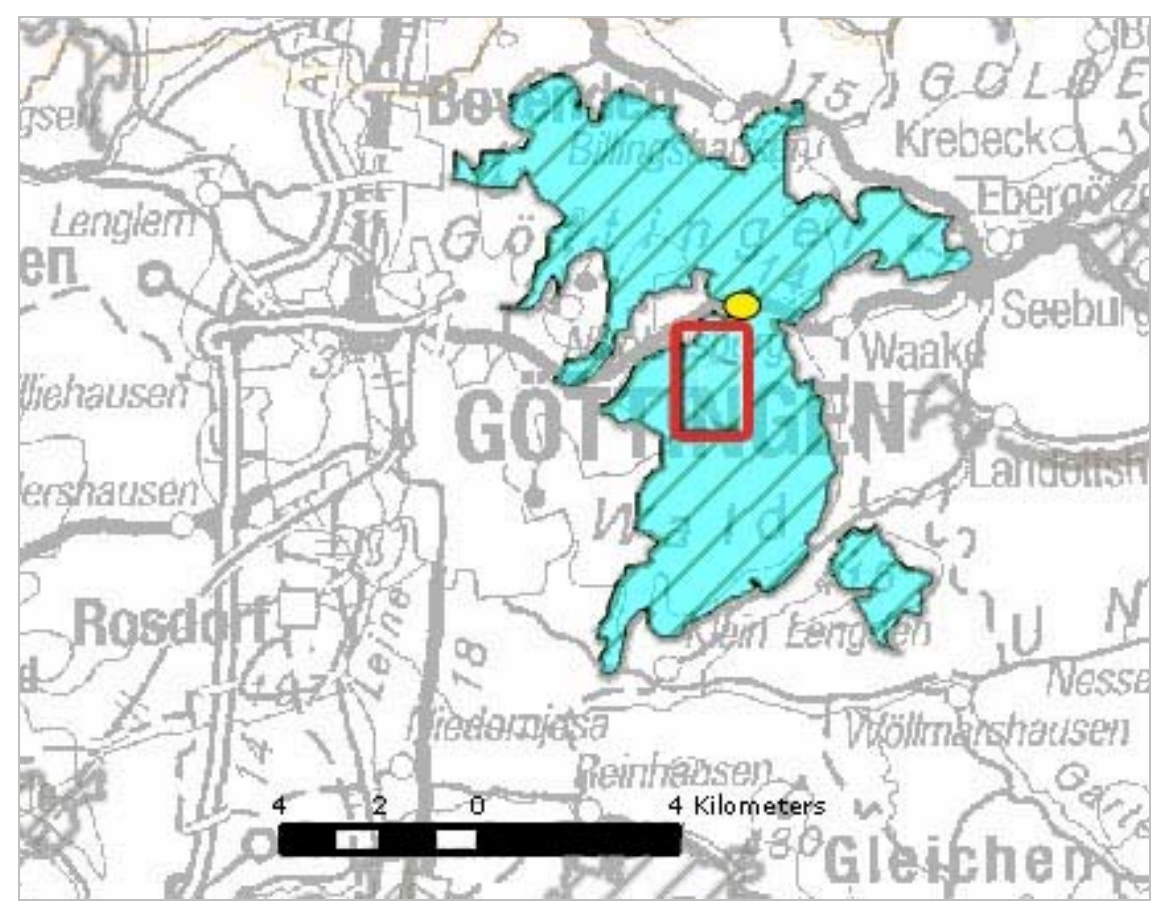

Abb. 11: Lage der Versuchsflächen im FFH- Gebiet „Göttinger Wald“; gelb = Ausgleichsfläche Roringen (AFR); rot umrandet = Kartenausschnitt Abb. 12 


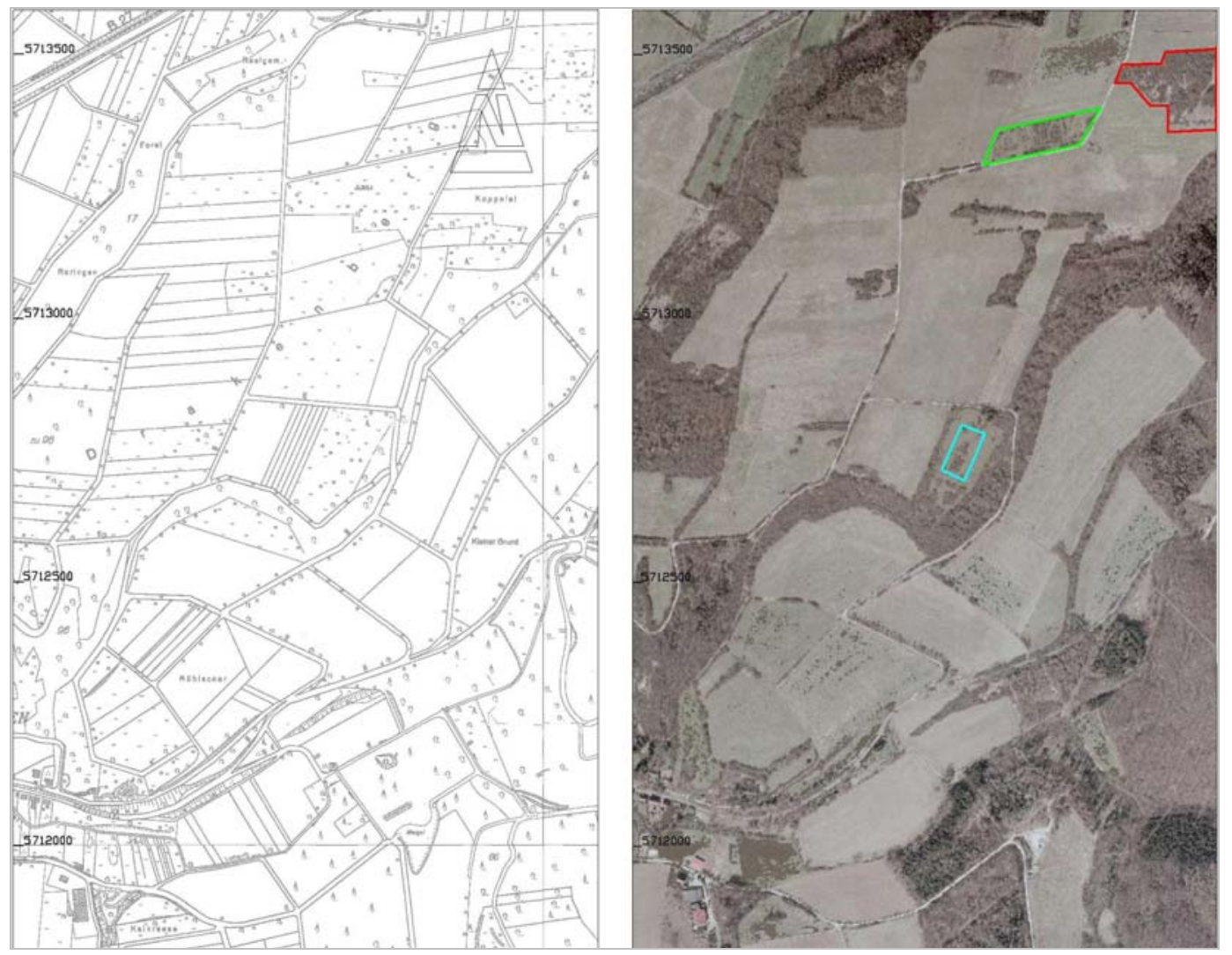

Abb. 12: Karten- und Luftbildausschnitt zur Lage der Versuchsflächen auf dem Drakenberg; rot = Drakenberg 1 (DB1); grün = Drakenberg 2 (DB2); blau = Drakenberg 3 (DB3); Maßstab: 1:13000 (MEINEKE, 2006; verändert)

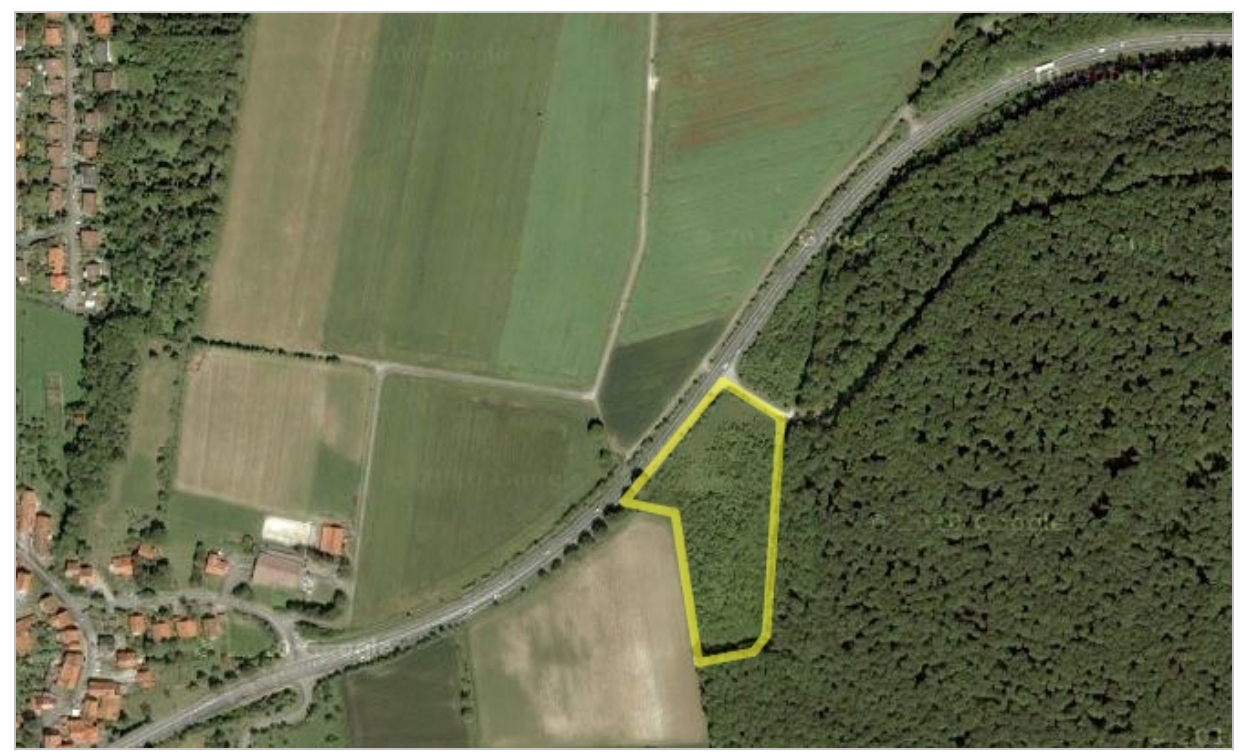

Abb. 13: Lage der Versuchsfläche „Ausgleichsfläche Roringen“ (AFR, gelb) im Südosten von Roringen bei Göttingen; Maßstab 1:10000; (Quelle: GoogLEMAPS, 2011) 


\subsubsection{Drakenberg 1 (DB1)}

Die Versuchsfläche DB1 (in Abb. 12 rot umrandet) ist eine zusammenhängende ca. 1,5 ha große Sukzessionsfläche, welche auf einem 4 ha Grünlandschlag integriert ist. Die weitflächigen unverbuschten Teile des Schlages werden jährlich zur Heugewinnung geschnitten und als Umtriebsweide genutzt. Über den Werdegang der verbuschten Teile auf der Sukzessionsfläche können leider keine genauen Informationen ermittelt werden. Sie scheinen aber mehrere Jahre oder gar schon Jahrzehnte nicht mehr einer Bewirtschaftung zu unterliegen. Die Fläche ist in südlicher Richtung gleichmäßig zu ungefähr $4 \%$ geneigt und bis auf wenige Senken relativ eben. Lokal sind kleinere Absätze von ca. 10- $15 \mathrm{~cm}$ Höhe auf einen Meter bis wenige Meter vorzufinden, die vermutlich auf Grenzen der Bewirtschaftungsart hindeuten (alte Pflugfurchen oder ehemalige Zäunungen).

Die Gehölzvegetation besteht vornehmlich aus Weiß- und Schwarzdornbüschen (geschätzt ca. 90\%), welche stellenweise auch mit Hartriegel und Hundsrose durchsetzt sind. Platzweise sind Eschen etabliert vorzufinden, die in Gruppen verschiedener WHD- Stärken zusammenstehen. Auffällig ist, dass sich am nördlichen Rand ein breiter Streifen von ca. $10 \mathrm{~m}$ und ab der Hälfte von ca. $20 \mathrm{~m}$ Breite von Ost nach West zieht, in welchem die Gehölzvegetation durchweg sehr stark entwickelt ist. Vereinzelt gemessene Wurzelhalsdurchmesser (WHD) reichen bis zu $20 \mathrm{~cm}$ und die Höhe bis zu ca. $6 \mathrm{~m}$, wobei einzelne Eschen noch mehrere Meter darüber hinausragen. Es wird vermutet, dass es sich um einen älteren Heckenstreifen handelt, von dem sich die Gehölzvegetation südwärts ausbreitete. Die augenscheinliche Stärke und Höhe der Gehölze nimmt in südlicher Richtung insgesamt ab und wird von einem geschlossenem Bestand immer lückiger, bis eine eher nesterweise Verteilung der Gehölze vorliegt. Teilweise sind gehölzfreie Streifen und Plätze von bis zu $15 \mathrm{~m}$ Durchmesser erhalten geblieben. In diesem augenscheinlich jüngeren Teil der Verbuschung, der fast ausschließlich aus Weiß- und Schwarzdorn besteht, können immer wieder einzelne stark entwickelte Exemplare ausgemacht werden. Während 
der überwiegende Teil WHD bis $6 \mathrm{~cm}$ erreicht und ca. 2,5- $3 \mathrm{~m}$ hoch ist, sind einzelne mit 15 bis zu $20 \mathrm{~cm}$ WHD vertreten. Dabei treten auch immer wieder stark entwickelte Stöcke auf, die einen Gesamtdurchmesser von $30 \mathrm{~cm}$ erreichen, aber schon an der Bodenoberfläche aus bis zu 15 Einzeltrieben mit bis zu $8 \mathrm{~cm}$ Durchmesser bestehen. Bei den stark entwickelten Exemplaren in diesem Bereich handelt es sich wahrscheinlich um früher schon solitär stehende Büsche, bevor die Bewirtschaftung der Fläche aufgegeben wurde.

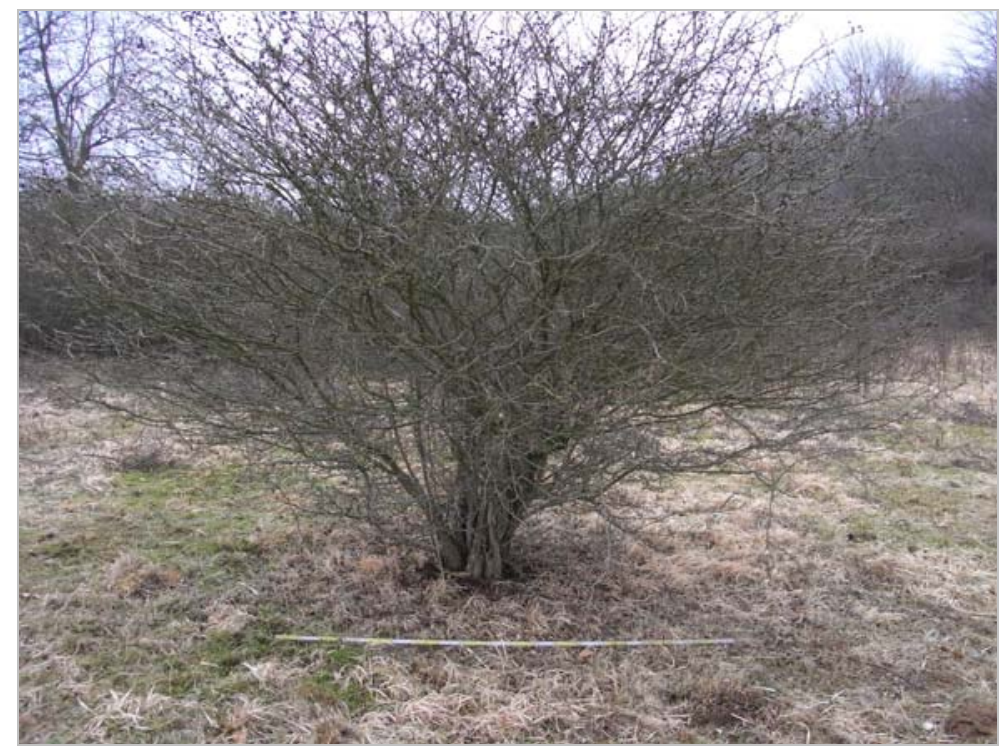

Abb. 14: Stark entwickelter Weißdornbusch (sehr dick, freistehend) auf der Versuchsfläche Drakenberg1 (DB1); Verzweigung des Stocks direkt über der Bodenoberfläche zu 11 Trieben (Zweimeter- Maß im Vordergrund liegend)

Der südliche Teil ist wiederum von stärkeren, anscheinend älteren Pflanzen geprägt. In diesem Bereich sind mehrere große Lesehaufen von Steinen vorzufinden, welche zur frühen Gehölzbesiedelung gedient haben könnten.

Vor allem im mittleren Bereich weißt die Fläche von Ost nach West Gehölzstrukturen auf, in der sich buschartige Gehölze von sehr dünn bis sehr dick und in allen Bestandesdichten bearbeiten lassen. Aussagen zur Bearbeitbarkeit baumartiger Gehölze sind auf dieser Fläche aufgrund des geringen Vorkommens nicht zu erwarten. 


\subsubsection{Drakenberg 2 (DB2)}

Eine weitere Versuchsfläche befindet sich in direkter Nähe südöstlich von DB1. Die Fläche DB2 ist in Abb. 12 grün umrandet dargestellt. Sie hat eine Größe von 1,1 ha und steht in Form einer Umtriebsweide mit Robustrindern (Scotish Highland Cattle, Galloway) in Bewirtschaftung. Die Fläche ist durchweg eben und hat keinerlei Gefälle. Der westliche Teil ist fast vollständig mit stark entwickelten und offenbar schon sehr alten Weiß- und Schwarzdornbüschen verbuscht. Probemessungen ergeben WHD von bis zu $18 \mathrm{~cm}$, wobei sich die Gehölze teilweise schon direkt über dem Boden in mehrere dünnere Triebe von 5-7 cm aufteilen. Bei einer Höhe von 2,5- 3,5 m erreichen sie auch annähernd diese Werte beim Kronendurchmesser. Vermutlich durch die ständig wiederkehrende Beweidung stehen sie aber relativ licht, so dass sich ihre Kronenäste meist gerade nur berühren. Junger Unterbewuchs fehlt oder ist nur verkümmert festzustellen. Im zweiten Drittel geht die Fläche Richtung Osten in einen weit lichteren Bestand über, der durch einzeln stehende Büsche oder Gebüschgruppen mit vornehmlich 2- 5 Stöcken geprägt ist. In diesem Bereich sind zu ca. 50\% auch Hundsrosen (Rosa canina) vertreten. Diese sind teilweise sehr stark entwickelt und stehen solitär mit bis zu 30 Trieben pro Stock, wobei Ihr Kronendurchmesser bei einer Höhe von 3 m durchaus über $4 \mathrm{~m}$ erreichen kann. Die Gehölze sind insgesamt von sehr dünn bis sehr dick ausgeprägt. Bis auf wenige Eschen sind hier nur buschartige Gehölze vorzufinden.

\subsubsection{Drakenberg 3 (DB3)}

Die Versuchsfläche DB3 ist ebenfalls in der Nähe der vorher beschriebenen Versuchsflächen auf dem Drakenberg (blau in Abb. 12). Sie besteht aus einem Gehölzstreifen, der sich mit einer ungefähren Breite von $15 \mathrm{~m}$ und einer ungefähren Länge von 100 m als „Gehölzinsel“ in einer Grünlandfläche von 1,3 ha befindet. Die Gesamtfläche wurde bis in die 1960er Jahre ackerbaulich genutzt und verbrachte anschließend. Die Fläche um den Gehölzstreifen wurde 1995 bzw. 1997 im Rahmen von Beweidungsversuchen vollständig entbuscht (RAHMANN, 2000). Das Alter der Gehölze wird auf bis zu 40 Jahre geschätzt. Der Gehölzbestand aus 
Weißdorn, Hartriegel und Hundsrose war aus diesem Grund insgesamt sehr stark entwickelt und der Bestand ist vollständig geschlossen. Innerhalb der Sukzessionsfläche dominiert der Weißdorn, wobei Hartriegel und Rose mit wenigen Exemplaren (<10\%) eher im Randbereich vertreten sind. Ca. 50\% der Triebe erreichen einen WHD über $6 \mathrm{~cm}$. Vor allem in der Fläche ist der Anteil mit Trieben $>12 \mathrm{~cm}$ WHD recht groß. Im Abstand von 2- $3 \mathrm{~m}$ kann mindestens ein Gehölz mit einem „sehr dicken“ WHD festgestellt werden. Die Höhe des Bestandes erreicht ca. $6 \mathrm{~m}$. Einige Eschen sind im Bestand vorzufinden, welche aber mit einem WHD von über $25 \mathrm{~cm}$ und einer Höhe von über $10 \mathrm{~m}$ schon als etablierte Bäume gelten können. Bis auf wenige Ausnahmen am Rand der Sukzessionsfläche ist kein weiterer Eschenaufwuchs vertreten.

\subsubsection{Ausgleichsfläche Roringen (AFR)}

Am östlichen Ausgang der Bundestrasse 27 von Roringen besteht die vierte Versuchsfläche aus einer annähernd 2 ha großen Ausgleichsfläche, welche 1995 als Kompensationsmaßnahme in Form einer Streuobstwiese angelegt wurde. Ursprünglich handelt es sich um Ackerland. Im Nordosten, Osten und Süden grenzt die Fläche an einen Buchen- Eschen- Hochwald. Die Fläche ist sehr eben und hat ein unwesentliches Gefälle in nördlicher Richtung.

Aufgrund stark vernachlässigter Pflegemaßnahmen ist die Ausgleichsfläche (in Abb. 11 und Abb. 13 gelb markiert) innerhalb von zehn Jahren nach der Anlage zu ca. dreiviertel verbuscht. Lediglich ein Streifen von ca. 20 m Breite im nördlichen Bereich parallel zur Bundesstrasse (Nordost- SüdwestAusrichtung) ist annähernd gehölzfrei. Auf der Fläche sind Eschen, Weiden, Hartriegel, Birken und einige wenige Weißdorn- sowie Rosenbüsche vertreten. Stark dominierend ist die Esche (ca. 85\%), gefolgt von Birke und Hartriegel.

In einem schmalen Übergangsbereich von ca. $25 \mathrm{~m}$ parallel zur gehölzfreien Fläche schließt sich der Bestand zunehmend in östliche und südliche Richtung. Hier sind von aufkeimenden Eschen und Birken auch Durchmesser bis zu einem WHD von $8 \mathrm{~cm}$ vorzufinden. Entsprechend reichen die Höhen 
der Gehölze von unter einem Meter bis zu $7 \mathrm{~m}$. Dabei gibt es sehr lichte Bereiche mit einigen Metern Platz zwischen Einzeltrieben oder Gruppen von Trieben und dichtere mit mehreren meist dünnen $(<3 \mathrm{~cm}$ WHD) pro Quadratmeter. In diesem Bereich sind auch die wenigen Weißdorn- und Rosenbüsche vorzufinden, deren Höhe aber kleiner als 2-2,5 m ist. Der WHD der Weißdornbüsche liegt dabei nicht über $5 \mathrm{~cm}$.

In der nördlichen Hälfte der Fläche am östlichen Waldrand ist ein Streifen von 10- $20 \mathrm{~m}$ und in der südlichen Hälfte annähernd die gesamte Fläche derart verbuscht, so dass es nur schwer möglich ist, hindurchzugehen. Stellenweise stehen bis zu 9 Triebe pro Quadratmeter. Probemessungen ergeben in diesem Bereich eine mittlere Triebzahl von 4,2 Trieben pro Quadratmeter. Dabei reicht die WHD- Spanne von 3-13 cm. Wobei ca. 50\% der Triebe einen Durchmesser von 4- $7 \mathrm{~cm}$ haben. Die Triebe erreichen hier ein Höhe bis zu 9 m.

Von den angepflanzten Obstgehölzen sind nur noch auf der Freifläche und der Übergangsfläche nennenswerte Vorkommen erhalten geblieben. Einige Walnussbäume und Kirschen, die frei stehen, haben sich gut entwickelt. Umso stärker und offensichtlich umso länger sie in der dichten Fläche bedrängt worden sind, umso kümmerlicher oder gertenförmiger ist ihr Wuchs.

\subsection{Häckslerkonzept „Tritucap 2“}

Eine mögliche Basistechnik der Entwicklungen kann die Tritucap 2 darstellen, ein selbstschneidenden Schneckenhäcksler, welcher von BLOCK (2004) zur nichtbrennenden Flächenvorbereitungen in Amazonien eingesetzt wurde (Abb. 15; Abb. 16). 


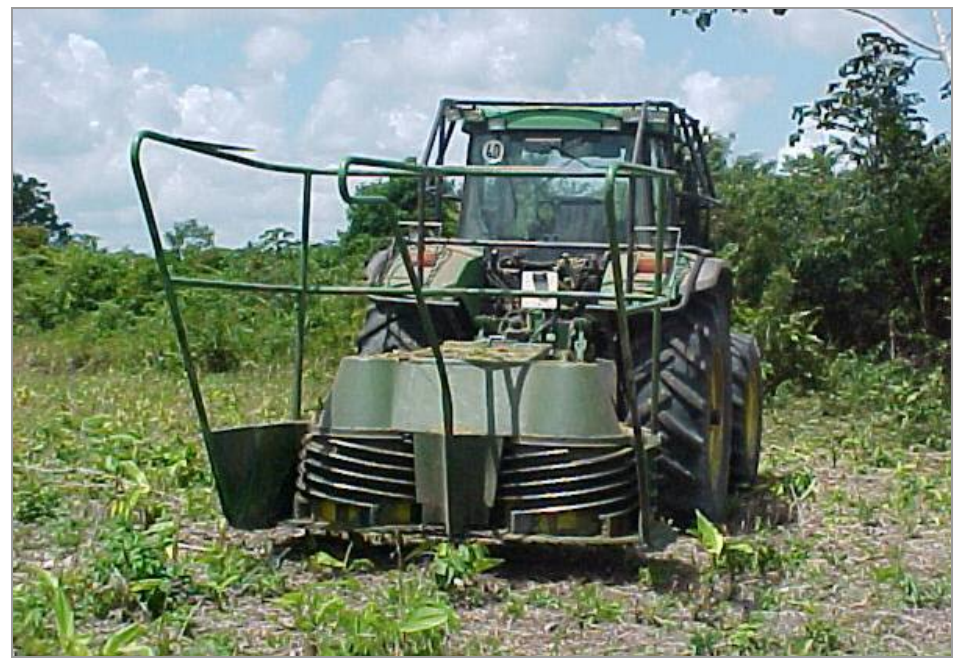

Abb. 15: Mähhäcksler „Tritucap 2“ am Einsatzort in Amazonien (BLOCK, 2004)

Die Maschine war zur Zerkleinerung von unstrukturiert gewachsener Sekundärwaldvegetation entwickelt worden. Diese wird in einem Arbeitsgang geschnitten, zerhäckselt und als Mulchdecke über die Fläche verteilt. Eine Materialbergung erfolgt nicht. Die Maschine wird im heckseitigen Schlepperanbau in der Dreipunkt-Hydraulik eines landwirtschaftlichen Schleppers in Schubfahrt betrieben und arbeitet reihenunabhängig über die Schlepperbreite. Die Tritucap zerkleinert das Material mit zwei senkrecht stehenden, konischen Häckselrotoren, die jeweils mit vier geschärften Schneiden bestückt sind, welche sich schneckenartig um den Rotorkörper ziehen (sogenannter „viergängiger Häckselrotor“). Der Windungsabstand beträgt $80 \mathrm{~mm}$ und die Windungstiefe $100 \mathrm{~mm}$. Waagerecht unterhalb der Rotoren ist jeweils ein Sägeblatt von $992 \mathrm{~mm}$ Durchmesser angebracht, welches das Material bodennah abtrennen kann. Bei dieser Sonderbauform eines Schneckenhackers wird das Material nicht axial von der Spitze aus auf den Konusrotor geführt, sondern tangential an der Basis (siehe auch Abb. 27). Durch die Rotordrehung wird das Material zu dem jeweiligen Häckselspalt des Rotors an den Außenseiten der Maschine transportiert (tangentialer Transport mit dem Rotorumfang), wo es zerkleinert wird. Idealerweise geschieht dieses ohne ein Umfallen des Materials, also bei senkrecht stehenden Trieben. Der Häckselspalt ist keilförmig ausgestaltet und fungiert als Widerlager zu der tangentialen Materialförderbewegung des 
Rotors, so dass das Material in dem Häckselspalt fixiert und eingeklemmt wird. Die Schneiden schneiden dort in das Material ein und durch die abwärts gerichtete Schraubbewegung findet eine axial zur Rotorachse gerichtete Einzugsbewegung statt (senkrechter Einzug der im Häckselspalt stehenden Triebe). Bei diesem Vorgang schneiden die Schneiden durch die Verjüngung des Häckselspaltes fortwährend tiefer in das Material und brechen dabei zwischen den Schneiden Hackstücke heraus. Das zerkleinerte Material wird durch Auswerfer, welche zwischen Sägeblatt und Rotorkörper angeordnet sind, mit hoher Geschwindigkeit zum Auswurfschacht befördert. Die Auswerfer stehen $2 \mathrm{~cm}$ über das Äußere der Schneckenwindung über und sind jeweils am Ende einer Schneckenwindung angebracht. Durch die Positionierung der Auswerfer zwischen Sägeblatt und Häckselschnecke ist es unabdingbar, dass ein gewisser Teil des Stammquerschnitts beim Einklemmen sowie auch beim axialen Einzug durch die Auswerfer zerkleinert wird.

Der Antrieb der Häckselrotoren erfolgt über die Schlepperzapfwelle und wird über ein zentrales T- Getriebe auf beide Häckselrotoren geleitet. Zwischen den Rotoren und dem T-Getriebe sind jeweils Nockenschaltkupplungen mit einem Lösemoment von $2500 \mathrm{Nm}$ angebracht. Diese sind jedoch entgegen der Drehrichtung montiert, um bei Stillstand eines Häckselrotors den Antriebsstrang vor der kinetischen Energie des anderen zu schützen, welcher mit hohem Drehmoment auf den stehenden Häckselrotor wirken würde (siehe BLOCK, 2004). 


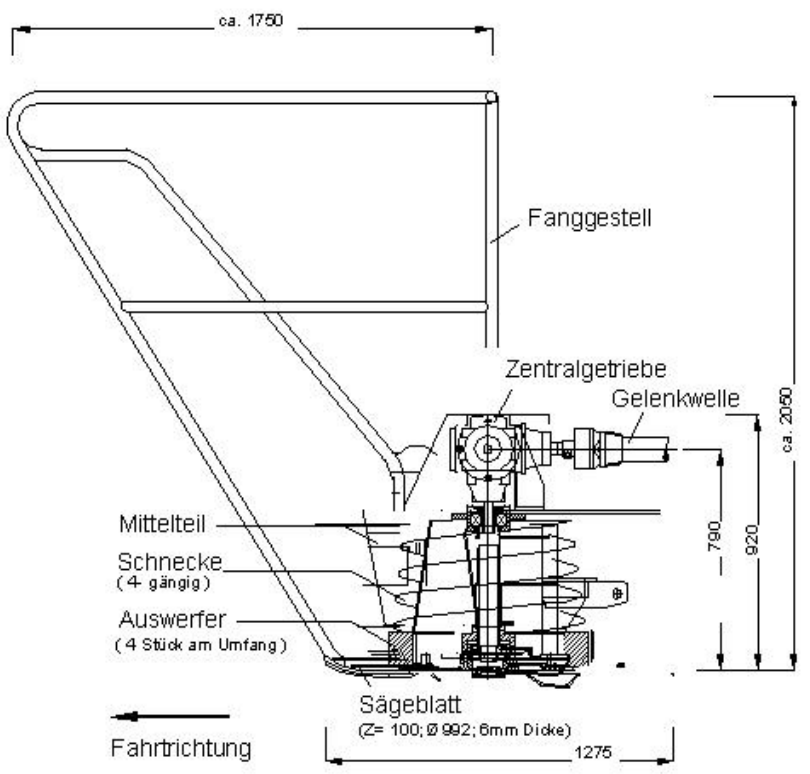

Seite

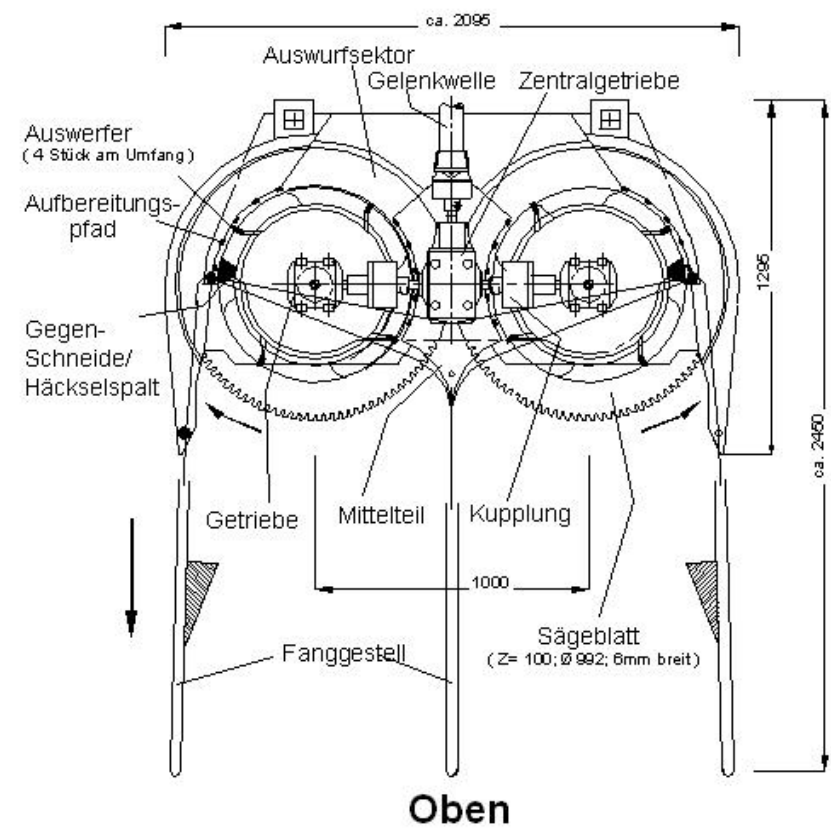

Abb. 16: Aufbau der Tritucap in der Grundform ohne Überladeeinrichtung (skizziert).

Der gesamte Antriebsstrang ist zwischen Schlepper und Maschine mit einer Reibkupplung (3000 Nm Lösemoment), welche im Zapfwellengelenk integriert ist, abgesichert. Die Übersetzung der Getriebe ist $1: 1$, so dass die Häckselrotordrehzahl der Drehzahl des Zapfwellenanschlusses des 
Schleppers entspricht. Bei einer Zapfwellendrehzahl von 540U/min (1000 U/min) erreicht die Umfangsgeschwindigkeit an der größten Ausdehnung des Häckselrotors $(750 \mathrm{~mm}$ Durchmesser an der Schneidenspitze) Werte von $21,21 \mathrm{~m} / \mathrm{s}(39,27 \mathrm{~m} / \mathrm{s})$ und die der Sägeblätter Werte von 28,05 m/ s $(51,94 \mathrm{~m} / \mathrm{s})$. Die viergängigen Häckselrotoren besitzen eine Gesamtsteigung von $320 \mathrm{~mm}$ (vier Schneidengänge $\times 80 \mathrm{~mm}$ Windungsabstand) und erreichen bei der Zerkleinerung eine axiale Einzuggeschwindigkeit des Holzes zum Rotor von rechnerisch 2,88 m/s $(5,33 \mathrm{~m} / \mathrm{s})$. Die relativ hohe Einzugsgeschwindigkeit ist der begrenzten Triebzahl geschuldet, welche von dem System bei dieser Art der Zuführung zeitgleich verarbeitet werden kann. So können in dem Häckselspalt je nach Durchmesser nur wenige bis ein Trieb fixiert und zerkleinert werden, bevor der oder die nächsten zur Zerkleinerung folgen können. Entsprechend wird die mögliche Arbeitsgeschwindigkeit durch die zu bearbeitende Trieblänge (bestehend aus Länge und Anzahl der Triebe) pro Fahrtmeter bestimmt.

Vor der Maschine ist ein so genanntes Fanggestell angebracht, welches das zu bearbeitende Material ausrichten und jeweils auf die Häckselrotoren separieren soll. Zudem soll es verhindern, dass vom Stock getrennte Triebe seitlich umfallen und somit den weiteren Gutfluss behindern oder gar auf das Antriebsfahrzeug kippen und dieses beschädigen.

\subsection{Antriebsmaschine}

Als Antriebsmaschine und Trägerfahrzeug für die Prototypen wird ein landwirtschaftlicher Allradschlepper John Deere 6920S mit einer Motorleistung von $118 \mathrm{~kW}$ verwendet, welcher mit einem Frontlader mit hydraulischer Werkzeugbetätigung ausgerüstet ist. Der Schlepper hat ein stufenloses Getriebe, mit dem ohne Kupplungsbetätigung die Fahrgeschwindigkeit während der Fahrt verändert werden kann. Eine schnelle und ruckfreie Geschwindigkeitsanpassung ist auf diese Art während der Arbeitsphasen möglich. Zum Schutz vor den verbleibenden Gehölzstümpfen und vor Dornen ist der Schlepper mit verstärkten Forstreifen mit einem Standard- AS- Profil ausrüstet (Reifengrößen hinten: 650/65R38, vorne: 540/65R28). Neben drei Zapfwellendrehzahlen (540, 750 und 
1000U/min) stehen vier doppeltwirkende Hydrauliksteuergeräte zur Verfügung, welche in ihrer Hydraulikölabgabemenge einstellbar sind. Das Leergewicht des Schleppers beträgt inklusive Frontlader $7100 \mathrm{~kg}$. Das Fahrzeug ist $2,42 \mathrm{~m}$ breit und ohne den Überstand des Frontladers gemessen 5,4 m lang. Der Radstand beträgt 2,65 m und die Koppelpunkte der Unterlenkeraufnahmen sind ungefähr 1,1 m von der Achse entfernt.

\subsection{Hackschnitzelqualität}

Die Hackschnitzelqualität der produzierten Hackschnitzel werden anhand der Parameter Korngröße und der Korngrößenverteilung untersucht. Die Korngrößenanalysen werden jeweils zum Ende der Entwicklungsphase des GMH 05 sowie des GMH 06 anhand des Materials der letzten Einsatztests durchgeführt. Die Ergebnisse sollen einen Hinweis geben inwieweit das Zerkleinerungsprinzip im nächsten Entwicklungsschritt hinsichtlich der Aufarbeitungsqualität überarbeitet werden muss.

Zudem wird zum Ende der Entwicklung an dem abschließenden Prototyp die Aufarbeitungsqualität des Zerkleinerungsaggregates noch einer genaueren Untersuchung unterzogen. Dabei werden die jeweiligen zwei Stammformtypen „baum- und buschartig“ in jeweils zwei verschiedenen Wurzelhalsdurchmesserklassen separat zerkleinert und die Korngrößen des Häckselmaterials nach dem Rüttelsiebverfahren bestimmt. Als typische Referenzpflanzen dienen die Esche für die baumartige und der Weißdorn für die buschartige Wuchsform.

Messungen zum Wassergehalt erfolgen im Rahmen der Durchführung der Korngrößenanalyse, da eine dortige Kontrolle des Wassergehaltes notwendig ist. Die Messung des Wassergehaltes zum Erntezeitpunkt wird aufgenommen, um eine Einschätzung zum Erntewassergehalt zu erhalten und diesen hinsichtlich der Lagerungsanforderungen einordnen zu können.

\section{Probenherstellung}

Die Probennahme erfolgt nach CEN/TS 14778-1 (CEN/TS 14778-1, 2004). Dazu wurden zum Ende jeder der drei Prototypentwicklungsphasen stichprobenartig 11 Einzelproben aus dem auf einem landwirtschaftlichen 
Anhänger gesammelten Häckselmaterial (ca. 6 Kubikmeter) entnommen. Dabei erfolgt die Probennahme über drei Höhenschichten der Schüttung verteilt und es wird mit den Einzelproben je Untersuchung ein Probenvolumen von ca. 65 Litern gewonnen. Die Einzelproben werden wieder miteinander zu einer Gesamtprobe vereinigt.

Die weiteren Proben zur genaueren Untersuchung der Aufarbeitungsqualität des GMH 06 werden separat gewonnen. Dazu werden händisch Eschenund Weißdorntriebe auf den Versuchsflächen bodennah abgeschnitten und diese vermessen. Die Triebe werden jeweils nach Esche und nach Weißdorn in zwei Wurzelhalsdurchmesserklassen mit einem Wurzelhalsdurchmesser (WHD) von 1- $6 \mathrm{~cm}$ und einem WHD von 6- $11 \mathrm{~cm}$ sortiert. Anschließend werden die vier Sortierungen der Triebe den Zuführaggregaten des GMH 06 zugeführt und diese zerkleinert. Das Häckselmaterial der Sortierungen wird separat aufgefangen. Je Sortierung wird Probenmaterial von ca. 200 Liter produziert, aus dem wiederum stichprobenartig eine jeweilige Gesamtprobe mit einem Volumen von ca. 65 Litern gewonnen wird.

Allen Gesamtproben wird zur Wassergehaltsbestimmung des Frischmaterials Häckselmaterial von ca. 5 Litern entnommen. Das Probenmaterial zur Wassergehaltsbestimmung wird zur Durchführung von drei Wiederholungen in drei ungefähr gleich große Volumenteile aufgeteilt und in luftdichte Kunststoffbeutel verschlossen. Die restlichen ca. 60 Liter jeder Gesamtprobe werden für ca. vier Wochen in einem lufttrockenen Raum zur Trocknung ausgebreitet und mehrfach vorsichtig gewendet, um das erntefrische Material in eine Gleichgewichtsfeuchte mit der Umgebung zu bringen.

Nach der Lagerung der Restprobe wird sie nochmals zu einer Mischprobe gemäß CEN/TS 14780 aufbereitet (CEN/TS 14780, 2004), um eventuellen Entmischungsprozessen während der Lagerungsbehandlung entgegen zu wirken. Dazu werden die Einzelproben zu einem Kegel aufgeschüttet und dieser durch dreimal schichtweises Abtragen und wieder Aufschütten durchmischt. Anschließend wird die Gesamtprobe nach dem Mischkreuzverfahren auf ein Volumen von je ca. 30 Litern geteilt. Die eine Hälfte dient zur Bestimmung der Korngrößenanalyse und die andere Hälfte 
nach erneuter Reduktion auf ca. 5 Liter Probenvolumen der Wassergehaltsbestimmung zum Siebzeitpunkt. Auch diese 5 Liter Probenmaterial werden wiederum auf drei Proben aufgeteilt und in Kunststoffbeutel verpackt.

\section{Wassergehalt}

Der Wassergehalt wird nach der sogenannten Darrmethode oder auch Trockenschrankmethode der CEN/TS $14774-2$ bei $105^{\circ} \mathrm{C} \quad\left(+/-2^{\circ} \mathrm{C}\right)$ Trockenschranktemperatur bis zur Einstellung der Gewichtskonstanz ermittelt.

Die drei Probenbeutel werden einzeln auf einer Analysenwaage auf 0,1g genau gewogen und das Probenmaterial eines Beutels jeweils auf ein korrosions- und hitzebeständiges Edelstahlblech verteilt. Anschließend wird die Masse des Beutels nach Beseitigung von eventuellem Feuchtigkeitsniederschlag von der Probenmasse subtrahiert. Nach einer 48- stündigen Trocknung wird mit einer Analysenwaage zweistündig auf die Gewichtskonstanz bei weniger als 0,2\% Massenverlust in den letzten zwei Stunden der Trocknung kontrolliert. Ist sie erreicht, wird die Probenmasse als Bruttotrockenmasse festgehalten und nach Massenbestimmung der entleerten und gereinigten Probenbleche die (Netto-) Trockenmasse der Probe durch Abzug der Probenblechmasse berechnet. Der Wassergehalt kann nun nach folgender Formel ermittelt werden:

$$
w(\%)=\frac{m_{\text {feucht }}-m_{\text {trocken }}}{m_{\text {feucht }}} \cdot 100
$$

Gleichung 2: Berechnung des Wassergehaltes mit:

w:Wassergehalt (\%)

$m_{\text {feucht }}:$ Probenfeuchtmasse $(\mathrm{g})$

$m_{\text {trocken:Probentrockenmasse }(\mathrm{g})}$

Die Wassergehaltsbestimmung des erntefrischen Materials erfolgt zeitnah der Zerkleinerung, die des Materials zur Korngrößenanalyse wenige Tage vor der Korngrößenbestimmung, da sie ausschließlich der Kontrolle dieser dient. 


\section{Korngrößenanalyse}

Die Korngrößenanalyse wird mit Hilfe des mechanischen Rüttelsiebverfahrens nach CEN/TS 15149-1 durchgeführt (CEN/TS 15149-1, 2005). Dazu werden ca. 8 Liter des annähernd lufttrockenen Probenmaterials auf einer Analysenwaage verwogen und mittels einer mechanischen Rüttelsiebanlage fraktioniert. Die schematische Darstellung des Siebvorganges ist in Abb. 17 aufgezeigt. Verwendet werden fünf Lochsiebweiten mit 63, mit 45, mit 16, mit 8 und mit 3,15 mm. Sie werden mit der größten Lochweite von oben her beginnend aufeinander gestellt und eine ungelochte Sammelschale darunter platziert. Der gesamte Siebturm steht auf einer speziellen Rütteleinrichtung. Die Probe wird in die oberste Siebschale gegeben und anschließend 15 Minuten mit Hilfe der Rütteleinrichtung fraktioniert. Nach dem Siebvorgang können die einzelnen Schalen verwogen und nach Abzug der jeweiligen Schalenmasse die Masse der Siebfraktion bestimmt werden. Überlängen $>100 \mathrm{~mm}$ werden manuell aussortiert und verwogen.

Auf diese Weise kann normiert die Masse von acht Größenklassen im Bereich von

1. $>200 \mathrm{~mm}$

2. $>100 \mathrm{~mm}$

3. $100-63 \mathrm{~mm}$

4. $63-45 \mathrm{~mm}$

5. $45-16 \mathrm{~mm}$

6. $16-8 \mathrm{~mm}$

7. $8-3,15 \mathrm{~mm}$ und

8. $<3,15 \mathrm{~mm}$ fraktioniert werden.

Die Siebung wird mit drei Wiederholungen durchgeführt. Die Massenanteile der entsprechenden Siebfraktionen aus den Wiederholungen werden summiert und der prozentuale Anteil an der Gesamtmasse der drei Wiederholungen ermittelt. Die Massenanteile können dann mit den Vorgaben 
der europäischen Norm CEN/TS 14961 „Feste Biobrennstoffe Brennstoffspezifikationen und -klassen" abgeglichen werden.

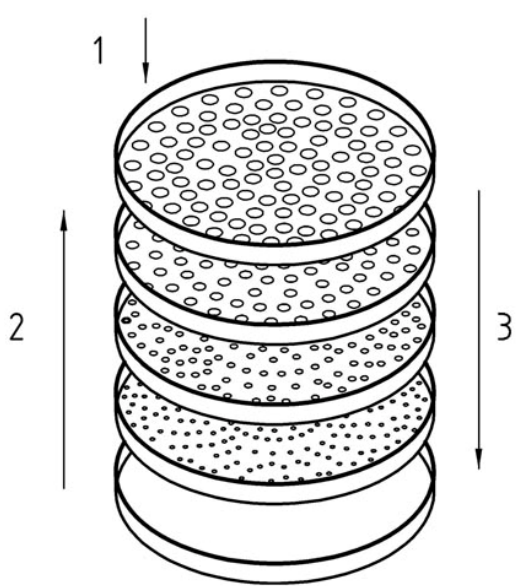

Abb. 17: Schematische Darstellung des Siebvorganges (CEN/TS 15149-1, 2005); 1: Materialzuführung; 2: Zunehmende Lochdurchmesser; 3: Richtung des Materialflusses 


\section{Ergebnisse und Diskussion}

\subsection{Eingrenzung des Verfahrens-/ Zielbereiches}

Ein Ziel der Vorbereitungsphase ist es, den Anwendungsbereich des zu entwickelnden Verfahrens klar einzugrenzen. Dieses stellt nach PonN (2008) eine Grundvoraussetzung dar, um den Rahmen zur Ermittlung der (Produkt-) Anforderungen zu legen, die von einem Konzept später zu gewährleisten sind.

In diesem Fall soll es sich um den Bereich handeln, welcher ein Defizit an Technikverfügbarkeit zur Offenflächenentbuschung aufweist. Da er den Anwendungsbereich des Entwicklungszieles darstellt, soll er folgend als Zielbereich oder Verfahrensbereich mit Verweis auf das zu entwickelnde Konzept bezeichnet werden.

Um Vergleiche zwischen Verfahren durchführen und auch um den Zielbereich definieren zu können, muss eine Basis verwendet werden, die den Anwendungsbereich der Entbuschung von Offenflächen darstellt. Diese muss sich sowohl auf die Entbuschungsverfahren anwenden lassen als auch einen Bezug zur Verbuschung selbst darstellen.

Zu diesem Zweck wird der bearbeitbare Gehölz-Wurzelhalsdurchmesser gewählt. Im Gegensatz zum meist üblichen Brusthöhendurchmesser (1,3 m über Bodenoberfläche) befindet er sich im angestrebten Schnittbereich bei einer Entbuschung und stellt somit eine relevantere Größe hinsichtlich der Bearbeitbarkeit dar. Eine resultierende Entbuschungsleistung einer Technik oder eines Verfahrens wird neben dem WHD der Gehölze auch von weiteren Faktoren, wie der Bestandesdichte, der Gehölzart und letztlich auch den vorzufindenden Flächenbedingungen beeinflusst werden. Derartig aufgeschlüsselte Angaben liegen jedoch in der Literatur für die betrachteten Verfahren nicht oder nur unzureichend vor, so dass ein Einbezug dieser Größen nicht möglich ist.

Anhand Kap. 3.2 sind in Abb. 18 die bearbeitbaren WHD- Bereiche der in der Offenflächenentbuschung eingesetzten Verfahren zusammengefasst. 
Als betrachteter WHD- Bereich der Entbuschung wird der Bereich von $<1 \mathrm{~cm}$ bis $30 \mathrm{~cm}$ gewählt. Die Größe des Bereiches ist dabei weniger dem möglichen Durchmesser von Einzelstämmen sondern eher den mehrtriebigen Wurzelstöcken geschuldet. Diese können bei hoher Triebzahl und entsprechendem Alter trotz relativ geringer Wuchshöhe beachtliche Durchmesser an der Bodenoberfläche erreichen (BÖHMER, 1994).

Es zeichnen sich beim Vergleich Überschneidungen und Überlagerungen zwischen den Verfahren ab, wodurch der gesamte Bereich bis $30 \mathrm{~cm}$ WHD durch die dargestellten Verfahren mehrfach abgedeckt wird.

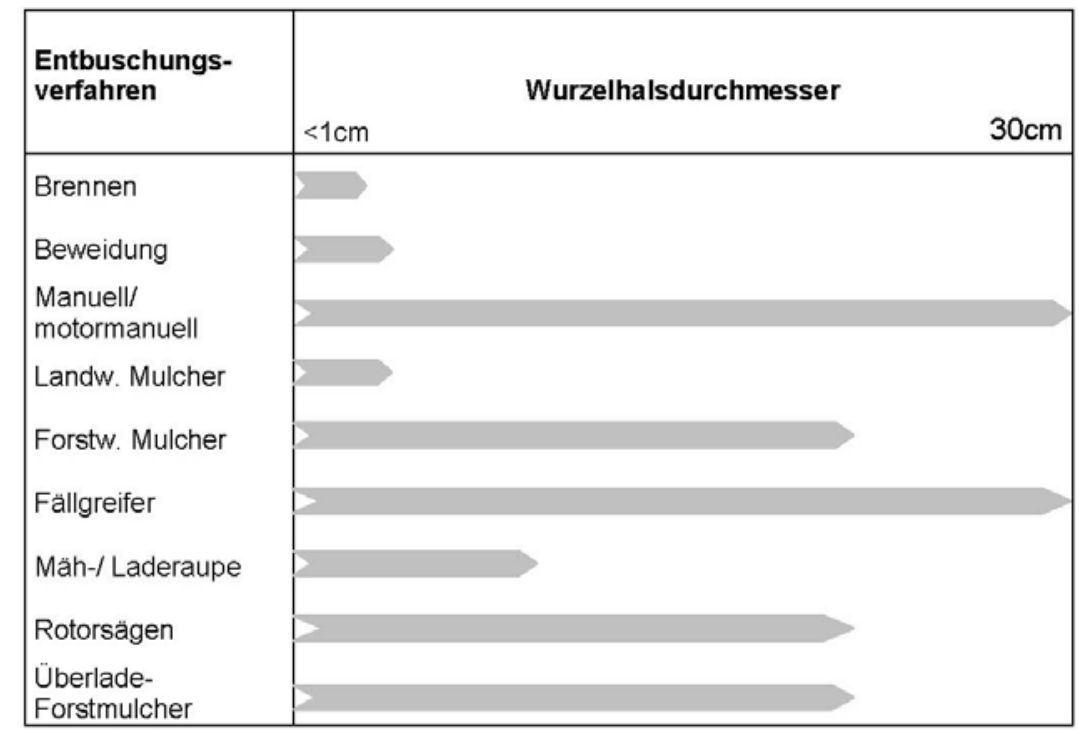

Abb. 18: Technisch bearbeitbare Wurzelhalsdurchmesser von verschiedenen Anwendungsverfahren zur Entbuschung von Offenflächen (Betrachtungsbereich bis $30 \mathrm{~cm}$ )

Würde man diese Betrachtung für sich alleine stehen lassen, würde sich augenscheinlich kein Bedarf an einem weiteren technischen Verfahren zur Offenflächenentbuschung ergeben.

In Tab. 2 sind die verfügbaren Entbuschungsverfahren hinsichtlich ihrer potenziellen Erfüllbarkeit relevanter Ziele bei der Entbuschung zusammengestellt. Einschränkend muss hierbei jedoch auf die mögliche Erfüllbarkeit Wert gelegt werden, da es je nach Fall und Art der Verfahrensanwendung auch zu Abweichungen kommen kann. Beispielsweise kann ein zu hoher Viehbesatz auch durchaus zu einer 
relevanten Gefährdung der Krautschicht führen (RAHMANN, 2000). Es müssen deshalb sowohl günstige als auch praxisgerechte Einsatzbedingungen bei dieser Bewertung vorausgesetzt werden.

Tab. 2: Zusammenfassende Darstellung der potenziellen Erfüllbarkeit ausgewählter Ziele bestehender Entbuschungsverfahren der Offenflächenpflege sowie deren einschränkende Verfahrenseigenschaften + : gegeben; - : nicht gegeben; + - : je Verfahrensausführung bzw. Verbuschungsstruktur;

\begin{tabular}{|c|c|c|c|c|c|}
\hline Verfahren & $\begin{array}{c}\text { geringe } \\
\text { Krautschicht- } \\
\text { gefährdung }\end{array}$ & $\begin{array}{l}\text { Material- } \\
\text { bergung } \\
\text { möglich }\end{array}$ & $\begin{array}{c}\text { allg. } \\
\text { Technikver- } \\
\text { fügbarkeit }\end{array}$ & $\begin{array}{c}\text { allg. } \\
\text { Verfügbarkeit } \\
\text { Fachpersonal }\end{array}$ & Ergonomie \\
\hline Brennen & +- & - & + & - & + \\
\hline Beweidung & + & - & + & + & + \\
\hline $\begin{array}{l}\text { manuell/ } \\
\text { motormanuell }\end{array}$ & + & + & + & +- & - \\
\hline $\begin{array}{l}\text { landw. } \\
\text { Mulcher }\end{array}$ & + & - & + & + & + \\
\hline $\begin{array}{l}\text { forstw. } \\
\text { Mulcher }\end{array}$ & - & - & + & + & + \\
\hline Fällgreifer & + & + & + & + & + \\
\hline $\begin{array}{l}\text { Mäh-I } \\
\text { Laderaupe }\end{array}$ & - & + & - & - & + \\
\hline Rotorsägen & - & + & - & - & +- \\
\hline $\begin{array}{l}\text { Überlade- } \\
\text { Forstmulcher }\end{array}$ & - & + & - & - & + \\
\hline
\end{tabular}

Würden alle dargestellten Zielerfüllungen und Restriktionen bei einem Pflegeeinsatz bindend bzw. gegeben sein, dann würde nach Tab. 2 ausschließlich das Fällgreiferverfahren in der Offenflächenpflege vorzüglich sein. Alle Weiteren weisen ein oder mehrere Defizite oder Restriktionen ihrer Einsatzfähigkeit auf.

Die Verfahren Mäh-/ Laderaupe, Rotorsägen sowie Überlademulcher haben ihr Defizit vornehmlich in ihrer Spezialisierung, weshalb sie allgemein nicht flächendeckend verfügbar sind. Dieses spiegelt sich letztlich auch in ihrer Fachpersonalverfügbarkeit wider. 
Das manuelle/ motormanuelle Verfahren besitzt zwar ein Entbuschungsvermögen über einen weiten Durchmesserbereich, ist jedoch grundsätzlich mit einer schlechten Ergonomie für die Durchführenden und einer eingeschränkten Fachpersonalverfügbarkeit belastet. Die Flächenleistung ist im Verhältnis zu den möglichen Aufwendungen gering. Dieses kann laut PROCHNow \& SCHLAUdERER (2002) sowie LEVIN (2004) auch anhand einer hohen Lohnkostenbelastung deutlich werden. Aufgrund der hohen Technikverfügbarkeit und einer sensitiven und schonenden Einsatzmöglichkeit wird das Verfahren zwar häufig angewandt, es kann aber keine Alternative für den großflächigen Einsatz sein.

Die Verfahren Brennen, Beweidung und landwirtschaftlicher Mulcher erreichen nur ein Entbuschungsvermögen weniger Zentimeter WHD (i.d.R. $<3 \mathrm{~cm}$ ) und lassen verfahrensbedingt keine Materialbergung bei der Entbuschung zu. Das pauschale Ziel einer Materialbergung muss hinsichtlich der bei diesen Durchmesserbereich vorliegenden Gehölze jedoch auch hinterfragt werden. Einen aushagernden Effekt besitzen verfahrensbedingt sowohl das Brennen als auch die Beweidung und aufgrund nur sehr geringer Gehölzmassen wäre vermutlich eine einmalige Auflage ebenso geringer Mengen zerkleinerten Reisigs durch das Mulchen tolerierbar. Hinsichtlich der Bergung und Verwertung würde die Aufarbeitung von derart dünnen Gehölzen sicherlich auch keine Aussicht auf ein verwertbares Endprodukt bestehen lassen, weshalb eine Bergung aus diesem Gesichtspunkt fraglich wäre. Entsprechend dieser Zusammenhänge können diese Verfahren diesen WHD- Bereich der Entbuschung auch sehr gut abdecken. Sie bleiben dabei jedoch insgesamt auf den verhältnismäßig kleinen Durchmesserbereich beschränkt.

Fällgreifer-Verfahren hingegen ließen zwar eine technisches Entbuschungsvermögen bis $<1 \mathrm{~cm} z u$, dieses ist jedoch arbeitswirtschaftlich durch die Einzelbaumbearbeitung nicht zu rechtfertigen. Ihr Arbeitsbereich sollte sich mindestens in einem WHD- Bereich von $5 \mathrm{~cm}$ und mehr bewegen. Werden diese Zusammenhänge in Abb. 18 übertragen, dann zeichnet sich für den Fall, dass alle Ziele und Restriktionen relevant wären, ein anderes 
Bild zur Verfügbarkeit von Entbuschungsverfahren über den betrachteten Anwendungsbereich ab (Abb. 19).

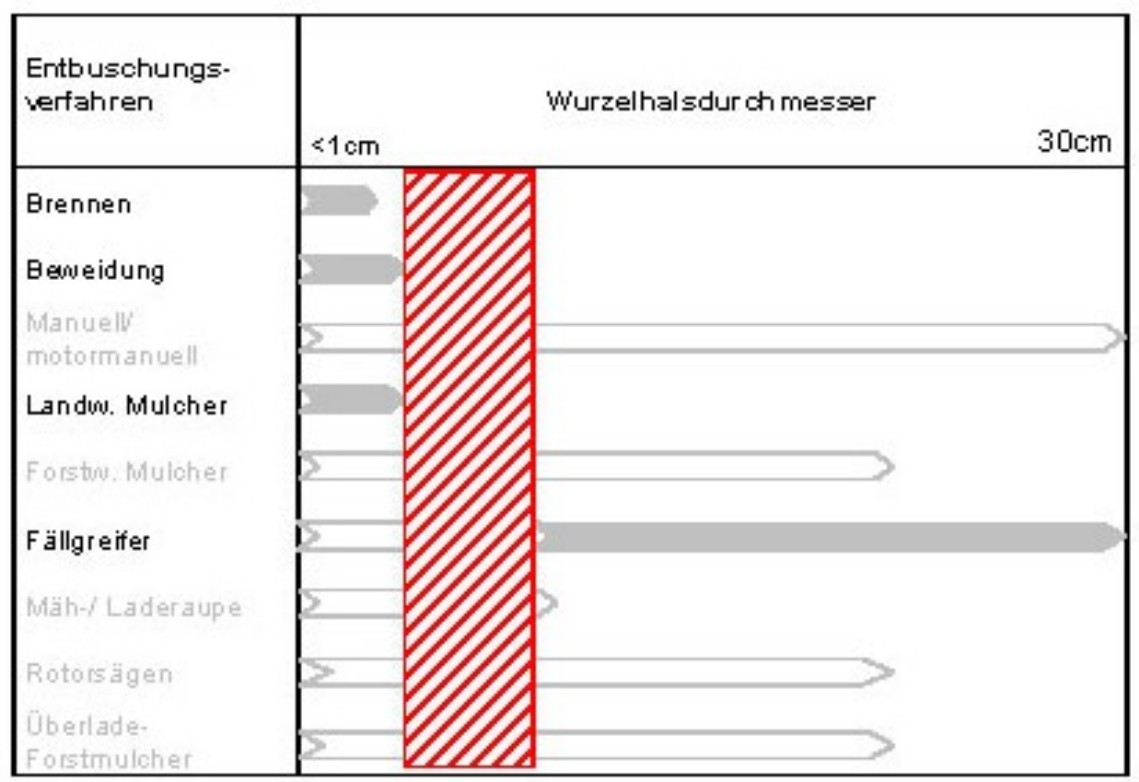

Abb. 19: Bearbeitbare Wurzelhalsdurchmesser von verschiedenen Anwendungsverfahren zur Entbuschung von Offenflächen (Betrachtungsbereich bis $30 \mathrm{~cm}$ ); grau/weiß :

Verfahrensbereich mit Anwendungseinschränkung; rot schraffiert: Bereich eingeschränkter Technikverfügbarkeit(ca. 3- $5 \mathrm{~cm}$ )

Durch Einbezug der Verfahrenseinschränkungen wird im WHD- Bereich von 3- $5 \mathrm{~cm}$ (rot schraffiert) ein Defizit an verfügbarer Verfahrenstechnik deutlich. Auf diesen Durchmesserbereich der Gehölze sollte der Fokus der Entwicklung gesetzt werden. Aufgrund der räumlich und zeitlich „fließenden“ WHD- Entwicklung innerhalb der Sukzession einer Fläche, muss aber eine ausreichende Überschneidung der bearbeitbaren WHD mit den weiteren Verfahren ermöglicht werden. Der Fällgreifer ist verfahrensbedingt erst ab einem WHD von 5 bzw. $10 \mathrm{~cm}$ und darüber hinaus arbeitswirtschaftlich sinnvoll einsetzbar. Da dieses die untere Bearbeitungsgrenze darstellt, sollte der Anwendungsbereich des zu entwickelnden Maschinenkonzeptes sich so weit wie technisch möglich mit diesem überschneiden. Die Notwendigkeit des Einsatzes in diesem Grenzbereich könnte somit weitestgehend vermieden werden. Sinnvollerweise sollte das zu entwickelnde Häckslerkonzept auch 
den gesamten Durchmesserbereich $<3 \mathrm{~cm}$ abdecken, da diese WHD sukzessionsbedingt auch im Bereich von 3- $5 \mathrm{~cm}$ vorkommen würden.

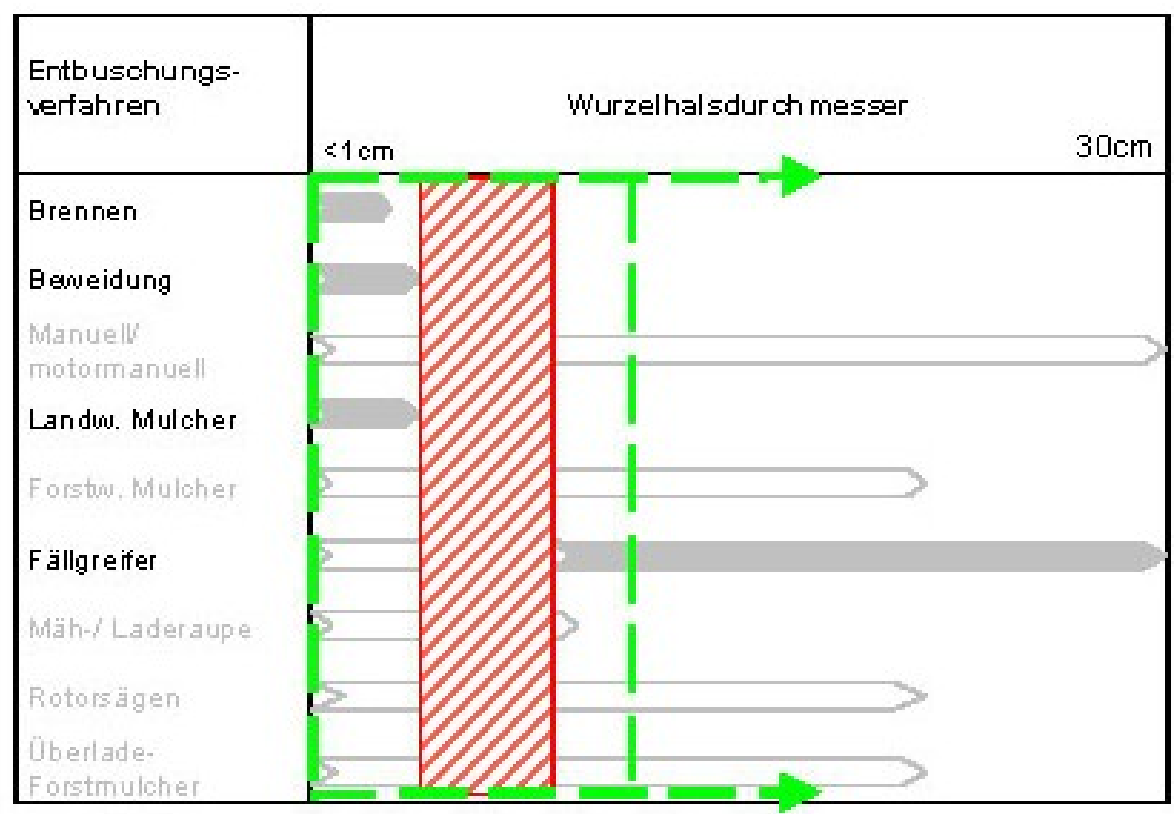

Abb. 20: Bearbeitbare Wurzelhalsdurchmesser von verschiedenen Anwendungsverfahren zur Entbuschung von Offenflächen; Betrachtungsbereich bis $30 \mathrm{~cm}$; grau/weiß: Verfahrensbereich mit Anwendungseinschränkung; rot schraffiert: Bereich eingeschränkter Technikverfügbarkeit (ca. 3- $5 \mathrm{~cm}$ ); grüner Bereich: Anwendungsbereich des zu entwickelnden Maschinenkonzeptes (ca. $<1-10 \mathrm{~cm}$ und darüber)

Das Entbuschungsvermögen des zu entwickelnden Häckslerkonzeptes sollte somit den Bereich von $<1 \mathrm{~cm}$ bis $5 \mathrm{~cm}$ sicher bearbeiten können und eine größtmögliche Bearbeitung von Gehölzen mit einem WHD darüber hinaus ermöglichen. Angestrebt wird hier der Maximalbereich der Bearbeitung von $10 \mathrm{~cm}$ WHD und wenn möglich darüber.

Aufgrund der beschriebenen Sukzessionsdynamik in Kap. 3.1.2 muss auf Sukzessionsflächen beim Erreichen von WHD- Dimensionen um $10 \mathrm{~cm}$ mit dem verbreiteten Vorliegen von WHD im Bereich von $5 \mathrm{~cm}$ und hohen Triebzahlen pro Flächeneinheit zu rechnen sein. Dabei wird sicherlich eine breite Streuung bestehen. Literaturdaten zur Bestätigung dieser Annahme sind aus der Sukzessionsforschung leider nicht genauer zu ermitteln und der Projektrahmen ermöglichte aus Kapazitätsgründen auch keine tiefgreifendere Untersuchung dieses Zusammenhangs. 


\subsection{Anforderungsliste an ein vollmechanisiertes Verfahren zur flächigen Entbuschung von Offenflächen}

Im Entwicklungsprozess wird in diesem Kapitel anhand des ermittelten Zielbereiches der Konzeptanwendung und unter Einbezug von Literaturdaten eine Anforderungsliste erstellt. Um Lösungskonzepte nicht von vorneherein zu stark einzuschränken, sind die hierzu erstellten Anforderungen und ihre Ausprägung bewusst allgemeiner gehalten und auf die Kernelemente reduziert. Im Rahmen der Konzept- und Prototypenentwicklung ist es deshalb auch nicht sinnvoll eine sofortige vollständige Erfüllung anzustreben. Die Anforderungsliste gibt in der Konzeptentwicklung vorerst die Zielrichtung vor, welche zum Abschluss eines gesamten Produktentwicklungsprozesses letztlich erreicht sein muss (LINDEMANN, 2005). Ihre Erfüllung muss bei der Erstellung des Zielkonzeptes im größtmöglichen Maß angestrebt werden.

\subsubsection{Naturschutzfachliche Anforderungen}

Prinzipiell werden die Ansprüche des Naturschutzes und der Landschaftspflege an eine Entbuschungstechnik von dem Pflegeziel, der Schutzwürdigkeit der vorhandenen Biotopelemente sowie auch der vorherrschenden Leitbilder zur Biotopentwicklung/-pflege bestimmt. Dabei ist es notwendig, dass grundsätzliche Anforderungen hinsichtlich des Technikeinsatzes bei Entbuschung von Offenflächen von dem zu entwickelnden vollmechanisierten Maschinenkonzept geleistet werden.

In erster Linie steht hier natürlich das Einsatzziel selbst, nämlich die Freistellung der Fläche, sprich: die Entbuschung. Dabei sollten die Gehölze abgetrennt und auch von der Fläche entfernt werden. Wenn möglich sollte dieses in einem Arbeitsschritt erfolgen, um zusätzliche Arbeitsschritte mit einhergehendem Gefährdungspotential durch weitere Maschinentechnik zu vermeiden. Das Gefährdungspotenzial einer Entbuschungstechnik für die zu erhaltene Flora und Fauna sollte prinzipiell so gering wie möglich sein. Die potenzielle Gefährdung steht dem Mehrwert der Maßnahme gegenüber und entscheidet entsprechend über ihre Einsetzbarkeit. 
Das Abtrennen der Gehölze sollte soweit wie möglich bodennah erfolgen, um einerseits den Wiederaustrieb stärker zu hemmen und andererseits anderen Pflegetechniken (vor allem den Grünlandpflegetechniken) den späteren Einsatz zu ermöglichen. Ein glatter und stockschonender Schnitt, wie er z.B. in der Kurzumtriebsbewirtschaftung für den Wiederaustrieb erforderlich ist (HARTMANN \& THUNEKE, 1997), ist nicht notwendig (DILlENBURGER, 2005).

Grundsätzlich sollte beim Entbuschen ein Bodeneingriff unterbleiben, da dieser einen massiven Eingriff in die Krautschicht darstellt, in der letztlich die zu erhaltene Flora und Fauna bei Offenlandbiotopen fast ausschließlich angesiedelt ist. Eine Freilegung des Oberbodens durch Entfernung oder Zerstörung der Krautschicht kann zwar für die biologische Entwicklung wertvoll sein, sollte jedoch, wenn überhaupt, nur eng begrenzt erfolgen. Auf freigelegten Bodenstellen durch mechanische Schädigungen der Krautschicht können sich Initialstadien der Sukzession auf einem Standort einstellen, welche zu einer gewünschten Diversifizierung beitragen können. Derartige Schäden beinhalten keine Schädigungen, wie sie z.B. durch schädliche mechanische Bodenverdichtungen entstehen. Diese gilt es grundsätzlich zu vermeiden. Vor allem auch, da kurative Maßnahmen, wie sie im Notfall in der Landwirtschaft durch eine entsprechende Bodenbearbeitung möglich wären, auf Landschaftspflegeflächen nicht durchführbar wären, ohne das Biotop zu zerstören (MEINECKE, 2005).

Das Schneiden von Feldgehölzen, darunter fallen die meisten Entbuschungsmaßnahmen auf Offenflächen, unterliegen grundsätzlich den Bestimmungen des Bundesnaturschutzgesetztes (BNatSchG). Sie sind laut $\S 39$ BNatSchG nur in der Zeit vom 01.10. bis zum 28.02. zulässig und fallen somit in die herbst- bzw. winterliche Vegetationsruhe. Nach Aussage der zuständigen Naturschutzbehörde können vereinzelt auch Arbeiten in der Vegetationszeit notwendig werden, wenn dieses die Pflege- und Entwicklungsziele erfordern (siehe auch TAMM, 2008). Es ist somit vornehmlich von Wetter- und Vegetationsbedingungen des Herbst und Winters bei der Entbuschung auszugehen und weniger von denen des Sommers. Bekanntlich sind vor allem die Übergangszeiten von Herbst und 
Winterende von frostfreien und niederschlagsreichen Perioden geprägt. In diesen Zeiten ist mit ungünstigen Flächenverhältnissen für einen Maschineneinsatz zu rechnen, da die allgemeine Tragfähigkeit der Böden dann außerhalb von Trocken- oder Frostperioden stark reduziert sein wird. Die Gefahr von Schlupfschäden und Bodenverdichtungen steigt somit an.

Bei der Aufnahme des Gehölzmaterials muss auf saugende Verfahren verzichtet werden, da diese eine erhebliche Gefahr für Kleinstlebewesen wie Laufkäfer oder Spinnentiere darstellen (LÖBBERT, 2001; WASNER, 1987).

Insgesamt soll sich allgemein nach den folgenden Punkten orientiert werden:

- möglichst geringer Einfluss auf das Biotop mit der zu schützenden Flora und Fauna,

- möglichst geringer Einfluss auf das Bodengefüge,

- bodennahes Absägen der holzigen Vegetation ohne Eingriff in den Oberboden,

Abtransport des Materials in einem Arbeitsschritt,

Verminderung des Nährstoffangebotes des Bodens,

Einsatzfähigkeit in allen Vegetationszuständen eines Jahres.

\subsubsection{Technische und technologische Anforderungen}

Grundsätzlich sollen mit dem Häckslerkonzept flächige Strukturen bearbeitet werden können. Dieses bedingt bei einer Vollmechanisierung die Befahrung der zu bearbeitenden Flächen. Das Basisfahrzeug muss dazu eine größtmögliche Einsatzfähigkeit hinsichtlich begrenzender Einsatzfaktoren wie z.B. Hangneigung, Bodentragfähigkeit aufweisen.

Technologisch muss sich das zu entwickelnde Häckslerkonzept größtmöglich in verfügbare Technikstrukturen einbinden lassen und nicht insgesamt eine Spezialtechnik darstellen, die mit hohen Anschaffungs- oder Bereitstellungskosten verbunden ist. Die Basisfahrzeuge müssen in einem Mindestmaß auf Einsatzbedingungen, wie Baumstümpfen, in den Fahrbereich reichenden Ästen, aufliegendem Astmaterial und 
Dornenmaterial eingerichtet sein. Eine Einbindung des Konzeptes in allgemein bestehende Logistikstrukturen zur Abfuhr des Gehölzmaterials sollen dabei ebenfalls Beachtung finden.

Um der stark propagierten Nutzung von Landschaftspflegematerial entgegen zu kommen sowie auch der Notwendigkeit einer Entsorgung bzw. ungenutzten Beseitigung zu begegnen, sollen die Gehölze direkt zu einer verwertbaren Form aufgearbeitet werden. Zumindest sollte eine Verwertung begünstigt sein. Dabei lassen einphasige Verfahrenstechniken der Entbuschung einen Effizienzvorteil erwarten.

Der Entbuschungsvorgang und die Bergung des Materials sollten ohne den Einsatz händischer Arbeit und vollmechanisiert erfolgen. Daher müssen das Entbuschen und das Bergen lediglich von einem Bediener der Technik durchgeführt werden. Dieses sollte die oftmals beschriebene körperliche sowie auch die Lohnkostenbelastung von Entbuschungsmaßnahmen mindern (PROCHNOW \& SCHLAUdERER, 2002; JeDICKE, 1996).

Pflegeflächen sind oft stark strukturiert und mit Hindernissen, wie aufliegenden Steinen, Senken, Hügeln oder Gräben, durchsetzt (MANN \& BÖHMER, 2008). Des Weiteren können verbleibende Gehölzstrukturen die Befahrung bei der Flächenbearbeitung zusätzlich behindern. Das zu entwickelnde Konzept soll deshalb ein hohes Maß an Manövrierbarkeit und Beweglichkeit auf der Fläche garantieren. Hängigkeit und feuchte Bearbeitungsstandorte sollten bei der Entwicklung Beachtung finden, da unter anderem Hanglagen und anmoorige Standorte von Verbuschung betroffen sind (PERINGER, 2008; BEINLICH, 2002). Das Häckslerkonzept sollte die Eignung der Basismaschine, am Hang oder auf feuchten Standorten zu arbeiten, möglichst gering einschränken.

Um eine sukzessiv gewachsene Gehölzvegetation bearbeiten zu können, muss das eingesetzte Häckslerkonzept mit seiner Technikausstattung auf die unterschiedlichsten Verbuschungsbedingungen eingestellt sein. Sowohl die Wuchsstruktur der Gehölze, die Bestandesstrukturen als auch deren Alter mit einhergehender Größe sind hier zu beachten. Dabei sind prinzipiell unterschiedliche Entwicklungsstadien der Gehölze und ihrer 
Bestandesstrukturen auf einer Fläche zu erwarten, da die Gehölzsukzession in der Regel kontinuierlich, aber in lokal unterschiedlichen Stadien, über einer Fläche voranschreitet (BÖHMER, 1994; PERINGER, 2008). Entsprechend des ermittelten Einsatzbereiches in Kap. 5.1 soll das Konzept Gehölze in einem Bereich bis mindestens $10 \mathrm{~cm}$ WHD bearbeiten können, wobei die Bearbeitbarkeit größerer Durchmesser sicherlich wünschenswert wäre. In diesem Durchmesserbereich muss prinzipiell auch mit hohen Triebzahlen pro Flächeneinheit gerechnet werden, weshalb das Verfahren auch in dieser Vegetation eine gewisse Effizienz aufweisen muss.

Krautiger oder grasiger Unterbewuchs kann zwischen den Gehölzen vorhanden sein. Vor allem in der Vegetationsperiode ist hier mit größeren Massen zu rechnen, welche zudem einen hohen Feuchtegehalt aufweisen können. Dieses durfte bei einer Aufnahme nicht den Aufarbeitungs- oder Austragungsweg behindern.

Bodennahes Abtrennen ohne Bodeneingriff erfordert eine sichere und zuverlässige Höhenführung der Trennorgane. Der Bodenkontakt musste zur Verschleiß- bzw. Schadensvermeidung weitestgehend verhindert werden.

Die Übersichtlichkeit des Konzeptes soll derart gestaltet sein, dass der Bediener den Arbeitsprozess ausreichend und jederzeit beurteilen kann, um auf Störungen notwendige Einstellarbeiten etc. reagieren zu können. Bei einem vollmechanisiertem Konzept muss der Bediener dabei alle möglichen Einstellung, welche während des Betriebs notwenig sind, vom Platz der Bedienung vornehmen können.

\subsubsection{Tabellarische Zusammenfassung}

Die Anforderungen und ihre Ausprägungen sind nachfolgend noch einmal tabellarisch zusammengefasst worden. Dabei werden sie Konzeptmerkmalen zugeordnet und in naturschutzfachliche (Tab. 3) sowie technisch/ technologische Anforderungen (Tab. 4) eingeordnet. 
Tab. 3: Naturschutzfachliche Anforderungen zur Entwicklung eines vollmechanisiertes Häckslerkonzeptes zur Entbuschung von Offenflächen

\begin{tabular}{|c|c|c|}
\hline Konzeptmerkmale & Anforderung & Ausprägung \\
\hline \multirow[t]{6}{*}{$\begin{array}{l}\text { Vollmechanisierter } \\
\text { Technikeinsatz }\end{array}$} & $\begin{array}{l}\text { Flora- und Fauna- } \\
\text { Schonung }\end{array}$ & $\begin{array}{l}\text { - geringstmögliche direkte } \\
\text { Gefährdung/ Zerstörung }\end{array}$ \\
\hline & & - keine nachhaltige Schädigung \\
\hline & & - Verzicht auf saugende Systeme \\
\hline & & $\begin{array}{l}\text { - kein Bearbeitungseingriff in } \\
\text { Oberboden/ Krautschicht }\end{array}$ \\
\hline & Biotopschutz & - vornehmlich vegetationsfreie Zeit \\
\hline & Vegetation & $\begin{array}{l}\text { - vollmechanisierte Bearbeitung von } \\
\text { baum- und buschartiger } \\
\text { Sukzessionsvegetation }\end{array}$ \\
\hline \multirow{3}{*}{$\begin{array}{l}\text { Vollmechanisierter } \\
\text { Gehölzschnitt }\end{array}$} & Abtrennen der Gehölze & - vollständig vom Wurzelstock \\
\hline & & $\begin{array}{l}\text { - kein stockschonender Schnitt } \\
\text { erforderlich }\end{array}$ \\
\hline & & - bodennah \\
\hline \multirow[t]{2}{*}{ Materialbergung } & $\begin{array}{l}\text { Abfuhr des } \\
\text { Gehölzmaterials }\end{array}$ & - möglichst vollständig \\
\hline & & - möglichst in einem Arbeitsgang \\
\hline \multirow[t]{2}{*}{ Flächeneinsatz } & $\begin{array}{l}\text { schonende Befahrung/ } \\
\text { Bodenschonung }\end{array}$ & $\begin{array}{l}\text { - keine schädlichen } \\
\text { Bodenverdichtungen }\end{array}$ \\
\hline & & $\begin{array}{l}\text { - Vermeidung mechanischer } \\
\text { Schädigungen des Oberbodens }\end{array}$ \\
\hline
\end{tabular}


Tab. 4: Technische und technologische Anforderungen zur Entwicklung eines vollmechanisiertes Häckslerkonzeptes zur Entbuschung von Offenflächen

\begin{tabular}{|c|c|c|}
\hline Konzeptmerkmale & Anforderung & Ausprägung \\
\hline \multirow[t]{2}{*}{ Technikausrichtung } & $\begin{array}{l}\text { Einbindung in } \\
\text { bestehende } \\
\text { Technikstrukturen }\end{array}$ & $\begin{array}{l}\text { - Nutzbarkeit allgemein verfügbarer } \\
\text { Trägerfahrzeuge }\end{array}$ \\
\hline & & $\begin{array}{l}\text { - Nutzbarkeit bestehender } \\
\text { Logistikkonzepte }\end{array}$ \\
\hline \multirow[t]{2}{*}{ Technisierung } & Vollmechanisierung & - nur Bediener \\
\hline & & - keine händischen Arbeitsschritte \\
\hline \multirow[t]{3}{*}{ Materialbergung } & $\begin{array}{l}\text { Abfuhr des } \\
\text { Gehölzmaterials }\end{array}$ & - vollständige Bergungsmöglichkeit \\
\hline & & $\begin{array}{l}\text { - Überladung oder Konzentration des } \\
\text { Materials für den Abtransport }\end{array}$ \\
\hline & Aufarbeitung & - möglichst in verwertbarer Form \\
\hline \multirow[t]{3}{*}{ Flächeneinsatz } & flächige Bearbeitung & - Überfahrbarkeit der Fläche \\
\hline & $\begin{array}{l}\text { Manövrierbarkeit/ } \\
\text { Beweglichkeit }\end{array}$ & $\begin{array}{l}\text { - Möglichkeit von Kurvenfahrt im } \\
\text { Arbeitsprozess }\end{array}$ \\
\hline & & - Rangieren auf engem Raum \\
\hline \multirow[t]{7}{*}{$\begin{array}{l}\text { Vollmechanisierter } \\
\text { Entbuschungsvorgang }\end{array}$} & Schneiden der Gehölze & $\begin{array}{l}\text { - vollständig bis mindestens } 10 \mathrm{~cm} \\
\text { WHD und möglichst darüber }\end{array}$ \\
\hline & & - Schnitthöhe beeinflussbar \\
\hline & Aufnahme der Gehölze & $\begin{array}{l}\text { - Aufnahme und sichere Übergabe an } \\
\text { die Aufbereitung }\end{array}$ \\
\hline & $\begin{array}{l}\text { Aufbereitung der } \\
\text { Gehölze }\end{array}$ & - energieeffizient \\
\hline & & - transportwürdige Aufarbeitung \\
\hline & & - logistisch vorteilhaft \\
\hline & & - möglichst verwertbares Endprodukt \\
\hline \multirow[t]{2}{*}{ Bedienung } & Ferneinstellung & $\begin{array}{l}\text { - alle Betriebsfunktionen vom } \\
\text { Bedienplatz einstellbar }\end{array}$ \\
\hline & Übersichtlichkeit & $\begin{array}{l}\text { - alle Betriebszustände vom } \\
\text { Bedienplatz ausreichend beurteilbar }\end{array}$ \\
\hline Sicherheit & Arbeitssicherheit & $\begin{array}{l}\text { - größtmöglicher Schutz des } \\
\text { Bedienpersonals } \\
\end{array}$ \\
\hline \multirow[t]{2}{*}{ Haltbarkeit } & Verschleiß & - möglichst hohe Standzeit \\
\hline & Festigkeit & $\begin{array}{l}\text { - ausreichende Stabilität der } \\
\text { Komponenten }\end{array}$ \\
\hline
\end{tabular}

\subsection{Basiskonzept}

Die Grundüberlegung des Basiskonzeptes muss von verfahrenstechnischer Seite vom Gegenstand der Betrachtung, also der Verbuschung auf der Offenfläche bzw. deren flächiger Bearbeitung ausgehen. 
Der Anwendungsbereich bei einem WHD von <1- $10 \mathrm{~cm}$ lässt entsprechend des Sukzessionsverlaufs auf das Vorkommen einer relativ hohe Anzahl Triebe pro Flächeneinheit einhergehend mit geringer Masse pro Trieb schließen. Hier sind unselektiv arbeitende Mähverfahren im Gegensatz zum Fällgreiferverfahren deutlich im Vorteil. Es muss dabei aber speziell auf das Schneiden von Gehölzen bei Entbuschungen ausgelegt sein. In einem ähnlichen Arbeitsbereich, bei der Beerntung von Kurzumtriebsplantagen, konnte ein entsprechender Effizienzzusammenhang beim Einsatz von Mähtechnik festgestellt werden (BURGER, 2004; SCHOLZ, 2007). Die dort eingesetzten Maschinentechniken wären in diesen Einsatzbereich jedoch nicht übertragbar, da es sich um reihengebundene Erntetechniken handelt. Bei der Entbuschung von Offenflächen darf die Mähtechnik keine Reihenbindung besitzen.

Eine Mähtechnik zum Mähen von Gehölzen könnte prinzipiell über verschiedene Schneidtechniken erfolgen. Es wären spanlose sowie spanende Techniken mit linearen oder auch zirkulär arbeitenden Messern möglich (BÄRISCH ET AL., 1982; PERSSON, 1987). BLOCK (2004) kann über den Mäheinsatz in tropischer Gehölzvegetation von sehr guten Erfahrungen beim Schneiden mit Sägeblättern berichten. Diese bewähren sich dort auch unter extremen Bedingungen zuverlässig. Auch bei den Mähverfahren der Kurzumtriebsplantagen werden fast ausschließlich nur Sägeblätter eingesetzt. Aufgrund der durchaus vergleichbaren Anforderungen beim Schneiden von Gehölzen bei der Entbuschung in einem Mähvorgang sowie auch hier auftretendem grasigen und krautigem Material sollte der Schnitt somit durch zirkulär arbeitende Kreissägeblätter erfolgen. Kreissägeblätter kennzeichnet ein verhältnismäßig glattes Schnittbild aus und sie besitzen eine hohe Standfestigkeit. Beim Schnitt können sie auch vertikalen Kräften widerstehen (FELDE ET AL., 1980), wie sie bei einer waagerecht angeordneten Verwendung in einem mähenden Einsatz durch das Gewicht aufstehender Gehölze zu erwarten sind. Durch den glatten Schnitt wird keine Hemmung des Wiederaustriebs zu erwarten sein, jedoch würden fräsende Verfahren (beispielsweise ein gegenläufig zur Arbeitsrichtung drehendes Forstmulcherprinzip) zum Abtrennen der Gehölze das Risiko einer 
Krautschichtgefährdung erhöhen. Deren Einsatzmöglichkeit wird zwar auch hinsichtlich der Widerstandsfähigkeit gegenüber Störstoffen, wie aufliegenden Steinen oder Sand, diskutiert, letztlich aber auch wegen des zu erwartenden hohen Leistungsbedarfes zum Abtrennen der Gehölze verworfen (SCHMIDT, 2008).

Die Art der Führung des Maschinenkonzeptes im Arbeitsprozess wäre prinzipiell handgeführt oder maschinengeführt möglich. Handgeführte Maschinenkonzepte müssen in diesem Fall ausscheiden, da sie letztlich in den motormanuellen Bereich mit den einhergehenden Restriktionen (Ergonomie, Bedienersicherheit) fallen würden. Eine Maschinenführung kann über Kranausleger oder über eine direkte Ankopplung an ein Basisfahrzeug realisiert werden. Bei einer direkten Ankopplung an ein Basisfahrzeug ist es notwendig, die zu bearbeitenden Gehölze in irgendeiner Form anzufahren und das Basisfahrzeug muss bei jeder Arbeitsbewegung mit versetzt werden. Auslegergeführte Maschinenkonzepte können dagegen einen gewissen Arbeitsbereich abdecken, ohne ihn befahren zu müssen. Dabei können sie auch technisch nicht befahrbare Bereiche in Weite der Kranauslage bearbeiten und ermöglichen durch die höhere Beweglichkeit eine feinstrukturierter Bearbeitung (BÄRISCH ET AL., 1982). Mögliche Standardbasisfahrzeuge, wie Bagger oder Forstkräne, erreichen heute zwar Traglasten bis zu einer Tonne und auch darüber hinaus, jedoch lässt sich eine günstige Materialbergung in dem angestrebten Arbeitsbereich an Auslegersystemen nur bedingt realisieren. Eine Materialbergung auf der Offenfläche kann in unbearbeiteter Stammform als auch in aufbereiteter Form wie z.B. Hackschnitzeln erfolgen. Unbearbeitet muss das Konzept zur Bergung mindestens das Sammeln und eine Vorkonzentration des Materials ermöglichen, um einen Effizienzvorteil gegenüber den Rotorsägen zu erlangen (siehe Kap. 3.2.3.2). Die eigentliche Bergung und auch Aufarbeitung des Materials muss dann in weiteren Schritten erfolgen, welche es gemäß der Anforderungsliste zu vermeiden gilt. Eine direkte Aufarbeitung zu einem transportwürdigem und möglichst verwertbarem Gehölzmaterial sowie dessen Bergung direkt nach dem Schneiden muss somit Teil des Konzeptes sein. Aufgrund des hohen zu erwartenden Volumens pro 
Masseneinheit (RAHMANN, 2000) und der eher minderen Holzbeschaffenheit natürlich gewachsener Vegetation ist eine direkte Aufarbeitung zu Holzhackschnitzeln voraussichtlich die günstigste Variante (HAKKILA, 1989); (Abb. 21). Ein nennenswerter Anteil an verwertbarem Stammholz ist aufgrund des Durchmesserbereiches und der vorherrschenden Holzarten prinzipiell nicht zu erwarten und Hackschnitzel erreichen als umschlagfähiges Massengut eine akzeptable Transportwürdigkeit (CREMER ET AL., 2007).

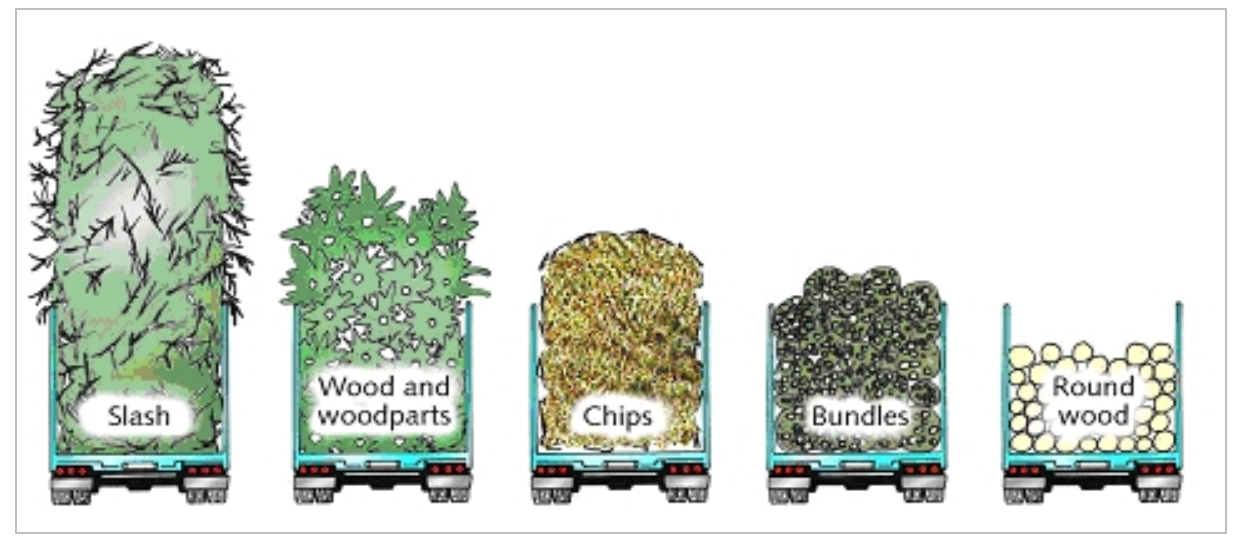

Abb. 21: Transportvolumen der gleichen Menge Holz in Abhängigkeit von der Beschaffenheit (Quelle: Timperi; A.; Finnland)

Die gleichzeitige Aufarbeitung der Gehölze bei der Entbuschung setzt voraus, dass ein Hackaggregat in dem Konzept integriert ist. Bei einem auslegergeführten Maschinenkonzept muss dieses mit am Ausleger befestigt sein oder der Entbuschungsvorgang muss als Verfahren das Mähen und das gleichzeitige Sammeln mit dem Ausleger sowie anschließend ein phasenweises Beschicken eines mitgeführten Holzhackers ermöglichen. Auf diese Arten könnte ein einphasiges Konzept in einem Arbeitsgang verwirklicht werden. Das phasenweise Beschicken eines separaten Holzhackers mit einem Gehölzmähkopf einer für eine Flächenleistung ausreichenden Arbeitsbreite erscheint jedoch nur schwer möglich, da Praktiker schon von Platzproblemen beim Beschicken von Holzhackern mit Fällgreifern berichten (HEIKE, 2008). Bei einem Auslegersystem mit Häcksel- und Mähfunktion ließe sich die Hackschnitzelbergung des Weiteren nur saugend ermöglichen, wie es z.B. bei dem Auslegerhäckslersystem von der Fa. MULAG für die Straßenrandpflege der Fall ist (Abb. 22). Dieses 
würde der naturschutzfachlichen Anforderung auf den Verzicht derartiger Systeme in der Offenflächenentbuschung zuwider laufen. Auch technisch würde ein derartiges Auslegersystem mit Schneid- Häckselfunktion an Grenzen stoßen, da es im angestrebten Gehölzdurchmesserbereich weit massiver ausgeführt sein müsste als z.B. das MULAG- System. Dieses erreicht bei einer Arbeitsbreite von $1,2 \mathrm{~m}$ und $310 \mathrm{~kg}$ Gewicht einen maximalen Gehölzdurchmesser von $2 \mathrm{~cm}$. Bei einer Auslegung des Systems auf einen WHD von $10 \mathrm{~cm}$ oder mehr und einer entsprechenden Arbeitsbreite würde allein schon das zu erwartende Gewicht am Ende des Auslegers problematisch werden. Reine handbeschickbare Anbauhäcksler zur Bearbeitung dieses Durchmesserbereiches erreichen Gewichte über $500 \mathrm{~kg}$. Diesem Gewicht müssen noch Mäh- und Bergetechnik hinzugerechnet werden.

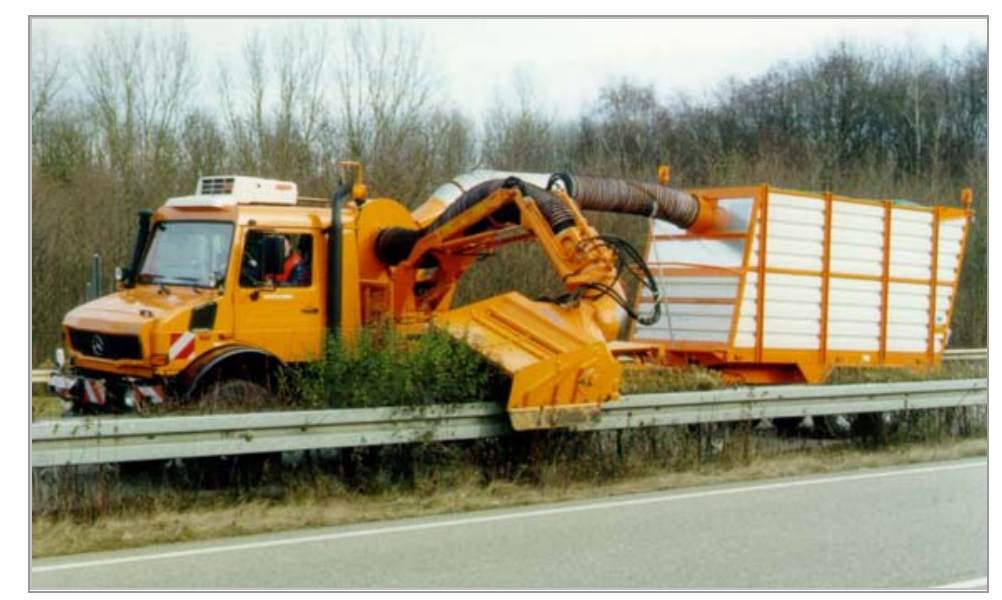

Abb. 22: MULAG Heckenschneidkopf HSK 1200 bei der AutobahnMittelstreifenpflege mit gleichzeitiger Absaugung des Häckselmaterials (MULAG FAHRZEUGWERK HEINZ WÖSSNER, 2006)

Die direkte Ankopplung einer Maschine, in der Mäh- und Zerkleinerungseinheit untergebracht sind, an ein geeignetes Basisfahrzeug erscheint somit als die einzig adäquate Möglichkeit ein flächig arbeitendes Häckslerkonzept zu verwirklichen.

Für den Aufbau des Zerkleinerungsaggregates stehen die gängigen Prinzipien Scheibenrad-, Trommel- und Schneckenhacker zur Verfügung. Grundsätzlich lassen sich mit allen dieser Zerkleinerungsverfahren 
zufriedenstellende Ergebnisse bei der Holzzerkleinerung erzielen. Scheibenrad- und Trommelhacker benötigen Zuführaggregate zum gleichmäßigen Einzug von sperrigem Material im Hackvorgang, da die Einzugskräfte durch den Hackvorgang nicht immer ausreichend sind. Aufgrund dessen soll die Zerkleinerung mit einem Schneckenhackeraggregat erfolgen. Durch den Selbsteinzug kann im Hackvorgang auf die zusätzliche Förderung des Gehölzmaterials zur direkten Beschickung des Häckselvorganges verzichtet werden. Eventuelle Zuführaggregate des Häckslerkonzeptes können sich somit auf die Konzentration des Materials vor der Schneideinheit und den Transport zur Häckseleinheit beschränken. Nach BRUSCHE (1983) und NURMI (1986) weisen Schneckenhacker einen relativ geringen Energieaufwand bei der Zerkleinerung von Holz im Bereich von $10 \mathrm{~cm}$ Durchmesser auf und produzieren Hackschnitzel mit einer vergleichsweise engen Hackschnitzelgrößenverteilung. Durch die Möglichkeit zur Produktion von Grobhackgut (Hackschnitzellängen $>50 \mathrm{~mm}$ ) ist es weiterhin möglich, ein geeignetes Material zur späteren Lagerung und Trocknung herzustellen (SCHOLZ \& IDLER, 2005). Da generell aufgrund der Ausgangsmaterialbeschaffenheit (Feinastanteil, Aschegehalt, Gras-/ Krautaufnahme etc.) von einem qualitativ schlechteren Endprodukt mit geringerer Marktleistung ausgegangen werden muss, als es z.B. bei Waldhackschnitzeln zu erwarten ist, soll eine Qualitätsverbesserung durch natürliche Trocknung oder eine weitere Qualitätsminderung durch eine schlechte Lagerfähigkeit von Feinhackschnitzeln (SCHOLZ ET AL., 2005) vermieden werden. BRUSCHE (1983) rät nach seinen Untersuchungen vom Einsatz des Schneckenhackers bei Ästen und Reisig eher ab, führt diese Aussage jedoch ausschließlich auf den verengten Einzug des bei ihm verwendeten Gerätes zurück. Dieses muss bei einer technischen Umsetzung berücksichtigt werden und sich letztlich in den Praxiseinsätzen ermitteln lassen.

Zur Bergung des Hackmaterials auf der Offenfläche muss dieses vom Hackaggregat in einen geeigneten Transportbehälter verladen werden. Bei mobilen Anwendungen wie Feldhäckslern hat sich zur Gewichtsersparnis 
und auch zur Flexibilität bei der Verladung des Hackgutes das Wurfgebläse mit einem flexibel einstellbaren Auswurfturm etabliert.

Die Beschleunigung des Gutes erfolgt im Gegensatz zu Druckgebläsen vornehmlich durch einen Impuls der Wurfschaufeln und weniger durch einen Luftstrom. Je nach Beschleunigergestaltung kann der Anteil des Luftstroms zur Unterstützung der Förderung verschieden groß sein. Das Überladegut verlässt idealerweise als konzentrierter und gerichteter Gutstrom die Mündung des Auswurfturmes und kann über eine gewisse Distanz transportiert und in einem entsprechenden Behälter o.ä. aufgefangen werden. Andere Stetigförderer, wie Zugbandförderer oder Schneckenförderer besitzen zwar auch hohe Förderleistungen und haben dabei einen relativ geringen Energiebedarf, sie sind dabei aber baulich relativ groß und schwer, wenn sie über gewisse Distanzen frei hängend realisiert werden müssen. Bei nur kleinen Überwurfweiten am Ende der Förderer müssen sie funktionsbedingt annähernd von Anfang bis Ende der Förderstrecke reichen und der Übergabepunkt ist nur begrenzt veränderbar (MARTIN ET AL., 2008). Für einen mobilen Einsatz auf der Fläche können deshalb nur Wurfgebläse zum Überladen des Materials Verwendung finden, obwohl ihr Leistungsbedarf zur Verladung höher ist. Da der Luftstromanteil konstruktiv gering gehalten werden kann, wären Gefährdungen durch Saugeffekte ausschließbar.

Die flächige Bearbeitung setzt voraus, dass die Fläche mit einem geeigneten Träger- oder Zugfahrzeug für die Bearbeitungstechnik befahren werden muss. Bei der Entwicklung einer vollmechanisierten Entbuschungstechnik für sensible Standorte und dem Vorkommen von extremeren Flächenbedingungen, wie starken Hangneigungen oder bedingt tragfähigen Böden, würde der Einsatz eines spezial angepassten Fahrzeuges, wie z.B. der Pistenraupe, sicherlich einen Vorteil darstellen. Hinsichtlich einer späteren Technikverfügbarkeit und auch der Entwicklungs- und letztlich wiederum der darauf übergehenden Anschaffungskosten einer solchen Spezialtechnik würde sich jedoch in der Anwendung ein gravierender Nachteil einstellen. 
Um dieses zu umgehen, bieten sich nur land- oder forstwirtschaftliche Schlepper (synonym: Traktoren) an, deren Verwendung aus den folgenden Gründen weiter verfolgt wird. Schlepper sind flächendeckend und in unterschiedlichsten Ausstattungs- und Leistungsvarianten verfügbar und können unter vielfältigsten Einsatzbedingen eingesetzt werden. Hangneigungen sind mit heutigen Schleppern und entsprechender Anpassung bis zu Steigungen von $44 \%$ bearbeitbar (SAUTER ET AL., 2008) und durch den Einsatz leichter und mit entsprechenden Reifen ausgestatteter Traktoren lassen sich auch sehr feuchte Standorte bedingt befahren. Dieses schränkt das Einsatzgebiet einer schlepperbasierten Häckslertechnik zwar vergleichsweise ein, daneben führen Land- und Forstwirte aber auch heute schon einen nicht unerheblichen Anteil der Entbuschungsmaßnahmen mit inren Traktoren durch. Deshalb sind sie einerseits mit der Pflege und andererseits mit den Fahrzeugen vertraut und stellen somit auch einen großen potenziellen Anwenderkreis des Häckslerkonzeptes dar. Da Entbuschungsmaßnahmen vornehmlich in der vegetationsfreien Zeit durchgeführt werden, wäre außerdem eine zusätzliche Maschinenauslastung möglich, was die Akzeptanz einer schlepperbasierten Technik steigern würde.

Einschränkend muss bei der Ausrichtung auf land- und forstwirtschaftliche Schlepper gesagt werden, dass grundsätzlich ein Mindestmaß an technischer Umrüstung zur technischen aber auch zur Sicherheit des Bedieners unumgänglich sein würde. Da es sich z.B. um verstärkte Reifen und zusätzliche Schutzbügel gegen Äste handeln würde, beträfe dieses vor allem landwirtschaftliche Schlepper. Ein schlepperbasiertes Häckslerkonzept würde aber insgesamt der Anforderung an eine Einbindbarkeit in bestehende Technikstrukturen am Nächsten kommen. Hinsichtlich der Anforderungen an Ergonomie und Bedienung wäre durch umfangreiche hydraulische Steuermöglichkeiten heutiger Schlepper sowie einer übersichtlichkeits- und ergonomiebetonten Arbeitsplatzausstattung auch eine ausreichende Erfüllung dieser Anforderungen möglich. 
Nach der Festlegung auf ein schlepperbasiertes Konzept muss dessen günstigste Anbindung bestimmt werden. Standardtraktoren lassen das Arbeiten mit zwei verschiedenen Arten von Zusatzgeräten zu. Dieses sind Anbau- und Anhängegeräte. Anhängegeräte werden an einem seitlich- und höhenschwenkbaren Koppelpunkt (z.B. Bolzen- oder Kugelanhängung) angehängt und tragen mindestens einen Teil ihres Gewichtes durch ein eigenes Fahrwerk. Anbaugeräte dagegen werden meist in der Drei- PunktHydraulik des Traktors fix angekuppelt und vom Traktor getragen. Dadurch belasten sie zwar zusätzlich die Hinterräder des Schleppers, können aber baulich näher am Schlepper gehalten werden, da auf den Schwenkraum zwischen Traktor und Gerät verzichtet werden kann, der für Kurvenfahrten benötigt wird. Durch die Möglichkeit zur kürzeren Bauweise wäre die Manövrierbarkeit auf engem Raum mit Anbaumaschinen somit eher gegeben und durch die fixe Kupplung auch eine bessere Spurtreue gegen seitlichen Schlupf in hängigem Gelände. Entscheidender für die Bevorzugung einer angebauten Bauweise ist jedoch, dass ein Anhängegerät ähnlich eines Anhängers rückwärts geschoben oder seitlich versetzt zur Spur des Schleppers gezogen werden müsste, ohne bei der Entbuschung über den stehenden Bestand fahren zu müssen. Eine Höhenführung zur Einstellung der Schnitthöhe wäre außerdem über die Drei-Punkt- Hydraulik des Schleppers vom Bedienplatz aus möglich.

Die angebaute Bauweise kann jedoch nur in Schubfahrt erfolgen, da sonst mit dem Schlepper vor der Bearbeitung über die Gehölze gefahren werden müsste. Diese Festlegung hat für den Betrieb aber auch die Konsequenz, dass eine Arbeitsbreite bzw. Mähbreite erreicht werden muss, die mindestens der Schlepperbreite entspricht. Zur verbesserten Manövrierung bei beidseitig stehendem Bestand wäre eine darüber hinausgehende Breite sicherlich vorteilhaft.

Eine einphasige Bearbeitung setzt eine gleichzeitige Bergung des Hackschnitzelmaterials bei der Entbuschung voraus. Nach HARTMANN \& THUNECKE (1997) können bei der einphasigen Gehölzernte in Kurzumtriebsplantagen das Parallelverfahren mit einem direkten Überladen 
auf ein weiteres eigenständiges Transportfahrzeug, das Umhängeverfahren mit dem Auswechseln von angekuppelten Transportanhängern sowie das Bunkerverfahren mit einer Zwischenspeicherung auf dem Erntefahrzeug eingesetzt werden. Bei letzterem wird das Material bei Erreichen der Bunkerkapazität auf ein abseits stehendes Transportfahrzeug verladen und der Bediener agiert auf der Fläche sowie beim Verladen allein. Das Verladen entfällt durch das Auswechseln beim Umhängeverfahren, es muss jedoch bei beiden der Arbeitsprozess unterbrochen werden, während er beim Parallelverfahren kontinuierlich fortgeführt werden kann. Aufgrund dieser Zeitersparnis erreicht das Parallelverfahren allgemein eine höhere Flächenleistung. Bei der Entbuschung herrschen im Vergleich zur Beerntung von Kurzumtriebsplantagen jedoch andere Bearbeitungsverhältnisse. Es ist z.B. mit einem weit geringerem Materialaufkommen pro Flächeneinheit zu rechnen. Der Vergleich der ermittelten Gehölzmassen von RaHMANN (2000) bzW. von MANN \& BÖHMER (2008) mit üblichen Erntemengen der Kurzumtriebsplantagenbewirtschaftung im Bereich von $100 \mathrm{t}_{\mathrm{FM}} /$ ha ergibt, dass die zu erwartenden Gehölzmassen bei der Entbuschung ungefähr nur ein Fünftel bis ein Zehntel betragen. Bei dem geringen Materialaufkommen relativiert sich der Zeitvorteil des direkten Verladens und der negative Einfluss der verminderten Manövrierbarkeit und der notwendigen Abstimmung zwischen den Akteuren wird zudem erhöht. Die natürlich gewachsenen Gehölzstrukturen besitzen keine klar festgelegten Bearbeitungsmuster und die teils beengten Verhältnisse erhöhen den Anspruch zum Rangieren etc.. Ein Bunkerverfahren mit einem vom Basisfahrzeug getragenen Transport- bzw. Überladebehälter erscheint somit die beste Lösung. Auf diese Weise kann eine größtmögliche Manövrierfähigkeit erhalten bleiben und Abstimmungen zwischen mehreren Akteuren sind überflüssig, da der Bergungsprozess auf den Bediener der Entbuschungstechnik begrenzt bleibt. Aus Gewichtsgründen und zur Beschränkung der Systemabmaße sollte der Bunker nur dem Zwischentransport auf der Fläche dienen und zum Abtransport vom Einsatzort eine schnelle Entladung auf übliche Transportfahrzeuge ermöglichen. Eine Entladung ist somit auch auf befestigten Wegen oder auf 
Flächen abseits der Pflegefläche möglich, ohne diese zusätzlich befahren zu müssen. Die Bunkerkapazität muss zwischen der zusätzlichen Bodenbelastung, einer Einschränkung der Fahreigenschaften sowie einem möglichst weitem Entladeintervall bestimmt werden.

Das Basiskonzept zur vollmechanisierten Entbuschung von Offenflächen lässt sich entsprechend dieser Überlegungen folgendermaßen zusammenfassen:

- $\quad$ ein vom Basisfahrzeug geschobenes Mähverfahren für Gehölze

- Schneiden der Gehölze mittels Sägeblättern über die Breite des Basisfahrzeuges oder darüber hinaus

- einphasige Aufarbeitung der Gehölze zu Holzhackschnitzeln direkt nach dem Schneiden

Zerkleinerung mittels Schneckenhackertechnik

land- oder forstwirtschaftliche Standardschlepper in leicht modifizierter Form als Basisfahrzeuge

Anhängesystem in der Drei- Punkt- Hydraulik eines land- oder forstwirtschaftlichen Schleppers

- Bergung und Verladung auf der Fläche in einen Zwischenbunker mittels Wurfgebläse

schnelle Entladung auf oder neben der Fläche auf ein geeignetes Transportfahrzeug zum Abtransport vom Einsatzort

\subsubsection{Potenzielle Eignung des selbstschneidenden Häckslerkonzeptes „Tritucap 2" zur Entbuschung von Offenflächen}

Das Maschinenkonzept der Tritucap 2 erscheint bei erster Betrachtung einen weiten Bereich der Kernpunkte des Basiskonzeptes zu erfüllen. Der Betrieb ist schlepperbasiert und die Maschine ist für eine Bearbeitung natürlich gewachsener holziger sowie krautiger Biomasse konzipiert. Dabei arbeitet die Maschine in Schubfahrt und auf Schlepperbreite von 2,1 m. Die 
Zerkleinerung erfolgt nach dem Schneckenhackerprinzip, welches bei diesem Maschinenkonzept durch die auf den Sägeblättern stehenden senkrechten Häckselrotoren jedoch eine spezielle Form eines Schneckenhackers darstellt. In seinem Aufbau sieht das Maschinenkonzept aber keine Bergung der bearbeiteten Biomasse vor, sondern einen Aufschluss als Mulchmaterial mit anschließender Breitverteilung. Entsprechend ist technisch auch keine Bergungs- bzw. Überladetechnik vorgesehen.

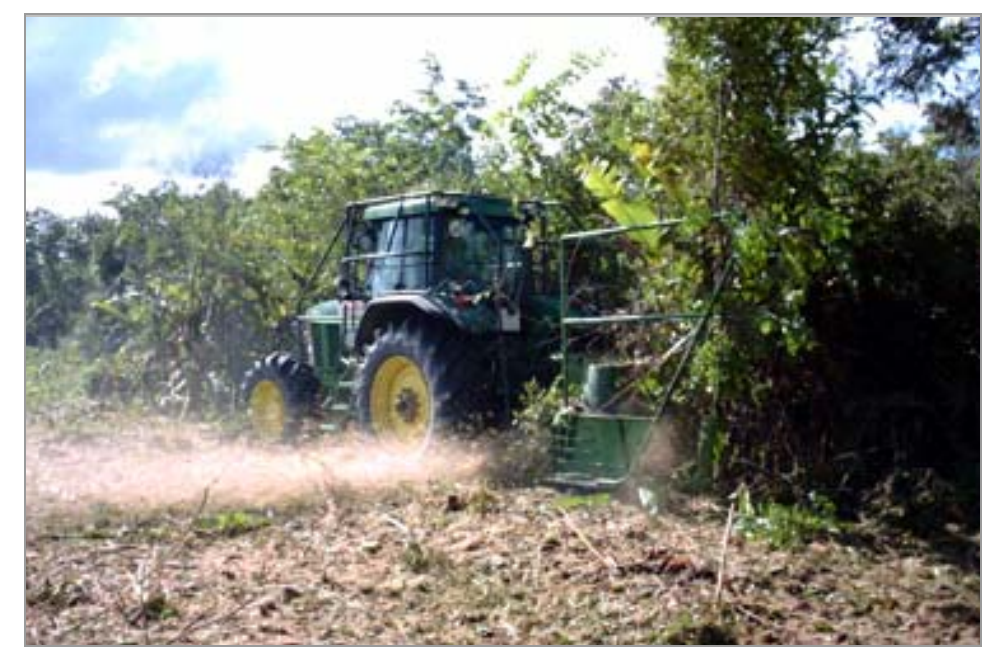

Abb. 23: Selbstschneidendes Häckslersystem „Tritucap 2“ bei der Flächenbereitung im Rahmen der nichtbrennenden Mulchwirtschaft in Amazonien (Quelle: Block)

Die Einsatzbedingungen in brasilianischer Sekundärwaldvegetation lassen sich sicherlich nicht direkt auf Verbuschungen mitteleuropäischer Sukzessionsflächen übertragen. Allerdings lagen im dortigen Einsatz Gehölzdurchmesser im Bereich von $<1 \mathrm{~cm}$ bis $10 \mathrm{~cm}$ sowie baum- als auch buschartige Wuchsformen der Gehölze in unterschiedlichsten Entwicklungsstadien vor (BLOCK, 2004), welche dem angestrebten Zielbereich dieser Arbeit nahe kommen. Die Tritucap ermöglicht dort in umfangreichen Untersuchungen das flächige Schneiden und Zerkleinern derartiger Vegetation, weshalb ein aussichtsreicher Einsatz in dem hier behandelten Bereich angenommen werden kann.

BLOCK (2004) analysiert in seinen Arbeiten auch die Qualität des aufgearbeiteten Materials. Dabei wird ein hoher Massenanteil Hackschnitzel 
von ca. $75 \%$ im Größenbereich unter $35 \mathrm{~mm}$ sowie auch große Anteile an „zerfasert" und „strukturlos" aufgearbeitetem Material festgestellt (siehe Abb. 24). Eine derartige Aufarbeitungsqualität entspräche nicht einer Eignung zur Herstellung eines nach Hackschnitzelnorm klassierbaren Verwertungsmaterials. Vorzügliche Hackschnitzel weisen glatte Schnittflächen und eine möglichst kantige Form auf (SCHOLZ ET AL., 2006) anstatt zerfaserter Strukturen. Die Größenverteilung müsste des Weiteren eingeschränkter sein, um z.B. der Hackschnitzelnorm CEN/TS 14961, 2005 zu genügen. BLOCK (2004) gibt in seinen Arbeiten an, dass auch wesentliche Anteile an weniger verholzter Biomasse aufgearbeitet wurden. Inwieweit dieses einen Einfluss auf die Aufarbeitungsergebnisse hat, lässt sich jedoch nicht entnehmen.

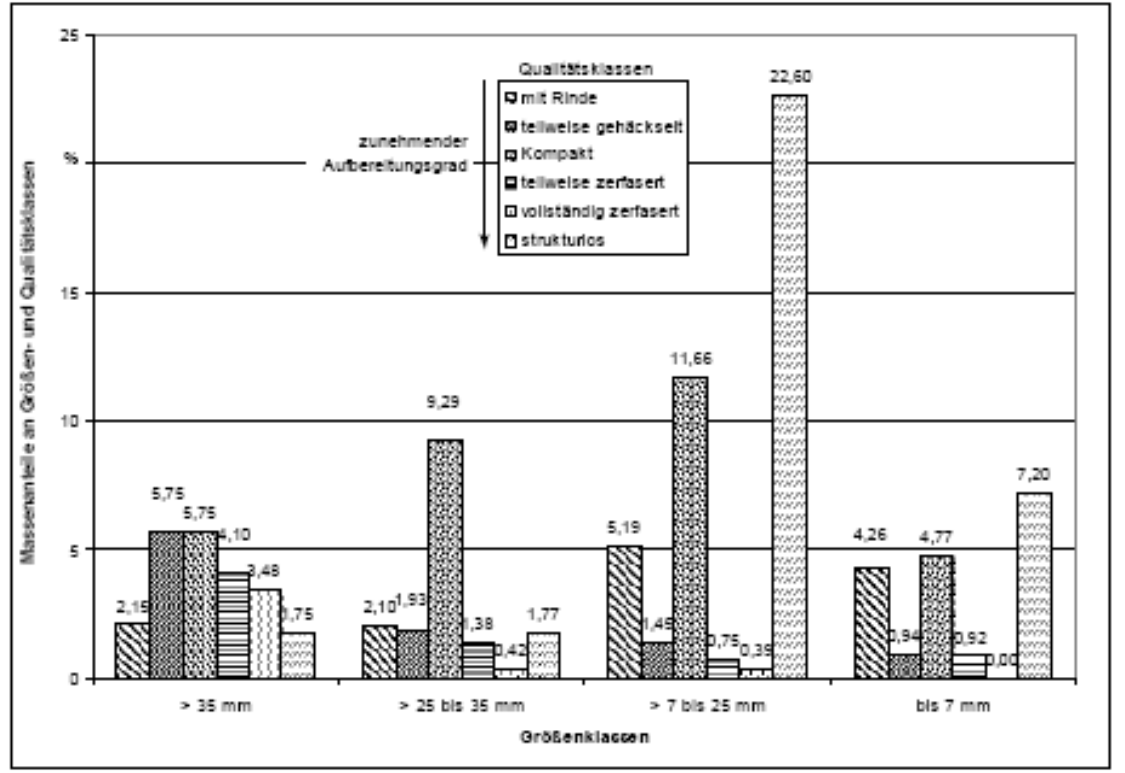

Abb. 24: Verteilung der Hackschnitzelqualitäten auf die definierten Größenklassen, Bearbeitungsmaschine: Tritucap 2; Bezugsgröße: Masse der Gesamtprobe (BLOCK, 2004)

Aussichtsreicherer Ergebnisse zu einer geeigneten Aufarbeitungsqualität zeigen dagegen Untersuchungen zum "Göttinger Gehölzmähhäckslers“, welcher zur Beerntung von Kurzumtriebsplantagen entwickelt wurde (WIENEKE, 1993). Das Maschinenkonzept arbeitet nach einem sehr ähnlichen Arbeits- und Aufarbeitungsprinzip mittels Schneckenhackertechnik und produzierte verwertbare und auch klassierbare Hackschnitzel (HARTMANN \& 
THUNEKE, 1997; GROTHAUS, 1993). Diese können zudem mittels Wurfgebläse geborgen werden, weshalb auch eine Adaption einer Überladetechnik an die Tritucap möglich sein sollte. Einschränkend muss allerdings angemerkt werden, dass die Einsätze des Göttinger Gehölzmähhäckslers nur in (baumartigen) Pappeln und Weiden in Reihenkultur und in einreihiger Bearbeitung erfolgten. Diese geben keinen Aufschluss über mögliche Arbeitsergebnisse in natürlich gewachsener Vegetation mit Bäumen und Büschen. Letztlich kann nur vermutet werden, dass das Aufarbeitungsprinzip eine Eignung zur Herstellung eines verwertbaren Häckselproduktes ermöglichen kann. Der Einzug der Triebe erfolgt auch bei diesem Maschinenkonzept senkrecht stehend. Dieses bedeutet, dass die Triebe in ihrer Ausrichtung nach dem Schneiden nicht verändert werden müssten. Dieses könnte vor allem in dichter Vegetation mit wenig Freiraum einen Bearbeitungsvorteil bedeuten, wie er z.B. in der Kurzumtriebsplantagenbeerntung festgestellt wurde (HARTMANN \& THUNEKE, 1997).

Aufgrund der Erfüllung bzw. der Aussicht auf eine Erfüllung wesentlicher Punkte des Basiskonzeptes wird im Rahmen der Vorentwicklung eine modifizierte Form der Tritucap zur Entbuschung von Offenflächen getestet. Dieses ist unter anderem auch damit begründet, dass eine baugleiche Version der in Brasilien eingesetzten Tritucap 2 zur Verfügung steht. Voraussetzung dafür ist allerdings eine Modifikation, um wesentliche Anforderungen wie die Materialbergung zu gewährleisten.

\subsection{Vorentwicklungen}

\subsubsection{Modifizierte Tritucap}

\subsubsection{Umsetzung des Maschinenkonzeptes Tritucap}

Die Tritucap muss für die vorliegenden Anforderungen modifiziert und dabei mit einer Überladeeinrichtung ausgerüstet werden. Die Komponenten zum Schneiden, Aufnehmen und Zuführen sowie zum Zerkleinern werden vorerst in ihrer Originalversion belassen. 


\subsubsection{1. Überladen}

Zur Erstellung einer Überladeeinrichtung werden die Erfahrungen aus dem selbstschneidenden Schneckenhackerkonzept, dem "Göttinger Gehölzmähhäcksler" zur Beerntung von Kurzumtriebsplantagen (GROTHAUS, 1993), genutzt und prinzipiell beide Konzepte miteinander kombiniert.

Zur Aufnahme des Häckselgutes von den Häckselrotoren wird direkt hinter dem Auswurfschacht ein erster Beschleunigerrotor mit einer Breite von $800 \mathrm{~mm}$ montiert. Dieser beschleunigt das von den Auswerfern beider Rotoren zugeförderte Material tangential mit vier Wurfleisten. Dabei lenkt er den Gutfluss von waagerechter Förderung auf die senkrechte um $90^{\circ}$ um. Beim „Göttinger Gehölzmähhäcksler" erfolgt die Umlenkung passiv ohne Förderelemente und neigt vor allem bei hohem Materialaufkommen zu Verstopfungen (WILHELM, 2004). Nach einer Förderstrecke von 1,8 m, in der das Gut auf eine Förderbreite von $30 \mathrm{~cm}$ konzentriert wird, folgt direkt vor dem Auswurfkrümmer ein zweiter Beschleuniger, welcher das Gut abermals beschleunigt und in den Auswurfkrümmer wirft. Dieser lenkt das Material in eine waagerechte Wurfrichtung um. Der Auswurfkrümmer ist $360^{\circ}$ hydraulisch drehbar, womit sich die Wurfrichtung einstellen lässt. Am Ende des Krümmers ist ein hydraulisch verstellbares Prallblech angebracht, mit dem das Material wiederum in einem um $90^{\circ}$ einstellbaren Winkel von der waagerechten in die senkrechte Wurfrichtung umstellen lässt, um einen entsprechenden Auffangbehälter von oben zu beladen.

Zur Bergung und zum Flächentransport des Häckselgutes wird bei den Einsätzen der Tritucap ein Frontladercontainer eingesetzt. Er besteht aus einem Blechaufsatz, der auf eine 2,2 m breite Standard-Erdschaufel aufgeschraubt ist. Das Fassungsvermögen beträgt 3 Kubikmeter, was bei frischen Hackschnitzeln bei einer Schüttdichte von ungefähr $300 \mathrm{~kg} / \mathrm{m}^{3}$ (Wassergehalt $\sim 40 \%$, nach KALTSCHMITT (2001)) einem ungefähren Beladungsgewicht von $900 \mathrm{~kg}$ entsprechen würde. Dazu kommt noch das Gewicht der Schaufel und des Aufsatzes mit fast $500 \mathrm{~kg}$, so dass sich ein Gewicht von $1400 \mathrm{~kg}$ im Frontlader ergeben könnte. Aus Gründen der Übersichtlichkeit, der Fahrstabilität und des zunehmenden Gesamtgewichtes 
wird diese Größe als ausreichend angenommen. Mit den Gehölzmasseaufkommen der Untersuchungen von RAHMANN (2000) in Beziehung gesetzt, ist bei diesem Behältervolumen mit ungefähr 11 Entladungsvorgängen bei einem Hektar entbuschter Fläche zu rechnen.

\subsection{Antriebskonzept}

Der Antrieb der Beschleuniger erfolgt mechanisch über Keilriemen, die mit der Hauptantriebswelle direkt vor dem T- Getriebe des Häckselrotorantriebes verbunden sind und über ein weiteres T-Getriebe zu den Beschleunigern verzweigt werden. Da die benötigten Drehzahlen der Beschleuniger sowohl bezüglich Masse- als auch Wurfleistung im Vorhinein nicht bekannt sind, wird im verzweigenden T-Getriebe eine Übersetzung von $1: 1,5$ gewählt, um auch bei niedrigen Antriebsdrehzahlen hohe Beschleunigerdrehzahlen zu erhalten.

\subsection{Mobileinheit}

Die Mobileinheit der modifizierten Tritucap mit Überladecontainer nach der Fertigstellung ist in Abb. 25 in einem ersten Versuch zu sehen.

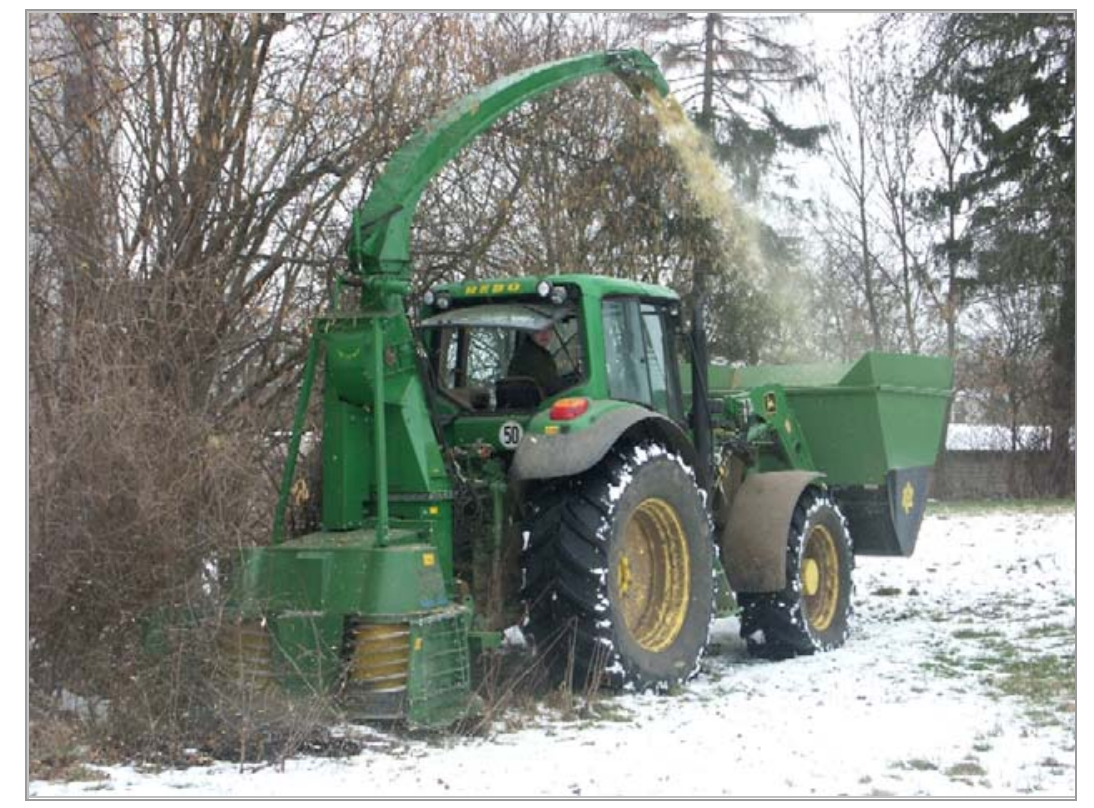

Abb. 25: Modifizierte Tritucap mit Überladeeinrichtung und Frontladercontainer bei vollmechanisierter Entbuschung 


\subsubsection{Praxiseinsätze der Tritucap}

Der Einsatz der Tritucap erfolgt auf den Versuchsflächen DB 2, DB 3 und der AFR, so dass das Maschinenkonzept sowohl in buschartiger als auch in baumartiger Vegetation in unterschiedlichen Vegetationsausprägungen getestet wird. Während der Einsätze erfolgen aufgrund der Versuchsergebnisse Umbauarbeiten und Weiterentwicklungen an dem Maschinenkonzept.

\subsection{Schneiden}

Die Sägeblätter der Tritucap trennen die Gehölzvegetation problemlos bis zu einem Durchmesser von $15 \mathrm{~cm}$. Über diesen Bereich treffen die Hölzer schon während des unvollendeten Schnittes auf die Auswerfer des Rotors, so dass diese gegen die noch nicht vollständig abgetrennten Triebe schlagen. Technisch ist bei diesem Durchmesser durch den speziellen Aufbau der maximale Schneiddurchmesser zum Abtrennen der Triebe erreicht. Insgesamt zeigen sich die Sägeblätter als sehr robust und nicht störanfällig gegenüber vertikal wirkenden Kräften wie aufstehenden Bäumen. Auch zwischen den Gehölzen stehendes Gras, ob vertrocknet oder in saftig grünem Zustand wird abgetrennt und bereitet im weiteren Arbeitsverlauf keine Probleme.

Die Arbeitsbreite der Tritucap mit ca. 2,1 $\mathrm{m}$ ist für den eingesetzten Schlepper zu gering. Dieser steht mit den Hinterreifen an jeder Seite ca. $15 \mathrm{~cm}$ über die Maschinenarbeitsbreite über, so dass über unbearbeitete Fläche gefahren und dort noch stehenden Gehölzen ausgewichen werden muss. Dieses verschärft sich bei Kurvenfahrt oder auch am Hang, da durch das Lenken ein größerer Fahrbereich benötigt wird. Das Durchfahren einer verbuschten Fläche ist so nicht möglich und bei Hangneigungen muss möglichst von unten her gearbeitet werden. Für den auftretenden Seitenschlupf am Hang steht dann ausreichend freie Fläche zur Verfügung.

Anhand der Praxiseinsätze kann die Form des Fanggestells, welches bei BLOCK (2004) im Brasilieneinsatz Verwendung fand, als nachteilig identifiziert werden (Abb. 16). Die Bügel des Fanggestells stehen in einer Höhe in die 
Bearbeitungsrichtung hinein, in der die Gehölze oftmals ineinander verwachsen und durch Dornen verhakt sind. Die Bügel können dort nicht zwischen den Gehölzen hindurchdringen und spannen diese vor dem Schneiden in Bearbeitungsrichtung vor, so dass sie anschließend nach dem Schnitt von der Maschine weggedrückt werden und nicht bearbeitet werden können.

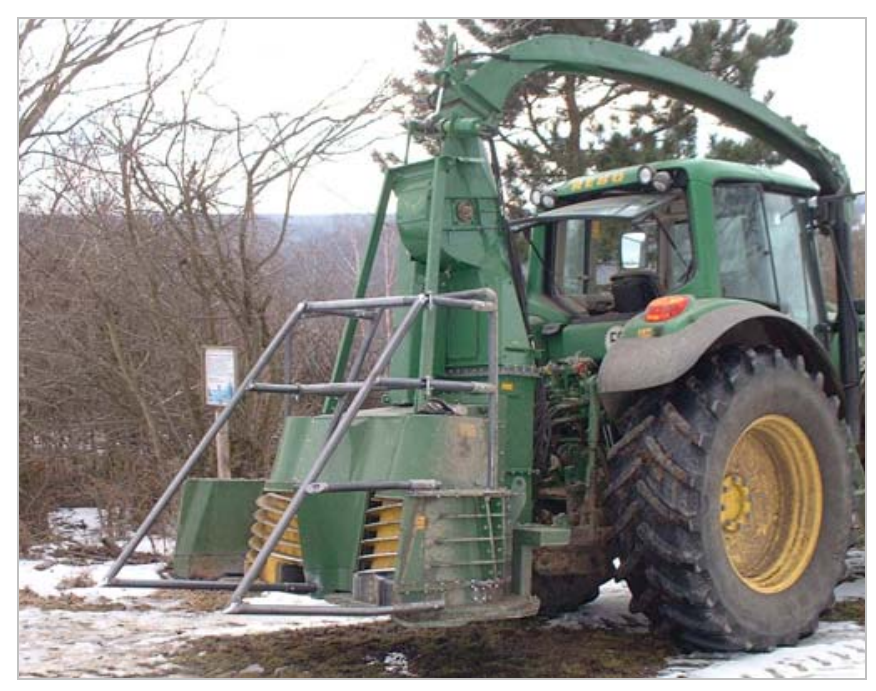

Abb. 26: Spitzbügel an Tritucap mit Überladeeinrichtung.

Der eingesetzte Spitzbügel (Abb. 26) teilt die Gehölze von der Stammbasis aus und kann sie entsprechend eine Kamms zufriedenstellend teilen. Die Höhe, welche aufgrund der teilweise in der Höhe voluminöser werdenden Kronen auf $2 \mathrm{~m}$ begrenzt ist, stellt sich bei Bäumen über ca. $4 \mathrm{~m}$ Länge jedoch als zu gering heraus, da diese durch den hohen Schwerpunkt vermehrt über den Bügel hinweg zum Schlepper kippen.

\subsection{Aufnehmen, Zuführen und Zerkleinern ${ }^{1}$}

Hinsichtlich der Gehölzbearbeitung eröffnen sich während der Ersteinsätze mehrere grundlegende Probleme am Maschinenkonzept. Das Arbeitsprinzip der Tritucap mit den senkrecht stehenden Häckselrotoren macht es zur Bedingung, dass die Haupttriebe stehend in den Häckselspalt gefördert

\footnotetext{
${ }^{1}$ Die Kapitel sind hier zusammengefasst, da sich die Vorgänge auf eine Komponente beziehen.
} 
werden, um durch einen idealen Schneckenschnitt zerkleinert zu werden (WIENEKE, 1993). Dazu müssen sie im Häckselspalt eingeklemmt werden (siehe Abb. 27). In der ursprünglichen Version der Tritucap erfolgt der tangentiale Transport der Triebe (1. in Abb. 27) ausschließlich über deren Reibung an dem Sägeblatt sowie dem Rotor und durch einen radialen Überstand der Auswerfer von $2 \mathrm{~cm}$ über die äußere Abmessung der Häckselschnecke hinaus.

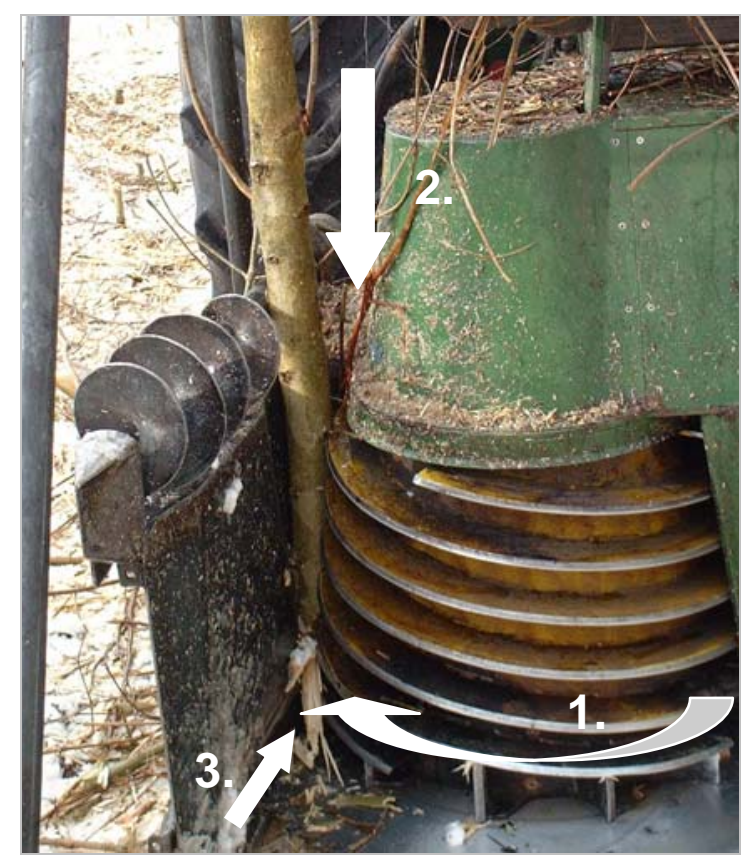

Abb. 27: Ideal geklemmter Trieb im Häckselspalt der Tritucap bei der Zerkleinerung; 1. tangentialer Transport zum Häckselspalt; 2. rotoraxialer Einzug zur Zerkleinerung; 3. Einzug der Triebachse annähernd parallel zum Pfeil bei waagerechtem Einzug der Zerkleinerung

Dieser tangentiale Transportmechanismus ist prinzipiell ausreichend, hat jedoch den Nachteil, dass die Auswerfer das Material sehr aggressiv in den unteren Bereich des Häckselspaltes ziehen. Die Folge ist, dass vor allem Triebe dünneren Durchmessers nicht hinreichend eingeklemmt werden können und sich ihre Triebachse im Häckselspalt in eine waagerechte Ausrichtung drehen. Sie erfahren durch die unter dem Häckselspalt (dem feststehenden Widerlager) in das Gehäuse einlaufenden Auswerfer ein Drehmoment. Die Konsequenz ist ein eher waagerechter Einzug der Triebe (Einzug der Triebachse parallel zu 3. in Abb. 27). Dieser erfolgt nicht mehr 
anhand der axialen Einzugsgeschwindigkeit des Häckselrotors sondern anhand dessen tangentialer Umfangsgeschwindigkeit, welche ungefähr das 8,4 -fache beträgt! Mehrere Meter lange Triebe werden so in einem Bruchteil einer Sekunde mit großer Wucht in den Häckselspalt gezogen. Einerseits treten dadurch sehr hohe und plötzliche Spitzen im Leistungsbedarf auf und andererseits ist der typische Schneckenschnitt nicht mehr gegeben. Die Zerkleinerung erfolgt zu großen Teilen durch die Auswerfer und die Schnittlänge wird vornehmlich durch den Abstand der Auswerfer zueinander bestimmt. Im Hackgut entstehen deshalb Hackstücklängen bzw. Triebabschnitte von bis zu $60 \mathrm{~cm}$ Länge, was ungefähr dem Abstand der Auswerfer am Umfang entspricht. Der gleiche Fall tritt auf, wenn die tangentiale Zuführung in den Häckselspalt nur ungenügend zeitnah oder gar nicht erfolgt. Die frei stehenden Triebe bekommen das Übergewicht und fallen nach dem Schneiden um. Anschließend könnten sie nur waagerecht liegend aufgenommen werden oder es muss über sie unbearbeitet hinweg gefahren werden. Die Tritucap lässt durch ihr spezielles Einzugsverfahren bzw. das Fehlen einer technischen Ausrüstung zum Aufrichten von Trieben keine Möglichkeit zu, waagerecht liegendes Material aufzunehmen und dieses anschließend ideal mit dem Schneckenschnitt zu zerkleinern.

Die beobachteten Tendenzen zum waagerechten Einzug sind in Abb. 28 entsprechend der Vegetationsbedingungen abgebildet. Sie stammen aus der Beobachtung des Aufnahmeverhaltens sowie aus der Auswertung der Störfalldokumentation. 


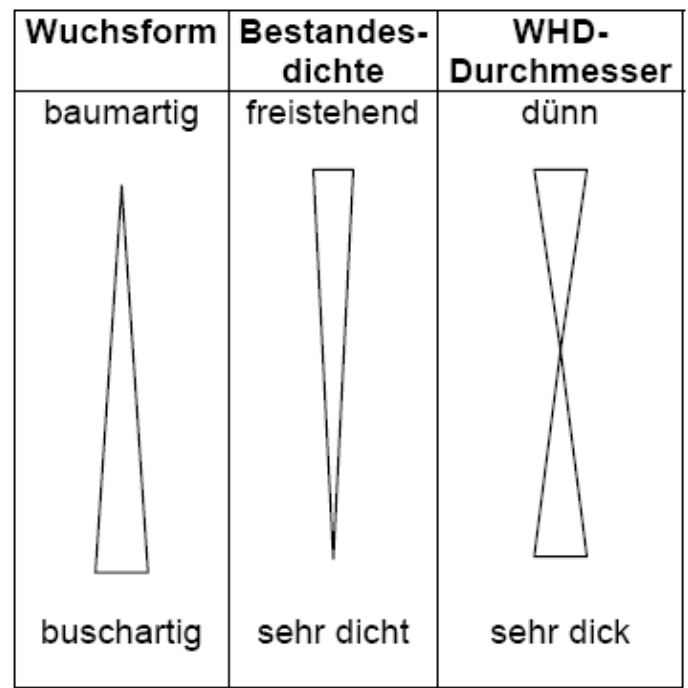

Abb. 28: Beobachtete Tendenz zum waagerechten Einzug der Triebe beim Maschinenkonzept der Tritucap in Abhängigkeit von Vegetationsbedingungen (Breite entspricht Größe der Tendenz)

Hinsichtlich der Wuchsform wird das Auftreten des waagerechten Einzuges bei buschartigen Gehölzen begünstigt. Diese bestehen häufig aus mehreren sich am Boden verzweigenden Trieben, welche sich durch ihren Wuchs von der Schnittstelle teils stark (bis $\sim 45^{\circ}$ ) wegneigen (Abb. 29).

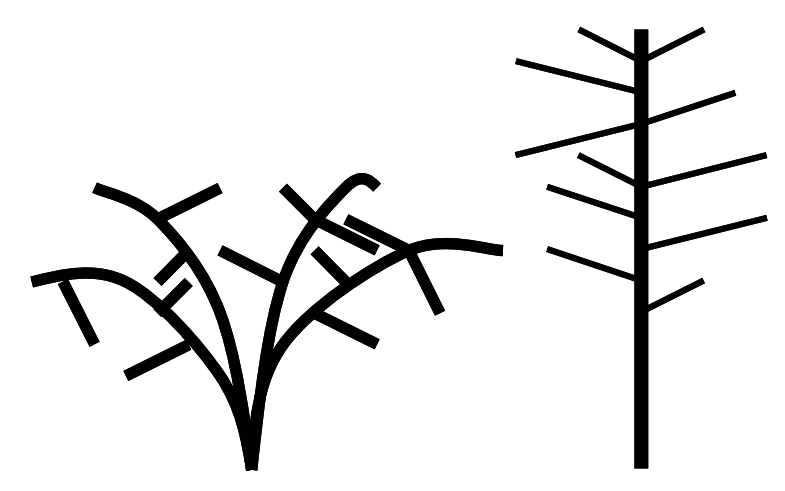

Abb. 29: Typische Wuchsformen von Gehölzen; links: buschartig; rechts: baumartig

Da sie alle relativ zeitgleich am Wurzelstock geschnitten werden, aber nicht gleichzeitig geklemmt oder gehalten werden können, fallen vor allem die seitlich und in Bearbeitungsrichtung geneigten Triebe um, bevor sie bearbeitet werde können. In dichter werdenden Beständen verringert sich die Tendenz zum Umfallen, vermutlich da sich die Triebe gegenseitig stützen. 
Hinsichtlich des WHD kann vor allem im sehr dünnen und sehr dicken Bereich das Umfallen bzw. der waagerechte Einzug beobachtet werden. Der Grund für einen auffällig häufigen waagerechten Einzug der dünnen Triebe wird oben schon aufgrund der aggressiv wirkenden Auswerfer gegenüber der geringen Klemmwirkung genannt. Dieses erfolgt somit eher beim Häckselprozess. Bei zunehmend dickeren WHD scheint die Trägheit der größeren Triebmasse die Förderung zum Häckselspalt zu hemmen, da sie auffällig länger unbewegt auf dem Sägeblatt stehen bleiben. Dieses gibt die notwendige Zeit zum Umfallen vor einem tangentialen Transport und einer sicheren Klemmung. Des weiteren besitzen dickere Triebe in der Regel massivere Seitenäste im Maschinenbereich. Diese stützen sich auf den Maschinenaufbauten ab, weßhalb sie nach dem Schneiden wieder aus dem Einzugsbereich gedrückt werden und sie anschließend in Fahrtrichtung umfallen können. Aufgrund der zunehmend voluminöseren Gestalt der dickerer Büsche, fällt dieses bei Büschen dickeren Ausmaßes besonders auf. Hinsichtlich der Bestandesdichte und auch dem Auftreten mehrerer Triebe pro Wurzelstock zeigen buschartige Gehölze einen weiteren Schwachpunkt des Häckselkonzeptes auf. Die Triebe sind oft derart in sich verwachsen oder durch Dornen verhakt, dass sie entweder nicht einzeln vom Häckselrotor zugeführt werden können, da sie an anderen noch stehenden festhängen, oder dass ein einzelner beim Einzug mehrere Triebe mit sich zieht. Eine kontinuierlich gleichmäßige Materialzufuhr ist so nicht gegeben und diese ist vom Bediener ausschließlich durch das Fahrverhalten nur gering beeinflussbar. Eine gleichzeitige Zuführung mehrerer Triebe führt oft zu einer Überlastung aufgrund eines zu plötzlichen, hohen Materialaufkommens.

In der Regel bewirkt der waagerechte Einzug der Triebe eine Störung mit Blockade der Maschine. Bei größerem Materialaufkommen (einzelne Triebe in größerem Durchmesserbereich ab ca. $4 \mathrm{~cm}$ oder auch mehrere mit geringerem Durchmesser) entsteht diese durch einen zu hohen und plötzlich auftretenden Leistungsbedarf. Dieser kann sich durch das Auslösen der Überlastsicherung an der Gelenkwelle oder sogar durch das Absterben des 
Schleppers äußern. Die dabei auftretenden Blockaden sind vornehmlich auf den Häckselspalt lokalisiert (Abb. 30).

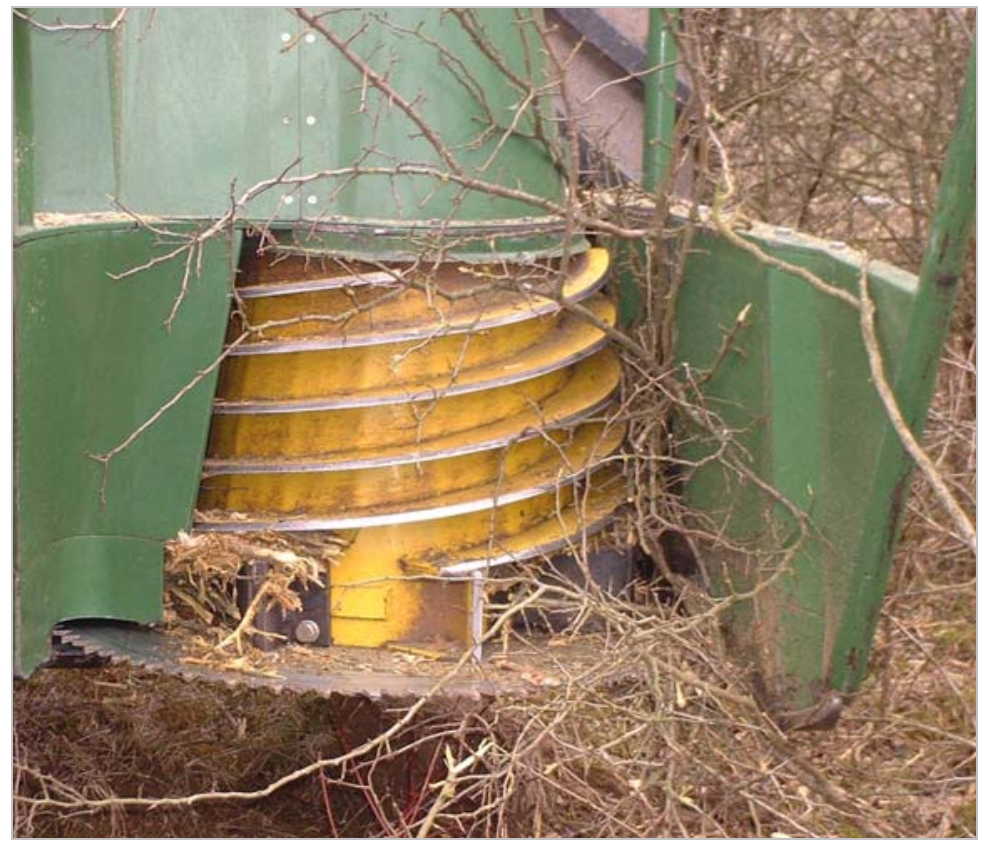

Abb. 30: Verstopfung des Häckselspaltes durch verstärkt waagerecht eingezogenes Material dünneren Durchmessers

Erfolgen die Blockaden nicht am Häckselspalt, so führt das überlange Hackmaterial zum Stillstand des ersten Beschleunigers oder durch Querstellen zum Verstopfen des Förderweges .

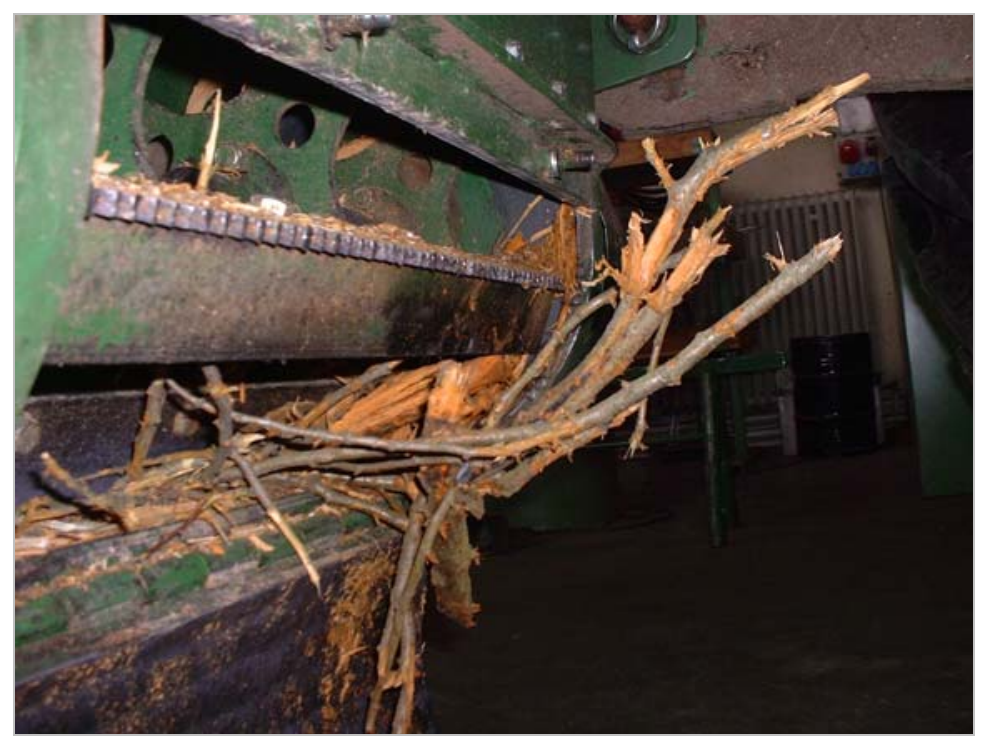

Abb. 31: Geöffnete Wartungsklappe am ersten Beschleuniger der Tritucap nach Blockade durch dünnes und überlanges Gehölzmaterial nach waagerechtem Triebeinzug durch die Häckselrotoren 
Um das Einzugsproblem zu lösen, werden zwei Wegen verfolgt. Einerseits soll das Umfallen der Triebe und deren senkrechte Zuführung garantiert werden und andererseits unabhängig von der Zuführposition das Auftreten von Überlängen verhindert werden.

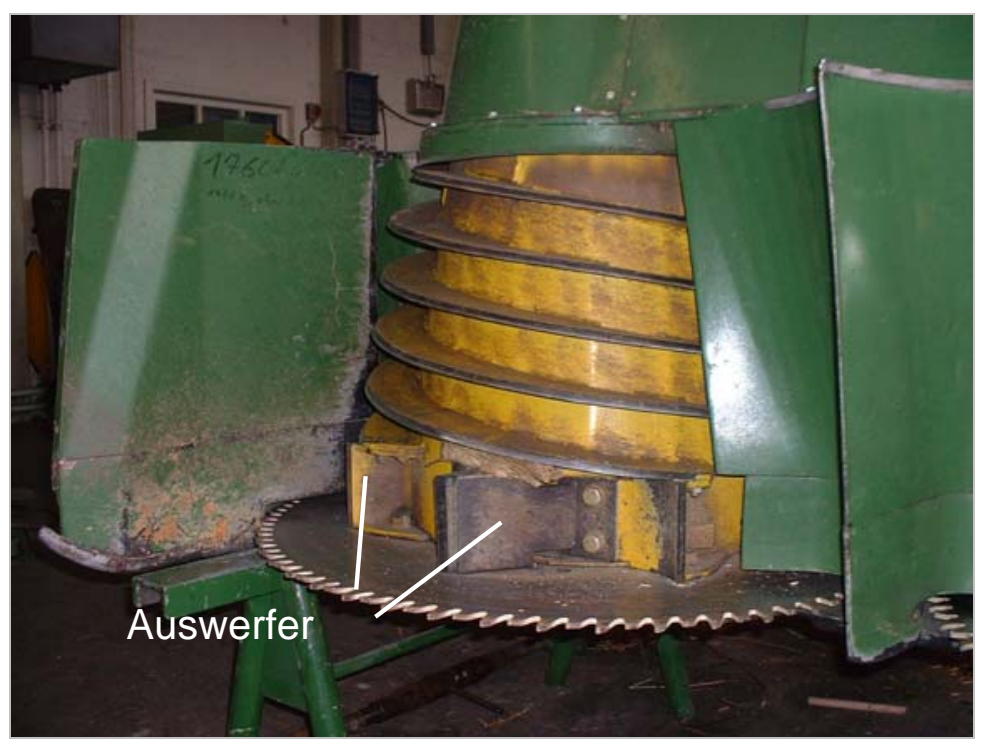

Abb. 32: Rechter Häckselrotor der modifizierten Tritucap 3 mit insgesamt acht Auswerfern pro Häckselrotor

Als einfache Ansätze zur Verhinderung der für den Überladevorgang und letztlich auch für eine spätere Verwertung nachteiligen Überlängen werden

1. am Rotor zusätzliche Auswerfer integriert (Abb. 32) und

2. ein Auswerfermantel mit einer Mehrzahl kurzer Auswerfer eingezogen (Abb. 33).

Beide haben zum Ziel das waagerecht eingezogene Material in ausreichend kürzere Hackstücke zu zerkleinern, wenn sie den auch nicht durch den Schneckenschnitt zerkleinert werden. Der Mantel soll durch den geringeren Abstand zum Gehäuse den Transport von zu dickem Material behindern und erst nach entsprechender Aufarbeitung einen Durchlass zum Beschleuniger zulassen. 


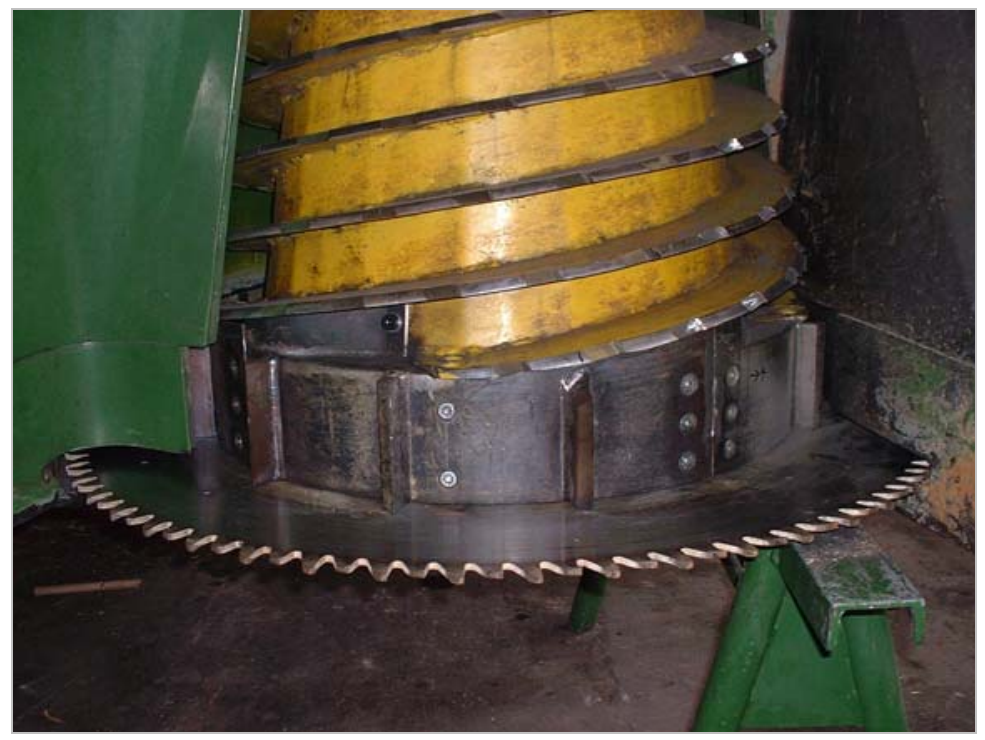

Abb. 33: Linker Häckselrotor der modifizierten Tritucap 3 mit Auswerfermantel

Die angestrebte Wirkung hinsichtlich der Hackstücklänge ist zwar in gewissem Umfang gegeben, so dass Blockaden des Beschleunigers nur noch selten vorkommen, jedoch zeigen die Auswerfer ein derartig aggressives Einzugsverhalten, dass sie auch dickere Triebe, deren Klemmung im Häckselspalt vorher geleistet werden konnte, waagerecht eingezogen werden. Die Folge ist entsprecht eine zunehmende Blockadehäufigkeit am Häckselrotor, weshalb diese Varianten der Problemlösung aufgegeben werden. 


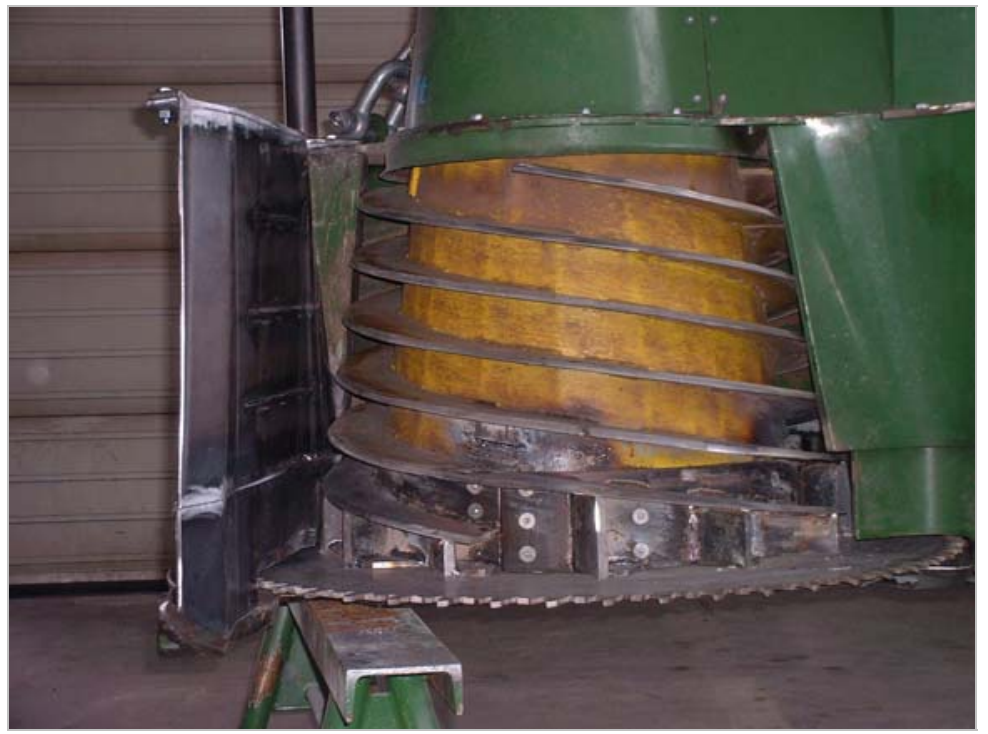

Abb. 34: Umbau eines Häckselrotors an der Tritucap:

Verlängerung der Schneidwindungen und Integration von drei Auswerfern je Schneidwindung ohne Überstand über die Schneide; Anpassung der Seitenwand und des Häckselspaltes.

Ein weiterer Ansatz zur Lösung des Problems besteht im zielgerichteten Zuführen der Triebe bei gleichzeitiger Verringerung des tangentialen Zuführvermögens von Trieben durch die Auswerfer in den Häckselspalt. Einerseits soll auf diese Art eine vorrangig senkrecht stehende Zuführung der Triebe erreicht werden und andererseits das Drehmoment auf die Triebe durch die Auswerfer im Häckselspalt verringert werden.

Zu diesem Zweck wird die Häckselrotorvariante mit den Windungsverlängerungen eingebaut (Abb. 34) und, soweit sie im Rahmen der Maschinenkonstruktion baulich integrierbar sind, Fördereinrichtungen angebracht.

Die Verlängerungen der Schneiden sollen einen möglichst weiten Schnittbereich der Schneckenschneide bis annähernd auf das Sägeblattniveau garantieren. Die bis hinter die Schneidenkante verkürzten Auswerfer sollen bei einem ideal eingezogenen und geklemmten Trieb erst auf das Holz treffen, wenn dieses im Querschnitt des Triebes durchtrennt ist. Dieses soll die tangential wirkenden Einzugskräfte im Auswerferbereich und das daraus resultierende Drehmoment zum Auslösen des Kippens der Triebe vermindern. Waagerecht eingezogene Triebe sollen zu einem gewissen Teil, 
wenn auch schräg zu Stammachse, von der nun tiefer reichenden Schneckenschneide zerkleinert werden, da deren ziehender Schnitt einen geringeren Leistungsbedarf versprach (TSCHEUSCHNER, 2004). Der weitere Teil der Zerkleinerung waagerechter Triebe wäre weiterhin durch die eingekürzten Auswerfer gegeben. Durch deren Einkürzung ist jedoch auch eine verminderte tangentiale Transportleistung in den Häckselspalt zu erwarten.

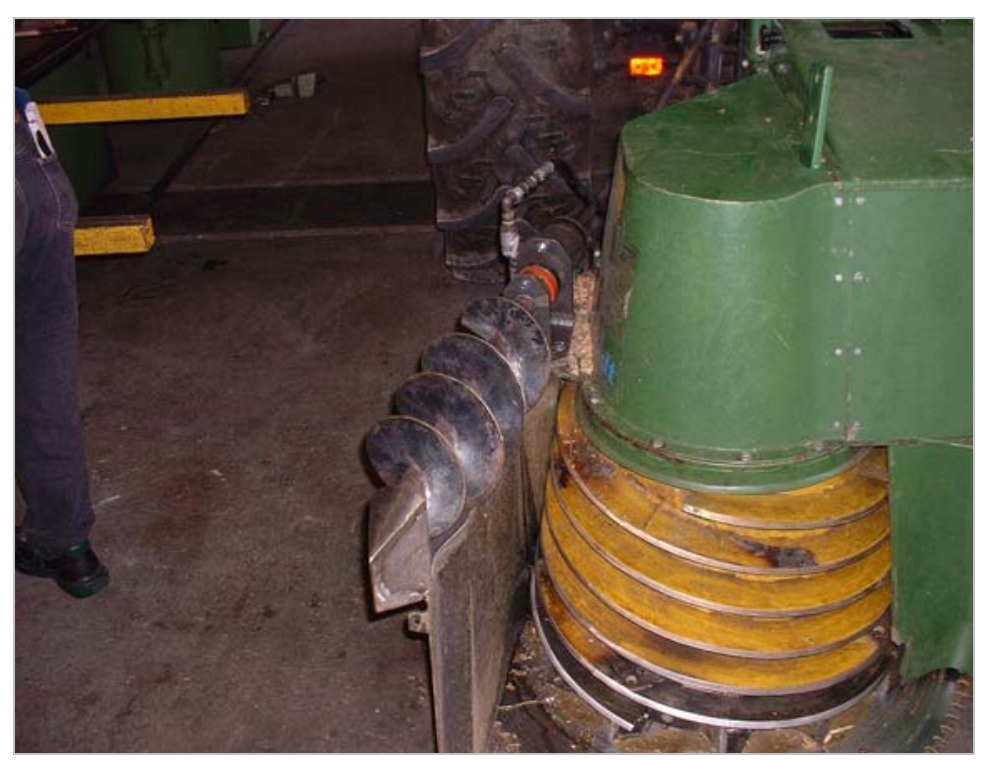

Abb. 35: Auf Seitenwand der Tritucap installierte Förderschnecke (hydrostatisch angetrieben)

Um den Transport der Triebe in den Häckselspalt zu verbessern, wird am Häckselspalt auf der Seitenwand eine hydraulisch angetriebene Förderschnecke (Abb. 35) und auf der Maschinenmitte der hydraulisch angetriebene Förderstern angebracht (Abb. 36). 


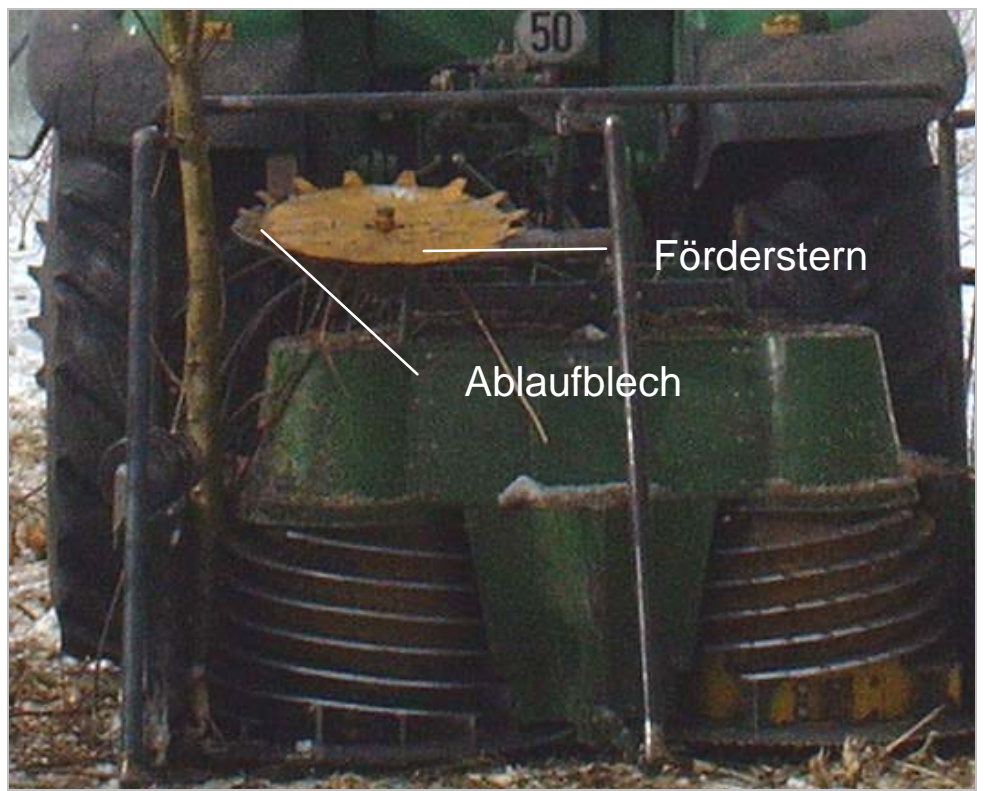

Abb. 36: Einseitig montierter Förderstern auf Tritucap zur Unterstützung der Zuführung der Triebe zum Häckselspalt.

Der Förderstern soll den zügigen tangentialen Transport nach dem Schnitt von der Maschinenmitte aus unterstützen und die Förderschnecke eine gleichmäßigere Zufuhr der Triebe sowie deren senkrechte Ausrichtung im Häckselspalt garantieren. Dabei wird der Fördervorgang des Sterns durch ein Ablaufblech in einer einstellbaren Position unterbrochen. Ein direktes Halten der Triebe in Arbeitsrichtung kann mit dem reinen Förderstern ohne ein Gegenlager nicht erreicht werden (MARTIN ET AL., 2008). Baulich stellen sie die einzigen möglichen Varianten von Fördereinrichtungen dar, die an diesem Maschinenkonzept zu realisieren sind. Großvolumige Förderwalzen, wie sie bei Hakkila (1989) bei Mähhäckslerprototypen im Forstbereich erprobt wurden, lassen sich aufgrund des begrenzten Bauraumes zwischen Schneid- und Häckseleinheit nicht verwirklichen. Haspelsysteme, wie sie an Mähdreschern zu finden sind, könnten nur von oben in den Bestand eingreifen, was aufgrund der vorliegenden Höhen von $>4 \mathrm{~m}$ nicht zu realisieren ist.

Um einen Einsatzvergleich offensichtlicher zu machen, wird der Umbau des Rotors mit Windungsverlängerungen sowie die Installation der Fördereinrichtungen nur auf einer Maschinenseite durchgeführt und der andere in der Standardversion des Brasilieneinsatzes belassen. 
Bei Versuchseinsätzen auf der Versuchsfläche AFR in fast ausschließlich baumartigen Bestand kann eine erhebliche Verbesserung durch den Umbau beobachtet werden. Ein vergleichender Versuch erfolgt auf zwei Flächenstreifen von jeweils $3 \times 30 \mathrm{~m}\left(90 \mathrm{~m}^{2}\right)$, die von freistehenden Bäumen in einen sehr dichten Baumbestand übergehen. Jeder Streifen wird jeweils nur mit einem Rotor, dem Standardrotor bzw. dem modifizierten, bearbeitet. Bei dem modifizierten Rotor kann nur eine Blockade festgestellt werden. Diese erfolgt bei einem dicken Trieb von $10 \mathrm{~cm}$ WHD, der waagerecht eingezogen wird. Insgesamt sind nur wenige, vor allem dünne Triebe zu beobachten, die waagerecht eingezogen werden, da sie von dem Förderstern nicht hinreichend erfasst werden. Im weiteren Förderweg bereiten sie jedoch keine Probleme. Der Standardrotor dagegen weist acht Blockaden durch waagerecht eingezogene Gehölze auf, die bei dünnen als auch bei dicken Trieben und vor allem im frei- bis engstehendem Bestand (dort sechs mal) auftreten. Dieses entspricht den vorherigen Beobachtungen, dass die Tendenz zum waagerechten Einzug durch eine geringere Bestandesdichte vergrößert wird.

Bei dem Einsatz in verschiedenster buschartiger Vegetation auf der Versuchsfläche DB 2 kann dagegen keine ausreichende Verbesserung festgestellt werden. Weder eine ausreichende Förderung aller Triebe stehend zum Häckselspalt noch eine dosiertere und kontrollierbarere Zuführung in den Häckselspalt kann mit Hilfe der Fördereinrichtungen realisiert werden. Der modifizierte Rotor zeigt zwar eine geringere Blockadeneigung in dünnem Material (2 bis $6 \mathrm{~cm}$ ), jedoch führt ein zunehmend dickerer WHD sowie auch eine zunehmende Zahl an Trieben pro Wurzelstock zu einem vermehrten Blockadeaufkommen. Vornehmlich ineinander verwachsene Triebe können nicht vereinzelt bzw. dosiert zugeführt werden (Abb. 37). 


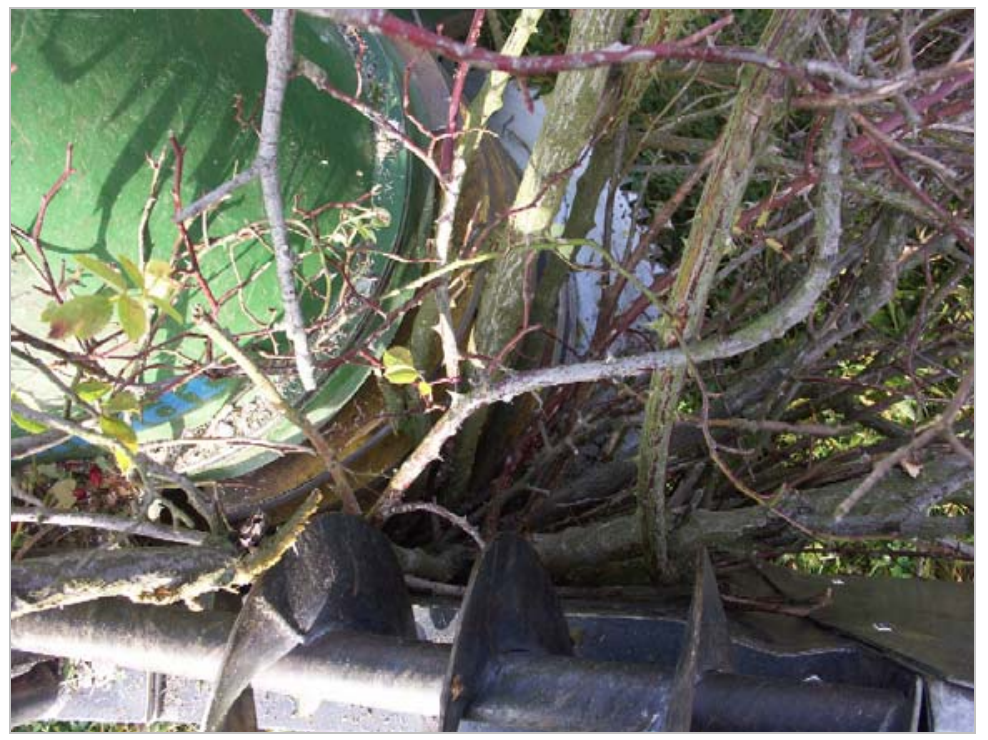

Abb. 37: Blockade des Häckselrotors mit verlängerten Windungen durch den gleichzeitigen Einzug einer mehrtriebigen Hundsrose (Blick von oben in den Häckselspalt)

Nach der Durchführung der Entbuschungsversuche können an der Tritucap keine technischen Lösungen am Häckselaggregat oder der Zuführung des Gehölzmaterials ermittelt werden, welche eine erfolgsversprechende Lösung zur Bearbeitung der Vegetation darstellen können. Aus diesem Grund werden die weiteren Arbeiten an der modifizierten Tritucap eingestellt.

\subsection{Mobileinheit}

Die Mobileinheit aus Schlepper, der relativ kurz angebauten Tritucap in der Drei- Punkt- Hydraulik und dem Frontladercontainer zeichnet sich durch eine hohe Flexibilität auf der Fläche aus. Das Rangieren und Anfahren der Gehölzvegetation ist durch die relativ kurze Mobileinheit sehr gut möglich. Positiv wirkt sich auch der Frontladercontainer aus, da er zur Verkürzung der Gesamtmaschine ausgehoben werden kann, um über Hindernisse bei Kurvenfahrt hinweggesetzt werden zu können. Die Gesamtübersichtlichkeit zur Beurteilung der Fahrverhältnisse ist durch den mittig positionieren Überladekrümmer auf der Tritucap in Arbeitsrichtung und durch die Rückwand des Überladecontainers zwar eingeschränkt, jedoch reicht das verbleibende Sichtfeld ausreichend aus, um die Fahrsituation zu beurteilen. 
Mit dem Überladekrümmer kann der Frontladercontainer sehr zielsicher über den Schlepper hinweg und ohne nennenswerte Verluste beladen werden. Durch den weiten Einstellbereich der Wurfrichtung des Krümmers ist eine umfangreich situationsangepasste Positionierung des Containers möglich.

Der Arbeitsbereich der Sägeblätter und der Häckselaggregate kann vom Fahrerstand aus nicht direkt eingesehen werden, was dazu führt, dass der Arbeitszustand der Häckselschnecken und der Sägeblätter nur sekundär (Geräuschentwicklung, etc.) und die Arbeitshöhe nur aufgrund der Maschinenhöhe hinter der Maschine mit der Erfahrung des Fahrers beurteilt werden können. Dieses stellt ein grundsätzliches Problem dar, da die Position des Fahrers bei einer Anbaumaschine in Schubfahrt hinter dem Arbeitsbereich angeordnet ist. Aufgrund der Erfahrung des Bedieners ist es jedoch fortwährend möglich die Gehölze wenige Zentimeter über dem Boden abzutrennen. Das Einstellen der genauen Schnitthöhe wird bei der alleinigen Führung durch die Drei-Punkt- Hydraulik jedoch zusätzlich durch Höhenschwankungen, hervorgerufen durch Unebenheiten bei der Überfahrt, erschwert. Die Höhenführung über die Drei- Punkt- Hydraulik stellt sich in diesem Einsatzgebiet deshalb auch nur als bedingt geeignet heraus. Auch Block (2004) berichtet von diesen Erfahrungen und empfiehlt eine visuelle Einstellhilfe oder gar eine Höhenautomatik zur eigenständigen Einstellung durch das Fahrzeug.

\subsubsection{Evaluation der Tritucap}

Die Anforderung zum vollemechanisierten Entfernen der Gehölzvegetation mit nur einem Bediener auf dem Schlepper und eine anschließende Verladung von aufgearbeitetem Gehölzmaterial kann prinzipiell bestätigt werden. Das vollständige Abtrennen kann sowohl in busch- als auch baumartiger Vegetation im angestrebten Einsatzbereich mit den Sägeblättern erreicht werden. Durch den Arbeitsplatz in der Kabine eines modernen landwirtschaftlichen Schleppers ist dabei für den Bediener ein umfangreich komfortables Arbeitsumfeld gegeben und bietet auch einen entsprechenden Schutz bei der Bearbeitung. Eine umfangreiche Bewertung des 
Arbeitsvorganges durch den Bediener ist aufgrund der eingeschränkten Übersichtlichkeit des Arbeitsbereiches jedoch nur unzureichend möglich.

Das Maschinenkonzept selbst kann die technischen Anforderungen der Anforderungsliste vor allem hinsichtlich der sicheren Aufnahme und Übergabe an die Aufarbeitung nicht erfüllen, um einen annähernd blockadefreien Arbeitsablauf zu gewährleisten. Dieser Mangel ist besonders in der zu bearbeitenden buschartigen Vegetation ausgeprägt. Die Vegetations- und Arbeitsbedingungen und entsprechend die Anforderungen an das Arbeitsprinzip müssen bei den Einsätzen in Brasilien abweichend von den hier vorgefundenen sein. Sicherlich kommt in dieser Anwendung zu den Vegetationsanforderungen an das Maschinenkonzept noch die Bergung des Hackmaterials hinzu. Ein betriebssicherer Einsatz im Zielbereich der Bearbeitung scheitert jedoch schon durch das bestehende Zerkleinerungsaggregat. Der spezielle Aufbau dieser Form des Schneckenhackers mit dem ebenso speziellen Anspruch der eher senkrecht stehenden Triebachsen in der Zuführung kann in diesem Einsatzbereich so nur unzureichend sicher gestellt werden. Die anschließend auftretenden Probleme mit der Verladetechnik am Beschleuniger können dabei als sekundärer Effekt angesehen werden. Bei einer entsprechenden Aufarbeitungsqualität des Hackgutes wären diese nicht oder nur vermindert aufgetreten.

Da trotz der vielen Blockaden und der Arbeiten im Überlastbereich der Gelenkwelle keine nennenswerten Schäden an der Maschine entstehen, kann das Maschinenkonzept selbst als sehr robust eingestuft werden. Weder an den Sägeblättern noch an den Häckselschnecken oder dem Gehäuse können Materialermüdungen festgestellt werden.

Hinsichtlich der Einsatzauswirkungen des Verfahrens auf Flora, Fauna und Boden liegen zu diesem Zeitpunkt der Entwicklung kaum Ergebnisse vor. Augenscheinlich sind bisher keine Schädigungen feststellbar, die über einen in der Landwirtschaft üblichen Schleppereinsatz nach guter fachlicher Praxis hinausgehen. Befahrungsmuster auf dem Boden sind zwar zu erkennen, jedoch zeigen diese keine Spurausbildung, so dass hier von plastischen 
Verformungen nicht ausgegangen werden muss (BRENNDÖRFER, 1995). Positiv dazu trägt jedoch auch bei, dass bei den Versuchen fast ausschließlich in der vegetationsfreien Zeit bei frostigen bzw. trockenen Bodenverhältnissen gearbeitet wird.

\subsubsection{Zwischenfazit zur modifizierten Tritucap}

Grundsätzlich zeichnet sich bei den Arbeiten auf den Versuchsflächen ab, dass eine vollmechanisierte Entbuschung nach dem Verfahrenskonzept landbzw. forstwirtschaftlicher Schlepper-Anbaumaschine bei gleichzeitiger Materialbergung ein Erfolg versprechendes Verfahren darstellt. Die Anforderungen zum Entfernen der Gehölzvegetation mit nur einem Bediener auf dem Schlepper und eine anschließende Verladung von aufgearbeitetem Gehölzmaterial kann prinzipiell bestätigt werden.

Der Einsatz des modifizierten Tritucap- Häckslers in der vollmechanisierten Landschaftspflege zeigt jedoch die Möglichkeiten und Grenzen dieses Maschinenkonzeptes auf.

Da bei der Entbuschung von sukzessiv gewachsener Gehölzvegetation der Anspruch der senkrechten stehenden Zuführung der Triebe technisch weder erfüllbar scheint noch deren Auswirkungen auf den weiteren Arbeitsprozess technisch verhindert werden können, muss die Verwendung dieses Maschinenkonzeptes in der vollmechanisierten Entbuschung bei gleichzeitiger Materialbergung als ungeeignet eingestuft werden. Sicherlich zeigt die technische Bearbeitung des Maschinenkonzeptes auch Wirkungen, die die Arbeitsqualität verbessern. Diese sind jedoch vornehmlich auf baumartige Gehölze beschränkt. Dieses gibt letztlich auch einen Hinweis darauf, dass das Zerkleinerungsprinzip für gerade gewachsene Gehölzbestände mit einem Haupttrieb bei der Kurzumtriebsbeerntung von Pappeln und Weiden entwickelt wurde. Für den hier angestrebten Einsatzbereich ist es nicht anwendbar.

Das Verfahrenskonzept von Schlepper-Anbaumaschine und getragenem Container zur Bergung des Materials erscheint insgesamt zielführend zu 
sein. Es wird aus diesem Grund mit einem überarbeiteten Maschinenkonzept weitergeführt.

\subsubsection{Gehölzmähhäcksler 05}

\subsubsection{Konzeptentwicklung zum GMH 05}

Da die Anforderungsliste des Entbuschungsverfahrens in Kernpunkten mit dem Maschinenkonzept der modifizierten Tritucap nicht erfüllbar sind, muss ein neues Maschinenkonzept erarbeitet werden.

Das Verfahrenskonzept der schlepperbasierten Mähernte wird in diesem Zusammenhang weiter verfolgt, da die Einsatzergebnisse der Tritucap bei einem ausreichend funktionsfähigen Maschinenkonzept auf das Erreichen wesentlicher Verfahrensanforderungen schließen lassen. Von Maschinenseite aus lässt sich die Überladetechnik des Wurfgebläses sowie das Trennen der Gehölze mittels Sägeblättern gut in das Konzept integrieren. Auch die Bergung des Hackmaterials mit dem Frontladercontainer und dem Überladen von der Maschine am Schlepperheck über den Schlepper hinweg kann in dieser Form wieder in das Konzept integriert werden. Kernpunkte sind das Aufnehmen und Zerkleinern, welche einer technisch- konzeptionellen Lösung bedürfen.

Da ein Umfallen der Triebe in eine waagerechtere Position vor allem bei buschartigen Gehölzen nicht ausreichend verhindert werden kann, muss dieses zu dem Schluss führen, dass dieses in den Aufnahmeprozess eingearbeitet werden muss. Ein selektives Wiederaufrichten erscheint zu aufwendig und eine gleichzeitige Aufnahme von stark voneinander abweichenden Triebachsen wäre nicht möglich, da jedes Häckselverfahren zur optimalen Zerkleinerung ein Mindestmaß an gleichmäßig und möglichst parallel ausgerichteter Haupttriebachsen einfordert (BRUSCHE, 1983; WiENEKE \& DÖHRER, 1994; FIRUS \& BELTER, 1995A).

Gehölztriebe besitzen eher zur Spitze geneigte Seitenäste und lassen sich bei parallel zur Stammachse und dabei zur Spitze zeigender Äste am Besten kompaktieren. Eine Zuführung zu einem Häckselaggregat erfolgt deshalb vorzugsweise von der Basis (der Schnittfläche) aus. Die Gehölze müssen so 
nicht „gegen den Strich“ zugeführt werden, was das Einführen erleichtert (BRUSCHE, 1983).

Insgesamt bedeutet dies im Zusammenhang mit dem Umfallen der Triebe nach dem Schneiden, dass die Aufnahme der Triebe in einer eher waagerechten Position erfolgen muss und letztlich auch, dass alle Triebe nach dem Schneiden eine annähernd parallel zueinander ausgerichtete Position einnehmen sollten. Anschließend müssen die Triebe in dieser Position vom Häckselaggregat aufgenommen und zerkleinert werden.

Dieses lässt sich nur ereichen, indem der Bereich des Schneidens räumlich und baulich von dem Bereich des Häckselns getrennt wird. Zwischen den Komponenten muss ein entsprechender Raum für Zuführkomponenten und die Ausrichtung der Triebe verbleiben. Die Zuführkomponenten müssen einerseits die abgetrennten Gehölze über eine gewisse Maschinenbreite erfassen, komprimieren und entsprechend vorbereitet dem Zerkleinerungsaggregat zuführen. Ähnliche Grundkonzepte von mähenden Holzerntemaschinen werden bei HAKKILA (1989) als selbstfahrende Prototypen beschrieben, die sich im Forstbereich der skandinavischen Länder jedoch nicht durchsetzen konnten. Des weiteren arbeiten im weitesten Sinne die selbstfahrenden Vollernter auf Maishäckslerbasis in der Kurzumtriebsplantagenbeerntung nach diesem System. Das Schneiden und waagerechte Aufnehmen ist dort auf den Erntevorsatz beschränkt und das Häckselaggregat in der Basismaschine integriert. Die Vorsätze sind aber ausschließlich in Reihenkulturen einsetzbar und besitzen nur eine eingeschränkte Schnittbreite (ScHOLZ, 2007; Abb. 38). 


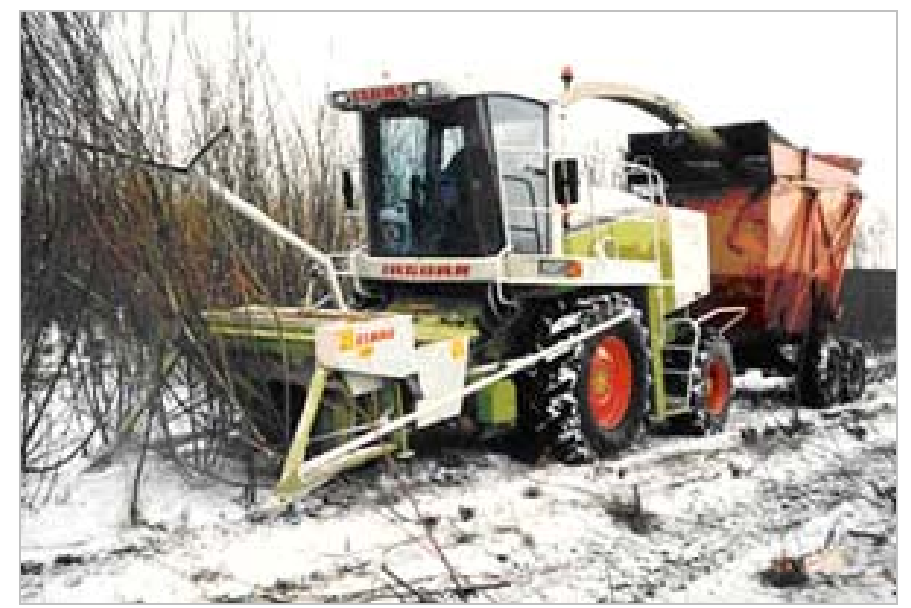

Abb. 38: Feldhäcksler mit Erntevorsatz für Kurzumtriebsplantagen (Quelle: Wippermann, Hamburg)

In der Kurzumtriebsplantagenbeerntung werden die Gehölze mit einem Druckbügel vor dem Schneiden in Fahrtrichtung gedrückt und vorgespannt. Dieses stellt einerseits die waagerechte Ausrichtung sicher und hat den Effekt einer Schleuderbewegung in Richtung des Zuführschachtes sobald der Trieb durchtrennt ist (HARTMANN \& THUNEKE, 1997).

Durch eine vor den Zuführ- und Zerkleinerungsaggregaten positionierte Schneideinrichtung und der Verwendung eines Druckbügels könnte dieser Effekt auch bei der Entbuschung eingesetzt werden. Zudem könnte der Druckbügel derart gestaltet sein, dass er die Gehölze vor dem Schneiden vorkonzentriert. Das Vorspannen der Triebe vor dem Schneiden hat in der Plantagenbeerntung eine ungewollte Erhöhung des Spleiß- und Bruchverhaltens am Wurzelstock zur Folge (HARTMANN \& THUNEKE, 1997). Dieses könnte bei der Entbuschung wiederum von Vorteil sein, um die Wiederaustriebskraft der Sukzessionsvegetation zu hemmen (DILLENBURGER, 2005).

Die Zuführaggregate müssen robust sein und ein Zusammenführen, ein Klemmen und ein Fördern bewirken können. FIRUS \& BELTER (1998) zeigen ein System aus senkrecht stehenden Förderwalzen auf, welches bei der Aufnahme von Reisig und Restholz als vorzüglich ermittelt wurde. Es ähnelt den Zuführaggregaten der mähenden Holzerntemaschinenprototypen von 
HAKKILA (1989) und scheint aufgrund des dortigen Einsatzgebietes auch in dieser Vegetation einsetzbar.

Durch die waagerecht angestrebte Einzugsrichtung kann das Schneckenhackersystem in seiner ursprünglichen von Brusche (1983) beschriebenen Form mit gleichermaßen waagerecht ausgerichteten Häckselrotoren verwendet werden. Der Anspruch an die Ausrichtung der Triebe unterscheidet sich beim waagerecht ausgerichteten Häckselrotor eines Schneckenhackers nur unwesentlich von den anderen Zerkleinerungstechniken wie Trommel- oder Scheibenradhacker (siehe BRUSCHE, 1983). Die angestrebten Vorzüge der Schneckenhackerzerkleinerung müssen somit nach den Erfahrungen mit der Tritucap nicht verworfen werden. Lediglich die Einzugsgeschwindigkeit sollte in diesem System verringert werden, um den Leistungsbedarf bei den Arbeiten abzusenken. Dieses ist durch eine geringere Schneckensteigung möglich (NURMI, 1986). Um weiterhin eine Produktion von Grobhackgut zu ermöglichen, sollte dieses durch eine geringere Windungsanzahl bei einem der Tritucap gleichen Windungsabstand von $80 \mathrm{~mm}$ erreicht werden. Nach den Ergebnissen von NuRMı (1986) kann dieses zwar den spezifischen Energiebedarf der Zerkleinerung erhöhen, führt aber grundsätzlich zu einem verringerten Leistungsbedarf während der Zerkleinerung. Um das Gehölzmaterial über die Gesamtschnittbreite nicht vollständig komprimieren zu müssen und den Förderweg über die Maschinenbreite niedrig zu halten, sollte die Zerkleinerung wie bei der Tritucap auf zwei Häckselrotoren verteilt werden.

Die Einzugsgeschwindigkeit kann auch im Bezug auf die Bearbeitungsleistung der bei diesem Konzept liegende Schneckenhäckslerrotoren erniedrigt werden. Die Tritucap erlaubt nur eine gleichzeitige Bearbeitung eines bzw. sehr weniger dünner Triebe, weshalb dort eine hohe Einzugsgeschwindigkeit zwingend notwendig ist, um eine ausreichende Arbeitsleistung zu erreichen. Im Prinzip müssen die Triebe nacheinander bearbeitet werden. Eine geringere Einzugsgeschwindigkeit eines Standardschneckenhackers müsste eine vergleichbare Arbeitsleistung 
zulassen, da dort durch eine andere Art der Zuführung die Möglichkeit zur gleichzeitigen Bearbeitung einer höheren Triebzahl besteht, welche den Effekt auf die geringere Einzugsgeschwindigkeit wieder ausgleichen könnte. Dieses würde zwar letztlich durch die Zunahme der Gesamtschnittfläche wieder zu einem Anstieg des Leistungsbedarfs führen (BRUSCHE, 1983), dabei ließe sich der Leistungsbedarf aber der Bearbeitungssituation angepasst über die Zuführmenge steuern.

\subsubsection{Umsetzung des Maschinenkonzeptes GMH 05}

Das aus diesen Überlegungen völlig neu entwickelte Konzept eines selbstschneidenden Schneckenhäckslers ist der Gehölzmähhäcksler 05 (GMH05), der im Rahmen der weiteren Entwicklungsarbeit eingesetzt wird. Er ist in Abb. 39 und Abb. 40 dargestellt.

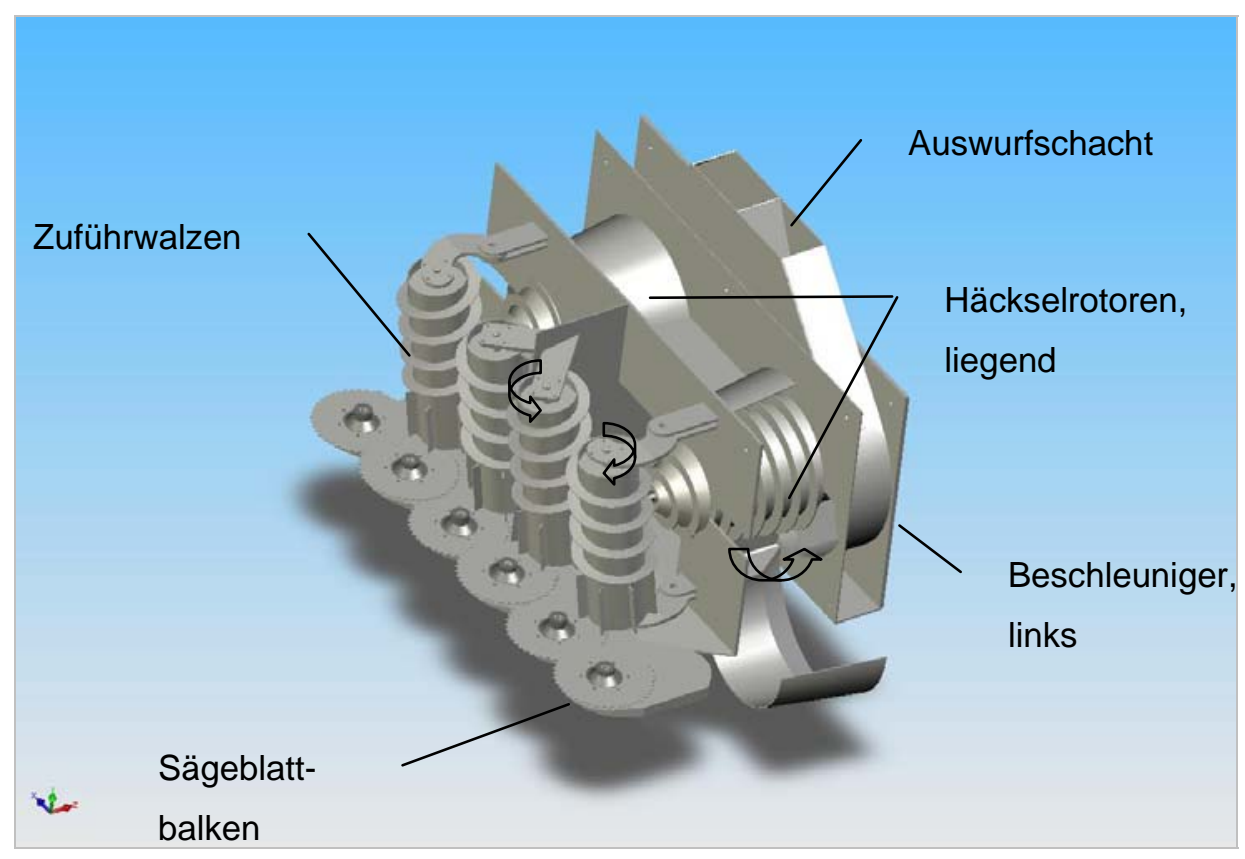

Abb. 39: Konstruktionsprinzip des Gehölzmähhäckslers 05 (GMH05); Richtung der Blockpfeile entspricht der Drehrichtung

Wesentliche Unterschiede zur Tritucap sind die waagerecht ausgerichteten Achsen der Häckselrotoren (liegende Häckselrotoren) und die Trennung der Schneid- und Häckselkomponenten zu zwei örtlich getrennten Aggregaten. Durch den dort abgebildeten Maschinenaufbau erreicht der GMH 05 größere bauliche Abmaße. Die größte Breite mit 2,4 m ist durch den Sägeblattbalken gegeben. Die Länge der Maschine beträgt von den Unterlenkeraufnahmen 
bis zur äußeren Sägeblattkante 2,6 $\mathrm{m}$ und das Gewicht 2,6 t. Der Maschinenschwerpunkt liegt im Bereich der Häckselrotoren in einem Abstand von 1,4 m von den Unterlenkeraufnahmen entfernt.

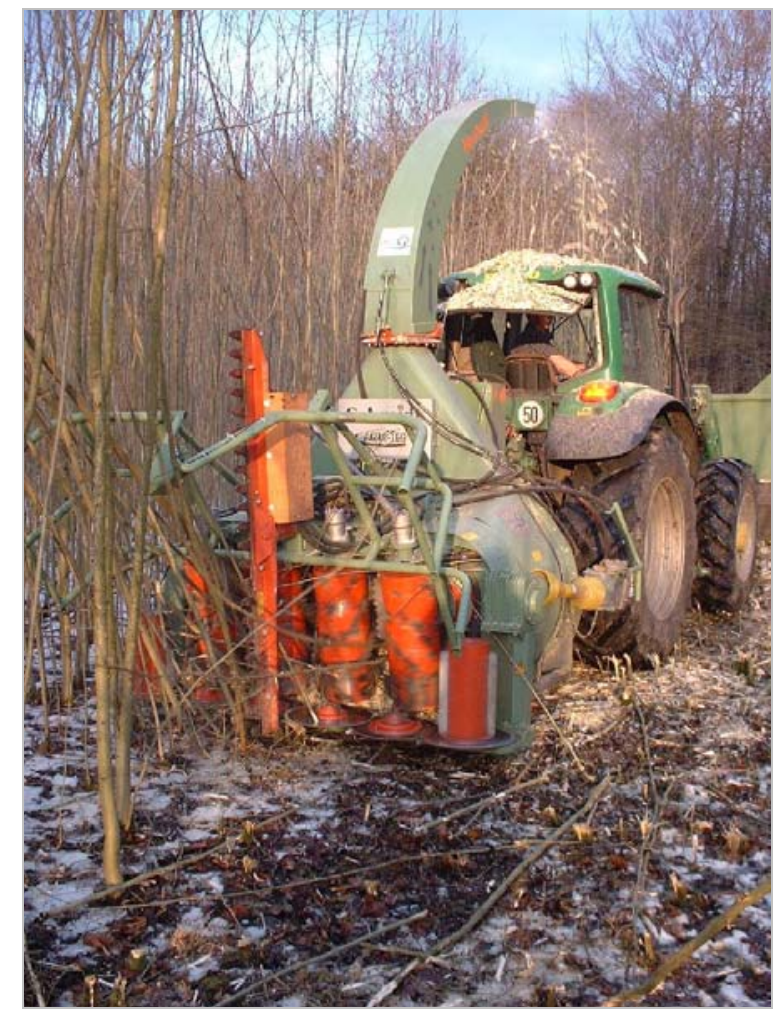

Abb. 40: Gehölzmähhäcksler 05 im Entbuschungseinsatz

\subsection{Schneiden}

Beim GMH05 wird die stehende Gehölzvegetation mit einem separaten Sägeblattbalken abgetrennt. Dieser besteht aus einer modifizierten Schneidgarnitur eines stabilen Tellermähwerkes. Auf dem Sägeblattbalken sind statt der Mähteller sechs aneinander gereihte Sägeblätter mit einem Durchmesser von $470 \mathrm{~mm}$ montiert, wobei diese abwechselnd um 70 bzw. $95 \mathrm{~mm}$ überlappen. Um den Kontakt zwischen den Sägeblättern auch bei senkrecht wirkenden Kräften zu verhindern, wird zwischen den Überlappungen der Sägeblätter ein Höhenabstand von $22 \mathrm{~mm}$ eingehalten. Nach Schilderung des Einsatzzweckes wurde die Zahnform KV (Wolfszahn) bei $Z=32$ Zähnen von der konstruierenden Fa. Felde, Remscheid (FELDE, 2005) empfohlen. Durch die Materialstärke zuzüglich der Schränkung resultiert daraus eine Schnittbreite von $8 \mathrm{~mm}$. 
Unter den Sägeblättern ist eine Getriebeeinheit angeordnet, in der die Lagerung sowie auch der Stirnradantrieb untergebracht ist. Der Antrieb hat eine Höhe von $70 \mathrm{~mm}$ und trägt dementsprechend diese Höhe von der Maschinenunterseite bis zur Sägeblattunterseite auf. Um im Schneidprozess nicht mit der vollen Höhe des Getriebes gegen die Stümpfe zu stoßen, wird der Balken leicht in Fahrtrichtung geneigt eingebaut, so dass die Schnitthöhe mit dem Getriebekasten überlappt. Dieses kann nur bis zu einem gewissen Winkel geschehen, da die Schnittrichtung der Sägeblätter sonst zu stark von der Fahrtrichtung abweicht und zusätzliche Vertikalkräfte auf das Sägeblatt wirken. Auf den äußeren Sägeblättern sind sogenannte Förderzylinder angebracht. Sie sollen das Material, welches vom äußeren Sägeblatt geschnitten wird, zur Maschinenmitte befördern. Sie bestehen aus einem Zylinder mit vier radial ausgerichteten Flachstählen, welche zentrisch auf der Lagerung des jeweils äußeren Sägeblattes angebracht sind (siehe Abb. 41). Aufgrund ihrer Positionierung und des Antriebes sollen sie hier beim Schneiden aufgeführt werden, obwohl sie eigentlich zu den Förderelementen zählen.

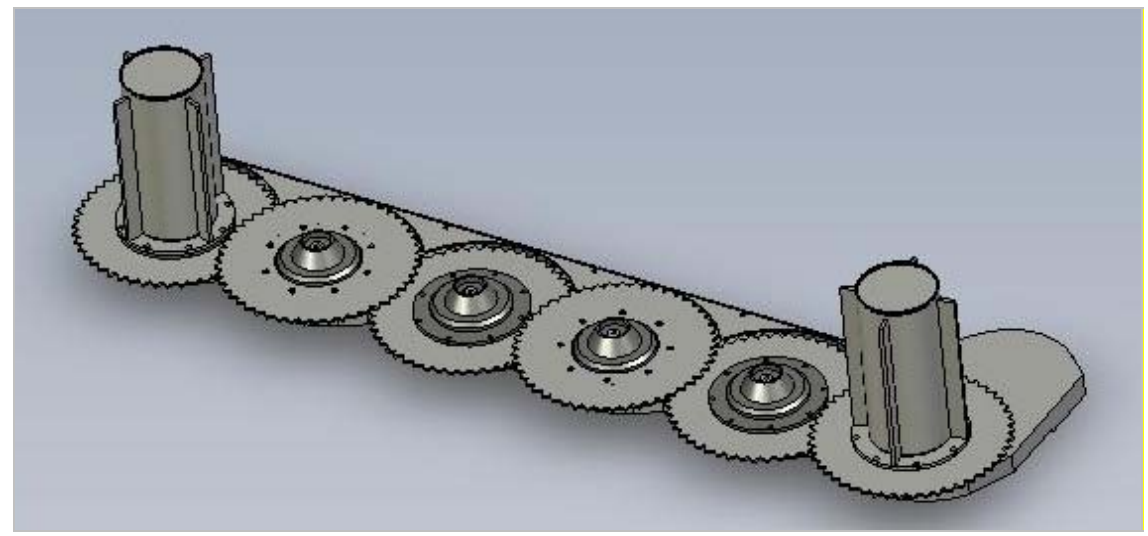

Abb. 41: Sägeblattbalken des Gehölzmähhäckslers 05 mit Förderzylindern auf jeweils äußerem Sägeblatt

Über dem Sägeblattbalken ist ein Drückbügel angebracht (Abb. 40). Er ist über den Zuführwalzen gelagert und kann in Arbeitsrichtung geschwenkt werden. Er steht an den Seiten sowie in der Mitte in die Bearbeitungsrichtung vor und bildet somit zwei Gabeln. Aufgerichtet erreicht der Bügel von der Sägeblattoberseite aus eine Höhe von 2,2 m. 


\subsection{Aufnehmen und Zuführen}

Zwischen dem Sägeblattbalken und der Häckseleinheit des GMH 05 sind Zuführaggregate in Form von senkrecht stehenden Zuführwalzen installiert. Das rechte ${ }^{2}$ und das linke Paar der Zuführwalzen fördert das abgeschnittene Gehölzmaterial jeweils auf die dahinter liegende Häckselschnecke. Sie werden über einen permanenten Ölfluss vom Schlepper aus hydrostatisch betrieben. Die jeweils äußeren Zuführwalzen können federbelastet wegschwenken, um Platz für das zwischen den Walzen hindurch zu transportierende Gehölzmaterial zu schaffen. In der Grundversion sind sie alle mit glatten Schneckenwendeln sowie im unteren Bereich mit jeweils acht radial zur Zylindermitte ausgerichteten Flachstählen bestückt. Die Drehung der Zuführwalzen führt zu einer senkrecht zum Maschinenboden gerichteten Förderbewegung der Schneckenwendeln. Die Flachstähle führten eine tangential wirkende Förderbewegung zu den Häckselschnecken aus.

Zwischen den zwei Zuführwalzenpaaren wird eine stabile, hydraulisch angetriebene Astschere der Fa. Dücker installiert (siehe Abb. 40). Sie arbeitet nach dem Prinzip des linearen Scherenschnitts und konnte bei einer Schnittfrequenz von ca. 80 Schnitten/s Triebstärken von bis zu $50 \mathrm{~mm}$ durchtrennen. Die Schere ist senkrecht stehend direkt vor den Sägeblättern positioniert. Der Schnittbereich reicht von der Schnitthöhe der Sägeblätter bis zu 2,2 m darüber und dient der Materialaufteilung der abgetrennten Gehölze in zwei Gutströme, welche jeweils auf einen Häckselrotor geleitet werden.

\subsection{Zerkleinern}

Der GMH 05 ist mit zwei nebeneinander liegenden Schneckenhäckslerrotoren ausgestattet. Die Häckselrotoren sind eingängig mit einem Windungsabstand von $80 \mathrm{~mm}$ und einem Konuswinkel von $10^{\circ}$ ausgeführt. Ihr größter Durchmesser beträgt $700 \mathrm{~mm}$. Theoretisch beträgt die

\footnotetext{
${ }^{2}$ Positionsangaben an der Maschine sind, wenn nicht näher erläutert, immer aus der Draufsicht in Arbeitsrichtung (Fahrtrichtung) der Maschine angegeben (Sägeblattbalken befindet sich z.B. vorne).
} 
Einzugsgeschwindigkeit bei einer Zapfwellendrehzahl von $1000 \mathrm{U} / \mathrm{Min}$ $1,33 \mathrm{~m} / \mathrm{s}$.

Am Häckselaggregat werden die Gehölztriebe unter die Häckselschnecke gefördert, wo ein halbrund ausgeformter Boden mit einem unter der Häckselrotorachse befindlicher Anschlag als Widerlager den sich verjüngenden Häckselspalt bildet. In Abb. 42 ist ein Blick zwischen dem Zuführwalzenpaar hindurch in die rechte Häckseleinheit dargestellt. Sobald die Schneiden der Häckselschnecken in die Gehölze einschneiden, werden diese durch den Zerkleinerungsvorgang selbsttätig weiter zugeführt (Selbsteinzug).

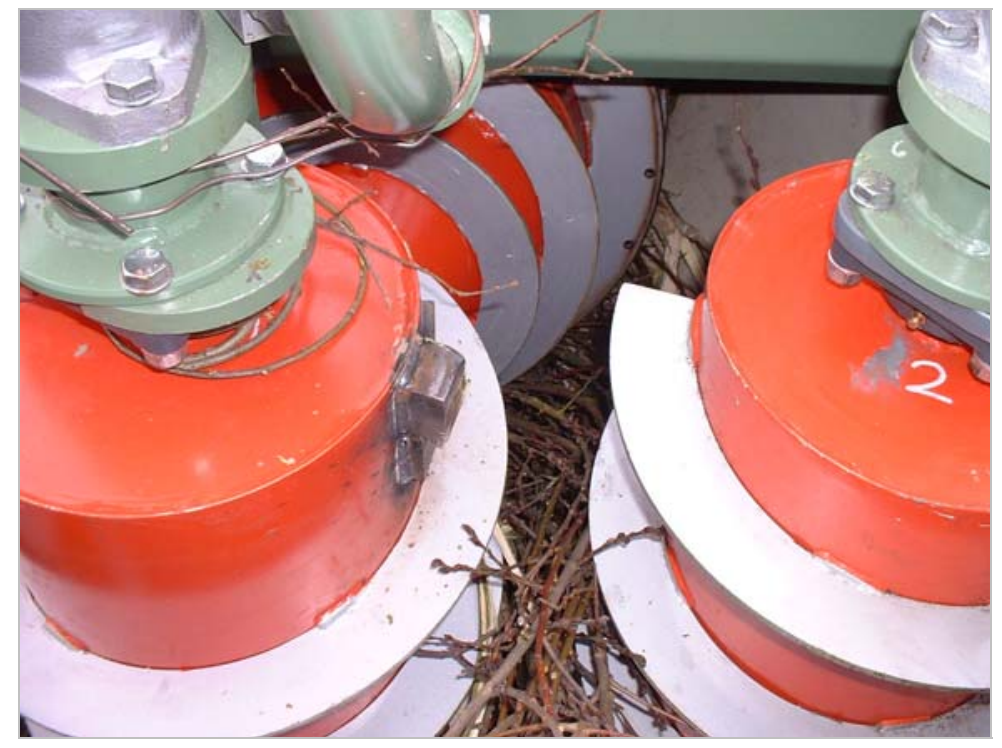

Abb. 42: Blick in die rechte Häckseleinheit des Gehölzmähhäckslers 05 zwischen dem Zuführwalzenpaar hindurch

Nach der Zerkleinerung fallen die entstandenen Hackstücke in den jeweiligen Beschleuniger der Häckselrotors.

\subsubsection{4. Ü̈berladen}

Die Beschleuniger zum Auswurf des Häckselmaterials werden weiterhin an der Basis der Häckselrotoren belassen und sind fest mit diesen verbunden. Aufgrund der waagrechten Ausrichtung der Häckselrotoren und somit auch der Drehachsen der Beschleuniger können die senkrechte Förderung des Hackmaterials ohne eine größere Umlenkung erfolgen. Die Beschleuniger 
besitzen jeweils vier Auswurfschaufeln und haben einen Durchmesser von $900 \mathrm{~mm}$. Sie werfen das Häckselmaterial jeweils in einen Auswurfschacht, die in einem maschinenmittig angeordneten Trichter zusammenlaufen, auf dem der Auswurfkrümmer angebracht ist. Der Auswurfkrümmer ist um $360^{\circ}$ hydraulisch drehbar und besitzt ein hydraulisch verstellbares Prallblech am Ende, um den Strahl des Häckselgutes in einem wählbaren Bereich von bis zu $90^{\circ}$ von einer waagerechten in eine senkrechte Wurfrichtung umzulenken. So kann auch hier ein Auffangbehälter von oben zielgerichtet beladen werden.

\subsection{Antriebskonzept}

Durch die Trennung der Schneidkomponenten von den Häckselaggregaten sowie deren waagerechter Ausrichtung wird ein neues Antriebskonzept notwendig. Es ist schematisch in Abb. 43 abgebildet und weit komplexer als bei der Tritucap.

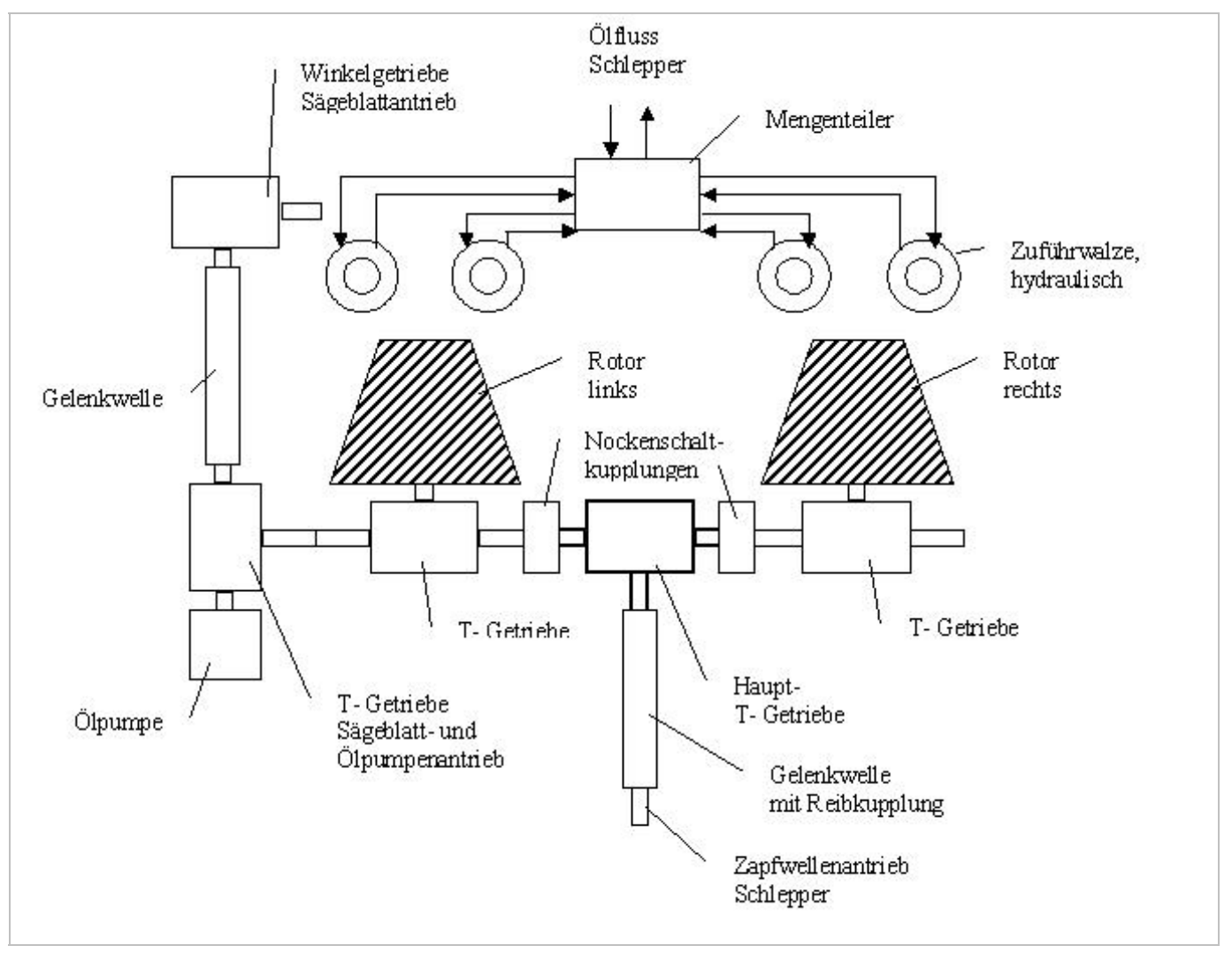

Abb. 43: Antriebskonzept des Gehölzmähhäckslers 05

(schematisch) 
Der Hauptantrieb des GMH 05 erfolgt mechanisch über den Zapfwellenanschluss des Schleppers und verzweigt sich über ein T-Getriebe auf ein jeweiliges T-Getriebe an jedem Häckselrotor. Die Übersetzung ist hier bei allen Getrieben $1: 1$, so dass die Drehzahl der Schlepperzapfwelle anliegt. Ausgelegt ist das Maschinenkonzept auf eine Schlepperausgangsdrehzahl von $1000 \mathrm{U} / \mathrm{min}$, da das Drehmoment der übertragenden Schlepperleistung somit geringer gehalten werden kann (HAKKILA, 1989). Zur Sicherung des Antriebsstranges vor Beschädigungen wird zwischen Schlepper und Maschinenhaupt- T- Getriebe eine Gelenkwelle mit einer Reibkupplung eingesetzt, welche ein Lösemoment von 3500 Nm besitzt. Zum Schutz vor schädlichen Lastwechseln zwischen den Häckselrotoren (vergl. BLOCK, 2004) werden zwischen dem Haupt- T-Getriebe und den T-Getrieben der Häckselrotoren Nockenschaltkupplungen installiert, die analog zur Tritucap entgegen der Drehrichtung eingebaut werden.

Am T-Getriebe des linken Häckselrotors wird der Antrieb des Sägeblattbalkens weitergeführt. Das folgende T- Getriebe zwischen Ölpumpe und der Gelenkwelle zum Winkelgetriebe des Sägeblattantriebes untersetzt die Drehzahl mit dem Verhältnis 1,92:1, um auf die erforderliche Eingangsdrehzahl am Winkelgetriebe des Sägeblattantriebes (Übersetzung $1: 1)$ zu gelangen. Die Drehzahl beträgt hier $521 \mathrm{U} / \mathrm{min}$ bei einer Schlepperausgangsdrehzahl von $1000 \mathrm{U} / \mathrm{min}$. Die Nebenantriebe am Stirnradgetriebe des Sägeblattbalkens, auf denen die Sägeblätter montiert sind, übersetzen diese wiederum nach dem Verhältnis $1: 4,5$, woraus eine Drehzahl der Kreissägeblätter von 2344,5 U/ min resultiert. Daraus ergibt sich eine Umfangsgeschwindigkeit von $57,7 \mathrm{~m} / \mathrm{s}$, welche vom Hersteller der Sägeblätter (Fa. Felde, Remscheid) als „gut ausreichend“ befunden wird.

Der Antrieb der Zuführwalzen erfolgt hydrostatisch über ein einstellbares Zweikreis- Proportionalsteuerventil vom Schlepper aus, welches die Ölmotoren über Mengenteiler kontinuierlich mit Drucköl versorgt. So kann die gleichmäßige Drehzahl der vier Zuführwalzen stufenlos vom Schlepper aus eingestellt werden. Die Ölpumpe am T-Getriebe zum Sägeblattantrieb 
versorgt die Astschere mit Drucköl. Dieses wird in einem zusätzlichen Öltank (80I Fassungsvermögen, mit Ölfilter und Überdruckventil) mitgeführt, welcher zwischen dem Schlepper und dem GMH 05 über der Oberlenkerhalterung angebracht wird. Dabei werden biologisch abbaubare Hydrauliköle eingesetzt, die ein Gefährdungspotenzial durch Leckagen o.ä. beim Hydraulikeinsatz verhindern sollen.

\subsection{Mobileinheit}

Der GMH05 wird gleichsam der Tritucap in einer Schlepper- Drei- PunktHydraulik in Rückwärtsfahrt des Schleppers betrieben. Der Hauptantrieb der wesentlichen Aggregate erfolgt über die Zapfwelle des Schleppers.

Die Bergung des Häckselmaterials auf der Fläche erfolgt auch hier durch den Frontladercontainer. Das Häckselgut wird auch hier über den Schlepper hinweg in den Container verladen.

\subsubsection{Praxiseinsätze des GMH 05}

Die Praxiseinsätze erfolgten nach Fertigstellung des GMH 05 wieder auf den Versuchsflächen. Die eingesetzte Mobileinheit aus Schlepper, Anbaumähhäcksler und Frontladercontainer ist in Abb. 44 zu sehen.



Abb. 44: Ansicht des Gesamtmaschinensystems zur vollmechanisierten Entbuschung mit dem Gehölzmähhäcksler 05 


\subsection{Schneiden}

Das Schneiden einzelner Triebe ist mit dem Sägeblattbalken bis zu einem Durchmesser von $12 \mathrm{~cm}$ möglich. Bei darüber hinausgehenden Gehölzdurchmessern stößt der sich unter den Sägeblättern befindliche Getriebekasten des Stirnradgetriebes gegen den Stumpf und hebt die Maschine leicht aus. Dieses führt während des Schnittvorgangs zu starken vertikalen Kräften, die derart auf die Sägeblätter wirken, dass sich diese durch Verwindungen an den überlappenden Stellen berühren und beschädigen können. Unterhalb dieses Durchmesserbereiches kann kein einschränkender Einfluss auf den Schneidprozess durch den Getriebekasten festgestellt werden.

In Abb. 45 ist beispielsweise ein mit dem Sägeblattbalken durchtrennter Weidenstumpf zu sehen. Der Durchmesser der Schnittfläche beträgt $12 \mathrm{~cm}$. Der Versuch den Stumpf im bodennahen Bereich mit $15 \mathrm{~cm}$ Durchmesser zu trennen, scheitert durch den oben beschriebenen Zusammenhang.

Die auf den äußeren Sägeblätter aufgesetzten Förderzylinder erfüllen ihre angedachte Aufgabe ohne nennenswerte Mängel. An den Außenseiten geschnittenes Gehölzmaterial wird zu den Zuführaggregaten befördert.

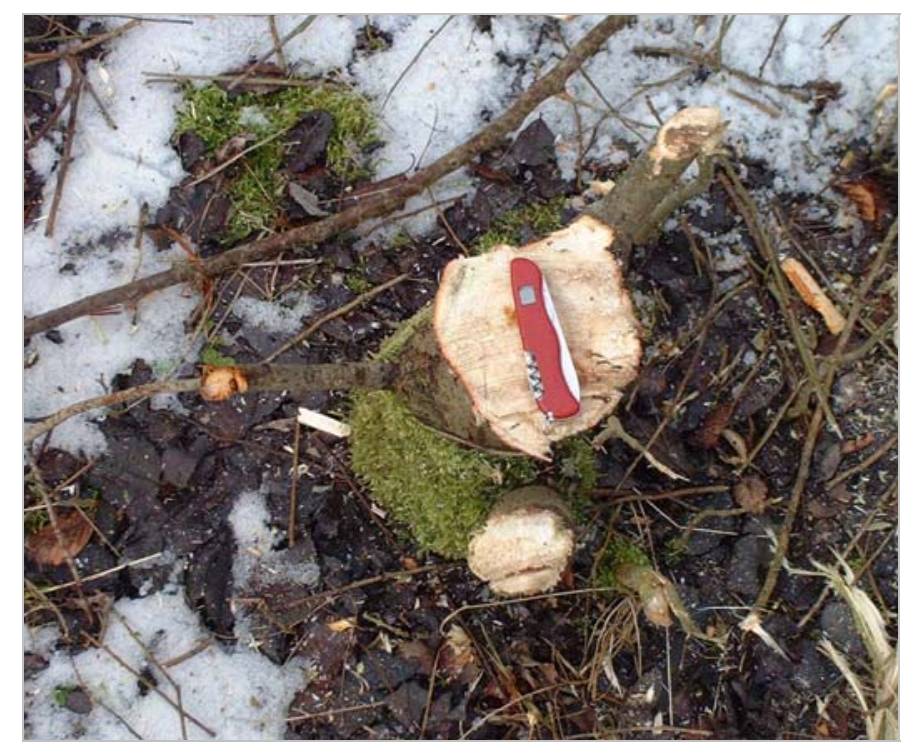

Abb. 45: Schnittfläche eines mit dem Sägeblattbalken des Gehölzmähhäckslers 05 durchtrennten Weidentriebs 
Das Vorspannen der Triebe mit dem Druckbügel in Fahrtrichtung lässt den erwarteten Schleudereffekt zu den Einzugsorganen erkennen und die Triebe lassen sich während des Schneidens in eine annähernd waagerechte Einzugsposition ausrichten. Prinzipiell lässt sich somit der Effekt aus der Kurzumtriebsbewirtschaftung auch auf die Gehölze der Verbuschungen übertragen.

In zunehmend dichter werdender Vegetation und größeren WHD wird es jedoch schwieriger die Gehölze zwischen den in Fahrtrichtung stehenden Bestand zu drücken. Einerseits nimmt der Gegendruck mit steigendem Durchmesser und höherer Triebdichte zu und andererseits wird der benötigte Raum zwischen den in Fahrtrichtung stehenden Gehölzen durch größer werdende und dichter stehenden Gehölzkronen geringer. Die Folge ist, dass die abgetrennten Stümpfe durch einen zu starken Gegendruck an deren Kronenbereich wieder aus dem Einzug gehebelt werden oder eine waagerechte Ausrichtung nicht möglich ist. Besonders ausgeprägt ist dieses in sehr dichten buschartigen Gehölzen der Fall- vor allem wenn diese auch noch durch Dornen verhakt und ineinander verwachsen sind. Es scheint entsprechend eine Grenze der Bearbeitbarkeit nach diesem Arbeitsprinzip zu bestehen. In Abb. 46 ist beispielsweise eine sehr dichte Verbuschung mit vermehrt dickeren Weißdornbüschen auf der Fläche DB 3 zu sehen. Die waagerechte Ausrichtung der Gehölze ist hier nicht mehr möglich, weshalb die Bearbeitung dort abgebrochen werden muss. 


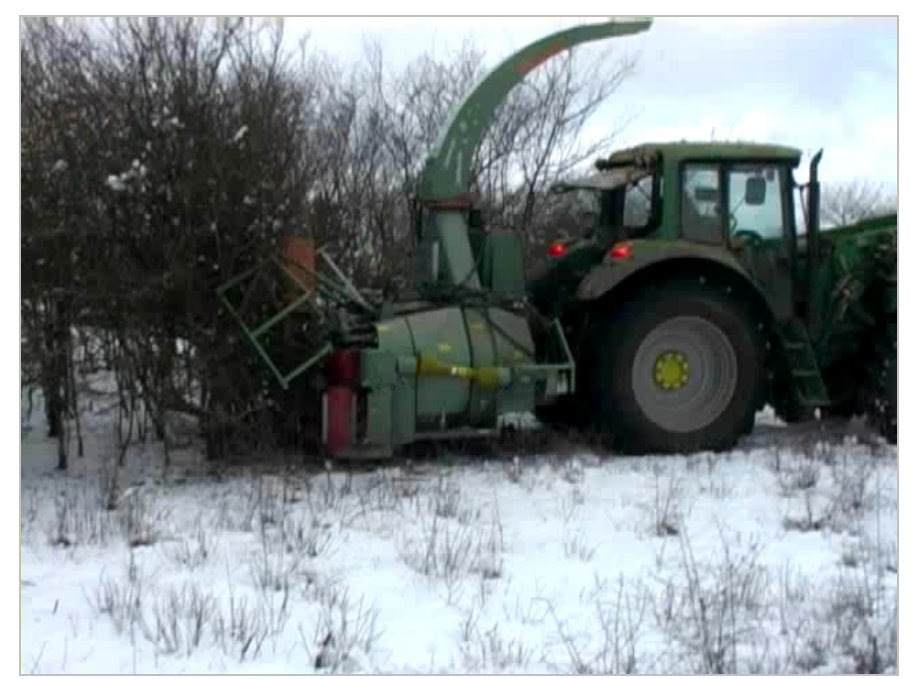

Abb. 46: Hohe Bestandesdichte und vermehrt dickere Weißdornbüsche behindern waagerechte Ausrichtung der Gehölze mit dem Gehölzmähhäcksler 05

In baumartigen Gehölzen ist das Arbeiten aufgrund der geringeren Kronendurchmesser auch bis in sehr dichte Bestände eher möglich. Die Bearbeitung wird jedoch durch die Biegesteifigkeit des Bestandes, welche durch die Vielzahl an Trieben stark zunimmt, erschwert (Abb. 47). Fasst man dieses zusammen, dann wird das Entbuschungsvermögen des Schneidens und Vorspannens nach diesem Arbeitsverfahren nicht nur durch den WHD sondern auch durch die Biegesteifigkeit ${ }^{3}$, die Wuchsform und die Bestandesdichte begrenzt.

Die Bügelposition muss deshalb auch je nach Bestandesbedingungen angepasst werden. Dünne Triebe sind allgemein biegsamer, so dass sie stärker vorgebogen werden müssen, um eine ausreichende Spannung des Triebes vor dem Schnitt zu erreichen. Bei zunehmender Höhe der Gehölze muss der Bügel des Weiteren auch höher angesetzt werden, da die Triebe sonst über den Bügel hinweg auf den Schlepper zu kippen drohen.

\footnotetext{
3 Die Biegesteifigkeit ist vereinfacht gesehen ein Maß für das Verhältnis von Biegemoment und Krümmung und beinhaltet letztlich das Flächenträgheitsmoment (Bezug zum Durchmesser) und Elastizitätsmodul (Bezug zum Material) (GROTE ET AL., 2007).
} 


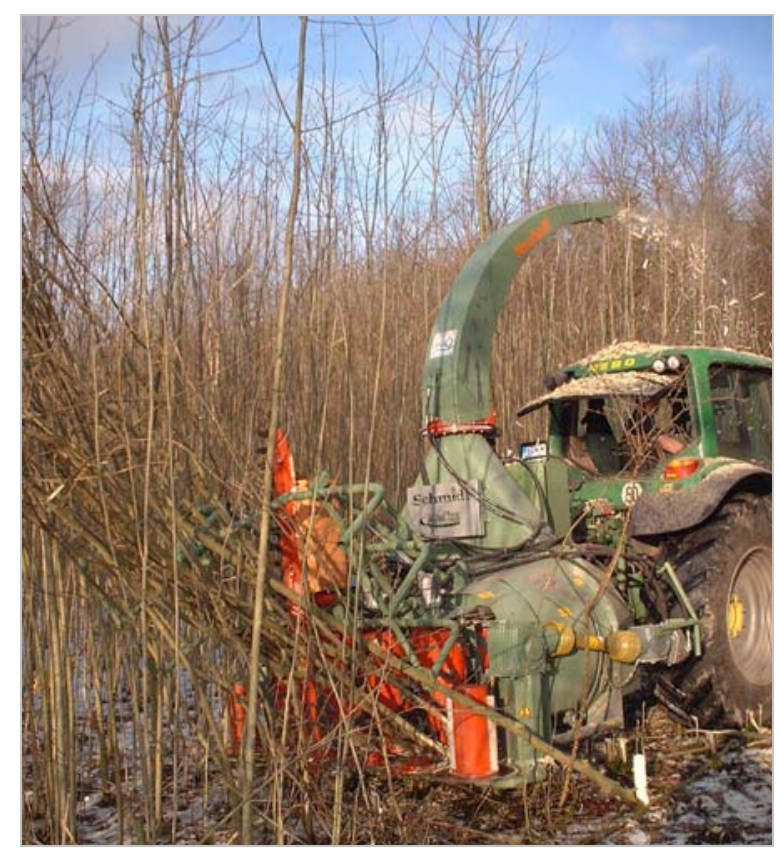

Abb. 47: Gehölzmähhäcksler 05 bei der Mähernte im dicht mit Eschen verbuschten Bereich auf der Ausgleichsfläche in Roringen

Vereinzelt kommt es vor, dass Triebe auch diagonal oder gar quer zur Arbeitsrichtung fallen. Diese stören anschließend den Arbeitsprozess, da sie nicht mehr aufgenommen werden können und die weitere Zuführung unterbinden. Vor allem vor dem Einsatz der hydraulischen Astschere bleibt nur die händische Beseitigung oder das Hinwegfahren über das Gehölzmaterial (Abb. 48). Bei querfallendem Holz ist letztlich der Anspruch, den dieses Arbeitsprinzip an die Ausrichtung der Trieb setzt, nicht mehr erfüllt. 


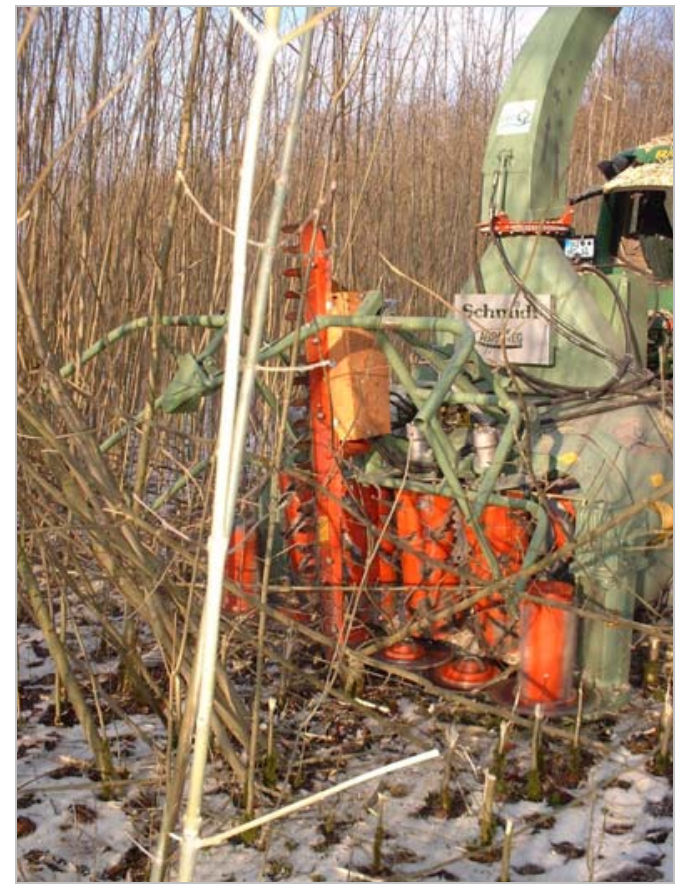

Abb. 48: Diagonal bzw. quer zur Zuführrichtung liegende

Gehölztriebe als Störung vor den Aufnahmeaggregaten des

Gehölzmähhäckslers 05

\subsection{Aufnehmen und Zuführen}

Die Zuführtrommeln müssen im Rahmen der Einsätze mehrfach bezüglich der Förderwerkzeuge überarbeitet werden. Die anfänglich montierten Schneckenwendeln zeigen eine zu geringe tangentiale Förderwirkung in Richtung der Zerkleinerungsaggregate auf, so dass es zu Materialstauungen zwischen innen kommt. Des Weiteren werden einzelne waagerechte Triebe durch die axiale Förderwirkung der Schneckenwendeln so tief in den Sägeblattbereich transportiert, dass diese bei der Zufuhr zum Häckselaggregat längs zur Triebachse eingeschnitten werden (Abb. 49). Die Blockade des Sägeblattantriebs ist die Folge. Die Flachstähle im unteren Trommelbereich neigen dagegen zum Wickeln von dünnen Ästen, so dass es zu Blockaden kommt, da sich diese zwischen Flachstahl und Gehäuse einklemmen. Eine Verkürzung der Werkzeuge auf dem Walzenkörper verringert zwar diese Wickelhäufigkeit, reduziert jedoch deren Förderleistung stark, was sich wiederum in Materialstauungen äußert. 


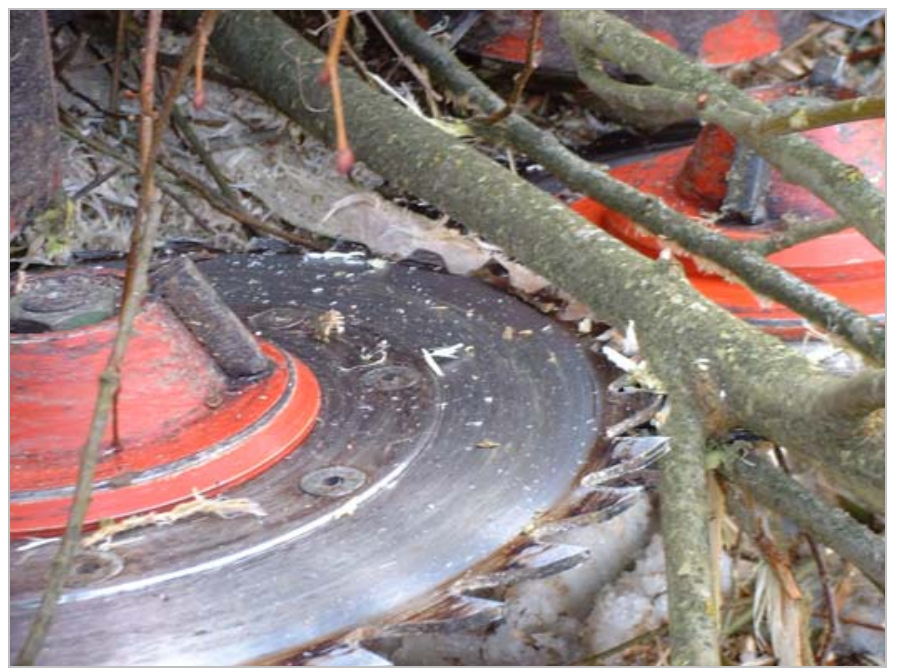

Abb. 49: Durch zu tiefe Förderung der Zuführwalzen

hervorgerufene Auftrennung von Trieben längs zur Triebachse

Versuche die tangentiale Förderwirkung der Schneckenwendeln durch Zahnung verhältnismäßig zu erhöhen (ohne Abb.), scheitert an deren erhöhter Wickelneigung, die besonders in den feiner verzweigten Büschen ausgeprägt ist. Es ist aufgrund der heterogenen Bestandesverhältnisse nur sehr schwer möglich, den richtigen Grad zwischen Förderwirkung und Wickelneigung zu ermitteln.

Dabei hat auch die Wuchsstruktur einen erheblichen Einfluss. Buschartige Gehölze haben einen sparrigen und voluminösen Wuchs, weshalb sie beim Durchführen zwischen zwei Zylindern bzw. Walzen einen größeren Widerstand aufbringen, gleichzeitig besitzen sie eine Vielzahl feinerer und stark von der Triebachse abstehender Äste, die die Wickelneigung an den Förderelementen erhöhen (siehe auch FIRUS \& BELTER, 1995B; WIENEKE, 1957). Eine Vergrößerung des Walzendurchmessers, wie WIENEKE (1957), sie vorschlägt, ist baulich nicht möglich. Letztlich können mit den gezahnten und $45^{\circ}$ geneigten Fördersegmenten Werkzeuge ermittelt werden, deren Wickelneigung bei den Einsätzen sehr gering ist und die dennoch eine ausreichende Förderwirkung erreichen (Abb. 50). Das Auftreten von Blockaden und Materialstauungen kann so drastisch reduziert werden. 


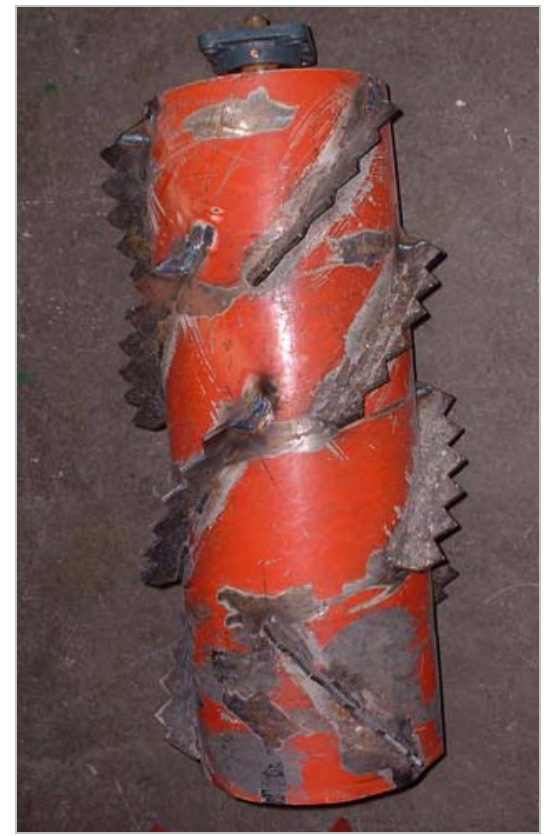

Abb. 50: Mit gezahnten Förderelementen ausgestatteter Förderzylinder des Gehölzmähhäckslers 05

Da der Antrieb der Zuführtrommeln über die Hydrauliksteuergeräte vom Schlepper aus erfolgt, kann der Bediener nun auch in die Materialzuführung aktiv eingreifen. Dieses erweist sich als sehr nützliches Werkzeug, um eine der Situation angepasste Materialmenge zuführen zu können. Teilweise können durch die Unterbrechung des Antriebs der Zuführeinrichtungen auch Störungen, wie verklemmtes Material, beseitigt werden, so dass dieses den Arbeitsablauf erheblich erleichtert. Dieses Bedienungs- und Zuführwerkzeug stellt eine erhebliche Verbesserung zur Tritucap dar.

\subsection{Zerkleinern}

Die Zerkleinerung mit den Schneckenhäckslerrotoren erweist sich prinzipiell als vorteilhaft. Der Selbsteinzug der Häckselaggregate unterstützt durchaus den Einzugsprozess, indem weitere Gehölze von den gerade bearbeiteten mitgezogen werden. Bei einer drohenden Überlastung durch zu viel Material kann der Bediener diesem in der Regel durch das Abstellen der Zuführwalzen begegnen. Der Selbsteinzug offenbart bei einer Überlastung jedoch auch eine nachteilige Seite des Systems. Wird das Häckselaggregat durch eine zu hohe Materialzufuhr und nicht ausreichender Antriebsleistung zum Stillstand gebracht, so können die im Häckselspalt verbliebenen Reste 
der Triebe nicht einfach durch Herausziehen oder eine Drehrichtungsumkehr der Zuführaggregate entfernt werden. Die Schneiden stecken in einer Art formschlüssigen Verbindung in den Trieben. Dabei sind die Triebe durch einen hohen radialen Druck vom Häckselrotor zwischen dessen Schneiden und dem Zuführboden verklemmt. Der Rotor muss manuell und mit hohem Kraftaufwand mit einem Hebelwerkzeug entgegengesetzt zur eigentlichen Drehrichtung gelöst werden, indem die Triebe praktisch wieder ein Stück „herausgeschraubt“ werden. Erst nachdem die Schneiden sich durch den verringernden Konusumfang aus dem Holz zurückziehen, können die Triebe entnommen werden. Je nach Intensität dieser Rotorblockaden kann deren manuelle Beseitigung sehr zeit- und energieaufwendig sein. Dieses stört den Arbeitsprozess erheblich und führt durch die manuellen Arbeiten im Bereich der Zerkleinerungswerkzeuge zu einer Gefährdung der Bediener.

\subsubsection{4. Überladen}

Hinsichtlich des Überladens kann die Materialzusammenführung beim Übergang zum Überladekrümmer als Schwachstelle identifiziert werden. In diesem Bereich treten vermehrt Störungen durch Verstopfungen auf. Dort prallt das zerkleinerte Material in einem Winkel von ca. ${ }^{\circ} 70^{\circ}$ von den zwei Beschleunigern aufeinander und muss dann jeweils um $35^{\circ}$ in den Überladekrümmer umgelenkt werden. Eine unzureichende Beschleunigung kann bei der vorliegenden Umfangsgeschwindigkeit der Auswerfer nicht der Grund für dortige Materialstauungen sein. Dieses entspricht der Beobachtung, dass ein großer Teil der Hackstücke mit hoher Geschwindigkeit übergeladen wird, während ein kleinerer Teil, in Hackstückform und -größe vergleichbar, den Überladecontainer mangels Wurfweite nicht erreicht. Ein Vergleich zu im Überladecontainer aufgefangenem zu nicht aufgefangenem Material ergibt Mengenanteile von bis zu 20\% am Gesamthäckselgut, die den Container nicht erreichen. Die Aufteilung des Gutstromes in der Maschine stellt somit auch einen höheren Anspruch diesen wieder zu vereinigen und führt offensichtlich zu einem zu hohen Energieverlust des Hackgutes aufgrund einer suboptimalen Gutführung. Offensichtlich besteht hier eine ungünstige Auswurfgeometrie. 
Im Bezug auf den Materialtransport auf der Fläche und der Bergung des Hackmaterials kann sich der Überladecontainer, wie es sich schon bei den Einsätzen der Tritucap abzeichnet, auch beim GMH 05 bewähren. Aufgrund des größeren mit dem GMH 05 bearbeiteten Flächenumfangs werden vermehrt Überladevorgänge des geborgenen Materials auf Transportfahrzeuge durchgeführt. Bei den Versuchseinsätzen wird dazu ein landwirtschaftlicher Anhänger genutzt. Das Überladen zeichnet sich durch das einfache Auskippen des Containers als sehr zeiteffizient aus. Der Überladevorgang kann innerhalb einiger Minuten erfolgen und eine dosierte Beladung ist durch die hydraulische Werkzeugbetätigung des Frontladers gegeben.

\subsection{Antriebskonzept}

Hinsichtlich des komplexen Antriebskonzeptes treten mehrere schwerwiegende Schäden durch Drehmomentüberlastungen bei der Leistungsübertragung zu den einzelnen Maschinenkomponenten auf. Diese resultieren weniger aus einem zu hohen Leistungsbedarf bzw. einer zu hohen Übertragung vom Schlepper aus. Sie müssen von schädlichen Drehmomentspitzen zwischen einzelnen Komponenten am GMH 05 ausgehen, da sie auch an unbelasteten Komponenten auftreten. So bricht z.B. die Antriebswelle zum unbelasteten Sägeblattantrieb während ein Häckselrotor eine hohe Belastung erfährt.

Der Hauptantrieb des GMH 05 erfolgt fast ausschließlich über die Gelenkwelle vom Schlepper aus und verzweigt sich dann stark. Durch die mechanische Verbindung der Komponenten untereinander kann eine belastete von einer unbelasteten Komponente zusätzlich zur übertragenen Schlepperleistung eine Leistung aus deren Trägheitsmoment übertragen bekommen. Dieses führt neben einer erhöhten Gesamtleistung in einem Antriebsstrang zudem zu ständigen Lastwechseln, die mit starken Drehmomentspitzen einhergehen können. BLOCK (2004) berichtet von gleichen Problemen bei der Entwicklung der Tritucap. In den Entbuschungseinsätzen erweist sich das Antriebssystem der Tritucap jedoch als sehr robust, weshalb es auch beim GMH 05 eingesetzt wird. 
Anscheinend führt hier die zusätzliche Schwungmasse des Sägeblattbalkens zu einem stark unterschätzten Einfluss. Aufgrund der zusätzlichen Komponenten sind die Lastwechsel im Fall des GMH 05 so stark ausgeprägt, dass Getriebe innerhalb kurzer Zeit und teils mehrfach durch gebrochene Zahnflanken oder gar gebrochene Wellen zerstört werden.

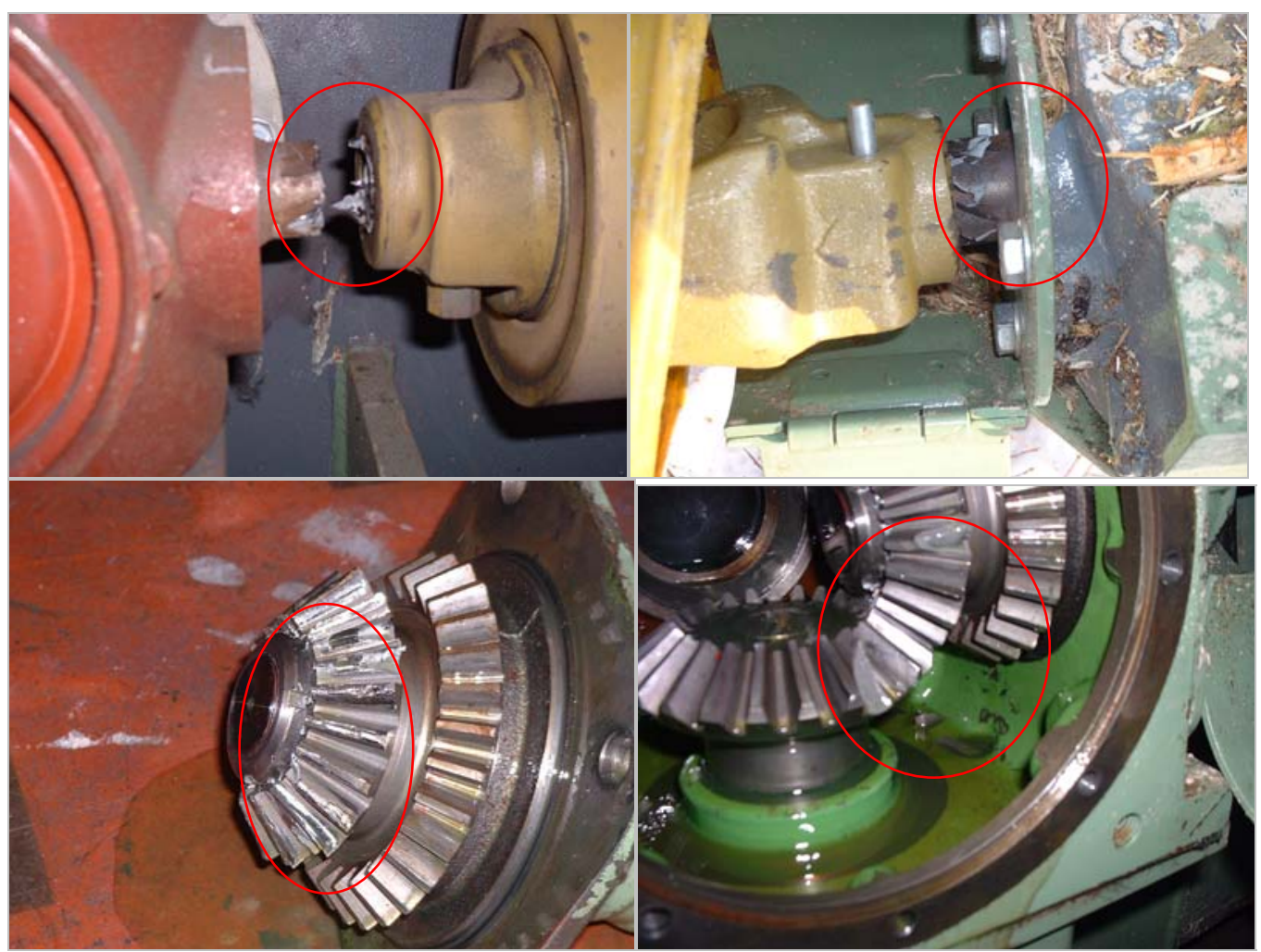

Abb. 51: Schäden im Antriebsstrang des Gehölzmähhäckslers 05 durch schädliche Lastwechsel und Lastspitzen

Von einem Versuch die auftretenden Drehmomente mit einer Drehmomentmessnabe zu ermitteln, wird aufgrund zu erwartender Schäden an der Messnabe durch die offensichtlich hohen Werte abgesehen. Der Versuch zusätzliche Rutschkupplungen und Freiläufe zu integrieren, scheitert entweder aufgrund des zu geringen Bauraumes oder aufgrund einer zu geringen Wirkung, da das weitere Auftreten von Schäden auch dann nicht wirksam vermieden werden kann. Dieses Problem kann im weiteren Verlauf technisch nicht behoben werden und stellt eine große Schwachstelle des Maschinenkonzeptes dar. 


\subsection{Mobileinheit}

Bei den Ersteinsätzen zeigen sich spürbar die Nachteile der gestiegenen Abmaße und Gewichte des GMH 05 im Gegensatz zur Tritucap. Die großflächige Basis des Auswurfturms zur Materialzusammenführung der beiden Häckselrotoren sowie auch die Zuführaggregate direkt vor dem Schneidapparat lassen keinen Blick auf den Schneidbereich zu. Der Bediener kann durch die Maschinenlänge nur in einem flachen Blickwinkel über die Maschinenaufbauten schauen, weshalb selbst der angefahrene Arbeitsbereich vor den Sägeblättern auf einer Länge von bis zu 1,5 m nicht einsehbar ist. Aus diesem Grund wird ein Weitwinkel- Kamerasystem über dem Arbeitsbereich installiert, wie sie im Landwirtschaftsbereich in unübersichtlichen Maschinenbereichen (z.B. Rübenroder, Mähdrescher) verwendet werden. Über einen Monitor auf dem Schlepper kann der Bediener so in den Arbeitsbereich, in den Schneidbereich sowie auch zu gewissen Teilen in den Häckselbereich blicken, was die Beurteilbarkeit des Arbeitsprozesses ausreichend ermöglicht. Ein Kamerasystem erscheint auch als die einzige Möglichkeit, diese Bereiche bei dem Maschinenkonzept der Mähernte einsehen zu können. Auch beim Einsatz der Tritucap wäre dieses zur Verbesserung der Sicht in den Arbeits-/ Häckselbereich sehr hilfreich.

Die Gesamtlänge der Maschine stellt zudem höhere Anforderungen an den Bediener hinsichtlich der Höhenführung. Durch den langen Hebel wirken sich Bodenunebenheiten im Vergleich zur Tritucap weit stärker auf die eingestellte Schnitthöhe aus. Da sich der Achsabstand am Basisfahrzeug nicht geändert, aber der Abstand der Schneiden zur Hinterachse des Schleppers als Drehpunkt fast verdoppelt hat, muss nach dem Hebelgesetz von einer annähernd doppelt so starken Beeinflussung ausgegangen werden. Korrektureinstellungen der Schnitthöhe müssen weit häufiger ausgeführt werden, um einen bodennahen Schnitt gewährleisten zu können.

Auch hinsichtlich der Manövrierfähigkeit wirkt sich die Länge des GMH 05 negativ aus. Es wird zum Rangieren weit mehr Platz benötigt und bei Richtungskorrekturen während der Entbuschungsarbeiten schwenkt die Maschine stärker seitlich aus. Durch seitlich stehende Bäume ist dieser 
Raum teilweise nicht gegeben, so dass die Richtungskorrekturen dann durch einen zwischenzeitlichen Richtungswechsel vollzogen werden müssen. Der relativ weit vom Schlepper entfernte Schwerpunkt der Maschine und deren relativ hohes Gewicht erschweren das Manövrieren zudem, da seitliche Schwingungen in der Drei-Punkt- Hydraulik zunehmen und das Gesamtfahrzeug auf Lenkbewegungen schwerfälliger reagiert. Besonders bei zunehmender Schräge auf der Fläche und beim Überfahren der Stümpfe wirkt sich dieser Effekt stark aus.

\subsection{Hackschnitzelqualität}

In Abb. 52 sind die Ergebnisse der Untersuchungen zu den produzierten Hackschnitzeln aufgezeigt. Sie stammen aus einer Mischprobe von ca. 9 Kubikmeter Hackschnitzel, die zu ca.50\% aus baum- sowie buschartigem Material besteht. Bei den Entbuschungsmaßnahmen wurden alle bearbeitbaren WHD von $<1 \mathrm{~cm}$ bis $12 \mathrm{~cm}$ bearbeitet. Der Wassergehalt der Probe beträgt 26 \% nach sechsmonatiger Lagerung.

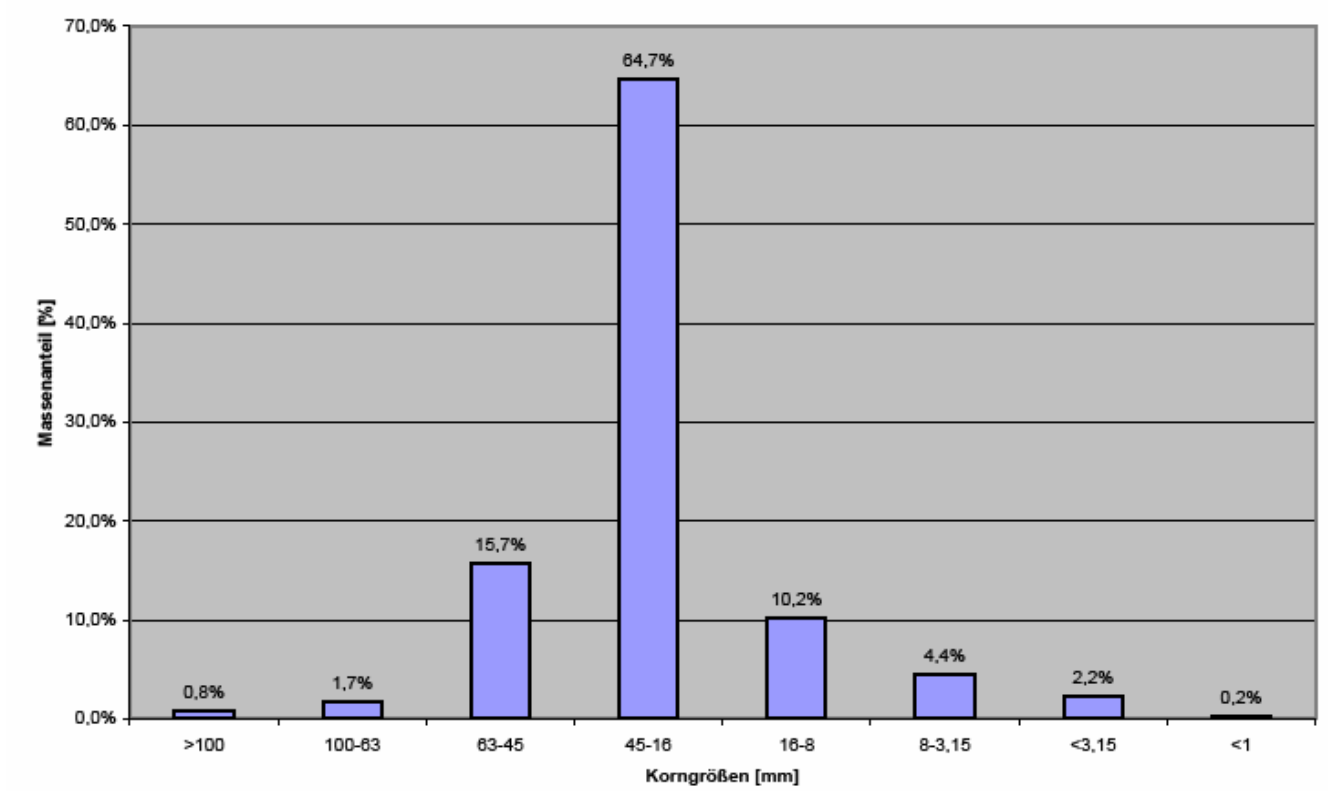

Abb. 52: Ergebnisse der Siebanalyse nach CEN/TS 15149-1 einer Hackschnitzelmischprobe produziert mit dem Gehölzmähhäcksler 05

Die Hauptfraktion der mit dem GMH 05 produzierten Hackschnitzel liegt in der Größe 45-16 mm. In diesem Größenbereich kann der mit Abstand 
größter Massenanteil mit 64,7\% bestimmt werden. Entsprechend der Ergebnisse in Abb. 52 lässt sich das Hackgut nach CEN/TS 14961 (2005) als Holzhackschnitzel in der Handelsform P63 einstufen (Tab. 5).

Tab. 5: Einordnung der Siebanalyse einer Mischprobe des Häckselmaterials des Gehölzmähhäckslers 05 nach CEN/ TS 15149; Kastenlänge unter Größenbereichen entspricht deren Summe; gestrichelter Kasten entspricht der Einordnung; n.a. = nicht aufgenommen

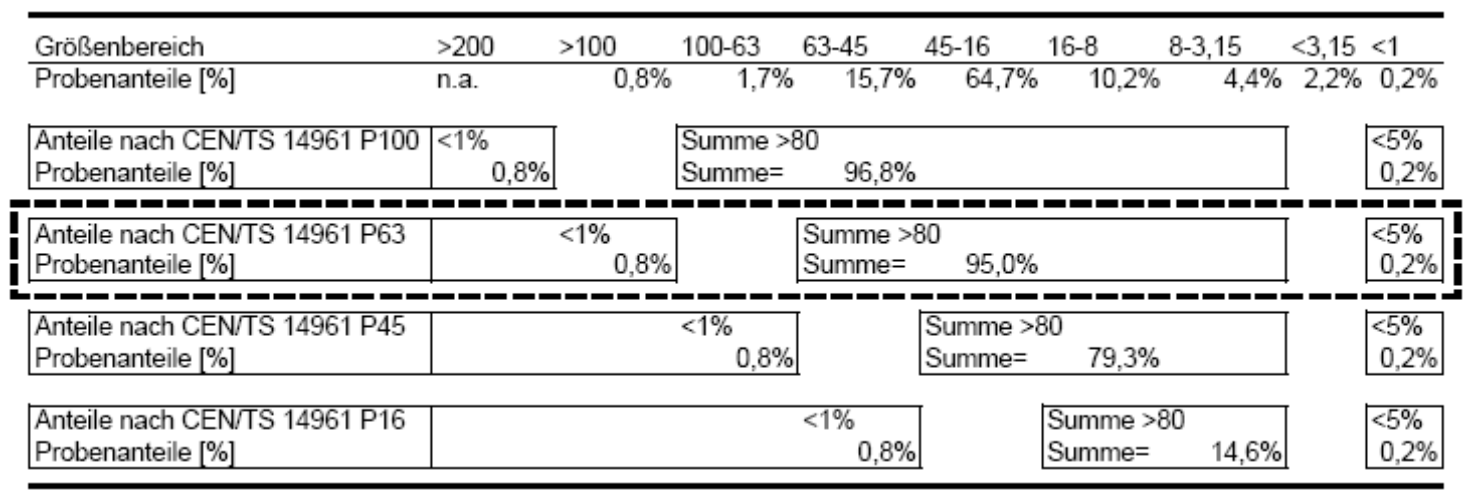

Die in den Versuchen produzierten Hackschnitzel aus der vollmechanisierten Entbuschung von Offenflächen sind somit nach gängiger Norm von ihrer Aufarbeitungsqualität her als marktfähig einzustufen. Das heißt, dass mit dem GMH05 ein nach der äußeren Beschaffenheit verwertbares Hackschnitzelprodukt produziert werden kann. Einschränkend muss dazu gesagt werden, dass die Brennstoffnorm CEN/TS 14961 (2005) auch Grenzwerte wie z.B. den Aschegehalt und den Stickstoffgehalt beinhaltet. Deren Anteile wurden hier nicht bestimmt. Sie können z.B. bei einer verstärkten Aufnahme von Gras oder Erde sicherlich Relevanz erlangen. BAUER (2005) führt dazu aus, dass bei Landschaftspflegehackschnitzel prinzipiell mit einem erhöhten Anteil von Störstoffen zu rechnen sei, die Hackschnitzelgrößenverteilung und der Wassergehalt aber wichtigstes Kriterium für eine Verwertbarkeit darstellen würden. Entsprechend erfüllt der GMH 05 eines der wichtigsten Kriterien zur Produktion eines verwertbaren Landschaftspflegeproduktes. 


\subsubsection{Evaluation des GMH 05}

Der GMH 05 stellt hinsichtlich der Einsatzfähigkeit in der Entbuschung von Offenflächen eine Verbesserung im Vergleich der Tritucap dar. Durch die Trennung von Schneid- und Zerkleinerungsbereich sowie die dadurch entstandene Möglichkeit zum Einsatz geeigneter Zuführaggregate können vollmechanisierte Entbuschungen auf Offenflächen durchgeführt und eine grundlegende Funktion des schlepperbasierten Konzeptes zur Mähernte von sukzessiv gewachsener Gehölzvegetation bestätigt werden. Buschartige sowie auch baumartige Sukzessionsgehölze können bis zu einem Durchmesser von $12 \mathrm{~cm}$ vollmechanisiert geschnitten, aufgenommen und zerkleinert werden. Dabei kann das Material, wenn auch mit Verlusten, geborgen werden. Der GMH 05 kann somit grundlegende Anforderung an das Verfahrenskonzept erfüllen. Eine Vergrößerung des Entbuschungsvermögens ist dabei sicherlich wünschenswert. Der technische Stand hinsichtlich der Bearbeitbarkeit der Gehölze erfüllt aber bis dahin die Anforderungen des angestrebten Einsatzbereiches.

Der durch die Komponententrennung entstandene hohe Verzweigungsgrad des mechanischen Antriebsstranges begrenzt jedoch die grundsätzliche Einsatzfähigkeit des Maschinenkonzeptes. Die unbeherrschbaren und schädlichen Belastungen auf die Komponenten lassen keinen zuverlässigen und praxisgerechten Betrieb zu. Im Bezug auf die Haltbarkeit und der Abstimmung der einzelnen Komponenten untereinander kann das derartig gestaltete Maschinenkonzept des GMH 05 somit nicht den Zielvorstellungen entsprechen.

Hinsichtlich der Übersichtlichkeit kann der GMH 05 nach einigen Verbesserungen die Ansprüche ausreichend zufrieden stellen. Das eingesetzte Kamerasystem ermöglicht auch den Blick in den Häckselbereich, weshalb nicht nur die Zuführkomponenten, sondern auch der Zustand der Häckselaggregate visuell beurteilt werden können. Dieses stellt eine erhebliche Verbesserung zur Situationsbeurteilung dar.

Durch die Steuerbarkeit der Zuführkomponenten ist bei diesem Konzept auch eine Möglichkeit zur Einflussnahme durch den Bediener auf den 
Zuführprozess gegeben. Eine situationsangepasste Materialmenge kann somit zu den Zerkleinerungsaggregaten befördert werden, wodurch sich Überlastungen durch zu große Materialmengen wirksam vermeiden lassen. Die Bedienung der Höhenführung zur Erreichung eine bodennahen Schnitthöhe ist bei dem Konzept nur bedingt gegeben. Überlegungen den Bediener durch zusätzliche Stütz- oder Tasträder zu unterstützen, müssen verworfen werden, da sich allein durch die verbleibenden Stümpfe unter der Maschine Fehlmessungen mit starken Höhenschwankungen einstellen würden. Gleichsam unbefriedigende Ergebnisse sind auch bei laser- oder ultraschallbasierten Systemen zur Höhenüberwachung zu erwarten, da diese neben der Bodenoberfläche auch die höhergelegenen Schnittflächen der Stümpfe mit aufnehmen würden. Da sich eine vorerst ausreichende Höhenführung, wenn auch eingeschränkt, realisieren und im Weiteren auch durch z.B. eine Reduzierung der Maschinenlänge verbessern lässt, wird in diesem Stadium der Entwicklung von einer intensiveren technischen Lösungsfindung dieses Themas abgesehen. Sie kann in einem späteren Entwicklungsstadium durchaus wieder aufgenommen werden.

Bezüglich der Manövrierbarkeit und der allgemeinen Übersichtlichkeit ist das Maschinenkonzept nur unbefriedigend gelöst. Die Aufbauten sind zu stark im notwendigen Sichtbereich positioniert. Der Auswurfkrümmer muss seitlich angeordnet sein, um dem Bediener ein besseres Sichtfeld zu verschaffen. Zudem muss er eine Überarbeitung hinsichtlich der Überladeleistung bzw. der Gewährleistung einer annähernd vollständigen Bergung erfahren. Der Materialanteil, welcher nicht geborgen wird, ist zu hoch.

Das Maschinengewicht und die Länge des Maschinenkonzeptes sind als zu groß einzustufen, weil sie das Arbeiten mit dem Gerät grundsätzlich erschweren. Die größere Hebelwirkung, welche durch Länge und Gewicht auf das Basisfahrzeug wirkt, sorgt zusätzlich für eine stärkere Belastung der Hinterachse, weshalb dort mit einer erhöhten Einwirkung auf den Boden zu rechnen ist. Anhand von Penetrometer- Messungen zum Einfluss des Einsatzes auf das Bodengefüge können KESTING \& ISSELSTEIN (2006) zwischen Einsatz- und Kontrollflächen keine signifikanten Unterschiede 
feststellen. Die Einsätze sind jedoch auch durch günstige Witterungs- und Bodenbedingungen beeinflusst und können so nur als bedingt aussagekräftig zum Einflusspotenzial der Maschinentechnik gewertet werden. Prinzipiell sollten zur Verbesserung der Fahr- und Bedienungseigenschaften sowie auch zur Verminderung eines Gefährdungspotentials für den Boden das Maschinengewicht und die Länge der Maschine in der weiteren Konstruktion vermindert werden. Dieses erscheint zu diesem Zeitpunkt der Entwicklung auch grundlegend möglich, da die Faktoren Länge und Gewicht bisher hinter der Funktionsfähigkeit zurückstehen. Die Verwendung leichterer Materialien bzw. das Potenzial zur Einsparung von Konstruktionsmaterial kann ausreichend ermittelt werden. Allein durch eine Optimierung der Anbaukonstruktion der Maschine erscheint alleine eine Reduktion der Länge von bis zu $30 \mathrm{~cm}$ möglich (SCHMIDT, 2006).

Die Aufteilung des Gutstromes in zwei voneinander getrennte Ströme erweist sich in diesem Maschinenkonzept als sehr ungünstig. Einerseits trägt die quasi doppelte Ausführung der Komponenten zu dem Entstehen des hohen Eigengewichtes bei und die zusätzlich notwendige Gutstromteilung führt zu einer möglichen Problemstelle. Deren Störpotenzial im Arbeitsprozess ist zwar durch die Umbauten gemindert, die Lösung geht jedoch mit dem zusätzlichem Gewicht der Astschere und dem Entstehen von den Aufnahmeprozess behindernden Kurzholzstücken einher. Die Aufteilung des Gutstroms mit ihren Folgen kann in dem Konzept nicht beibehalten werden, da deren Nachteile letztlich zu groß sind.

Der Einsatz des Schneckenhäckslersystems kann durchaus positiv bewertet werden. Der Selbsteinzug des Systems unterstützt merklich den Aufnahmevorgang. Die Beseitigung von auftretenden Blockaden bedarf bei seiner weiteren Verwendung jedoch einer technischen Lösung. Trommelund Scheibenradhackersysteme mit Materialzuführungen erreichen eine wirkungsvolle Vermeidung von Überlastungen durch einfaches Abstellen der Zuführaggregate. Dabei soll je nach Häckslerauslegung ein Selbsteinzugseffekt zwar nicht immer zu verhindern sein, jedoch können auftretende Blockaden relativ unkompliziert durch ein leistungsarmes 
Reversieren der Zuführaggregate gelöst werden (BRUSCHE, 1983). Dieses ist beim Schneckenhacker so nicht ausreichend möglich. Bei Standardschneckenhackern zur manuellen oder zur Kranbeschickung werden z.B. mechanisch bzw. hydraulisch schwenkbare Zuführböden eingesetzt, um den Abstand zwischen Schneide und Zuführboden vergrößern zu können (System der Fa. Laimet, Finnland). So kann die Verbindung zwischen Schneide und Holz gelöst und das Holz per Hand oder Kran wieder entnommen werden. Bei einer Kombination des schwenkbaren Zuführbodens mit einer Reversierung der Zuführaggregate bestünde die Möglichkeit Blockaden des Schneckenhäckslerrotors bei dem hier angewendeten vollmechanisierten Mäherntesystem vom Bedienerplatz und ohne manuelle Hilfe zu beseitigen.

Das Bergungskonzept mit dem eingesetzten Frontladercontainer lässt sich bestens in das vollmechanisierte Entbuschungskonzept integrieren. Einerseits sind neben den Standardkomponenten Frontlader und Schaufel nur geringe Umbauten notwendig und andererseits erfüllt es alle Anforderungen hinsichtlich Funktion und Betriebssicherheit. Der Transport des Häckselgutes auf der Fläche und die Bergung lassen sich beanstandungsfrei realisieren. Die Abstimmung der Bergungskapazität des Frontladercontainersystems wird sicherlich bei einem abschließend entwickelten Maschinenkonzept einer Abstimmung bedürfen, um den Gesamtentladeaufwand möglichst gering zu halten. Dabei werden jedoch auch Aspekte wie Gewichtsbelastung, Fahrstabilität und Übersichtlichkeit beachtet werden müssen.

Das mit dem GMH 05 produzierte Häckselgut kann in eine gültige Hackschnitzelnorm einklassiert werden. Die dazu verwendete Mischprobe ist keiner bestimmten Vegetationsstruktur zugeordnet und stammt aus Einsätzen über den gesamten Anwendungsbereich hinweg. Es kann somit nur bedingt eine Aussage über die Zusammensetzung des bearbeiteten Materials gemacht werden. Hohe Anteile an schlecht zu bewertendem Ausgangsmaterialien, wie Dünnreisig oder weniger verholztem Material oder gar Blättern und Gras, haben einen entscheidenden Einfluss auf die 
resultierende Qualität und die Verwertungsmöglichkeiten des Hackschnitzelproduktes bei der Zerkleinerung (BAUER, 2005; BRUSCHE, 1983). Somit sind vor allem auf jungen Sukzessionsflächen mit dünnen Trieben und höheren Reisiganteilen auch andere Ergebnisse zu erwarten. Hinsichtlich des GMH 05 soll es aber zum Nachweis reichen, dass sich mit dem Schneckenhackersystem verwertbare Hackschnitzel bei einer vollmechanisierten Mähernte bei der Entbuschung von Offenflächen produzieren lassen. Einen genaueren Bezug der Hackschnitzelqualität zum Ausgangsmaterial wird man aber sicherlich noch herstellen müssen. Eine Zusammenfassung hinsichtlich der Erfüllung bzw. der Möglichkeit zur Erfüllung relevanter Punkte ist in Tab. 6 und Tab. 7 dargestellt. 
Tab. 6: Erfüllung bzw. potenzielle Erfüllbarkeit naturschutzfachlicher Anforderungen nach Kenntnisstand der Vorentwicklung; v : erfüllt; $x$ : nicht erfüllt (+/- : positive/negative Tendenz zur Erfüllbarkeit);

? : bisher nicht sicher abschätzbar

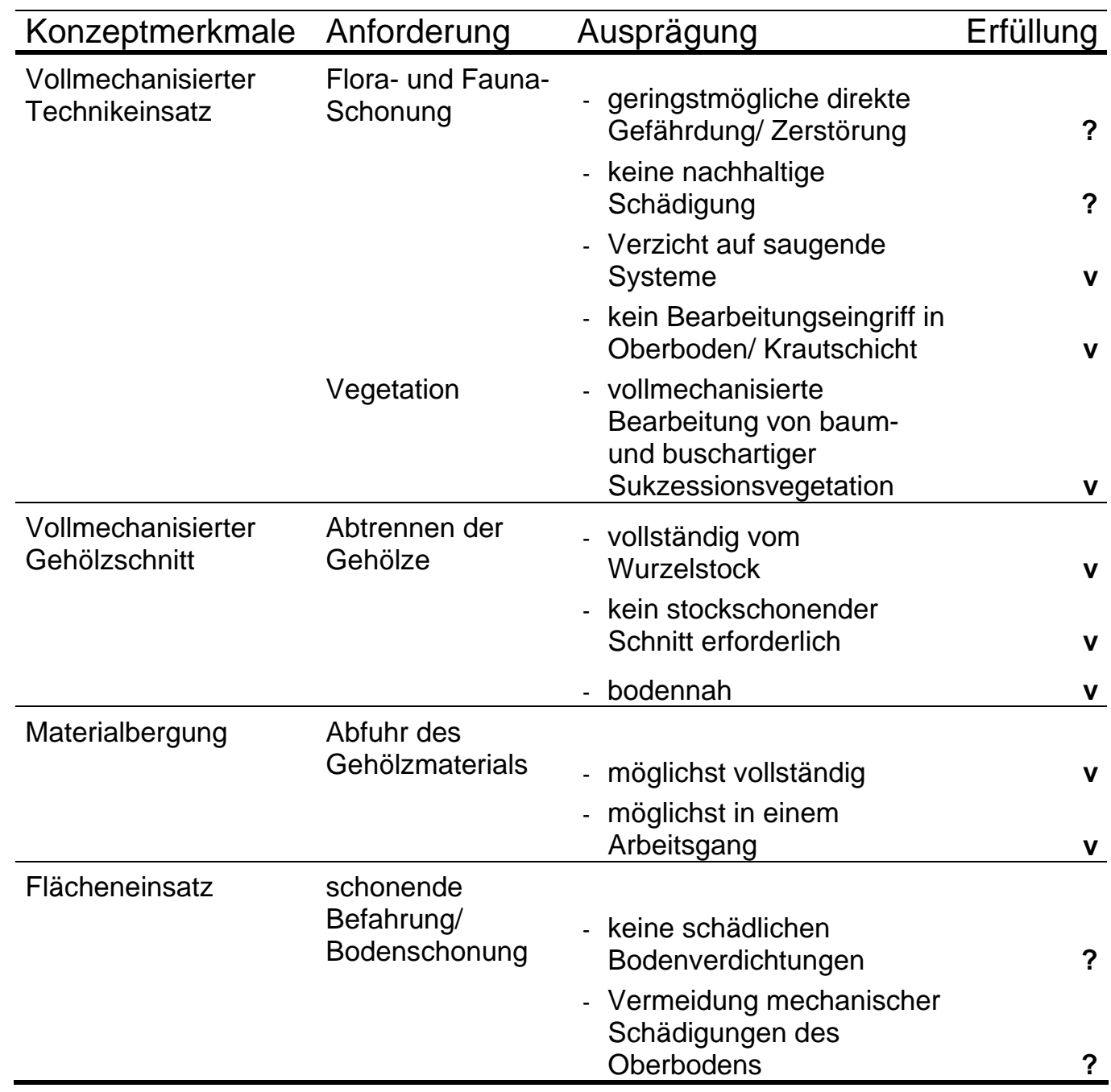


Tab. 7: Erfüllung bzw. potenzielle Erfüllbarkeit technischer und technologischer Anforderungen nach Kenntnisstand der Vorentwicklung; $v$ : erfüllt; $x$ : nicht erfüllt $(+/-$

: positive/negative Tendenz zur Erfüllbarkeit); ? : bisher nicht sicher abschätzbar

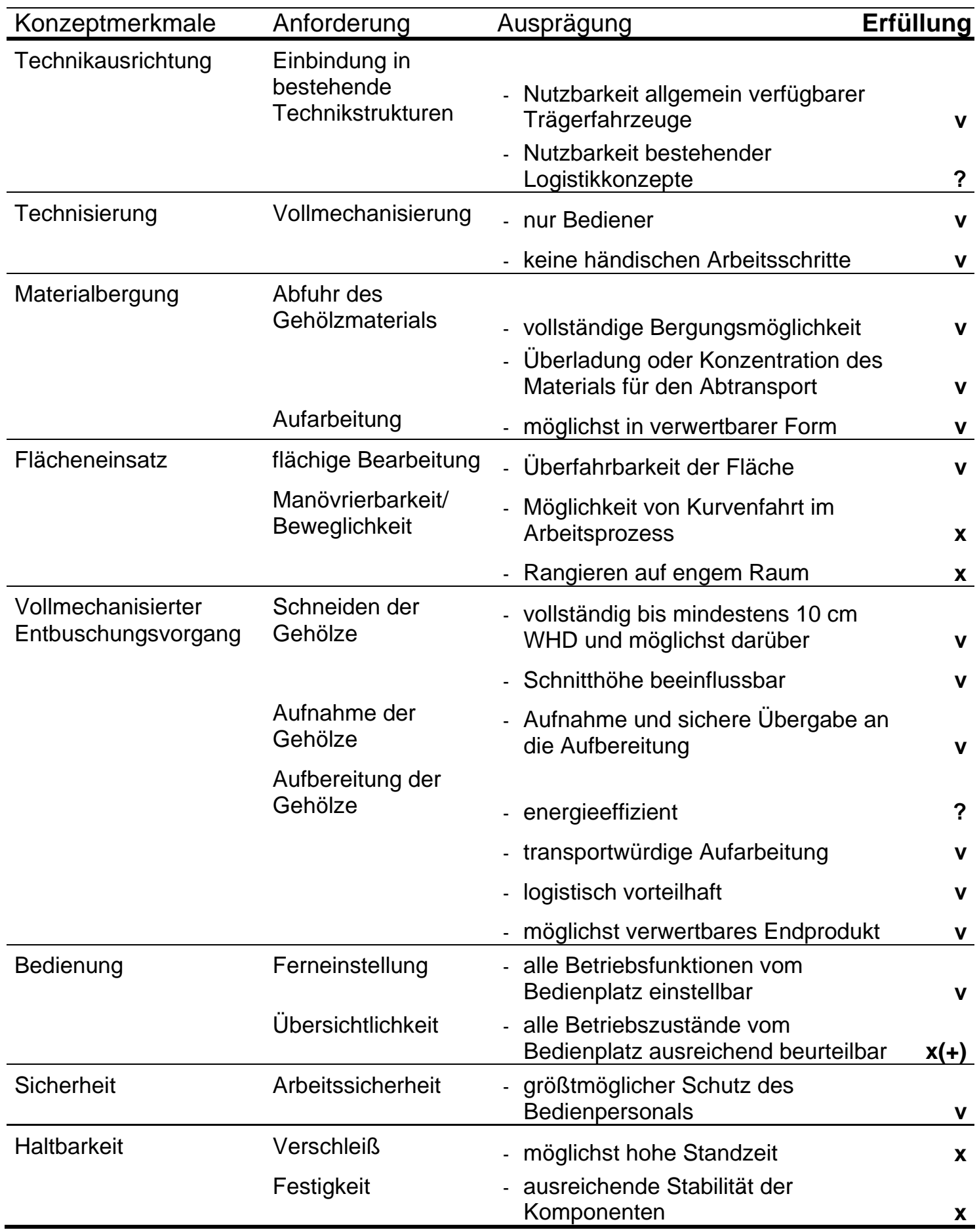




\subsubsection{Zwischenfazit GMH 05}

Das Arbeitsprinzip des Maschinenkonzeptes des GMH 05 kann elementare Anforderungen des Verfahrens zur schlepperbasierten Mähernte von Verbuschungen auf Offenflächen erfüllen. Besonders der waagerechte Einzug der Gehölze und die Trennung der Arbeitschritte Schneiden, Zuführen und Zerkleinern auf separate Maschinenkomponenten können die vollmechanisierte Bearbeitbarkeit der Sukzessionsgehölze ermöglichen. Eine Verwertbarkeit des mit den waagerecht ausgerichteten Häckselrotoren produzierten Materials erscheint prinzipiell möglich.

Die technische Umsetzung des $\mathrm{GMH}$ - Maschinenkonzeptes ist dagegen unbefriedigend gelöst. Es bedarf für eine technische Einsatzfähigkeit weitgehenden Überarbeitungen. Notwendige Optimierungen und vereinzelte Lösungsansätze sind durch den Einsatz des GMH 05 ermittelt, um die Anforderungen in technologischer sowie auch naturschutzfachlicher Hinsicht erfüllen zu können. Diese sind jedoch an dem GMH 05 nicht durchführbar, da sein Aufbau elementar verändert werden muss.

Da die grundlegenden Voraussetzungen für die Erstellung eines Maschinenkonzeptes gefunden scheinen, kann die Vorentwicklungsphase mit der Beendigung der Arbeiten an dem GMH 05 abgeschlossen werden. Die bisherigen Ergebnisse der Maschineneinsätze können nun in der Entwicklungsphase in ein Maschinenkonzept umgesetzt werden.

\subsection{Gehölzmähhäcksler 06}

\subsubsection{Konzeptentwicklung zum GMH 06}

Im Hinblick auf den GMH 05 sind vor allem technische Probleme an dem Maschinenkonzept zu lösen, um die Anforderungen an die Haltbarkeit, den Flächeneinsatz und die Bedienung zu gewährleisten. Deren Lösung kann wiederum auch eine weitere Verbesserung hinsichtlich naturschutzfachlicher Ziele im Bezug auf den Flächeneinsatz und die biotopschonende Bearbeitung darstellen. 
Das Maschinengrundkonzept, wie es beim GMH 05 besteht, soll aus diesem Grund erhalten bleiben und entsprechend der Kernprobleme eine Überarbeitung erfahren.

\subsubsection{Schneiden}

Das Schneiden der Gehölze ist mit dem Sägeblattbalken ausreichend in Triebdurchmesser und Bearbeitungsbreite möglich, weshalb dieser vorerst keiner Änderung bedarf. Beim Vorspannen der Gehölze mit dem Drückbügel zeigt sich, dass eine Verstellung der Ausrichtung in Fahrtrichtung entsprechend der Gehölzvegetation notwendig ist. Aus diesem Grund soll die Bügelausrichtung vom Bediener während des Betriebes situationsangepasst einstellbar sein.

\subsubsection{Aufnehmen und Fördern}

Die nach dem Schneiden erfolgende Aufteilung in zwei Gutströme muss entfallen und eine Bearbeitung des über Maschinenbreite anfallenden Materials in einem Zerkleinerungsaggregat ermöglicht sein. Dieses erfordert eine Konzentration des Materials über die Maschinenbreite zum Häckselaggregat. Zur Konzentration des Materials sowie zur kontrollierten und sicheren Förderung zum Zerkleinerungsaggregat ist eine Verwendung senkrecht stehender Förderwalzen jedoch in dieser Form nicht mehr möglich. Um nur mit einem Gutstrom arbeiten zu können, können maximal zwei Walzen verwendet werden. Diese müssen einen Querförderweg von mindestens einem Meter über die Maschinenbreite zur Konzentration des Materials in der Maschinenmitte ermöglichen. Erst dort ist eine Pressung des Materials zwischen den Walzen und eine Längsförderung möglich. Entsprechend des Querförderweges müsste auch der Durchmesser der Walzen sein. Dieser notwendige Durchmesser führt bei diesen Abmaßen zu einer starken Verlängerung des Zuführbereiches und steht dem Ziel einer Reduktion der Maschinenlänge zuwider. Eine Möglichkeit besteht in der Drehung der Förderachsen um $90^{\circ}$, so dass diese waagerecht und quer zur Fahrtrichtung ausgerichtet sind. So kann eine Längsförderwirkung über eine große Maschinenbreite bei gleichzeitig geringem Förderwalzendurchmesser garantiert werden. Eine Konzentration des Materials kann während des 
Längsfördervorganges zum Häckselaggregat durch einen trichterförmigen Aufbau des Förderkanals erfolgen. Da der Förderweg an der Unterseite der Maschine zwischen Sägeblattbalken und Zuführboden des Zerkleinerungsaggregates geschlossen sein muss und eine gewisse Länge zu erwarten ist, müssen hier entweder aneinander gereihte Walzen oder ein beweglicher Förderboden in Form eines Bandes Verwendung finden. FIRUS \& BELTER (1995b) stellen in ihren Arbeiten zur Förderung von Reisig fest, dass das Erreichen des notwendigen Anpressdruckes eines Fördersystems durch die Pressung auf eine unbewegte Fläche als Widerlager um ein vielfaches schlechter in der Förderleistung zu bewerten ist. Aus diesem Grund wird sich zur Förderung auf angetriebene Komponenten ausgerichtet.

\subsubsection{Zerkleinern}

Die Verwendung des Schneckenhackerprinzips mit waagerecht ausgerichteten Häckselrotoren zur Zerkleinerung soll auch in diesem Maschinenkonzept Verwendung finden, aber auf ein Zerkleinerungsaggregat beschränkt bleiben. Da die Größe der Zuführöffnung einen erheblichen Einfluss auf die Beschickung des Aggregates mit sperrigem Gehölzmaterial hat (BRUSCHE, 1983), muss sie so groß wie möglich gestaltet werden, um eine Verarbeitung des aufkommenden Gesamtmaterials mit einem Häckselrotor zu ermöglichen. Die Zuführöffnung ist beim Schneckenhacker vornehmlich durch den Rotordurchmesser bestimmt (BRUSCHE, 1983). Entsprechend muss ein größerer Häckselrotor verwendet werden, auf den das Material konzentriert wird. Bei der Konstruktion muss jedoch eine Eigenart des Schneckenhackers bei der Zerkleinerung bedacht werden. Die Triebe werden bei der Zerkleinerung entsprechend der Drehrichtung tangential in das Widerlager des Zuführkanals gepresst. Von der Seite des Widerlagers aus ist es während des Zerkleinerungsprozesses deshalb nur schwer möglich weitere Triebe zuzuführen, während eine Zuführmöglichkeit auf der gegenüberliegenden Kanalseite erhalten bleibt (siehe Abb. 53). 


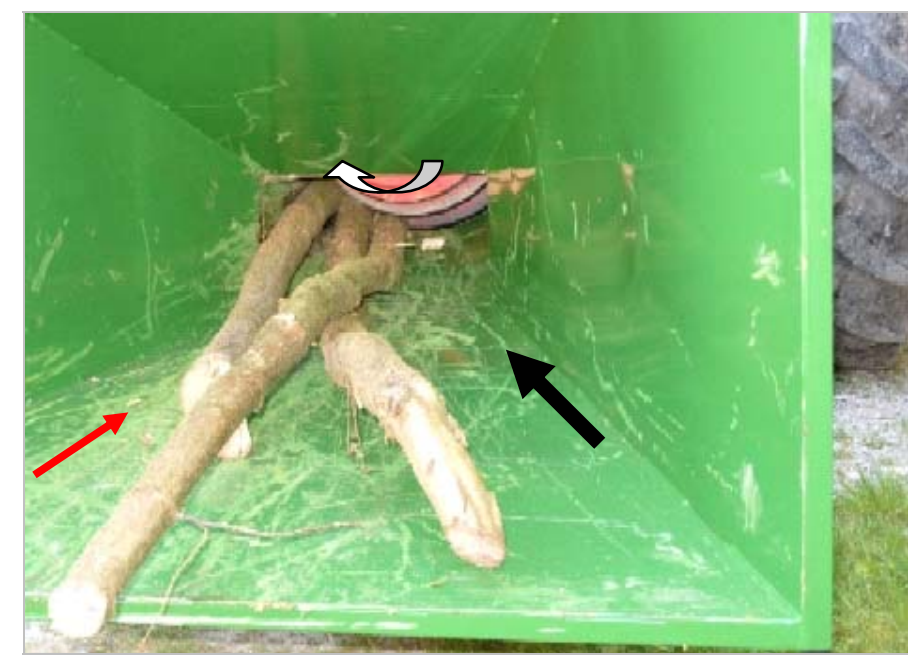

Abb. 53: Blick in den Zuführtrichter eines manuell beschickbaren Schneckenhäckslers bei der Zerkleinerung; weißer Pfeil: Drehrichtung der Häckselschnecke; roter Pfeil: behinderte Zuführrichtung durch Gehölztriebe während der Verarbeitung; schwarzer Pfeil: weitere Zuführung möglich

Um eine Zuführung über die gesamte Kanalbreite und entsprechend aus allen Richtungen der Schneidbreite sicher zu stellen, muss die Zuführung auch von Widerlagerseite aus gewährleistet sein.

\subsubsection{4. Überladen}

Zum Verladen des Materials soll weiter der Wurfbeschleuniger an der Basis des Häckselrotors eingesetzt werden, dabei aber der Übergang in den Überladekrümmer nicht zu einem Verlust an Wurfleistung führen. Gleichzeitig ist es für die Übersicht in den Arbeitsbereich erforderlich, das der Krümmer seitlich aus dem Blickfeld des Bedieners gerückt wird. Auf den Einsatz des Kamerasystems soll trotzdem nicht verzichtet werden, da aus der Bedienerposition prinzipiell keine Einsehbarkeit des Häckselbereiches ermöglicht werden kann.

\subsubsection{Antriebskonzept}

Der Antriebsstrang bei dem Maschinenkonzept muss vor möglichen Wechsellasten geschützt sein oder diese durch dessen Konstruktion vermeiden. Die Verwendung nur eines Häckselrotors ermöglicht den direkten Antrieb über die Schlepperzapfwelle, wenn der Häckselrotor maschinenmittig 
positioniert wird. Da die Zapfwelle parallel zur Rotorachse liegt und eine gleiche Drehrichtung am Häckselrotor eingesetzt werden kann, kann der Zerkleinerungsantrieb ohne jegliches Getriebe auskommen. Dieses wird zudem Gewicht und Baulänge einsparen.

Der Sägeblattantrieb stellt die nächste Komponente mit einem hohen Leistungsbedarf dar, deren Antrieb jedoch nicht formschlüssig oder zumindest gedämpft erfolgen soll, um die Übertragung bzw. das Entstehen von Drehmomentspitzen zwischen Komponenten zu vermeiden. Bei mechanischen Antrieben kann dieses nur durch den Einbau von Dämpfungselementen oder z.B. über Riementriebe erfolgen. Die Auslegung von Dämpfungselementen gestaltet sich jedoch als schwierig, da sie nur für bestimmte Betriebszustände ( -bereiche) anpassbar sind und in ungünstigen Fällen schädliche Resonanzschwingungen erzeugen können (SCHMIDT, 2006). Riemengetriebe erreichen eine dämpfende Wirkung durch Längsdehnung der Riemen und durch Riemenschlupf, wobei vor allem letzterer sich stark auf die Lebensdauer der Komponenten auswirkt (SCHLECHT, 2010). BLOCK (2000) berichtete von diesem Problem bei der Konzeption der ersten Tritucap- Antriebe. Hydrostatische Antriebe sind hier offensichtlich die günstigste Lösung, um den Antrieb schwingungsarm gestalten zu können (GROTE ET AL., 2007). Da die notwendige Hydraulikleistung nicht durch den Schlepper zur Verfügung gestellt werden kann, da diese zur Steuerung der Maschine benötigt wird, muss ein maschineneigenes Hydrauliksystem mit Öltank, Pumpen, Filtern, Kühlern etc. installiert werden. Gleichzeitig können auch die Antriebe der Zuführung über dieses System permanent versorgt werden. Da sich hydrostatische Antriebe allgemein und vor allem am Ort der Leistungsübertragung sehr platzsparend installieren lassen sowie sich die Kraftübertragungsleitungen (Schläuche, Rohre) sehr flexibel installieren lassen (GROTE ET AL., 2007), erweitern diese die baulichen Möglichkeiten im Arbeitsbereich.

\subsubsection{Arbeitsprinzip}

Das allgemeine Arbeitsprinzip des neu entwickelten GMH 06 ist in Abb. 54 zu sehen und unterscheidet sich verfahrenstechnisch nur unwesentlich von den 
vorherig beschriebenen Prototypen. Das Vorspannen der Triebe, das Fällen, das Aufnehmen, das Zerkleinern und das Überladen der Hackschnitzel soll auch hier in Schubfahrt und in einem Arbeitsgang erfolgen. Die vornehmlichen Änderungen finden im technischen Bereich des Maschinenkonzeptes statt.

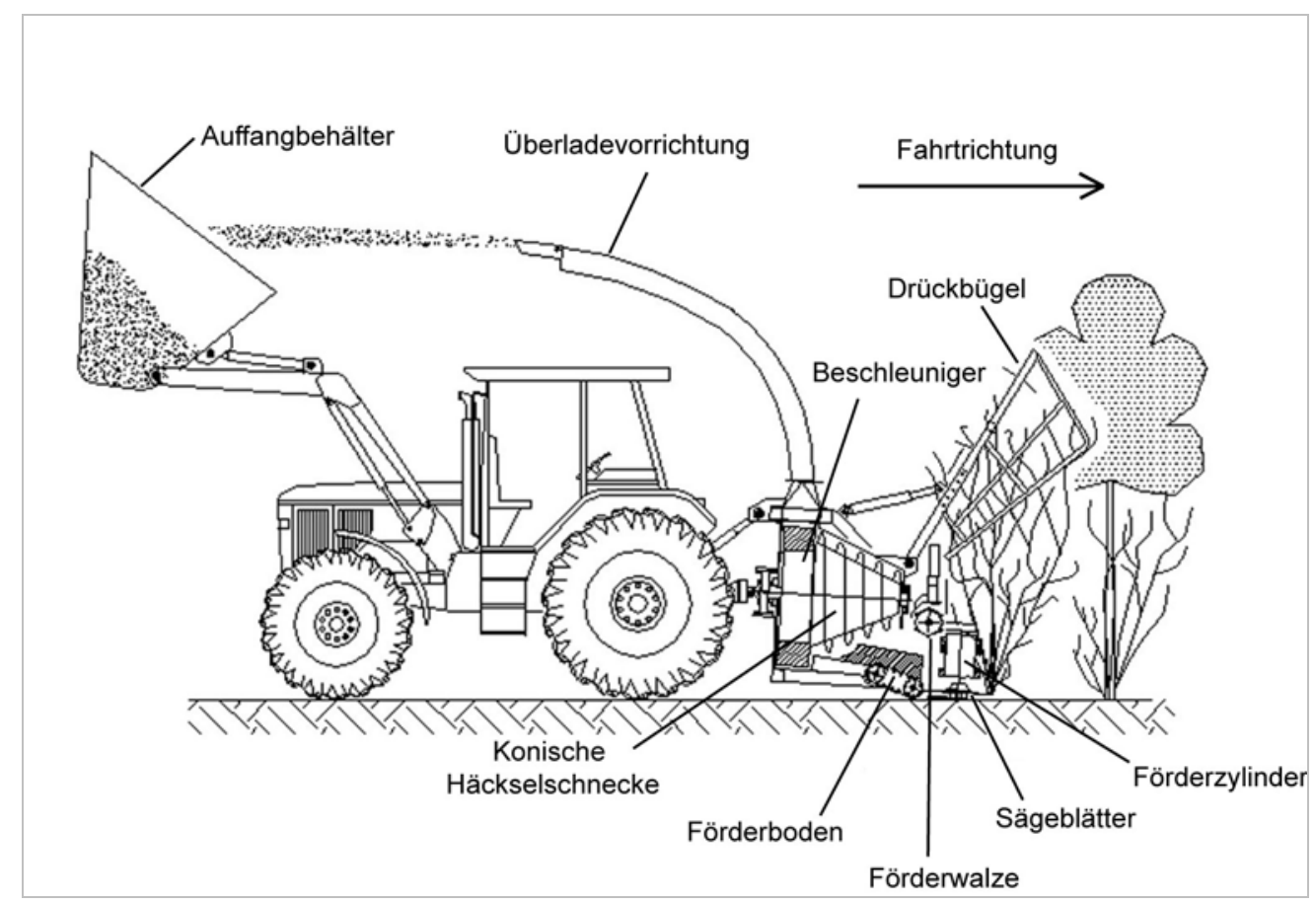

Abb. 54: Arbeitsprinzip des Gehölzmähhäckslers 06 bei der vollmechanisierten Entbuschung

\subsubsection{Umsetzung des Maschinenkonzeptes GMH 06}

Durch den geänderten Aufbau kann die Maschine im Gegensatz zum GMH 05 auf 2,1 m von den Unterlenkerkoppelpunkten des Schleppers aus verkürzt werden und erreicht nach der Fertigstellung ein Gewicht von $2 \mathrm{t}$. 


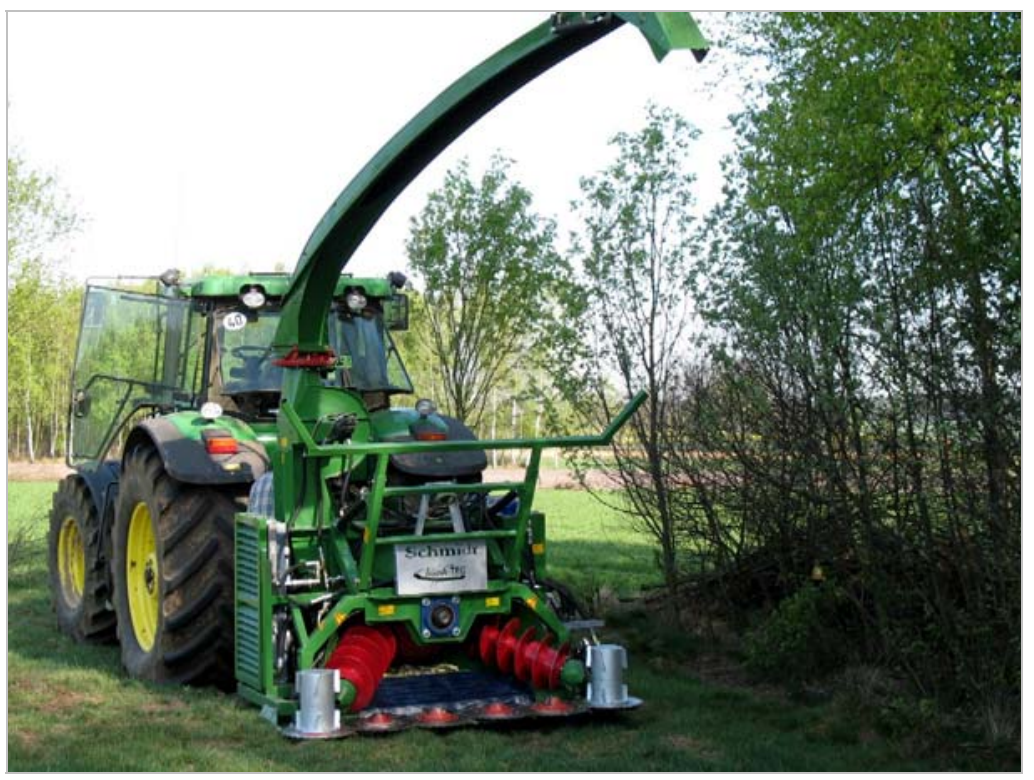

Abb. 55: Gehölzmähhäcksler 06 im Frühjahr 2007 nach der Fertigstellung

Die maximale Breite der Maschine ist wieder durch die Arbeitsbreite des Sägeblattbalkens bestimmt. Die wesentlichen technischen Merkmale des GMH 06 werden im Folgenden erläutert. In Abb. 56 ist der prinzipielle technische Aufbau dargestellt. 


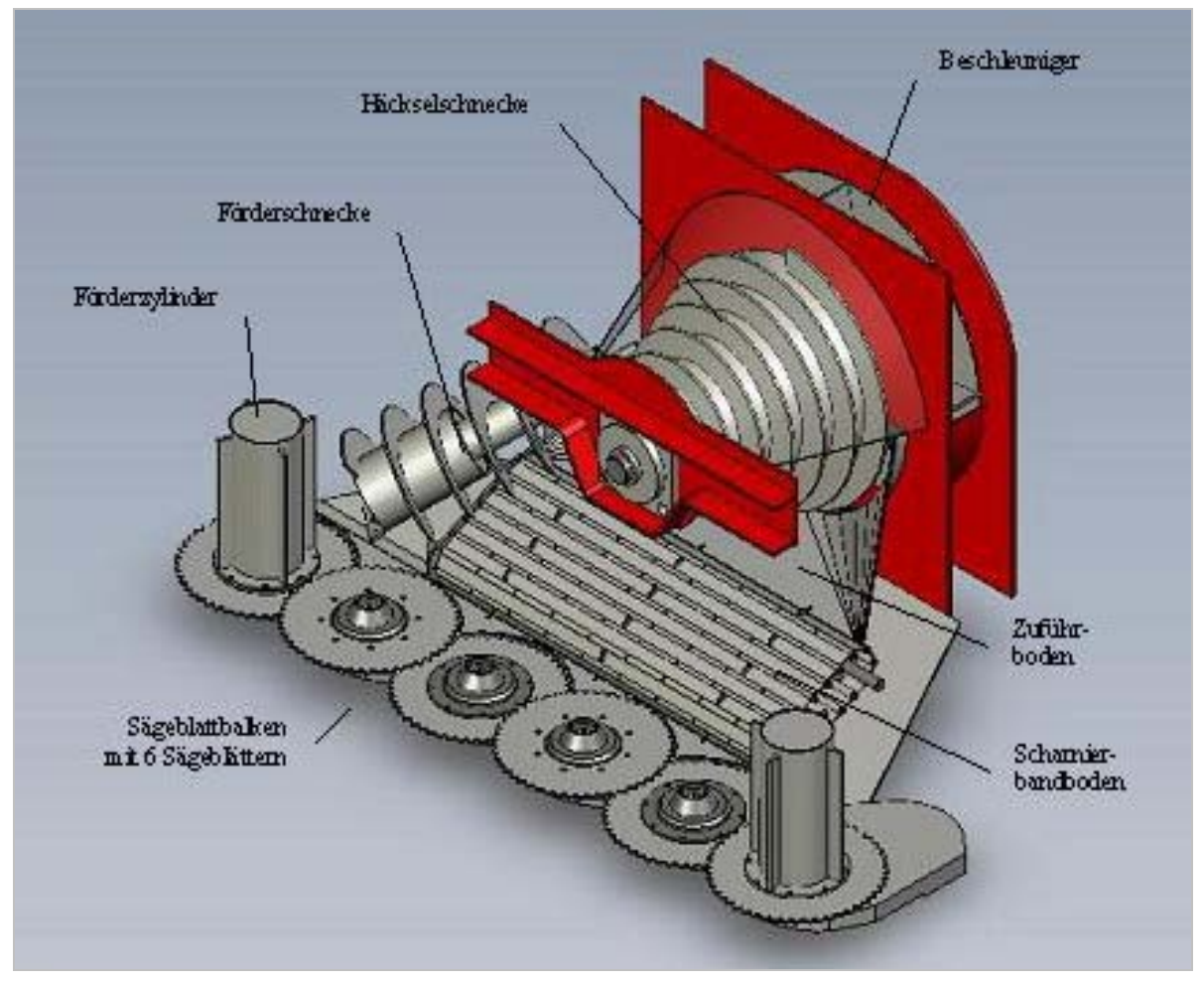

Abb. 56: Konstruktionszeichnung des Gehölzmähhäckslers 06 (vereinfachte Darstellung mit einer Förderschnecke, ohne Förderwalze, Auswurfturm und Drückbügel)

\subsubsection{Schneiden}

Die Schneidgarnitur wird baugleich vom GMH 05 übernommen, jedoch wird der mechanische Antrieb durch einen hydrostatischen ersetzt. Dazu wird anstelle des Winkelgetriebes ein leistungsstarker Ölmotor adaptiert. Der Ölmotor kann laut des installierenden Unternehmens eine Leistung von $55 \mathrm{~kW}$ aufbringen, wobei diese annähernd an der vom Hersteller des Sägeblattbalkens zulässigen Antriebsleistung liegt.

Um die Gehölze vor dem Schneiden vorzuspannen, wird auf dem GMH 06 ein hydraulisch verstellbarer Drückbügel in Form einer Gabel angebracht, die die Bearbeitungsbreite abdeckt. Der Bügel kann in Fahrtrichtung geschwenkt werden, um den Druck beim Vorspannen zu erhöhen bzw. die Triebe schon vor dem Schneiden in eine waagerechtere Position zu bringen. Zudem kann er mechanisch teleskopiert werden, um inn je nach vorliegenden Bedingungen auf eine vorteilhafte Länge einzustellen. 


\subsubsection{Aufnehmen und Zuführen}

Zur Förderung der geschnittenen Gehölztriebe zum Häckselaggregat werden mehrere Förderaggregate integriert. Einerseits werden hinter dem Sägeblattbalken diagonal zur Fahrtrichtung verlaufende Förderschnecken angebracht, welche die Triebe bei gleichzeitiger Längsförderung zum Häckselaggregat auch zur Maschinenmitte hin konzentrieren sollen und andererseits ein Scharnierbandboden, welcher mittig hinter dem Sägeblattbalken über die Hälfte der Maschinenbreite reicht.

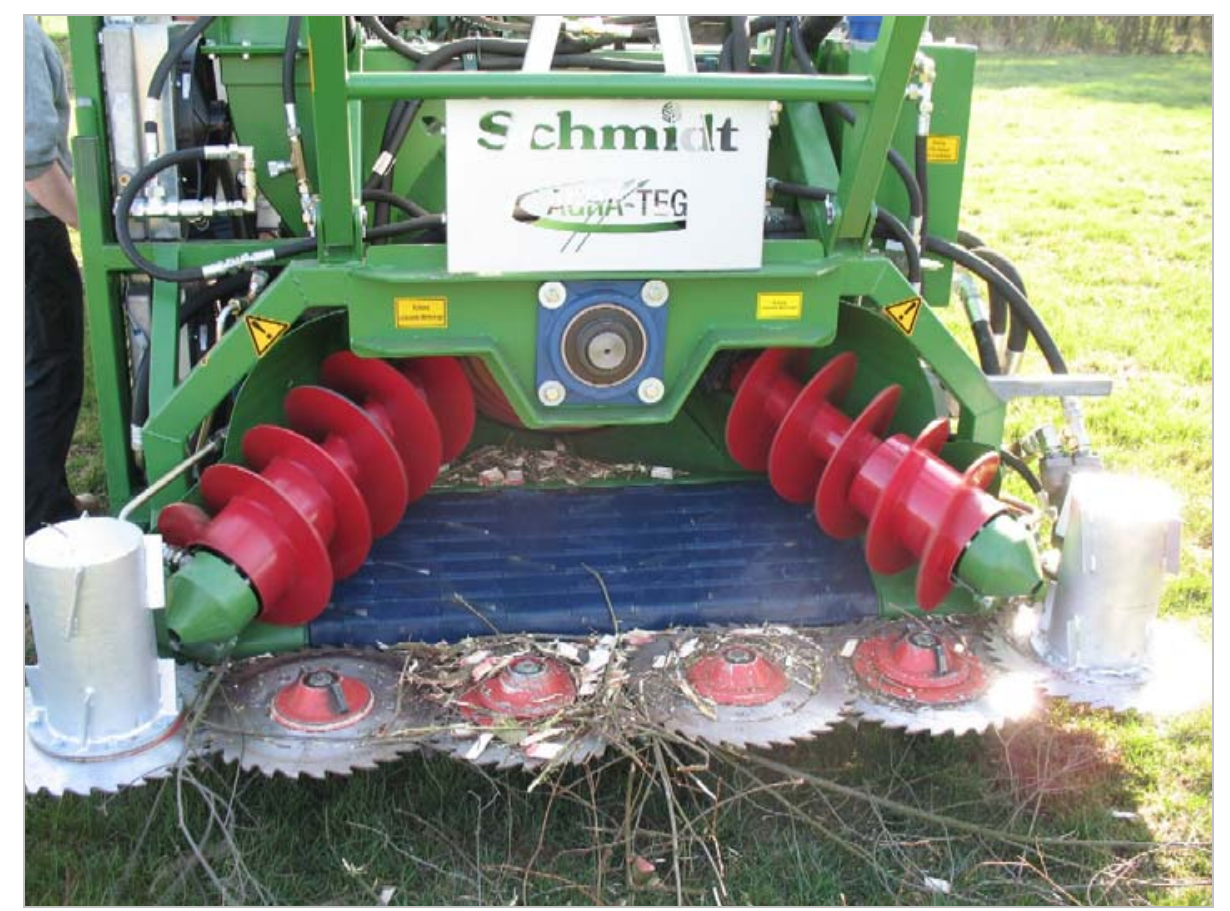

Abb. 57: Blick in den Arbeitsbereich des Gehölzmähhäckslers 06 mit Zuführschnecken (rot), Scharnierbandboden (blau) und Förderzylindern (silber)

Der Scharnierbandboden ist aus Kettenleisten mit einer Breite von $1200 \mathrm{~mm}$ und einer Länge von $52 \mathrm{~mm}$ aufgebaut. Insgesamt deckt er eine Förderstrecke von 500 mm von der Rückseite des Sägeblattbalkens bis unter die Häckselschnecke ab. Zur Erhöhung der Griffigkeit ist er mit diagonal zur Förderrichtung ausgerichteten Stegen aus $10 \mathrm{~mm}$ Quadratstahl besetzt. Die diagonale Anordnung wird gewählt, um ein Einklemmen des Materials am Ende der Förderstrecke zu verhindern, wo das Scharnierband an einer stählernen Abstreiflippe unter den Zuführboden läuft. 
Um das geschnittene Material von den äußeren Sägeblättern zur Maschinenmitte zu bewegen, werden auch beim GMH 06 Förderzylinder zentrisch auf die äußeren Sägeblätter montiert.

Die Förderung der geschnittenen Gehölztriebe erfolgt somit seitlich durch die Förderzylinder und nachfolgend durch die Zuführschnecken sowie am Boden durch den Scharnierbandboden. Um das meist voluminöse und sparrige Material auch von der Oberseite der Zuführöffnung einer Förderbewegung auszusetzen, wird eine sogenannte Förderwalze quer zur Förderrichtung über den Übergang vom Sägeblattbalken zum Scharnierbandboden eingerichtet. Sie hat eine Breite von $1000 \mathrm{~mm}$ und deckt somit in diesem Bereich den Abstand zwischen den beiden Zuführschnecken ab. Sie ist in Abb. $58 \mathrm{zu}$ sehen und ist anfangs fest eingebaut. Im Nachhinein wird sie hydraulisch höhenverstellbar ausgeführt, um die mit $10 \mathrm{~mm}$ hohen und $20 \mathrm{~mm}$ breiten Förderelementen bestückte Walze je nach Mengenaufkommen von oben auf das Fördergut andrücken zu können.

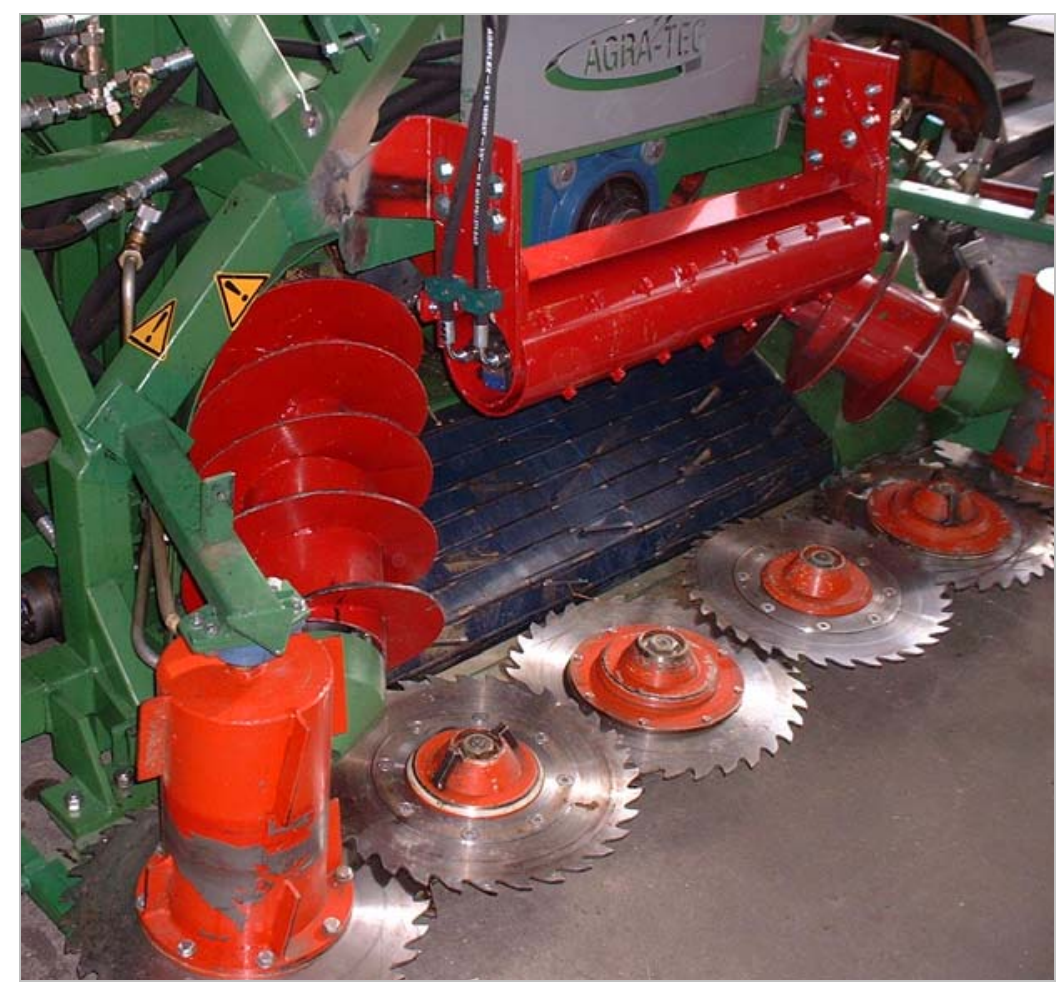

Abb. 58: Blick auf die Schneid- und Zuführkomponenten des Gehölzmähhäckslers 06. Über dem Übergang von Sägeblattbalken und Scharnierbandboden (blau) ist eine hydraulisch angetriebene Förderwalze installiert. 


\subsubsection{Zerkleinern}

Das Häckselaggregat des GMH 06 ist nach dem Schneckenhackerprinzip ausgeführt, besteht jedoch nur noch aus einem maschinenmittig positionierten Häckselrotor. Im Gegensatz zum GMH 05 wird der Konuswinkel von $10^{\circ}$ auf $22^{\circ}$ und der Rotordurchmesser von 700 auf $780 \mathrm{~mm}$ erhöht. Dieses hat zum Zweck, die Einzugsöffnung des Häckselrotors zu vergrößern. Die Schneide des Häckselrotors ist eingängig ausgeführt und hat einen Windungsabstand von $80 \mathrm{~mm}$. Die Windungshöhe beträgt ebenfalls $80 \mathrm{~mm}$ und die Materialstärke wird mit $8 \mathrm{~mm}$ größer gewählt als beim GMH 05. Rechnerisch ergibt sich bei diesem Aufbau der Häckselschnecke eine Einzugsgeschwindigkeit der Schnecke von $0,72 \mathrm{~m} / \mathrm{s}$ bei $540 \mathrm{U} / \mathrm{min}$ und $1,33 \mathrm{~m} / \mathrm{s}$ bei $1000 \mathrm{u} / \mathrm{min}$.

Der Häckselspalt wird unterhalb der Häckselschnecke durch den Zuführboden als radiales und durch drei zusätzliche Anschläge als tangentiale Widerlager für das zu zerkleinernde Material gebildet. Die zusätzlichen Anschläge sind als Dreiecke ausgeformt und dem Konus angepasst. Auf diese Weise ergeben sich drei zusätzliche Punkte, an denen Material tangential fixiert und an diesen Punkten bei der Zerkleinerung eingezogen werden kann (Abb. 59). 


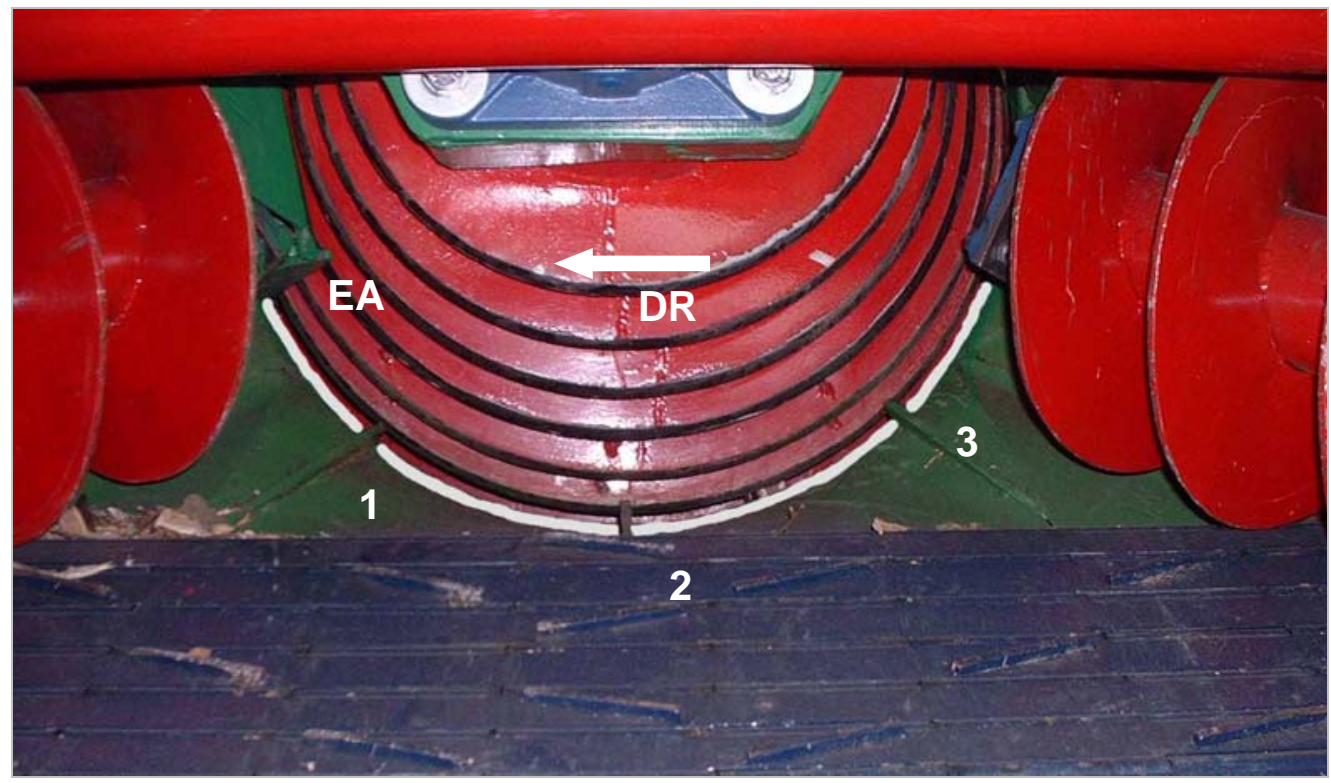

Abb. 59: Blick in den Häckselspalt des Gehölzmähhäckslers 06 Weiße Linie: Arbeitsbereich des Häckselspaltes

EA: Endanschlag

DR: Drehrichtung

1, 2 und 3:zusätzliche Häckselpunkte, die durch Anschläge

(Dreiecke) gebildet werden.

\subsubsection{4. Überladen}

Das zerkleinerte Material, die Hackschnitzel, werden auch bei diesem Prototyp mit einem Wurfgebläse übergeladen. Dazu werden die Hackschnitzel mit einem Beschleuniger, der als Teil des Häckselrotors direkt an der Basis der Häckselschnecke integriert ist, mit der notwendigen kinetischen Energie versorgt. Er ist somit auf der gleichen Lagerungswelle angebracht und erhält durch die feste Verbindung die gleiche Drehzahl wie die Häckselschnecke. Der Beschleuniger besteht aus vier Auswurfschaufeln, die mit einem Durchmesser von $900 \mathrm{~mm}$ rotieren. Bei einer Drehzahl von $540 \mathrm{U} / \mathrm{min} \quad(1000 \mathrm{U} / \mathrm{min})$ erreicht der Beschleuniger eine Umfangsgeschwindigkeit von $25,45 \mathrm{~m} / \mathrm{s}(47,12 \mathrm{~m} / \mathrm{s})$.

Die Hackschnitzel werden nach der Zerkleinerung axial in den Auswurfrotor gefördert, von den Auswurfschaufeln tangential beschleunigt und verlassen durch die entstehende Fliehkraft am senkrecht nach oben mündenden Auslassschacht den Beschleuniger zum Auswurfkrümmer. Der Krümmer ist auf dem Auslassschacht angebracht und kann stufenlos um $360^{\circ}$ mit einem 
Hydraulikmotor verdreht werden, der über ein doppeltwirkendes Hydrauliksteuergerät des Schleppers angetrieben wird. Durch die seitlich versetzte Positionierung steht er nicht mehr im Blickfeld auf den Arbeitsbereich. Der Auswurfkrümmer lenkt den Gutstrom von senkrechter Förderung in die waagerechte um. Am Ende des Krümmers ist ein Prallblech angebracht, welches vom Schlepper aus hydraulisch und stufenlos um $90^{\circ}$ verstellt werden kann. Der waagerechte Gutstrom kann so am Ende des Krümmers von der waagerechten Förderung wieder bis zur senkrechten umgeleitet werden, so dass ein waagerechtes Werfen oder eine Beladung auf eine Transportmöglichkeit von oben erfolgen kann. Die Mündung des Krümmers befindet sich in Arbeitsposition der Maschine im Radius von 3,2 m um den Drehpunkt und ca. 3,9 m über dem Boden.

Zur Bergung wird weiterhin der Überladecontainer im Frontlader eingesetzt. An inm sind keine Umbauten notwendig.

\subsubsection{Antriebskonzept}

Der Antrieb des GMH 06 wird im Gegensatz zum GMH 05 umfangreich verändert. Er besteht aus einem mechanischen Hauptantrieb, welcher die Schlepperleistung auf das Häckselaggregat sowie auf den Antrieb einer hydraulischen Eigenversorgung der Maschine überträgt. Dabei erfolgt der mechanische Antrieb des GMH 06 über den Zapfwellenanschluss des Schleppers stark vereinfacht im Vergleich zum GMH 05. Die Gelenkwelle ist direkt über eine Überlastkupplung (Sicherheitsreibkupplung mit $3000 \mathrm{Nm}$ Lösemoment) mit der Lagerungswelle des Häckselrotors verbunden und treibt diesen somit direkt an. Die Drehzahl der Häckselschnecke entspricht somit auch der vom Schlepper abgegebenen Zapfwellendrehzahl. An der schlepperseitigen Lagerung des Häckselrotors wird ein Keilriemenantrieb angebracht, über den eine Dreifach- Hydraulikpumpe betrieben wird. Diese stellt das Kernstück der hydraulischen Eigenversorgung des GMH 06 dar (Abb. 60). Die erste Pumpe versorgt den Ölmotor des Sägeblattantriebes, welcher nun hydrostatisch betrieben wird. Die Drehzahl lässt sich durch einen hydraulischen Mengenteiler auf die erforderliche Drehzahl von ca. $2350 \mathrm{U} / \mathrm{min}$ einstellen. Die zweite Pumpe treibt die beiden Ölmotoren der 
Zuführschnecken an und die dritte die Ölmotoren der Förderwalze sowie des Scharnierbandbodens.

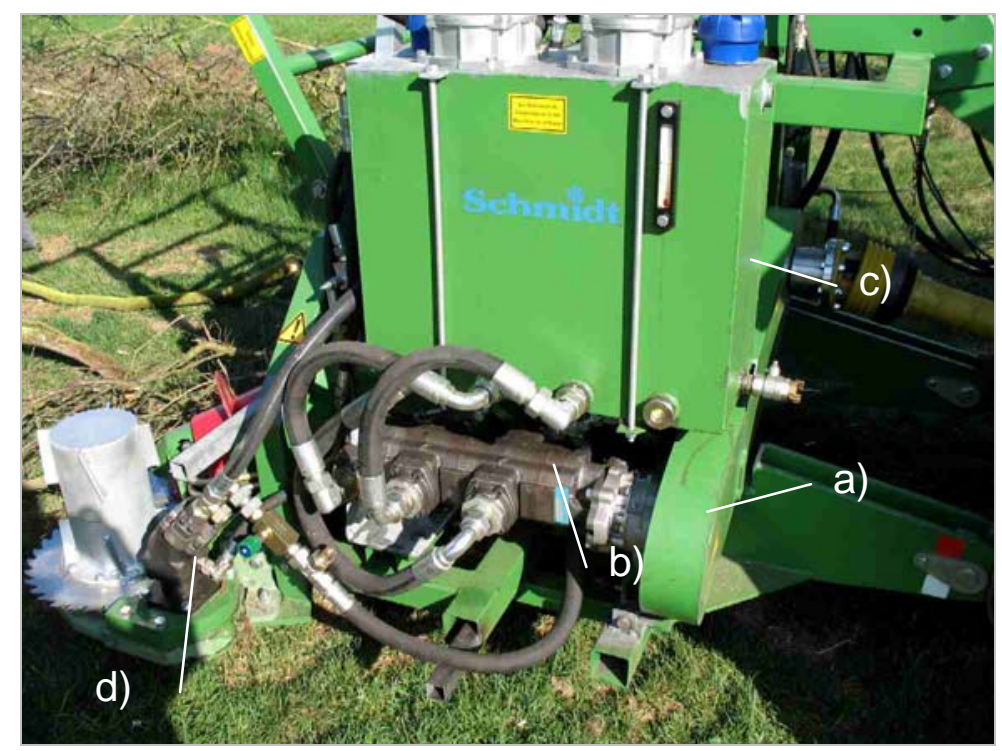

Abb. 60: Hydraulische Eigenversorgung des

Gehölzmähhäckslers 06 mit a) Keilriemenantrieb; b)

Dreifachpumpe; c) Öltank (100 Liter) mit Ölfiltern; d) Ölmotor des Sägeblattantriebs

Das notwendige Hydrauliköl wird mit einem 100 Liter fassenden Öltank mitgeführt, welcher linksseitig ${ }^{4}$ der Maschine über den Pumpen positioniert ist. Rechtsseitig werden zwei Hydraulikölkühler eingebaut, die die Überschusswärme des sich im Betrieb erhitzenden Hydrauliköls abführen. Die elektrische Stromversorgung der Kühlerventilatoren wird über die Bordelektronik des Schleppers sichergestellt. In Abb. 61 ist der gesamte mechanische Antrieb und die hydraulische Eigenversorgung skizziert abgebildet. Vor allem der Hydraulikkreislauf ist stark vereinfacht dargestellt.

\footnotetext{
${ }^{4}$ Positionsangaben an der Maschine sind, wenn nicht näher erläutert, immer aus der Draufsicht in Arbeitsrichtung (Fahrtrichtung) der Maschine angegeben (Sägeblattantrieb befindet sich z.B. vorne).
} 


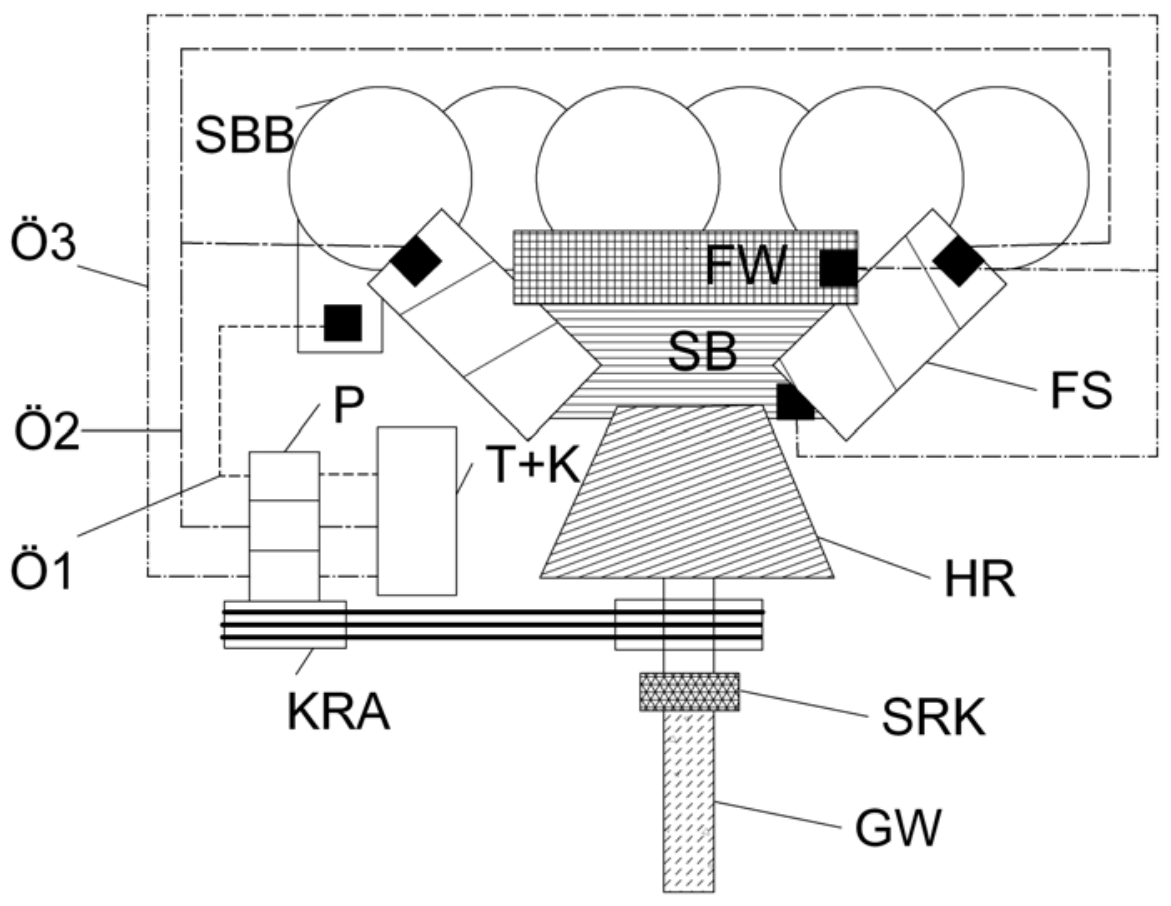

Abb. 61: Antriebskonzept des Gehölzmähhäckslers 06 (skizziert, dabei sind Ölkreisläufe nur angedeutet und nicht detailgetreu dargestellt); mit:
Ö: Ölkreisläufe
$P$ : Dreifach- Pumpe
T+K: Tank und Kühler
FW: Förderwalze SBB: Scharnierbandboden
KRA: Keilriemenantrieb
FS: Förderschnecke
HR: Häckselrotor
GW: Gelenkwelle
: Ölmotoren

SRK: Rutschkupplung

Die in der Konzeptentwicklung empfohlene Reversiereinrichtung zur Behebung von Blockaden wird während der Konstruktion diskutiert und auch mehrere technische Lösungsvorschläge dazu unterbreitet. Zur Lösung werden z.B. elektrisch oder hydrostatisch angetriebene Motoren angedacht, die ähnlich eines Anlassers mit großer Untersetzung und entsprechend hohem Drehmoment am Beschleunigerumfang eine Reversierung ermöglichen können.Des Weiteren wird der Einbau eines hydraulisch oder mechanisch absenkbareren Zuführbodens angedacht. Die Umsetzung dieser Lösungen ist jedoch technisch sehr aufwendig zu implementieren, so dass in dieser Entwicklungsstufe vorerst auf die Umsetzung verzichtet wird. Der Fokus kann so verstärkt auf die Funktionsfähigkeit des Gesamtkonzeptes der Maschine gelegt werden. Um Störungen bei dieser Maschine dennoch beseitigen zu können, wird eine Vorrichtung zur manuellen Reversierung des Rotors per Hebelkraft eingerichtet. 


\subsubsection{Mobileinheit}

Der GMH 06 wird in der Kombination mit einem landwirtschaftlichen Schlepper als Basisfahrzeug belassen und mit dem Frontladercontainer zur Bergung des Hackschnitzelmaterials ausgestattet.

\subsubsection{Praxiseinsätze des GMH 06}

Die Praxiseinsätze erfolgen wieder auf den beschriebenen Versuchsflächen, auf denen unterschiedlichste Kombinationen der Vegetationsstrukturen bearbeitet werden.

\subsubsection{Schneiden}

Tests zum Schneidvermögen des nun hydrostatisch angetriebenen Sägeblattbalkens können aufgrund der gleichen Erfahrungen wie beim GMH 05 nicht über $12 \mathrm{~cm}$ WHD durchgeführt werden, da der dann aufkommende Getriebekasten des Mähbalkens in der Position nur unwesentlich verändert ist. Die Leistung des verwendeten Antriebes stößt jedoch beim Auftreten einer zu großen Gesamtschnittfläche durch mehrere gleichzeitig zu schneidende Triebe an ihre Grenzen und kommt aufgrund des zu hohen Leistungsbedarfes zum Stillstand.

Um so dicker die Triebe werden, umso geringer darf ihre Anzahl sein, was sich letztlich durch die resultierende zu bearbeitende Gesamtschnittfläche begründen lässt. Schneidversuche zeigen, dass sich leistungsbezogen bis zu drei Triebe im Bereich von 10- $12 \mathrm{~cm}$ Schnittflächendurchmesser gleichzeitig durchtrennen lassen. Dieses entspricht ungefähr einer gleichzeitig geschnittenen Gesamtfläche von 236- $339 \mathrm{~cm}^{2}$. Der Versuch den Bezug zwischen Schneidvermögen, Schnittfläche und Triebzahl messtechnisch zu quantifizieren, scheitert jedoch bei den Untersuchungen. Einerseits ist es vor allem bei einer Vielzahl dünner Trieben nur schwierig möglich, diese gleichzeitig zu schneiden und andererseits vollzogen die Sägeblätter durch die Überlappung teilweise einen Doppelschnitt. Dadurch ist die reale Schnittfläche stark abweichend von der Schnittfläche der Triebstümpfe. Nach FELDE (1980) soll bei einer derartigen Schnittfläche die benötigte Leistung ca. $2 \mathrm{~kW}$ betragen. Dieses liegt weit unter der zur Verfügung stehenden 
Leistung von $55 \mathrm{~kW}$. Auch wenn keine Werte bezüglich des Wirkungsgrades des Getriebes vorliegen, muss der Einfluss durch Doppelschnitte und reibendem Material zwischen den überlappenden Sägeblättern einen hohen Leistungsbedarf erfordern. Technische Ausfälle des Antriebes oder des Sägeblattbalkens sind während der Einsätze nicht zu verzeichnen.

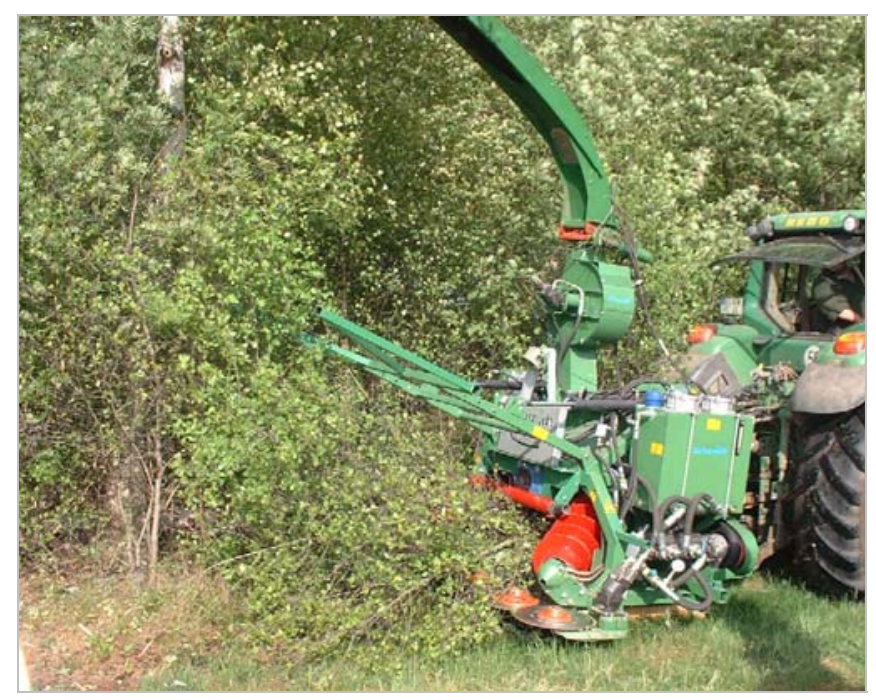

Abb. 62: Gehölzmähhäcksler 06 bei Ersteinsätzen in Birkensukzession am Waldrand

Mit dem hydraulisch einstellbaren Drückbügel kann beim GMH 06 die notwendige Vorspannung der Triebe und das waagerechte Ausrichten situationsangepasst vom Schlepper aus eingestellt werden. Wie beim GMH 05- Einsatz zeichnen sich hier aber auch Grenzen der Bearbeitbarkeit ab. In dichten und sehr dichten Beständen sowie auch bei dicken WHD weniger Triebe kann die notwendige Vorspannung für das Ausrichten der Triebe und auch der begünstigende Schleudereffekt in den Einzug nicht mehr erreicht werden. Die Flexibilität der Triebe, um eine Spannung bzw. Biegung der Gehölze aufzubauen, nimmt mit zunehmendem WHD ab. Gleichermaßen verhält es sich bei der baum- und buschartigen Wuchsstruktur. Buschartige Gehölze lassen sich in dichten Beständen entgegen den baumartigen schon bei geringerem WHD nicht mehr durch die in Arbeitsrichtung stehende Vegetation waagerecht ausrichten. Sie können nicht zwischen den Kronen hindurchgedrückt werden und stützen sich auf der folgenden Vegetation stark ab. Baumartige lassen sich durch den schlankeren Wuchs auch bis in dichte oder sehr dichte Bestände 
vorspannen, solange sie ausreichend flexibel sind und die Stabilität durch den Bestand nicht zu groß wird. So muss z.B. bei der Vegetation in Abb. 63 die Entbuschungsarbeit abgebrochen werden, da die sehr dichte und verwachsene Weißdornvegetation einen zu großen Widerstand darstellt. Eine waagerechte Ausrichtung ist dort nicht möglich, obwohl die dort vorliegenden Gehölzstärken bei WHD von $4-6 \mathrm{~cm}$ in lichteren Beständen ohne Weiteres bearbeitet werden können. In diesem Fall determinieren die Bestandesdichte und die Wuchsform der Gehölze das Entbuschungsvermögen.

Insgesamt sind die Tendenzen dieses Zusammenhangs gleich den Beobachtungen beim GMH 05, dabei erreicht der GMH 06 durch den längeren und hydraulisch verstellbaren Bügel bessere Arbeitsergebnisse. Es kann mit ihm auch an Stellen weitergearbeitet werden, wo aufgrund der Bestandesdichte und vor allem der Bestandeshöhe die Entbuschung mit dem GMH 05 nicht mehr möglich ist.

Durch das verstärkte Arbeiten mit dem GMH 06 in sehr dichten Beständen zeigt sich zudem eine grundsätzliche Schwäche des Mäherntesystems. Die seitliche Trennung von verwachsenem Gehölzmaterial ist nicht immer möglich. Vor allem Büsche neigen dazu, seitlich in den Bearbeitungsbereich hineinzuhängen und auch mit dem zu bearbeitenden Material ineinander verwachsen zu sein. Eine einfache Trennung durch Separieren mit Hilfe des Drückbügels ist dort nicht mehr möglich. 


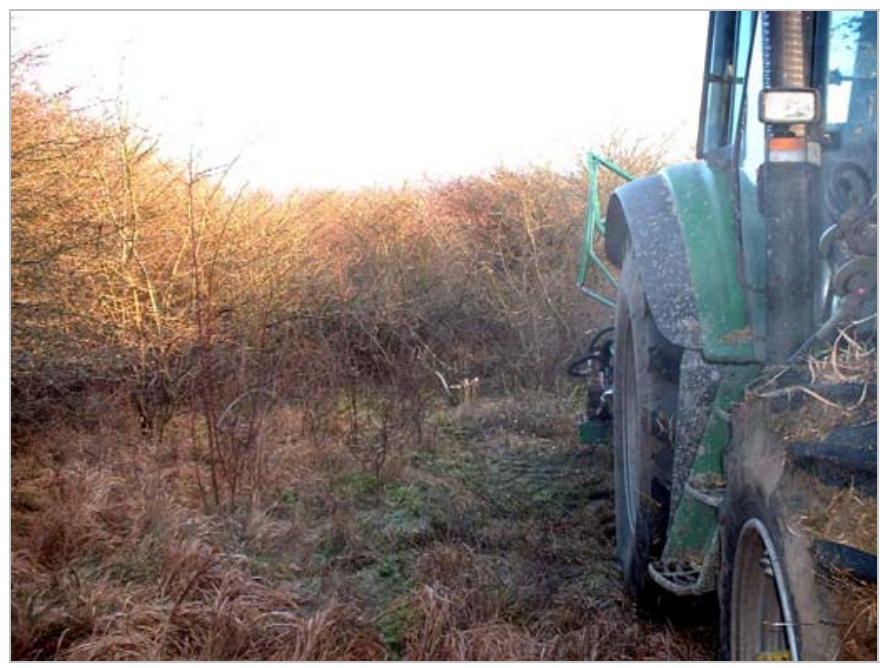

Abb. 63: Abbruch der vollmechanisierten Entbuschung mit dem Gehölzmähhäcksler 06 durch eine zu hohe Bestandesdichte in buschartiger Vegetation mittleren Wurzelhalsdurchmessers (6$8 \mathrm{~cm}$ WHD)

\subsubsection{Aufnehmen und Fördern}

Der Einzug und die Förderung der Gehölze zum Häckselaggregat erfolgt durch die nun waagerecht ausgerichteten Förderelemente sehr zuverlässig. So kann über die verstellbare Förderwalze der Anpressdruck auf die Gehölze entsprechend ihres Volumens eingestellt werden, um die notwendige Förderwirkung zu erreichen. Der Scharnierbandboden stellt sich als weitreichende Verbesserung heraus, da mit ihm auch kurzes Material in den Häckselspalt gefördert wird, was sonst zwischen Förderwalze und selbsteinziehender Häckselschnecke verbleiben und Materialstauungen auslösen kann. Die Verwendung der seitlichen Förderschnecken im Zuführbereich erweisen sich dagegen als ungeeignet. Sie erfüllen zwar ihren Zweck zur seitlichen Konzentration der Gehölze und erreichen dabei gleichzeitig einen Längstransport im Zuführschacht zum Häckselrotor, jedoch fallen sie oft durch eingeklemmtes Material aus. Das Problem ist auf das Verklemmen von dünnen Ästen zwischen den Windungen und dem Schneckentrog lokalisiert. Trotz Versuchen die Zuverlässigkeit durch Änderungen der Troggeometrie und der Umkehr der Drehrichtung zu steigern, können diese häufig auftretenden Störungen nicht verhindert werden. Wie WIENEKE (1957) in seinen Arbeiten darlegt, ist die Wickelneigung von Schnecken gering. Dieses kann bei den Einsätzen 
sowohl bei der Aufnahme von abgestorbenem langem Gras als auch in Hundsrose bestätigt werden. Aufgrund der Störanfälligkeit durch das Verklemmen müssen sie dennoch im Verlauf der Versuchsarbeiten gegen glatte Trichterwände ausgetauscht werden (Abb. 64), da weitere aktiv arbeitende Fördersysteme, wie Förderbänder oder Förderzinken, baulich in diesem Bereich nicht $z u$ integrieren sind. Entsprechend wird auch die Förderwalze angepasst.

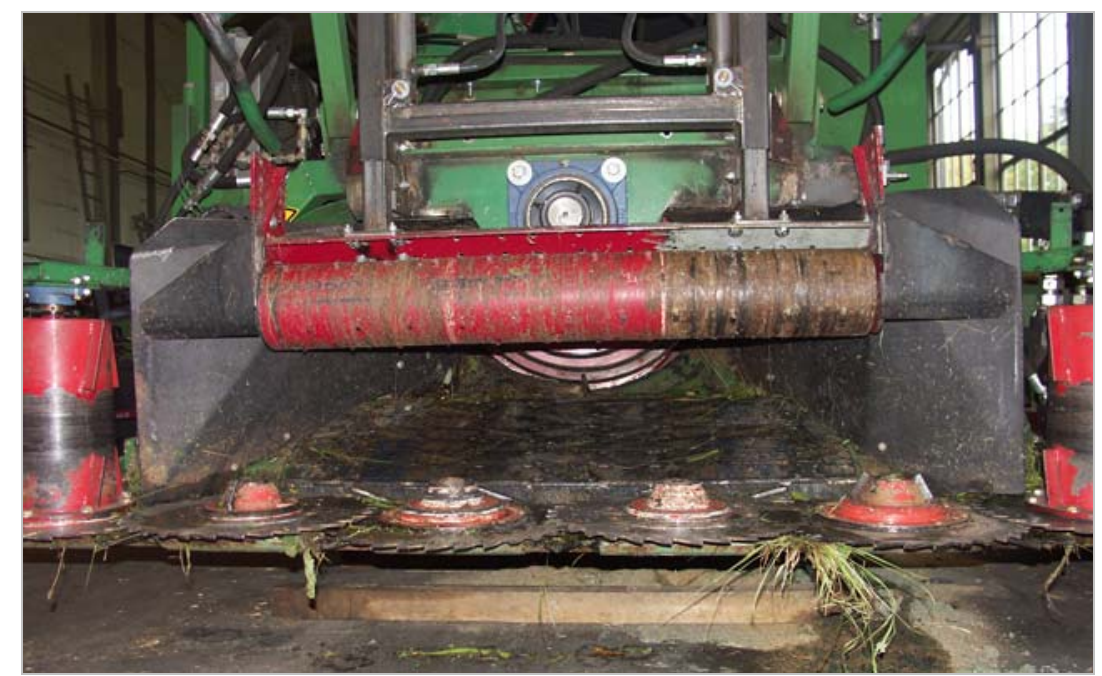

Abb. 64: Gehölzmähhäcksler 06 nach Austausch der Förderschnecken gegen glatte Seitenwände zur Materialkonzentration zum Häckselaggregat

Der Umbau stellt sich in den anschließenden Einsätzen als wirksame Maßnahme heraus, da die Materialkonzentration auch ohne bewegte Elemente auskommt und eine ausreichende Förderung zum Häckselaggregat gewährleistet ist. Lediglich kurze und leicht biegsame Gehölztriebe stellen sich zeitweilig im Förderbereich quer und können Materialstauungen verursachen. Diese Gehölze werden oft quer zur Förderrichtung von der Häckselschnecke eingezogen. Dabei erfolgt die Zerkleinerung aufgrund der nicht idealen Zuführung nur ungenügend, was durch einen hohen Anteil an Überlängen in dem dabei produzierten Material auffällt. Die Förderschnecken scheinen auch eine Wirkung auf die Ausrichtung der Gehölzachsen parallel zur Häckselrotorachse zu haben, da derartige Materialstauungen und die offensichtlich negative Auswirkung auf die Zerkleinerungsarbeit vorher nicht zu beobachten waren. 


\subsubsection{Zerkleinern}

Die Zerkleinerung wird sehr zuverlässig von dem Schneckenhäckslerrotor geleistet und der Selbsteinzug zeigt analog der Erfahrungen zum GMH 05 seine unterstützende Wirkung bei der Zuführung der Gehölze.

Die zusätzlichen Anschläge am Rotorumfang haben sich als wirksames Mittel herausgestellt, um eine Verteilung des Häckselvorganges auf den gesamten Rotorumfang zu erreichen. Das Einklemmen der Triebe bei der Zerkleinerung an den zusätzlichen Anschlägen erfolgt sicher und Zuführprobleme, die aufgrund der Zuführrichtung aus dem Förderbereich resultieren können, können nicht beobachtet werden. Sie sind somit ein zuverlässiges Mittel um den Häckselprozess um den Rotorumfang zu verteilen.

Allgemein treten Blockaden des Häckselrotors durch eine zu hohe Materialzufuhr selten auf, jedoch ist es beim GMH 06 aufgrund des im Verhältnis zum GMH 05 größeren Zuführschachts schwieriger das Material in angemessener Dosierung zuzuführen. Der Einzug erlaubt es auch, nach einer Materialstauung verhältnismäßig große Mengen zuzuführen, die durch den Selbsteinzug und durch die groß dimensionierte Häckselrotorfläche auch vom Häckselrotor erfasst werden können. Schneidet dieser in das gestaute Material ein, ist die Selbsteinzugsleistung in der Regel so groß, dass es keine Möglichkeit gibt das Material zurückzuhalten und es letztlich verarbeitet werden muss. Übersteigt der Leistungsbedarf zur Verarbeitung der Materialmenge das Leistungsangebot des Schleppers, ist ein Abbruch des Häckselvorganges oder ein Absterben des Schleppers unumgänglich. Der Abbruch des Häckselvorganges kann beim GMH 05 durch die senkrecht stehenden Zuführaggregate vergleichsweise besser geleistet werden.

\subsubsection{4. Überladen}

Die Hackschnitzel können von dem neu gestalteten Auswurfkrümmer sehr zuverlässig verladen werden. Lediglich Blätter und geringe Mengen an Feinanteil können bei korrekter Ausrichtung durch die geringe Masse nicht vollständig in den Container verladen werden. Das Auftreten offensichtlich zu gering beschleunigter Hackschnitzel, wie beim Einsatz des GMH 05, kann 
nicht beobachtet werden. Der schon seit Beginn der Untersuchungen im Gebrauch befindliche Überladecontainer kann sich bei den Einsätzen wieder bewähren. Durch den seitlichen Versatz des Krümmers ist bei diesem Maschinenkonzept der Blick auf den Arbeitsbereich weit besser. Eine Beurteilung des Einzugs- und des Häckselbereiches kann auch beim GMH 06 ausreichend durch das Kamerasystem gewährleistet werden.

\subsubsection{Mobileinheit}

Insgesamt sind seitliche Schwingungen in der Aufhängung der Maschine sowie auch der Einfluss durch die Maschinenmasse auf den Schlepper weit geringer als beim GMH 05. Die Höhenführung kann beim GMH 06 durch die kürzere Baulänge und die geringere Auswirkung der Hebelwirkung zwischen Zugfahrzeug und Schneidbereich trotz auch hier nicht integrierter Hilfskomponenten besser realisiert werden. Da der Sägeblattbalken durch die im Schlepper aufgehängte Bauart in seiner quer zur Bearbeitungsrichtung liegenden Schnittebene in der Ausrichtung der Schlepperhinterachse folgt, kann bei der Überfahrung von Unebenheiten nicht immer über die gesamte Schnittbreite ein bodennaher Schnitt erreicht werden. Vor allem bei stärkeren Bodenkonturänderungen, welche in einer kürzeren Distanz als der Abstand von der führenden Schlepperhinterachse bis zur Schneidlinie auftreten, kann ein bodennaher Schnitt über die Mähbalkenbreite nicht immer erreicht werden, da eine Anpassung an die Bodenkontur nicht gleich möglich ist. So entstehen teilweise Triebstümpfe mit einer Höhe von $20 \mathrm{~cm}$, welche aus einer günstigeren Bearbeitungsrichtung nachgeschnitten werden müssen. Dieses fällt bei dem Einsatz des GMH 06 verstärkt auf der Versuchsfläche DB1 auf, auf der Bodenunebenheiten innerhalb kurzer Distanz vorliegen. Diese Fläche wurde mit dem GMH 05 nicht bearbeitet, weshalb dieses in vorherigen Einsätzen nicht festgestellt wurde.

\subsubsection{Hackschnitzelqualität}

Mit Hackschnitzeln, welche mit dem GMH 06 während der vollmechanisierten Entbuschung produziert werden, werden Siebversuche hinsichtlich ihrer Verwertbarkeit bzw. Handelseignung durchgeführt. 


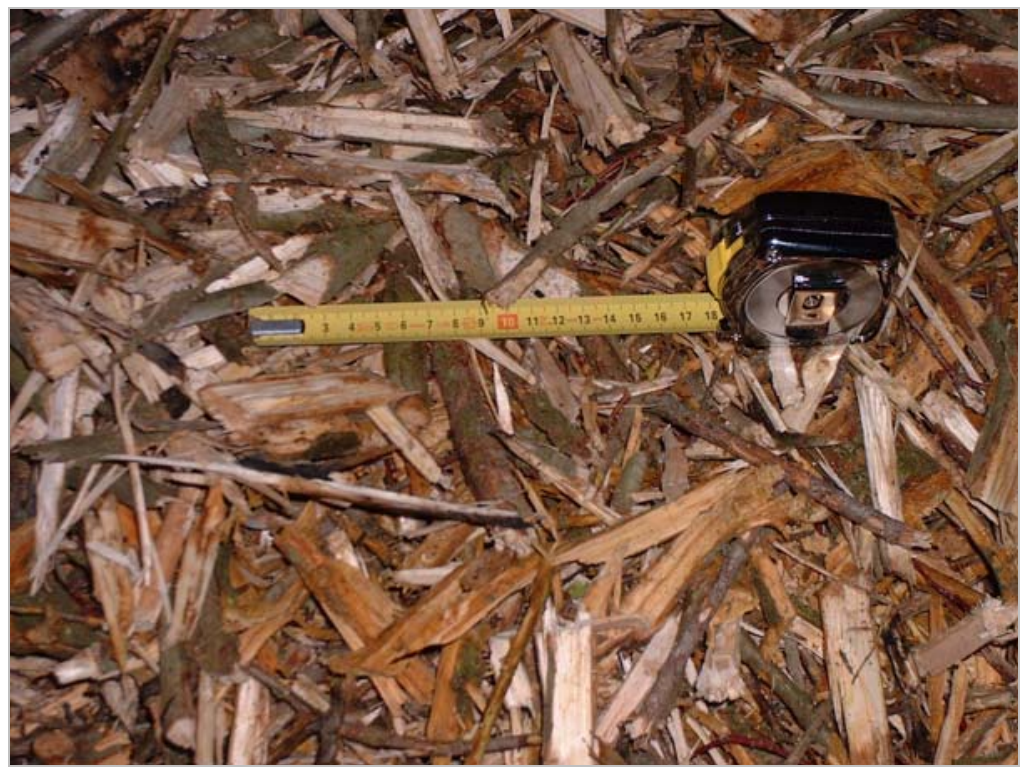

Abb. 65: In den Einsatzversuchen mit dem Gehölzmähhäckslers 06 produzierte Hackschnitzel

Abb. 66 zeigt das Siebergebnis einer Mischprobe aus den gesamten Einsatzversuchen. Die Hackschnitzel aus denen die Probe entnommen sind, sind nach dreimonatiger Lagerung augenscheinlich wenig verpilzt. Ein Anteil an gras- oder krautigen Bestandteilen kann in dieser Probe nicht festgestellt werden. Die Mischprobe weist einen Wassergehalt von $36 \%$ auf. Auffällig ist jedoch sofort der hohe Anteil an dünnen Ästen und verästelten Bestandteilen (siehe Abb. 65). Bei der Einzelbegutachtung einer Probe aus 312 Hackschnitzeln kann bei $46 \%$ keine Aufspleißung längs zur Faserrichtung festgestellt werden. Diese Triebabschnitte sind ausschließlich quer bzw. diagonal durchtrennt und ihr Durchmesser liegt dabei unterhalb von $2,5 \mathrm{~cm}$. Letztlich führt ein hoher Anteil an dünnen Ästen auch zu einer Ausprägung eines hohen Längen- Breiten Verhältnisses (siehe auch HASCHKE, 2000), was sich aufgrund des produzierten Grobhackgutes und des hier zu bearbeitenden Ausgangsmaterials nicht vermeiden lässt.

Die Hackschnitzel der Mischprobe lassen sich entsprechend der Siebergebnisse nicht in die gängige Hackschnitzelnorm CEN/TS 14961 einklassieren, da auch in der größten Klasse P100 der Anteil an Überlängen (in P100 >200 mm Hackgutgröße) zu hoch ist (1,9 statt <1 Massen- \%) (Abb. 
66). Da diese per Hand vermessen werden, kann dieses Ergebnis auch nicht auf eine Ungenauigkeit der Siebanalyse zurückgeführt werden.

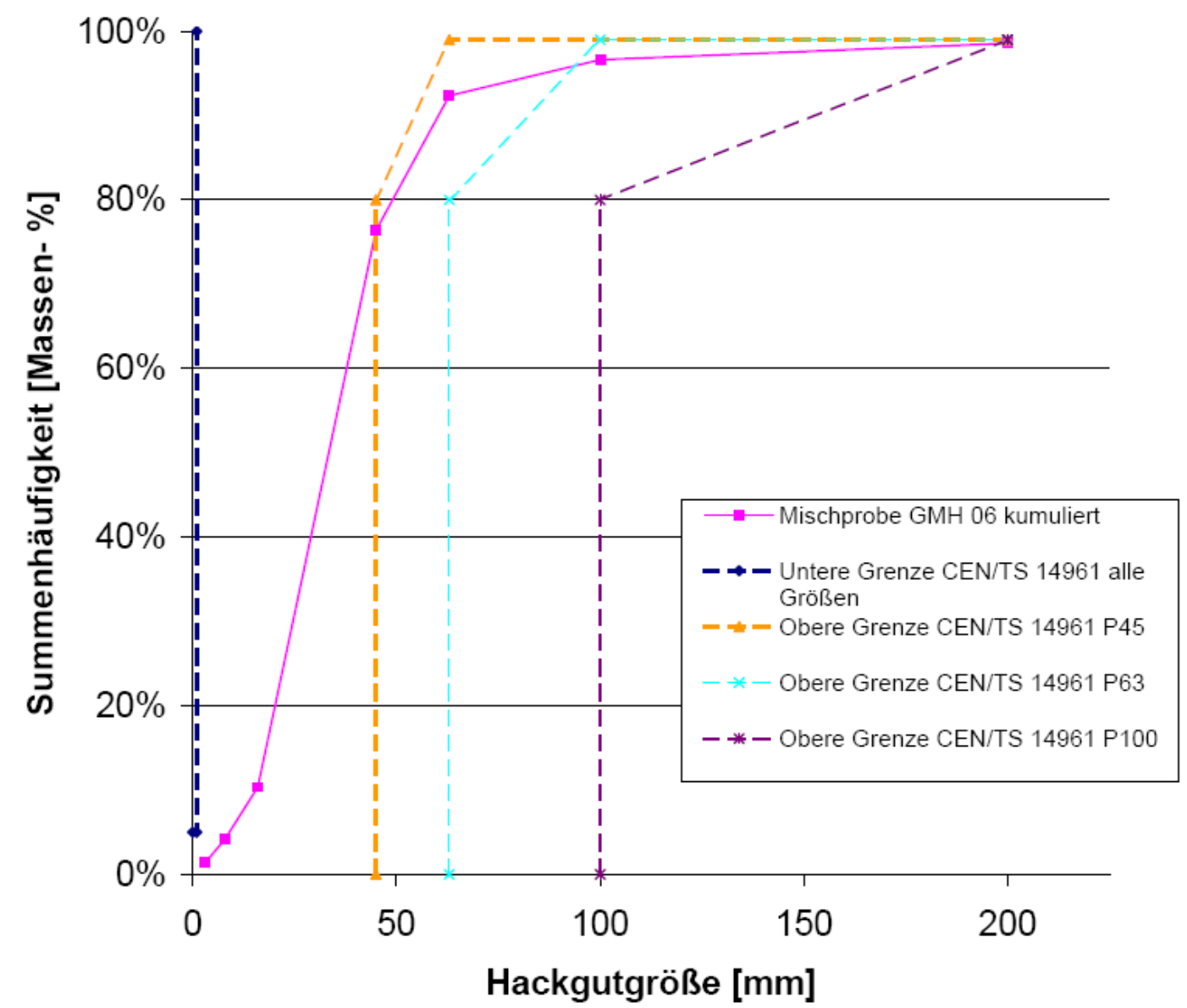

Abb. 66: Ergebnisse der Häufigkeit der Hackgutgrößen (kumuliert) aus einer Mischprobe von Entbuschungsmaterial; Material hergestellt bei Versuchseinsätzen mit Gehölzmähhäcksler 06 (Siebanalyse nach CEN/TS 15149-1)

In Tab. 8 sind die Ergebnisse der Siebanalyse des Hackschnitzelmaterials des Häckselversuches mit manueller Zuführung zum GMH 06 abgebildet. Sie zeigt die Anteile der Siebfraktionen der vier zerkleinerten Sortierungen nach bestimmten WHD- Klassen (1- $6 \mathrm{~cm}$; 6- $11 \mathrm{~cm})$ und unterschiedlicher Wuchsformen. Exemplarisch werden Esche für baum- und Weißdorn für buschartig eingesetzt. So werden vor allem bei dem buschartigen Weißdorn extrem hohe Anteile bei den Hackgutgrößen über 100 mm festgestellt. 
Der höchste Massenanteil lässt sich mit Abstand bei allen Sortierung im Größenbereich von 16- $45 \mathrm{~mm}$ finden. In diesem Bereich scheint offensichtlich die Hauptfraktion zu liegen, die der GMH 06 produziert.

Die einzelnen Massenanteile der vier Sortierungen werden in der Tabelle zudem miteinander verrechnet, indem die einzelnen Siebfraktionen miteinander addiert werden. Faktisch wird somit aus ihnen eine Mischprobe erstellt. Da die Weißdornsiebungen und vor allem die Weißdornsiebung mit geringem WHD bei den Überlängenanteilen hohe Werte aufweisen, werden die Weißdornanteile anschließend einzeln wieder herausgerechnet.

Tab. 8: Siebanalyse der Hackschnitzel (nach CEN/TS 15149-1) einer manuell zugeführten Zerkleinerung bestimmter WurzelhalsDurchmesserklassen mit dem Gehölzmähhäcksler 06; dargestellt als Massenanteile der Siebfraktionen in Prozent; WD = Weißdorn

\begin{tabular}{lcccccccc}
\hline $\begin{array}{l}\text { Größenbereich } \\
\text { [mm] }\end{array}$ & $\mathbf{2 0 0}$ & $\mathbf{2 0 0 - 1 0 0}$ & $\mathbf{1 0 0 - 6 3}$ & $\mathbf{6 3 - 4 5}$ & $\mathbf{4 5 - 1 6}$ & $\mathbf{1 6 - 8}$ & $\mathbf{8 - 3 , 1 5}$ & $<\mathbf{3 , 1 5}$ \\
\hline Esche (6-11cm) & $0,6 \%$ & $2,4 \%$ & $2,1 \%$ & $8,4 \%$ & $60,4 \%$ & $18,6 \%$ & $6,4 \%$ & $1,1 \%$ \\
Esche, (1- 6 cm) & $2,5 \%$ & $2,6 \%$ & $4,9 \%$ & $1,9 \%$ & $63,9 \%$ & $18,8 \%$ & $4,3 \%$ & $0,9 \%$ \\
WD, (6- 11cm) & $2,8 \%$ & $13,0 \%$ & $9,5 \%$ & $9,6 \%$ & $44,8 \%$ & $12,4 \%$ & $5,8 \%$ & $2,1 \%$ \\
WD, (1- 6 cm) & $6,1 \%$ & $16,5 \%$ & $12,5 \%$ & $11,0 \%$ & $38,4 \%$ & $9,6 \%$ & $4,3 \%$ & $1,7 \%$ \\
gesamt & $3,0 \%$ & $8,6 \%$ & $7,3 \%$ & $7,7 \%$ & $51,9 \%$ & $14,9 \%$ & $5,2 \%$ & $1,5 \%$ \\
$\begin{array}{l}\text { gesamt ohne WD } \\
\text { (1- 6 cm) }\end{array}$ & $2,0 \%$ & $6,0 \%$ & $5,5 \%$ & $6,6 \%$ & $56,4 \%$ & $16,6 \%$ & $5,5 \%$ & $1,4 \%$ \\
gesamt ohne & $1,6 \%$ & $2,5 \%$ & $3,5 \%$ & $5,1 \%$ & $62,2 \%$ & $18,7 \%$ & $5,3 \%$ & $1,0 \%$ \\
\hline WD & & & & & & & & \\
\hline
\end{tabular}

In Abb. 67 ist grafisch die Klassierung der vier WHD- und Wuchsformsortierungen nach den Ergebnissen der Siebfraktion aus Tab. 8 dargestellt. 


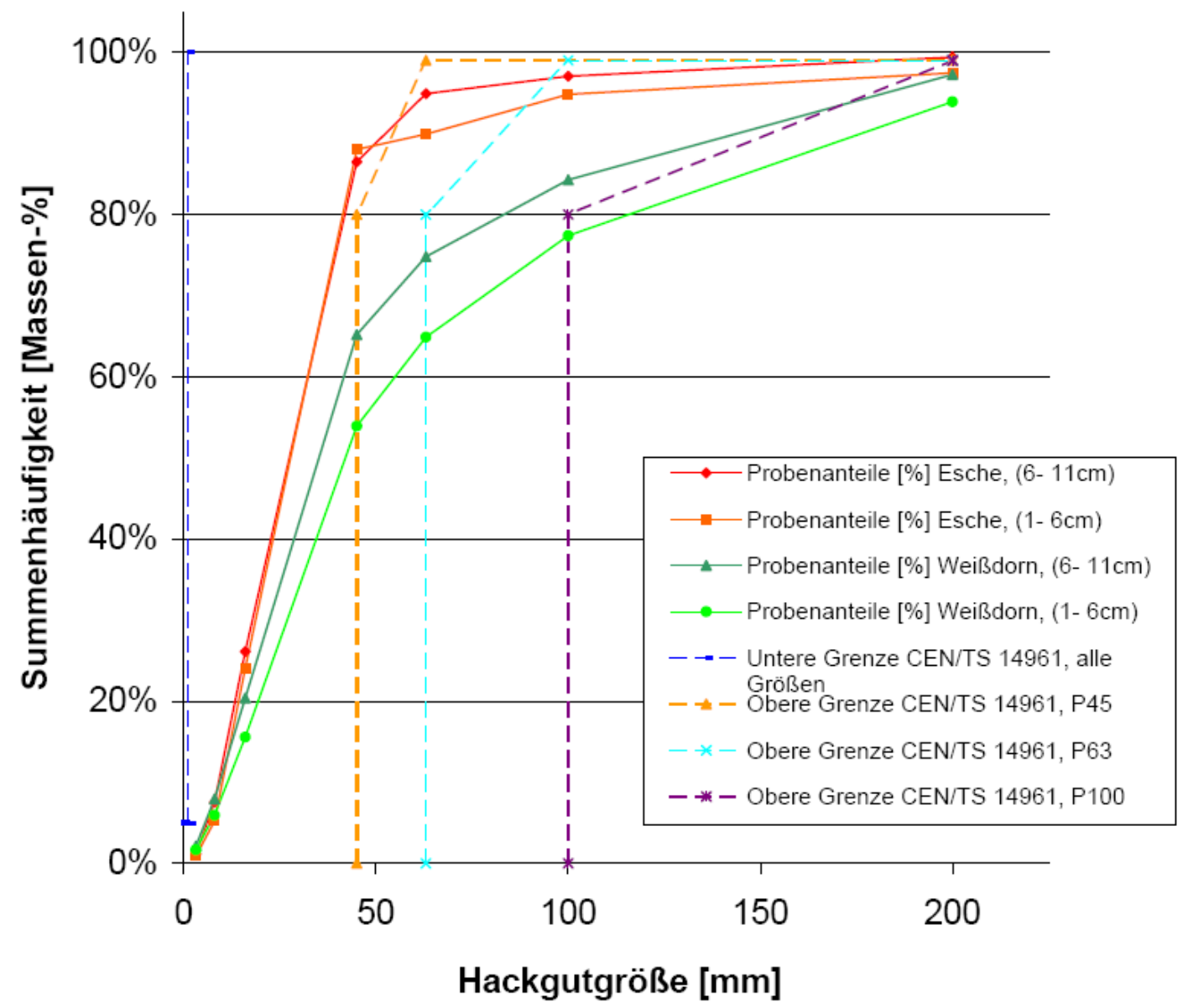

Abb. 67: Häufigkeit von Hackgutgrößen (dargestellt in Massenprozent kumuliert) in Hackgutproben, hergestellt mit dem Gehölzmähhäcksler 06 bei manueller Beschickung zur Zerkleinerung; eingetragen sind die obere und untere Anteilsgrenze (kumuliert) von Hackschnitzelgrößenklassierungen der gängigen Norm CEN/TS 14691 (Siebanalyse nach CEN/TS 15149-1)

Außer der Esche (6-11cm) erreicht keine Sortierung eine Klassierung nach CEN/TS 14961. Die Esche (6-11cm) kann nach der größten Hackschnitzelklasse P100 eingeordnet werden, da sie als einzige den geforderten Höchstanteil von 1 Massen- \% in der Siebfraktion $>200$ mm unterschreitet und im Bereich von 3,15-100 mm mit 95,9\% Massenanteil einen ausreichend hohen Wert erreicht, um den nach Norm geforderten Mindestanteil von 80 Massen- \% zu überschreiten.

Abb. 68 stellt die Ergebnisse der Verrechnung bzw. der einzelnen Mischungen der Sortierungen dar. Wie der Abbildung und auch Tab. $8 \mathrm{zu}$ entnehmen ist, ist es weder in der Gesamtmischung noch beim Eliminieren der stark mit Überlängen belasteten Weißdornsortierungen möglich, die 
Hackschnitzelproben in eine Größenklasse nach CEN/TS 14961 einordnen zu können. Selbst die Klassierung in der größten Klasse P100 ist aufgrund des zu hohen Überlängenanteils in der Fraktion >200 mm- Hackgutgröße nicht möglich, obwohl der Überlängenanteil ohne den Weißdorn annähernd halbiert werden kann.

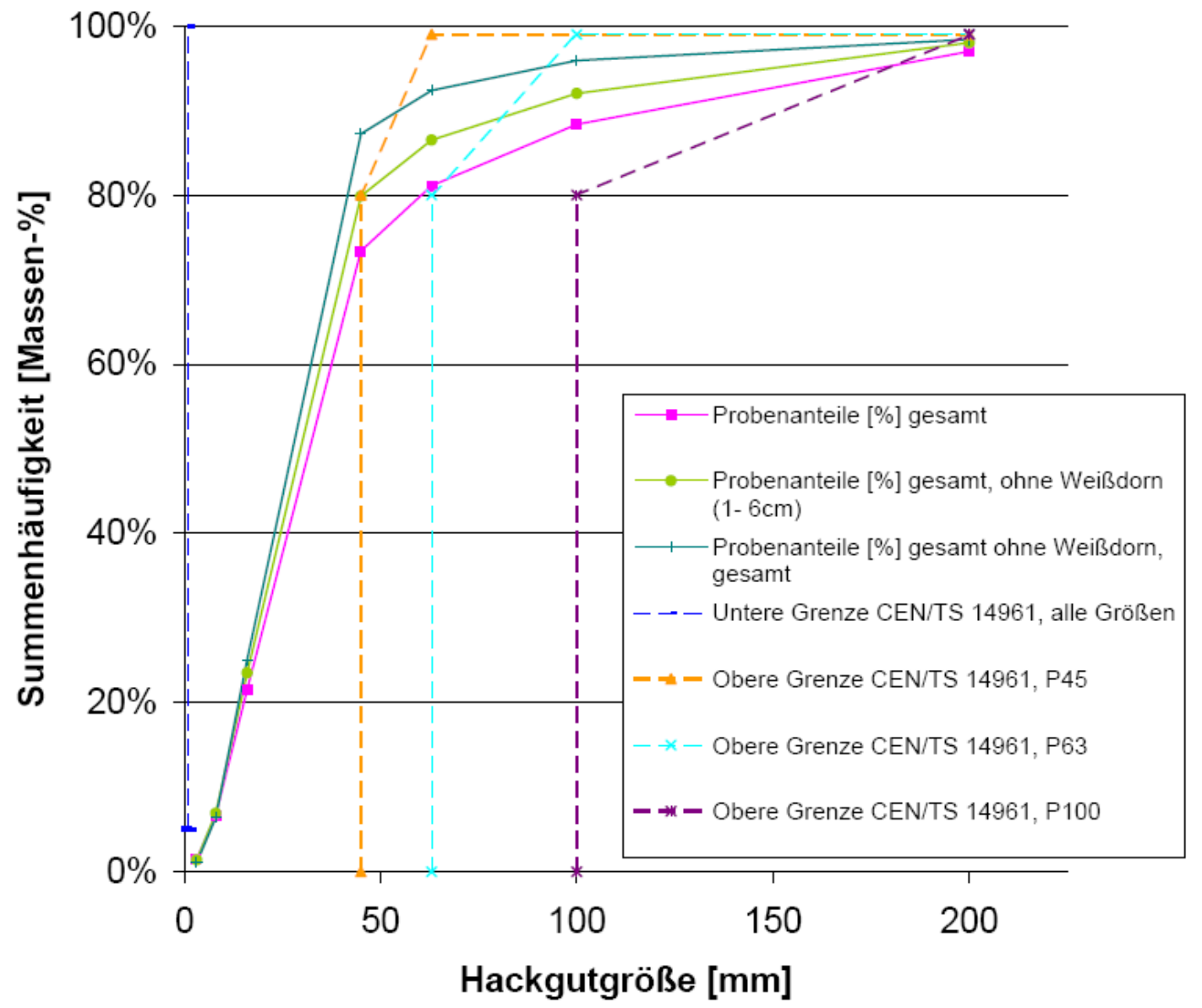

Abb. 68: Häufigkeit von Hackgutgrößen (dargestellt in Massenprozent kumuliert) in Hackgutproben verschiedener Sortierungsmischungen, hergestellt mit dem Gehölzmähhäcksler 06 bei manueller Beschickung bei der Zerkleinerung; eingetragen sind die obere und untere Anteilsgrenze (kumuliert) von Hackschnitzelgrößenklassierungen der gängigen Norm CEN/TS 14691 (Siebanalyse nach CEN/TS 15149-1)

Wie die Siebergebnisse zeigen, führt vornehmlich der Weißdorn zu einer sehr starken Zunahme an Überlängen im Häckselgut. Vor allem der Weißdorn mit geringem WHD erreicht hier hohe Werte. Dieses lässt den Schluss zu, dass das Schneckenhäcksleraggregat bei buschartigem Material eher zu Überlängen neigt. Dieses gilt jedoch auch für andere 
Hackerbauarten (BRUSCHE, 1983) und wird mit den stärker von der Stammachse abgewinkelten Ästen begründet, die vermehrt parallel zur Schnittebene der Messer zugeführt werden. Da die buschartigen und auch die dünnen baumartigen Triebe in dem Anwendungsgebiet des GMH 06 prinzipiell zu erwarten sind, bedeutet dieses, dass die Aufarbeitungsqualität des Hackaggregates verbessert werden muss, um klassierbare Hackschnitzel auch bei diesen Durchmessern und Wuchsformen produzieren zu können.

\subsubsection{Evaluation des GMH 06}

Hinsichtlich der Bodenkonturanpassung und der Höhenführung wird das Maschinenkonzept eine Verbesserung erfahren müssen. Im Versuchseinsatz ist die anspruchsvolle Schnitthöheneinstellung vom Schlepper aus zu tolerieren, für einen längerfristigen Einsatz wird sie jedoch zu aufwendig sein. Die technischen Möglichkeiten dazu werden schon beim GMH 05 (Kap. 5.4.2.4) angesprochen. Prinzipiell ist der Anspruch an einen bodennahen Schnitt jedoch erfüllbar.

Das Schneidvermögen und damit auch letztlich der größte bearbeitbare Durchmesser des Maschinenkonzeptes GMH 06 zeichnet sich auch hier im Bereich des WHD von $12 \mathrm{~cm}$ aufgrund des Getriebekastens ab. Ebenso kann eine Verringerung des Schneidvermögens in Abhängigkeit von der Schnittfläche festgestellt werden, die auf einem vermutlich $\mathrm{zu}$ hohen Leistungsbedarf beruht. Das Abtrennen der Gehölze kann somit technisch in dem geforderten Bereich der Anforderungsliste erfüllt werden, ist jedoch auch auf eine bestimmte Bestandesdichte beschränkt, da nur eine gewisse Schnittfläche gleichzeitig bearbeitet werden kann.

Bei der Materialaufnahme und der Materialzufuhr werden beim Einsatz des GMH 06 ähnlich des GMH 05 Grenzen der Bearbeitbarkeit deutlich. Eine sichere Aufnahme und Übergabe des Materials an die Zerkleinerungsaggregate kann prinzipiell nach diesem Arbeitsprinzip geleistet werden. Der Vorgang der waagerechten Ausrichtung der Triebe im Mähvorgang wird jedoch mit zunehmender Vegetationsdichte und mit 
zunehmender Biegesteifigkeit der Gehölze erschwert, bis ein Mähvorgang nicht mehr möglich ist. Auch beim Vorspannen der Triebe wird somit der Einfluss der Faktoren Wuchsform und Bestandesdichte auf das Entbuschungsvermögen des GMH deutlich, da es den bearbeitbaren WHD limitiert. Aus den durchgeführten Praxiseinsätzen können zum Einfluss dieser Faktoren auf das Entbuschungsvermögen nur die Tendenzen in Abhängigkeit von den Vegetationsparametern dargestellt werden. Abb. 69 stellt dazu das Entbuschungsvermögen mit dem GMH 06 als Breite der Pfeile dar, welches je nach Ausprägung der Vegetationsparameter zu- oder abnimmt.

\begin{tabular}{|c|c|c|}
\hline Wuchsform & $\begin{array}{c}\text { Bestandes- } \\
\text { dichte }\end{array}$ & $\begin{array}{c}\text { WHD- } \\
\text { Durchmesser }\end{array}$ \\
\hline baumartig & freistehend & dünn \\
\hline & & \\
buschartig & sehr dicht & sehr dick \\
\hline
\end{tabular}

Abb. 69: Tendenzen der Änderung des Entbuschungsvermögens des GMH 06 bei der Entbuschung von Offenflächen in Abhängigkeit von der Ausprägung von Vegetationsparametern; Pfeilbreite gleich Höhe des Entbuschungsvermögens

Abb. 69 zeigt, dass sich das über den WHD definierte Entbuschungsvermögen des GMH zumindest aufgrund der Bestandesdichte und der Wuchsform vermindert und sich in weiterer Konsequenz eine Verringerung des bisher ausschließlich über den WHD definierten Einsatzbereiches ergibt. Die maximal technische Grenze des Entbuschungsvermögens von $12 \mathrm{~cm}$ WHD, welche baulich durch den Sägeblattbalken vorgegeben ist, ist demnach eine Maximalgröße des 
Maschinenkonzeptes, die bei günstigen Bestandesdichten und Wuchsformen technisch geleistet werden kann. Zu einer Beurteilung der Bearbeitbarkeit einer verbuschten Offenfläche müssen demnach die Faktoren Bestandesdichte und Wuchsform auch mit einfließen. Der Versuch zur Quantifizierung dieser offensichtlich vielseitigen Abhängigkeiten kann im Rahmen dieses Vorhabens aufgrund der dazu umfangreich notwendigen Untersuchungen jedoch nicht mehr durchgeführt werden.

Der Einsatz des Schneckenhäcksleraggregates zur Zerkleinerung stellt sich vor allem durch den Selbsteinzug zur Unterstützung des Einzugsprozesses als vorteilhaft dar. Die Aufarbeitung des Gehölzmaterials im Rahmen der Entbuschungsmaßnahmen ist ohne Weiteres möglich. Der bisher bestehende Aufwand zur Lösung von Blockaden bei diesem System muss jedoch technisch noch implementiert werden. Auf die Lösungsvorschläge dazu wurde schon in vorigen Kapiteln zum GMH 05 eingegangen und eine Umsetzung erscheint an dem vorhandenen Maschinenkonzept ebenfalls durchführbar.

Die Aufarbeitungsqualität der Hackschnitzel ist weniger zufriedenstellend und lässt hinsichtlich der Anforderung an eine Verwertbarkeit zumindest keine Klassierbarkeit in die bestehende Brennstoffnorm zu. Auch wenn dieses Ziel in diesem Maschinenkonzept knapp verfehlt wird, so kann zumindest ein vorkonfektioniertes Material erstellt werden, was bei einer Aufwertung z.B. durch eine Siebung einer Verwertung überführt werden kann. Der erhöhte Anteil an Überlängen bei Reisig im Gegensatz zu Stammholz wird auch von BRUSCHE (1983) und von FIRUS \& BELTER (1995b) bei Untersuchungen zur Zerkleinerung beschrieben. Sie alle führen dieses auf die ungenügende Ausrichtung der Gehölzachsen bzw. der Astachsen zu den Schneiden im Zuführungsprozess zurück. Dieses ist bei Reisig durch die vielen von der Stammachse abgewinkelten Äste schwieriger als bei einem Stamm und wird sich bei buschartigen Gehölzen durch den ohnehin schon sparrigen Wuchs noch intensiver ausprägen. Die Siebergebnisse bestätigen diesen Zusammenhang durch die zunehmend hohen Anteile von Überlängen bei buschartigen und bei dünneren Gehölzen. Wie zu beobachten ist, stellen sich 
nach dem Entfernen der Zuführschnecken vor allem dünne Gehölze in dem verhältnismäßig breiten Einzug öfter quer und werden auch entsprechend dieser Ausrichtung von der Häckselschnecke eingezogen. Ein idealer Schneckenschnitt ist so nicht mehr möglich und das Auftreten von Überlängen die Folge. Dieser Zusammenhang erklärt auch die besseren Häckselergebnisse des GMH 05, der ein klassierbares Ergebnis der produzierten Hackschnitzel liefert. Die geteilten Einzugsbereiche der Häckselaggregate sind beim GMH 05 nur annähernd halb so breit, da diese nur das Material der halben Maschinenbreite aufnehmen müssen. Aufgrund der geringeren Breite müssen dort auch kürzere Triebe mit ihrer Triebachse parallel auf die Häckselschnecke ausgerichtet sein, um hindurchgefördert werden zu können. Dieses und das stärkere Komprimieren innerhalb des engeren Förderweges begünstigen ihre ideale Zerkleinerung im Häckselaggregat und minimieren das Aufkommen von Überlängen. Bei einer Weiterentwicklung des Häckselaggregates wird dieser Zusammenhang sicherlich von Bedeutung sein. Um die direkte Verwertbarkeit sicherzustellen, ist eine Optimierung des Häckselaggregates im Bezug auf die Hackgutqualität bei geringen WHD unumgänglich.

Grundsätzlich kann bei einer Überarbeitung des Zerkleinerungssystems auch der Wechsel auf ein anderes Zerkleinerungsprinzip wie ein Trommelhackeroder Scheibenradhackersystem in Erwägung gezogen werden. Diese werden jedoch zum Vollzug eines idealen Schnittes nicht mindere Anforderungen an die Materialausrichtung der Zuführung stellen, damit Überlängen wirkungsvoll vermieden werden können (MAIER, 1983; BRUSCHE, 1983). Des Weiteren ist ein vergleichbarer Selbsteinzugseffekt nicht möglich. Die Ergebnisse von Nurmi (1986) zur Holzzerkleinerung mit unterschiedlichen Hackschnecken ermutigen hier eher zur Verwendung eines geringeren Windungsabstandes und zur Produktion einer insgesamt geringeren Hackschnitzelgröße, um einer Normerfüllung entgegen $z u$ kommen. Dabei wird vermutlich eine geringere Lagerstabilität des Materials berücksichtigt werden müssen (SCHOLZ \& IDLER, 2005) und die Verwendung von mehrgängigen Häckselschnecken, um eine ausreichende Einzugsgeschwindigkeit zu erhalten. 
Die Überladesicherheit kann mit dem neu gestalteten Überladekrümmer sicher und ohne nennenswerte Verluste gewährleistet werden. Inwieweit die nicht verladenen Blattanteile bei Arbeiten in der Vegetationsphase und der Feinanteil (vornehmlich Rinde) zu bewerten sind, muss sicherlich nach einer Quantifizierung im Einsatzfall sowohl naturschutzfachlich als auch technisch abgewogen werden. Einerseits besitzen diese Anteile prinzipiell höhere Nährstoffgehalte (WITTKOPF ET AL., 2003), weshalb eine Abfuhr zur Aushagerung sicherlich gewünscht ist, andererseits verschlechtern sie zusätzlich die Qualität hinsichtlich einer Verwertung (BAUER, 2005).

Der zum Flächentransport eingesetzte Überladecontainer kann aufgrund der Gesamterfahrungen bei der Entwicklung als sehr gute Lösung zur Bergung des Gehölzmaterials in diesem Konzept bewertet werden. Er lässt sich optimal in das Maschinenkonzept integrieren.

Die Bedienung und Beurteilbarkeit der Arbeitszustände an der Maschine sind soweit verbessert worden, so dass eine vollmechanisierte Entbuschung im Mähverfahren von nur einem Bediener zu leisten ist. Die Höhenführung des Schnittes über die Schlepper- Drei- Punkt- Hydraulik und die Führung der Schnittebene durch die Schlepperhinterachse können jedoch im Praxisbetrieb keine Dauerlösung darstellen. In sehr unebenem Gelände ist nur schwer eine ausreichende Konturanpassung der Schnittebene an die Bodenoberfläche zu erreichen, um den bodennahen Schnitt umfangreich sicherzustellen. Zur Gewährleistung sind Führungssysteme in ihrer Funktion denkbar, wie sie z.B. auch aus der Mähernte von Feldfrüchten bei Mähdreschern etc. eingesetzt werden. Ihre technische Umsetzung wird aus den schon beim GMH 05 beschriebenen Umständen weitaus anspruchsvoller sein, sie scheint aber unabdingbar.

Da der Einsatz des GMH 06 zum Ende der Projektphase erfolgt, können keine tiefgreifenderen Untersuchungen zu den Wirkungen seines Einsatzes mehr durchgeführt werden. Während und direkt nach den Einsätzen sind visuell keine bedenkenswerten Einflüsse erkennbar. Da das Arbeitsprinzip hinsichtlich seiner Umweltwirkungen mit dem des GMH 05 vergleichbar ist, ist deren Übertragung auf dieses Maschinenkonzept vorerst sicherlich zu 
rechtfertigen. Dieses muss aber durch weitere Untersuchungen abgesichert werden. Aus diesem Grund soll die Erfüllung dieser Punkte in der zusammenfassenden Darstellung der Anforderungslisten in Tab. 9 und Tab. 10 auch bewusst als „bisher nicht abschätzbar" eingestuft werden.

Tab. 9: Erfüllung bzw. potenzielle Erfüllbarkeit naturschutzfachlicher Anforderungen anhand der Ergebnisse zum GMH 06; v : erfüllt; $x$ : nicht erfüllt (+/- : positive/negative Tendenz zur Erfüllbarkeit); ? : bisher nicht sicher abschätzbar

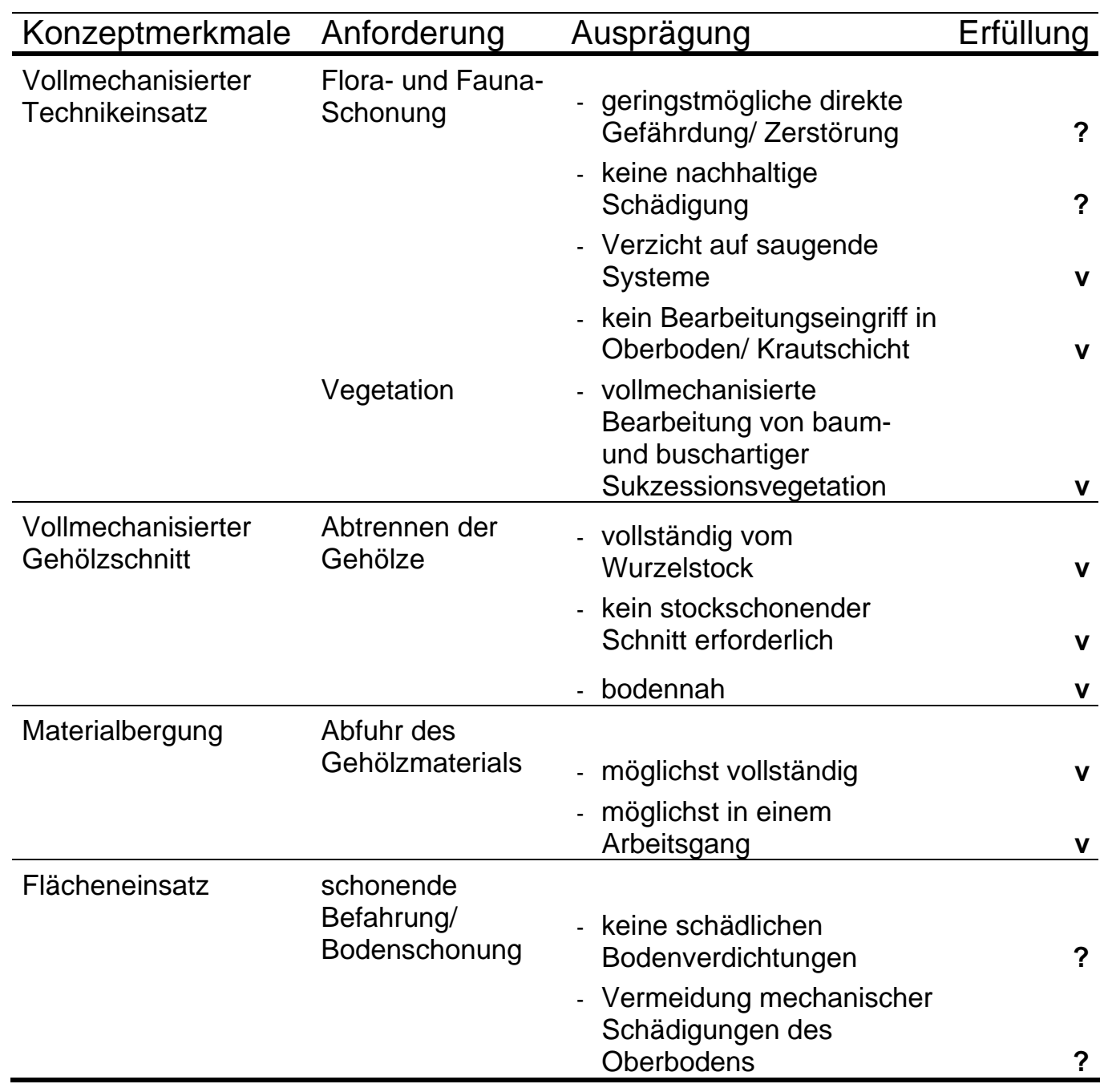


Tab. 10: Erfüllung bzw. potenzielle Erfüllbarkeit technischer und technologischer Anforderungen anhand der Ergebnisse zum GMH 06; v : erfüllt; $x$ : nicht erfüllt (+/- : positive/negative Tendenz zur Erfüllbarkeit); ? : bisher nicht sicher abschätzbar

\begin{tabular}{|c|c|c|c|}
\hline Konzeptmerkmale & Anforderung & Ausprägung & \\
\hline \multirow[t]{2}{*}{ Technikausrichtung } & $\begin{array}{l}\text { Einbindung in } \\
\text { bestehende } \\
\text { Technikstrukturen }\end{array}$ & $\begin{array}{l}\text { - Nutzbarkeit allgemein verfügbarer } \\
\text { Trägerfahrzeuge }\end{array}$ & $\mathbf{v}$ \\
\hline & & - Nutzbarkeit bestehender Logistikkonzepte & $?$ \\
\hline \multirow[t]{2}{*}{ Technisierung } & Vollmechanisierung & - nur Bediener & $\mathbf{v}$ \\
\hline & & - keine händischen Arbeitsschritte & $\mathbf{v}$ \\
\hline \multirow[t]{4}{*}{ Materialbergung } & Abfuhr des & & \\
\hline & Gehölzmaterials & & $\mathbf{v}$ \\
\hline & & $\begin{array}{l}\text { - Überladung oder Konzentration des } \\
\text { Materials für den Abtransport }\end{array}$ & $\mathbf{v}$ \\
\hline & Aufarbeitung & - möglichst in verwertbarer Form & $\mathbf{v}$ \\
\hline \multirow[t]{3}{*}{ Flächeneinsatz } & flächige Bearbeitung & - Überfahrbarkeit der Fläche & v \\
\hline & $\begin{array}{l}\text { Manövrierbarkeit/ } \\
\text { Beweglichkeit }\end{array}$ & $\begin{array}{l}\text { - Möglichkeit von Kurvenfahrt im } \\
\text { Arbeitsprozess }\end{array}$ & $\mathbf{v}$ \\
\hline & & - Rangieren auf engem Raum & $\mathbf{v}$ \\
\hline \multirow[t]{7}{*}{$\begin{array}{l}\text { Vollmechanisierter } \\
\text { Entbuschungsvorgang }\end{array}$} & $\begin{array}{l}\text { Schneiden der } \\
\text { Gehölze }\end{array}$ & $\begin{array}{l}\text { - vollständig bis mindestens } 10 \mathrm{~cm} \text { WHD } \\
\text { und möglichst darüber }\end{array}$ & $\mathbf{v}$ \\
\hline & & - Schnitthöhe beeinflussbar & $\mathbf{v}$ \\
\hline & $\begin{array}{l}\text { Aufnahme der } \\
\text { Gehölze }\end{array}$ & $\begin{array}{l}\text { - Aufnahme und sichere Übergabe an die } \\
\text { Aufbereitung }\end{array}$ & $\mathbf{v}$ \\
\hline & $\begin{array}{l}\text { Aufbereitung der } \\
\text { Gehölze }\end{array}$ & - energieeffizient & ? \\
\hline & & - transportwürdige Aufarbeitung & $\mathbf{v}$ \\
\hline & & - logistisch vorteilhaft & $\mathbf{v}$ \\
\hline & & - möglichst verwertbares Endprodukt & $\mathbf{v}$ \\
\hline \multirow[t]{2}{*}{ Bedienung } & Ferneinstellung & $\begin{array}{l}\text { - alle Betriebsfunktionen vom Bedienplatz } \\
\text { einstellbar }\end{array}$ & $\mathbf{v}$ \\
\hline & Übersichtlichkeit & $\begin{array}{l}\text { - alle Betriebszustände vom Bedienplatz } \\
\text { ausreichend beurteilbar }\end{array}$ & $\mathbf{v}$ \\
\hline Sicherheit & Arbeitssicherheit & $\begin{array}{l}\text { - größtmöglicher Schutz des } \\
\text { Bedienpersonals }\end{array}$ & $\mathbf{v}$ \\
\hline \multirow[t]{2}{*}{ Haltbarkeit } & Verschleiß & - möglichst hohe Standzeit & $\mathbf{x}$ \\
\hline & Festigkeit & - ausreichende Stabilität der Komponenten & $\mathbf{x}$ \\
\hline
\end{tabular}




\subsection{Einordnung des Maschinenkonzeptes GMH 06 in die}

\section{Verfahren zur vollmechanisierten Beseitigung von}

\section{Verbuschungen auf Offenflächen}

In diesem Kapitel soll das Maschinenkonzept des GMH 06 als Verfahren zur Entbuschung von Offenflächen bei jetzigen Stand der Entwicklung und Erkenntnisse betrachtet werden. Der GMH 06 kann dabei ein neues Verfahren zur Entbuschung von Offenflächen darstellen, welches innerhalb der vorhanden Verfahren anhand seiner naturschutzfachlichen sowie auch technischen Möglichkeiten sowie auch der Wirkungen des Einsatzes gemessen werden wird.

Der GMH ist aufgrund des Mähkonzeptes eines der wenigen verfügbaren vollmechanisierten Verfahren, dass eine gleichzeitige Materialbergung in einem Arbeitsschritt erlaubt. Letztlich aber das einzige Verfahren, was eine Entbuschung ohne einen Bodeneingriff in einem Arbeitsgang leisten kann. Dieses stellt ein Alleinstellungsmerkmal des Verfahrens dar und lässt es verfahrenstechnisch sowie auch naturschutzfachlich vorzüglicher bei der Auswahl werden. Dabei kommt ihm entgegen, dass es in Zeiten eines hohen Interesses an energetisch verwertbarer Biomasse ein zumindest potenziell verwertbares Holzhäckselgut herstellen kann. Bei einer Vermarktung kann dieses letztlich auch eine Möglichkeit zur Kostenreduktion von Entbuschungsmaßnahmen sein (CREMER ET AL., 2007).

Inwieweit sich der Überlängenanteil auf die letztendliche Marktfähigkeit niederschlagen würde, bleibt offen. Jedoch berichtet Bauer (2005) dazu, dass die im Vergleich zu Waldhackschnitzeln minderwertigeren Landschaftspflegehackschnitzel bei gewissen Preisabschlägen durchaus nachgefragt seien.

Aufgrund der Schlepperbasierung des Verfahrens besitzt der GMH, wenn er auch selbst eine Spezialmaschine darstellt, nicht die Verfahrensspezialisierung, wie es z.B. bei der Mäh- Laderaupe zu finden ist. Die Schlepperbasierung schränkt inn in der Anwendbarkeit z.B. hinsichtlich Hangtauglichkeit, Befahrbarkeit etc. auf die technischen Möglichkeiten dieser 
Fahrzeuge ein, jedoch besitzt die Verwendbarkeit dieser Basisfahrzeuge auch einen Vorteil in der Verfahrensverfügbarkeit. Schlepper sind flächendeckend vorhanden und Land- sowie Forstwirte führen heute schon einen großer Teil der Pflegeaufgaben mit ihren Maschinen aus (JEDICKE, 1996). Dieses kann einerseits einen Akzeptanzvorteil bedeuten und andererseits ist es bezüglich des Transportes leichter möglich, den GMH als Anbaumaschine überregional zur Verfügung zu stellen.

Trotz aller Vorteile, die der GMH aufweist, wird er jedoch nur ein zusätzliches Verfahren zu den bestehenden darstellen können. Dieses liegt einerseits in den genannten technischen Einschränkungen der Basisfahrzeuge begründet. Pflegeflächen sind Z.B. nicht immer von Schleppern befahrbar (JEDICKE, 1996). Andererseits kann auch der GMH technisch nicht den gesamten Anwendungsbereich der Entbuschung hinsichtlich der zu bearbeitenden Gehölzvegetation abdecken. Aus diesem Grund werden andere Verfahren weiterhin zur Auswahl kommen müssen, nachdem ihr Einsatz nach naturschutzfachlich oder auch technisch Möglichkeiten bzw. Restriktionen abgewogen wurde.

Der GMH wird demnach nur einen Teilbereich bearbeiten können und neben den anderen Verfahren bestehen können. Dafür sind die Kriterien der Technikauswahl in Hinsicht der Flächen- und Vegetationsbedingungen sowie auch der naturschutzfachlichen Belange zu umfangreich (vergl. z.B. Kap. 3.1.3; INSTITUT FÜR AGRARTECHNIK BORNIM ; VDI- GESELLSCHAFT FÜR AgraRtechnik, 1994; Prochnow \& Schlauderer, 2002; Jedicke, 1996; BAUER ET AL., 2004).

Wie in den vorherigen Kapiteln beschrieben, ist es zum derzeitigen Stand der Erkenntnisse nur möglich, eine quantitative Aussage über den technisch möglichen Maximalbereich der Anwendung des $\mathrm{GMH}$ unter günstigen Vegetationsbedingungen widerzugeben. Dieser liegt bei $12 \mathrm{~cm}$ WHD und wird sich unter ungünstigeren Vegetationsbedingungen erniedrigen. Dieser Zusammenhang lässt vermuten, dass sich die Einsetzbarkeit der Vergleichsverfahren hinsichtlich ihres Anwendungsbereiches ebenfalls über ihren in dieser Arbeit ausschließlich nach dem WHD definierten 
Arbeitsbereich verschieben werden. Bei den Vergleichsverfahren handelt es sich bei den Literaturangaben jedoch unterhalb des ermittelten Defizitbereiches an verfügbarer Entbuschungstechnik (Kap. 5.1) um Maximal- und oberhalb des Bereiches um Minimalwerte des Entbuschungsvermögens. Der ermittelte Defizitbereich würde sich entsprechend einer Verschiebung der Vergleichsverfahrenswerte durch den Einflusses der Vegetationsbedingungen nicht verkleinern sondern eher vergrößern. Dadurch würde einem dort angesiedelten Verfahren, wie es der $\mathrm{GMH}$ ist, eine höhere Bedeutung zukommen.

Dass sich bestehende Verfahren letztlich gut mit dem GMH kombinieren lassen, kann anhand der weiteren Entwicklung der Versuchsflächen beispielhaft dargestellt werden. So berichtet der Bewirtschafter der Versuchsfläche DB2 von der nun möglichen Pflegbarkeit der Fläche mit dem landwirtschaftlichen Mulcher (BERTSCH, 2008). Sie ist in Abb. 70 dargestellt. Des Weiteren konnte in den floristischen und faunistischen Begleituntersuchungen festgestellt werden, dass die nachfolgende Beweidung der freigestellten Flächenteile auf DB3 den jungen Wiederaustrieb der Gehölze effektiv zurückdrängen kann und eine Zunahme typischer Zeigerarten des Offenlandes festzustellen ist (MEINEKE, 2006).

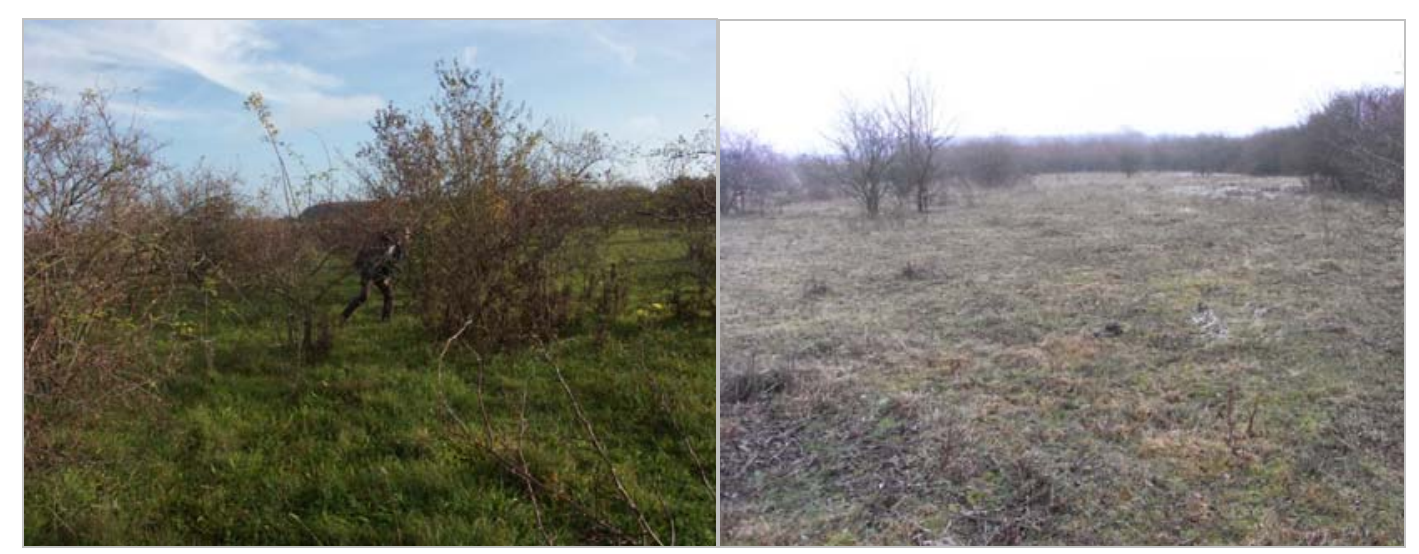

Abb. 70: Blick auf die Versuchsfläche Drakenberg 2 (DB2) vor (links, spätes Frühjahr 2007) und nach der Entbuschung mit dem Gehölzmähhäcksler 06 (Winter 2007) 


\section{Forschungsbedarf}

Grundsätzlich stellt die Entwicklung dieser Arbeit bisher nur das Konzept und eine Prototypmaschine zur vollmechanisierten Entbuschung von Offenflächen. Da sich diese noch in einer frühen Entwicklungsphase befinden, zeigen sowohl die Maschine selbst als auch deren Anwendung Möglichkeiten und Notwendigkeiten zu weiterem Forschungsbedarf auf, um das Verfahren des GMH letztlich technisch sowie auch naturschutzfachlich in Anwendung und Wirkung weiter abzusichern und zu verbessern.

Das Maschinenkonzept und seine Komponenten zeigen zwar ihre Funktion, aber auch an gewissen Stellen Optimierungsbedarf oder sie bedingen zur Verbesserung des Verfahrens weiterer Ausstattung.

Maschinenseitig ist hier sicherlich die Bodenkonturführung des Schnittes, die Reversierung des Häckselaggregates sowie der Optimierung der Aufarbeitungsqualität der Hackschnitzel zu nennen. Des Weiteren liegen bisher auch nur geringe Erkenntnisse über Standzeiten und konstruktiv bedingte Schwachpunkte des Maschinenaufbaus vor, da Langzeitaufnahmen des Maschineneinsatzes noch nicht durchgeführt werden konnten.

Die Quantifizierung von Einflussparametern der Vegetationsbedingungen und deren Wechselwirkung auf die Einsetzbarkeit sowie deren Auswirkungen auf Langlebigkeit und die technische Ausstattung konnte nicht im Mittelpunkt dieser Untersuchung stehen. Sie bietet ein weites Feld für weitere Forschungsfragen und ist erst als zweiter Schritt nach der umfangreichen Erstellung des geeigneten Maschinenkonzeptes möglich.

Letztlich ist die Anwendung des GMH bisher nicht ausreichend auf ihre naturschutzfachlichen Fragen hin untersucht worden. Die Ermittlung von Wirkungen auf Flora und Fauna bei verschiedensten jahreszeitlichen Anwendungen steht ebenso noch weitestgehend aus, wie umfangreichere Auswirkungen auf den Boden bei dieser Technikanwendung.

Schließlich werden sich bei der Beantwortung und der weiteren Entwicklung noch zusätzliche Fragen ergeben, die einer Untersuchung bedürfen oder würdig sind. 


\section{Fazit}

Mit dem GMH 06 konnte das vollmechanisiert arbeitende Maschinenkonzept eines schlepperbasierten Mähhäckslers erstellt werden, was die Funktionen von Schneiden, Aufnehmen, Fördern, Zerkleinern und Überladen von Gehölzen bei Entbuschungsmaßnahmen auf Offenflächen in einem Arbeitsgang ermöglicht. Die dabei hergestellten Holzhackschnitzel lassen sich beim Entbuschungsprozess im Bunkerverfahren bergen und von der Fläche abfahren. Dabei kann die Herstellung von Holzhackschnitzeln von diesem Maschinenkonzept ermöglicht werden. Eine energetische Verwertung des aufgearbeiteten Gehölzmaterials erscheint dabei möglich, eine Klassierbarkeit der Hackschnitzel in die gültige Brennstoffnorm lässt das Häckselaggregat bei der derzeitigen Aufarbeitungsgüte und bei dem zu erwartenden Ausgangsmaterial der Entbuschung in dem Einsatzbereich noch nicht ausreichend zu.

Zur Gewährleistung der Funktion des Maschinenkonzeptes erweist sich die Kombination der folgender Technikeigenschaften als zielführend:

- ein Schlepper als Basisfahrzeug

- ein Vorspannen der Gehölze vor dem Schneiden

- das Schneiden der Gehölze mit einem Sägeblattbalken,

- die Aufnahme von waagerecht und in Fahrtrichtung parallel zueinander ausgerichteter Gehölze,

- Zuführaggregate mit parallel zum Boden ausgerichteten Förderflächen,

- ein Schneckenhäcksleraggregat zur Zerkleinerung,

- ein Wurfgebläse zum Überladen

- ein Frontladercontainer am Basisfahrzeug zur Bergung des Materials

Der jetzige Entwicklungsstand des Maschinenkonzeptes ermöglicht, vollmechanisiert Gehölze mit einem WHD von $12 \mathrm{~cm}$ zu bearbeiten und dabei grundlegende naturschutzfachliche sowie technisch/technologische 
Anforderungen für den Anwendungsbereich zur Entbuschungen von Offenflächen mit bis zu 10 cm WHD zu erfüllen.

Dabei haben die Ergebnisse gezeigt, dass die Definition des Entbuschungsvermögens allein über den WHD nicht genügen wird, um den tatsächlich möglichen Anwendungsbereich des Maschinenkonzeptes hinreichend $\mathrm{zu}$ beschreiben. Bei den Faktoren Bestandesdichte und Wuchsform zeichnet sich ein nennenswerter Einfluss auf das Entbuschungsvermögen ab, deren Wechselwirkungen beim gegenwärtigem Erkenntnisstand zwar tendenziell aber nicht quantitativ eingeordnet werden kann.

Die naturschutzfachlichen Belange sind in der Entwicklung des Maschinenkonzeptes umfangreich berücksichtigt worden, inwieweit sie erfolgreich und im Hinblick auf langfristige Wirkungen umgesetzt werden können, muss Gegenstand weiterer Forschung sein. 


\section{Zusammenfassung}

Ein Kernproblem beim Erhalt und der Pflege landschaftlich und biologisch wertvoller Biotope der heutigen Kulturlandschaft stellt die Entbuschung von Offenflächen dar.

Zur Entbuschung werden verschiedene Verfahren und Techniken mit unterschiedlichem Mechanisierungsgrad angewendet. Ihre Einsetzbarkeit auf schützenswerten Naturschutzflächen ist aufgrund naturschutzfachlicher und technisch/ technologischer Einschränkungen nicht immer gegeben, so dass sich insgesamt ein Anwendungsbereich in der Entbuschung darstellen lässt, welcher ein Defizit an naturschutzfachlich angepasster Technik zur Entbuschung aufweist.

Ziel dieser Arbeit ist es, den Handlungsspielraum des Naturschutzes und der Landschaftspflege durch die Entwicklung eines selbstschneidenden Häckslerkonzeptes zur vollmechanisierten Entbuschung von Offenflächenbiotopen in diesem Defizitbereich zu erweitern. Dabei soll neben den naturschutzfachlichen Belangen auch die Verwertbarkeit des Gehölzmaterials Beachtung finden.

Im Rahmen eines Entwicklungsprozesses kann in mehreren Stufen ein vollmechanisiertes Häckslerkonzept zur Entbuschung von Offenflächen erstellt werden, welches eine gleichzeitige Bergung des Gehölzmaterials ermöglicht.

Dazu wird anhand eines Vergleichs bestehender Verfahren der Anwendungsbereich der Entbuschung ermittelt. Unter Einbezug der dort vorliegenden naturschutzfachlichen sowie technologischen Anforderungen kann ein Basiskonzept für die Maschinenentwicklung geschaffen werden, auf dessen Grundlage drei zeitlich aufeinanderfolgende Prototypen eines schlepperbasierten Mähhäckslers erstellt werden.

Diese werden unter anspruchsvollen und vielseitig ausgeprägten Vegetationsbedingungen der Verbuschung getestet. Dabei werden Maschinenkonzepte und Komponenten auf Funktionsfähigkeit überprüft, bis letztlich ein funktionsfähiges Maschinenkonzept bereitgestellt wird. 


\section{Literaturverzeichnis}

Affenzeller, G.; Stampfer, K. (2007): Energieholzbereitstellung mit Traktor und Krananhänger mit Fallbeilklingenaggregat: Fallstudie im Forstbetrieb Fürst Esterhàzy'sche Privatstiftung Lockenhaus. Institut für Forsttechnik im Department für Wald- und Bodenwissenschaften, Universität für Bodenkultur Wien

AHWI Maschinenbau GmbH (2008): Aufnahmemulcher AM600:

Produktbeschreibung Erntesystem Biomasse: Entwicklung einer Maschine zum flächigen Bergen/ Aufsammeln von Biomasse

Bärisch, G.;Glaß, J.; Weigel, R. (1982): Grundlagen der Forsttechnik: Fachwissen des Forstingenieurs. Landwirtschaftsverlag, Berlin

Bauer, M.;Hartmann, K.;Meyer, K.;Nelles, M.;Reffelt, K.; Sowitzki, U. (2004): Handbuch Offenlandmanagement: Am Beispiel ehemaliger und in Nutzung befindlicher Truppenübungsplätze ; http://edok01.tib.uni-hannover.de/edoks/e01fb05/488127343.pdf

Bauer, M. (2005): Verbesserung der Qualität von Holzhackschnitzeln aus der Landschaftspflege für die energetische Verwendung: vorgelegt bei Arbeitsgemeinschaft industrieller Forschungsvereinigungen "Otto von Guericke" e.V. (AIF), Göttingen

Beinlich, B. (2002): Kalktrockenrasen, Kalkhalbtrockenrasen und nah verwandte Grünlandgesellschaften; Schriftenreihe EGGE-Weser 15 : 27- 38

Bertsch, B. (2008): Mündl. Auskunft. Landwirt, Göttingen- Roringen

Block, A.;Behn, W.;Lücke, W.; Denich, M. (2000): Buschhäckslereinsatz zur Sekundärwaldnutzung in tropischen Brachesystemen. Landtechnik 55 (3): 214-215

Block, A. (2004): Göttinger Mähhäcksler Tritucap und Forstmulcher- Nicht brennende Flächenvorbereitung am Beispiel der Zona Bragantina, Nord-Ost-Amazonien, Brasilien. Dissertation, Georg-AugustUniversität, Göttingen 
Böhmer, H.J.; Bender, O. (2000): Die Entwicklung der Wacholderheiden auf der nördlichen Frankenalb; In: Beiträge zur Landeskunde Oberfrankens; Bamberger Geografische Schriften, Sonderdruck (6) : 169- 189

Böhmer, H.J. (1994): Die Halbtrockenrasen der Fränkischen Alb; In: Mitteilungen der Fränk. Geografischen Gesellschaft 41 : 323- 343

Brenndörfer, M. (1995): Bodenverdichtung: FAL/KTBL-Fachtagung am 18./19. November 1993 in Braunschweig. Landwirtschaftsverl, Münster-Hiltrup

Brunk, I.;Beier, W.;Burkard, B.;Hinrichsen, A.;Oelschlaeger, S.;Prochnow, A.;Saure, C.;Vorwald, J.;Wallschläger, D.; Zierke, I. (2004): Beweidung mit Haustieren; In: Handbuch Offenlandmanagement, Springer, Berlin : 105- 120

Brunk, I.;Karlowski, U.; Wiegleb, G. (2001): Offenlandforschung auf Truppenübungsplätzen in Brandenburg. Tagungsband: Tagungsband "Renaturierungsökologie" der Gesellschaft für Ökologie (GfÖ), 21.23.Februar 2001 Burg (Spreewald)

Brusche, R. (1983): Hackschnitzel aus Schwachholz: Bergung, Lagerung und Trocknung; KTBL-Schrift 290. Landwirtschaftsverlag, MünsterHiltrup

Burger, F. (2004): Technologie und Ökonomie des Anbaus und der Ernte von Feldholz: Energieholzproduktion in der Landwirtschaft: Potential, Anbau, Technologie, Ökologie und Ökonomie ; Bornimer agrartechnische Berichte 35 35. ATB, Potsdam-Bornim

Burkart, B. (2006): Offenlandmanagement mit Haus- und Wildtieren: am Beispiel des ehemaligen Truppenübungsplatzes Dauban/Oberlausitz: Auswirkungen der Weide- und Äsungstätigkeit von Schafen, Ziegen und Elchen auf die Vegetation und deren Effektivität im Offenlandmanagement am Beispiel des ehemaligen Panzerschießplatzes Dauban in der Oberlausitz. Dissertation, AlbertLudwigs-Universität, Freiburg im Breisgau 
CEN/TS 14778-1 (2004): Europäisches Komitee für Normung: Feste Biobrennstoffe- Probennahme- Teil 1: Verfahren zur Probennahme, Brüssel

CEN/TS 14780 (2004): Europäisches Komitee für Normung: Feste Biobrennstoffe- Verfahren zur Probenherstellung, Brüssel

CEN/TS 14961 (2005): Europäisches Komitee für Normung: Feste Biobrennstoffe- Brennstoffspezifikationen und -klassen (Vornorm), Brüssel

CEN/TS 15149-1 (2005): Europäisches Komitee für Normung: Feste Biobrennstoffe- Verfahren zur Bestimmung der Teilchengrößenverteilung- Teil 1: Rüttelsiebverfahren mit Sieblochgrößen von 3,15mm und darüber, Brüssel

Cremer, T.;Becker, G.; Sauter, U. (2007): Mobilisierung und wirtschaftliche Nutzung von Rohholz aus Wald und Landschaft zur Energieerzeugung: Modellprojekt einer integrierten Bereitstellungs-, Logistik- und Verwertungskette für die Region Hochschwarzwald Breisgauer Bucht ; Abschlussbericht zu dem von der Deutschen Bundesstiftung Umwelt (DBU) geförderten Projekt. Univ., Inst. für Forstbenutzung und Forstliche Arbeitswissenschaft [u.a.], Freiburg

Cremer, T. (2008): Bereitstellung von Holzhackschnitzeln durch die Forstwirtschaft: Produktivitätsmodelle als Entscheidungsgrundlage über Verfahren und Aushaltungsvarianten, entwickelt auf der Basis einer Metaanalyse. Dissertation, Univ.Freiburg i. Br.

Denkinger, B. (2004): Wie forstliche Hacker arbeiten: Technische Merkmale und Bauarten. LWF aktuell 48: 21- 23

Deutscher Rat für Landespflege (1993): Truppenübungsplätze und Naturschutz: gutachtliche Stellungnahme und Ergebnisse eines Kolloquiums des Deutschen Rates für Landespflege; Schriftenreihe des Deutschen Rates für Landespflege 62, Bonn 
Deutscher Verband für Landschaftspflege e.V. (2008): BEST PRACTICE:

Erfolgsmodelle energetischer Nutzung von Biomasse aus der Landschaftspflege, Ansbach

Dillenburger, R. (2005): Mündl. Auskunft. Untere Naturschutzbehörde Göttingen am 21.02.2005, Göttingen

Engeln, W. (2011): Methoden der Produktentwicklung. OldenbourgIndustrieverlag, München

Falk, K.;Härdtle, W.; Mohamed, A. (2004): Auswirkungen des kontrollierten Brennens auf die pflanzenverfügbaren Nährstoffe in Podsol-Böden (NSG "Lüneburger Heide"); In: Feuer und Beweidung als Instrumente zur Erhaltung magerer Offenlandschaften in Nordwestdeutschland- Ökologische und sozioökonomische Grundlagen des Heidemamagements auf Sand- und Hochmoorstandorten; NNA-Berichte 17 (2), Schneverdingen

Felde, J.;Katz, C.; Blume, K. (1980): Handbuch Holzwirtschaft: Einsatz von Sägeblättern und Sägeblattbehandlung im Sägewerk. Dt. Betriebswirte-Verlag, Gernsbach

Felde, J. (2005): Schriftliche Mitteilung zur Auslegung von Sägeblättern im Anwendungsfall Gehölzmähhäcksler. Richard Felde GmbH \& Co.KG, Remscheid

Felinks, B. (2000): Primärsukzession von Phytozönosen in der Niederlausitzer Bergbaufolgelandschaft. Brandenburgische Techn. Univ., Dissertation, Cottbus, 2000

Feller, S.;Remler, N.; Weixler, H. (1998): Vollmechanisierte Waldhackschnitzel-Bereitstellung: Ergebnisse einer Arbeitsstudie am Hackschnitzel-Harvester, LWF, Freising

Firus, S.; Belter, A. (1995a): Bestimmung der Energien und Kräfte zum Zerkleinern ganzer Bäume im Prozeß der Rodung von Obstanlagen: Bericht zum Forschungsthema, Techn. Univ., Professur für Landmaschinen, Dresden 
Firus, S.; Belter, A. (1998): Energiesparende Zerkleinerung von Reisig, Rest- und Recyclingholz; In: Wissenschaftliche Zeitschrift der Technischen Universität Dresden 48 (2), Techn. Univ., Dresden : 96102

Firus, S.; Belter, A. (1995b): Kontinuierliches Aufnehmen und energiesparendes Zerkleinern von Restholz. Tagungsband: CIGR IV, 19.Conference, 25.- 29.9.1995 in Hohenheim, Hohenheim

Gabriel, O. (2003): Mit Rückezug und Fällgreifer in die Durchforstung. Forst \& Technik (10): 4- 6

Geyer \& Dolek (2011): Gefährdung des Violetten Feuerfalters ; http://www.geyer-und-dolek.de (20.02.2011)

Googlemaps (2011): Kartenmaterial erstellt mit Googlemaps ; http://maps.google.de/

Grimm, R. (1980): Möglichkeiten zur Mechanisierung der Knickpflege. Dissertation, Universität Kiel

Grote, K.;Feldhusen, J.; Dubbel, H. (2007): Dubbel: Taschenbuch für den Maschinenbau. Springer, Berlin ;Heidelberg

Grothaus, H.P. (1993): Nachwachsender Rohstoff: Energiewald- Nutzung von schnellwachsenden Hölzern zur Rohstoff- und Energiegewinnung. Diplomarbeit, Georg- August- Universität, Göttingen

Hakkila, P. (1989): Utilization of residual forest biomass. Springer, Berlin

Hartmann, H.; Thuneke, K. (1997): Ernteverfahren für Kurzumtriebsplantagen: Maschinenerprobung und Modellbetrachtungen. Inst. u. Bayer. Landesanstalt für Landtechnik d. Techn. Univ. München-Weihenstephan, Freising

Haschke, P. (2000): Beitrag zur Standardisierung von Holzbrennstoffen. Fachhochschule Eberswalde, Fördergesellschaft erneuerbare Energien e.V, Eberswalde

Heike, A. (2008): Mündl. Auskunft. Bröcker GmbH, Wendeburg 


\section{Institut für Agrartechnik Bornim ; VDI- Gesellschaft für Agrartechnik}

(1994): Technik und Verfahren zur Landschaftspflege und für die Verwertung der anfallenden Materialien: Kolloquium in PotsdamBornim am 20. und 21. Januar 1994; Bornimer agrartechnische Berichte 4. Wissenschaftlicher Fachverl. Fleck, Niederkleen

Jedicke, E. (1996): Praktische Landschaftspflege: Grundlagen und Maßnahmen. Ulmer, Stuttgart (Hohenheim)

Jones, D.H. (1997): A Trial of Two Small Scale Chippers. Technical Development Branch, Forestry Commission Glantwymyn

Kaltschmitt, M. (2001): Energie aus Biomasse: Grundlagen, Techniken und Verfahren. Springer, Berlin

Kesting, S.; Isselstein, J. (2006): Beitrag des Departments für Graslandwissenschaften: 2. Zwischenbericht zum DBU- Projekt "Vollmechanisierte Landschaftspflege in Naturschutz- und FFHGebieten", Göttingen

Kollmann, J.; Staub, F. (1995): Entwicklung von Magerrasen am Kaiserstuhl nach Entbuschung. Ökologie und Naturschutz 4: 87- 103

Kromer, K.; Reloe, H. (1991): Technik der Landschaftspflege. AID, Bonn

Küppers, J. (1997): Betriebswirtschaftliche und erntetechnische Begleitforschung zum Anbau schnellwachsender Baumarten auf landwirtschaftlichen Flächen. Arbeitsbericht des Instituts für Ökonomie (2): 83- 87

Leinonen, A. (2007): Wood chip production technology and costs for fuel in Namibia. VTT Tiedotteita, Espoo

Levin, M. (2004): Mündl. Mitteilung. Stadtforstamt Göttingen, Göttingen

Lindemann, U. (2005): Methodische Entwicklung technischer Produkte: Methoden flexibel und situationsgerecht anwenden. Springer-Verlag Berlin Heidelberg, Berlin, Heidelberg

Löbbert, M. (2001): Landschaftspflege. Landtechnik 56 (1): 234- 236 
LWK- Hannover (2006): Hohe Holznachfrage in Niedersachsen ;

http://www.lwkhannover.de/index.cfm/portal/forstwirtschaft/nav/0/article/6985.html

Maier, G. (1983): Spanungstechnische Grundlagen der Hackschnitzelerzeugung in Trommelhackern; In: Holz als Roh- und Werkstoff 41 : 437- 439

Mann, S.; Böhmer, J. (2008): GIS-gestützte Analyse von holzartigen Biomassepotenzialen aus der Landschaftspflege und deren Eignung für die energetische Verwertung - ein Beispiel aus dem Unteren Saaletal. Biomasse-Tagung am Umweltcampus Birkenfeld vom 06.11. bis 07.11.2008;

Martin, H.;Römisch, P.; Weidlich, A. (2008): Materialflusstechnik: Auswahl und Berechnung von Elementen und Baugruppen der Fördertechnik. Vieweg, Wiesbaden

Meinecke, T. (2005): Mündl. Mitteilung. Umweltbiologische Studien- Dr. Meinecke, Ebergötzen

Meineke, T. (2006): Auswirkungen eines neuartigen Gehölzmähhäckslers auf die Vegetation und Fauna von Sukzessionsflächen im FFHGebiet "Göttinger Wald": 2. Zwischenbericht zum DBU- Projekt "Vollmechanisierte Landschaftspflege in Naturschutz- und FFHGebieten", Göttingen

Meyer-Ludorf (2009): Webseite ; http://www.naturdurchtechnik.de/ (10.02.2009)

Müller, J.; Schaltegger, S. (2004): Sozioökonomische Analyse des Heidemanagements in Nordwestdeutschland: Wirtschaftlichkeit, Kosten- Wirksamkeitsverhältnisse und Akzeptanz; In: Feuer und Beweidung als Instrumente zur Erhaltung magerer Offenlandschaften in Nordwestdeutschland- Ökologische und sozioökonomische Grundlagen des Heidemamagements auf Sand- und Hochmoorstandorten; NNA-Berichte 17 (2), Schneverdingen : 183197 
Münzel, M.; Schumacher, W. (1993): Magerrasen schützen; AidSchriftenreihe 2503. KDV, Lengerich

MULAG Fahrzeugwerk Heinz Wössner (2006): MULAG

Heckenschneidkopf HSK 1200: Produktvorstellung MULAG Fahrzeugwerk Heinz Wössner GmbH u. Co. KG

Naturstiftung David (2010): Energieholz und Biodiversität - Die Nutzung von Energieholz als Ansatz zur Erhaltung und Entwicklung national bedeutsamer Lebensräume: Zwischenbericht (Berichtszeitraum 01.07.2009 - 31.03.2010) zum Projekt "Energieholz und Biodiversivität", Erfurt

Nurmi, J. (1986): Chunking and chipping with conescrew chipper; Folia forestalia 659 , Helsinki

Peringer, A. (2008): Analyse von Mechanismen der sekundären progressiven Sukzession von voralpinem Niedermoorgrünland zu Erlenbruchwald. Univ., Diss--Stuttgart, 2008

Persson, S. (1987): Mechanics of cutting plant material. American Society of Agricultural Engineers, St. Joseph, Michigan

Ponn, J.; Lindemann, U. (2008): Konzeptentwicklung und Gestaltung technischer Produkte: Optimierte Produkte - systematisch von Anforderungen zu Konzepten. Springer, Berlin ;Heidelberg

Prochnow, A.; Schlauderer, R. (2002): Verfahren der Landnutzung zur Offenhaltung ehemaliger Truppenübungsplätze. Landtechnik 57 (3): 150-151

Rabeler, J. (2008): Fa. mera-Rabeler. mündl. Auskunft, Göttingen

Rahmann, G. (2000): Biotoppflege als neue Funktion und Leistung der Tierhaltung: dargestellt am Beispiel der Entbuschung von Kalkmagerrasen durch Ziegenbeweidung. Kovac, Hamburg 
Raths, U.;Riecken, U.; Ssymank, A. (1995): Gefährdung von Lebensraumtypen in Deutschland und ihre Ursachen: Auswertung der Roten Liste gefährdeter Biotoptypen. Natur und Landschaft 70 (5): 203- 212

Rotter, D. (2006): Einfluss der Verbuschung auf Artengemeinschaften auf Heißländen in der Unteren Lobau; Wissenschaftliche Reihe Nationalpark Donau-Auen 22

Sauter, J.;Krawutschke, M.; Noto, F. (2008): Bis zu welcher Hangneigung kann ein Traktor mähen? Tagungsband: Agricultural Engineering LAND.TECHNIK 2008: Tagung Stuttgart-Hohenheim, 25.-26. September 2008, VDI Verlag, Düsseldorf

Schlecht, B. (2010): Getriebe - Verzahnungen - Lagerungen. Pearson Studium, München [u.a.]

Schmidt, G. (2006): Mündl. Auskunft. Schmidt Maschinenbau GmbH, Uchte Schmidt, G. (2008): Mündl. Auskunft. Schmidt Maschinenbau GmbH, Uchte Scholz, V.; Idler, C. (2005): Energieverlust und Schimmelpilzentwicklung bei der Lagerung von Feldholz-Hackgut; Bornimer agrartechnische Berichte 39 39. ATB, Potsdam-Bornim

Scholz, V.;Idler, C.; Egert, J. (2006): Probleme bei der Hackschnitzellagerung: Untersuchungen zu Schimmelpilzentwicklung und Energieverlusten bei der Lagerung von Feldholzhackschnitzeln. Holz- Zentralblatt 7 (27): 804- 806

Scholz, V.;Idler, C.;Daries, W.; Egert, J. (2005): Lagerung von Feldholzhackgut. Landtechnik 60 (4): 210- 211

Scholz, V. (2007): Mechanisierungslösungen für die Ernte von schnellwachsenden Baumarten. Energie Pflanzen (1): 8- 10 
Schreiber, K.;Broll, G.; Brauckmann, H. (2000): Methoden der Landschaftspflege: eine Bilanz der Bracheversuche in BadenWürttemberg ; http://www.kalkmagerrasen.de/naturschutz/themen/bracheversuche. pdf

Stübig, D.K. (2000): Verfahren zur Nutzung von Knick- und Schwachholz für dezentrale Heizwerke. Univ., Diss.--Kiel, 2000

Tamm, J. (2008): Leitlinien der Maßnahmenplanung in MagerrasenLebensraumtypen: Vortrag im Rahmen der Veranstaltung der Naturschutz-Akademie Hessen (NAH) „Maßnahmen in Natura 2000Gebieten - Lebensraumtyp Magerrasen“ am 11. Juni 2008 in Wetzlar

Tscheuschner, H. (2004): Grundzüge der Lebensmitteltechnik. Behr, Hamburg

Universität Kassel (2005): Zucht einer Landschaftspflegeziege ; http://www.uni-kassel.de/fb11/ntier/lpz.html

von Drachenfels, O. (2004): Kartierschlüssel für Biotoptypen inNiedersachsen unter besonderer Berücksichtigung der nach § $28 \mathrm{a}$ und § 28b NNatG geschützten Biotope sowie der Lebensraumtypen von Anhang I der FFH-Richtlinie, Stand März 2004; In: Naturschutz Landschaftspfl. Niedersachs. A/4 (1), Hildesheim

Wanner, M.;Anders, K.;Brunk, I.;Burkard, B.;von Dorsten, P.;Fürstenau, S.;Oelschlaeger, S.;Prochnow, A.;Wiesener, C.; Xylander, W.E. (2004): Offenhaltung durch Feuer; In: Handbuch Offenlandmanagement, Springer, Berlin : 153- 168

Wasner, U. (1987): Wirkung der Saugmahd auf den Insektenbestand am Straßenrand; In: LÖLF- Mitteilungen (2) : 34- 39

Wiegmann, K. (2007): Bioenergie und Naturschutz: Sind Synergien durch die Energienutzung von Landschaftspflegeresten möglich?: Endbericht an das Bundesministerium für Umwelt, Naturschutz und Reaktorsicherheit, Darmstadt [u.a.] 
Wieneke, F.; Döhrer, K. (1994): Mähhacker für Gehölze. Offenlegungsschrift DE 4238007 A1, Deutsches Patentamt

Wieneke, F. (1957): Untersuchung zur Erklärung und Beseitigung von Wickelerscheinungen an umlaufenden Maschinenteilen. Landtechnische Forschung 7 (1): 1 - 8

Wieneke, F. (1993): Mähhacker für Energieplantagen von Pappeln und Weiden. Landtechnik 48 (12): 646- 647

Wilhelm, K. (2004): Mündl. Mitteilung. Werkstatt der Abteilung Agrartechnik, Göttingen

Wittkopf, S.;Hömer, U.; Feller, S. (2003): Bereitstellungsverfahren für Waldhackschnitzel: Leistungen, Kosten, Rahmenbedingungen. Bayer. Landesanst. für Wald $u$. Forstwirtschaft, Freising

Wittkopf, S. (2005): Bereitstellung von Hackgut zur thermischen Verwertung durch Forstbetriebe in Bayern. Dissertation, Techn. Universität, München 


\section{Danksagung}

In diesem Teil dieser Arbeit soll allen, die weder in Verzeichnissen oder Nennungen dieses Manuskriptes erwähnt werden, aber zum Entstehen dieser Arbeit beigetragen oder sie auch erst ermöglicht haben, gedankt werden. Ihre Unterstützung wird mir immer in Erinnerung bleiben.

Herrn Prof. Dr. Lücke möchte ich meinen Dank dafür aussprechen, dass er mich damals mit dem Thema der Arbeit betraut hat und mich dem Themenkomplex "vollmechanisierte Entbuschung“ mit recht freier Hand widmen ließ. Sein Vertrauen in mich und sein Engagement bei allen schwierigen Projektsituationen hat mir einen großen Rückhalt gegeben.

Für die vielen thematischen und inspirierenden Diskussionen, Hilfestellungen und letztlich auch einer Korrekturlesung dieser Arbeit möchte ich mich bei Herrn Dr. Block ganz besonders bedanken. Seine Erfahrungen und seine konstruktiven Anregungen haben zum Gelingen dieser Arbeit beigetragen. Der Dank über die herzliche Aufnahme bei so einigen Übernachtung während der Projektphase gilt seiner gesamten Familie.

Unserer Werkstattbesetzung Herrn "Charly“ Marten, Herrn Karsten Wilhelm, Herrn „Siggi“ Opolka und Herrn „Harry“ Krumrey möchte ich meinen besten Dank aussprechen. Ihre konstruktive Kritik, ihre Verwirklichung meiner „fleischlosen“ Ideen zu „stahlharten“ Resultaten und ihr Einsatz „über den Feierabend hinaus" war bei der Durchführung des Projektes unabdingbar.

Herrn Gerhard Schmidt gilt mein Dank für die vielen maschinenbaulichen Ideen und Lösungen sowie das Unmögliche möglich zu machen. Sein Tatendrang, sein Elan und letztlich auch der freundschaftliche Kontakt zu ihm und seiner Familie wird mir in Erinnerung bleiben. Besonderer Dank gilt dabei auch seiner Tochter Angela, die uns umfangreich mit technischen Zeichnungen versorgt hat.

Horst Schmidt möchte ich meinen Dank aussprechen für das großzügige Vertrauen, mit dem er mich mit den Schleppern Schalten und Walten ließ. Seine Anregungen aus der Praxis waren mehr als einmal hilfreich. In diesem Zusammenhang möchte ich mich auch bei allen weiteren Projektpartnern 
bedanken, die an der Lösung dieser Aufgabe mitgewirkt haben: Herrn Dr. Meineke, Herrn Prof. Isselstein, Herrn Kesting, Herrn Levin, Herrn Dillenburger und Frau Tippach-Kemmling,

Nicht vergessen werden dürfen natürlich die „Hände“ im Hintergrund: Frau Weitemeyer für die vielseitigen verwaltungstechnischen Belange und Frau Grube für das Auffinden jeglicher verschollener Literatur.

Der größte Dank gilt meiner Familie, die mich bei dieser Arbeit entbehrt und unterstützt hat. Meine Frau Alexandra und meine Kinder Carlotta und Greta haben mir besonders viel Kraft dazu gegeben. Ich bin sehr glücklich, dass ich die drei habe.

Bei meinen Eltern möchte ich mich herzlich bedanken, dass sie mir diesen Weg ermöglicht haben und mir dabei immer zur Seite standen.

Ganz besonderer Dank gilt zudem meinem Bruder Helge für die abendfüllenden Diskussionen über die Häckseltechnik sowie auch für die Unterstützung und die Teilnahme am Bau unseres eigenen Schneckenhackers, der mir einen erheblichen Erkenntnisgewinn gebracht hat. Er funktioniert einfach bestens!

Danke!

Das Projekt wurde von der Deutschen Bundesstiftung Umwelt (DBU) finanziell gefördert. Ich möchte der DBU auch im Namen aller Beteiligten meinen Dank aussprechen, dass dieses Projekt ermöglicht wurde. 


\section{Lebenslauf}

\section{Persönliche Daten}

Geburtsdatum

30.07.1977

Geburtsort

Tulln/ Niederösterreich

Familienstand

verheiratet, zwei Kinder

\section{Berufliche Stationen}

03/2010 bis heute

selbständiger Energieholzproduzent/ -händler

10/2009 bis 02/2010 Vorbereitung der Selbständigkeit

03/2009 bis 09/2009 Entwicklungsingenieur NawaRo in der

Entwicklungsabteilung eines Landtechnikherstellers

03/2005 bis 02/2009 Wissenschaftlicher Mitarbeiter in der Abteilung Agrartechnik des Departments für Nutzpflanzenwissenschaften der Georg- AugustUniversität Göttingen

10/2004 bis 02/2005 Wissenschaftliche Hilfskraft in der Abteilung Agrartechnik des Departments für Nutzpflanzenwissenschaften der Georg- AugustUniversität Göttingen

\section{Studium}

10/1998 bis 03/2004 Studium der Agrarwissenschaften in der Fachrichtung Pflanzenproduktion mit den Schwerpunkten Regenerative Energien sowie Pflanzenbau und Pflanzenernährung

- Abschluss: Master of Science

- Masterarbeit mit dem Thema: „Mikrowellentrocknung von PESUFilterkerzen unter besonderer Berücksichtigung der Filterintegrität"

Schulbildung und Wehrdienst

09/1997 bis 06/1998

08/1988 bis 06/1997
Grundwehrdienst im Panzerartilleriebataillon 15 in Stadtoldendorf;

Jugenddorf Christophorus-Schule Elze mit Abschluss der Hochschulreife 